\title{
ASYMPTOTICALLY EFFICIENT ESTIMATORS FOR \\ GEOMETRIC SHAPE FITTING AND \\ SOURCE LOCALIZATION
}

\author{
A Thesis \\ presented to \\ the Faculty of Graduate School \\ University of Missouri
}

In Partial Fulfillment

of the Requirements for the Degree

of Doctor of Philosophy

by

ZHENHUA MA

Dr. Dominic K.C. Ho, Thesis Supervisor

DECEMBER 2013 
The undersigned, appointed by the Dean of the Graduate School, have examined the thesis entitled:

\section{ASYMPTOTICALLY EFFICIENT ESTIMATORS FOR GEOMETRIC SHAPE FITTING AND SOURCE LOCALIZATION}

presented by Zhenhua Ma

a candidate for the degree of Doctor of Philosophy

and hereby certify that, in their opinion, it is worthy of acceptance.

Dr. Dominic K.C. Ho

Dr. Justin Legarsky

Dr. Yi Shang

Dr. Zhihai He 
I would like to express my sincere thankfulness to my father, Gengli Ma, my mother, Manna Ling, my wife, Mei Yang and all my other family members. Without your endless love, support and encouragement, I would not be able to accomplish my goal. Thank you for always being there for me!

I also want to say thank you to all my dear friends, especially Le Yang, Liyang Rui, Fei and Jeremy. Thanks for the helps from all of you. I really enjoy the time being with all of you! 


\section{ACKNOWLEDGEMENTS}

I would like to take this opportunity to gratefully acknowledge everyone who made this thesis possible. First I want to express my sincere appreciation to my supervisor, Dr. Dominic K.C. Ho, for his continued patience, support, inspiration and invaluable guidance in my academic research and in many other aspects of my life. I learned a lot from him on being a cautious and careful researcher, managing time efficiently, being an organized and responsible person. Without his great help and guidance, I would not be able to accomplish this thesis.

I would also like to express my sincere gratitude to my committee members Dr. Justin Legarsky, Dr. Yi Shang and Dr. Zhihai He for spending their valuable time and giving important suggestions that improved the quality of this thesis.

Last but not least, I would like to thank University of Missouri, for giving me the opportunity to pursue my Doctor of Philosophy degree, receive high quality education and obtain wonderful life experience that enhance my personality and made me a better man. 


\section{TABLE OF CONTENTS}

ACKNOWLEDGEMENTS................................................................................... ii

LIST OF TABLES......................................................................... viii

LIST OF FIGURES............................................................................... ix

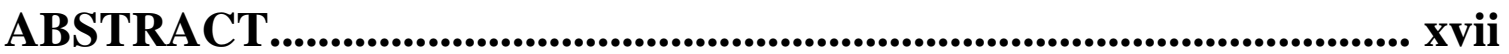

\section{Chapter 1}

Introduction $\quad 1$

1.1 Research Areas and Motivations.......................................................................................... 1

1.1.1 Circle and Ellipse Fitting................................................................................................... 2

1.1.2 Source Localization........................................................................................................... 4

1.2 Circle and Ellipse Fittings Basics....................................................................................... 8

1.2.1 Literature Survey...................................................................................................... 8

1.2.2 Semi-definite Programming and Relaxation for Circle Fitting........................... 10

1.3 Source Localization Basics................................................................................................ 12

1.3.1 Source Localization Measurements....................................................................... 12

1.3.2 Literature Survey of Source Localization Methods.............................................. 16

1.3.3 Overview of TOA Source Localization Methods.................................................. 17

1.4 Organization of the Thesis................................................................................................. 18

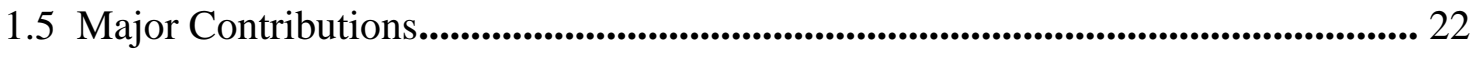




\section{Chapter 2}

Maximum Likelihood and Full Least-squares Estimators for Circle 25

2.1 The Circle Fitting Problem...................................................................................................... 25

2.2 The ML Solution......................................................................................................................... 26

2.3 The FLS Solution................................................................................................................... 28

2.4 Comparison........................................................................................................................... 29

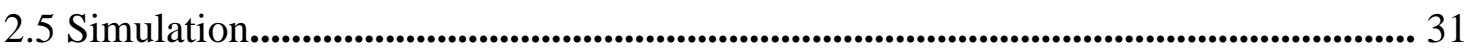

2.6 Concluding Remarks.......................................................................................................... 32

\section{Chapter 3}

Circle Fitting Using Semi-definite Programming 36

3.1 Problem Formulation.................................................................................................... 36

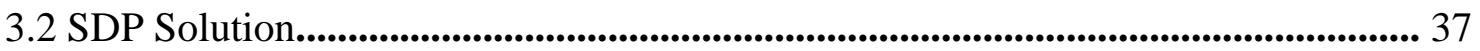

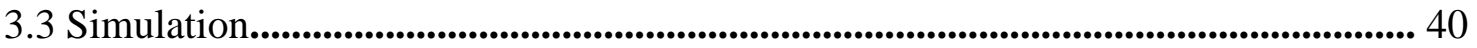

3.4 Concluding Remarks.................................................................................................................... 42

\section{Chapter 4}

Asymptotically Efficient Estimators for the Fittings of Coupled Circles $\begin{array}{ll}\text { and Ellipses } & 46\end{array}$

4.1 Problem Formulation................................................................................................................ 46

4.2 Proposed Fitting Solutions......................................................................................................... 48 


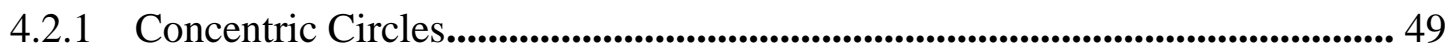

4.2.2 Concentric Ellipses................................................................................................... 52

4.3 KCR Lower Bound....................................................................................................................... 56

4.3.1 KCR Lower Bound for Concentric Circles Fitting.............................................. 57

4.3.2 KCR Lower Bound for Concentric Ellipses Fitting.............................................. 58

4.4 Performance Analysis............................................................................................. 59

4.4.1 Concentric Circles............................................................................................................ 59

4.4.2 Concentric Ellipses........................................................................................................ 60

4.5 Simulation and Experiment................................................................................................... 64

4.5.1 Synthetic Data.................................................................................................................... 64

4.5.2 Experiment with Real Images............................................................................. 69

4.6 Concluding Remarks..................................................................................................................... 73

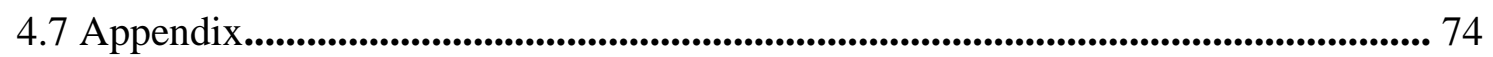

\section{Chapter 5}

\section{TOA Localization in the Presence of Random Sensor Position Errors 88}

5.1 TOA Localization Problem and Its CRLB and MSE Analysis.................................... 88

5.2 Proposed Solution........................................................................................................................... 92

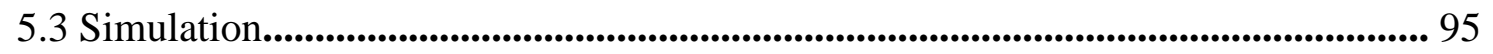

5.4 Concluding Remarks............................................................................................................. 96 


\section{Chapter 6}

\section{Joint Source Localization and Sensor Position Refinement for Sensor}

Networks

6.1 Problem Formulation and Proposed Solution. 99

6.2 Performance Analysis............................................................................................................. 105

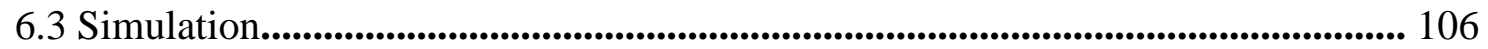

6.4 Concluding Remarks................................................................................................................... 109

\section{Chapter 7}

\section{A Study on the Effects of Sensor Position Error and Calibration}

\section{Emitter Placement for Source}

7.1 Localization Problem.............................................................................................................. 112

7.2 Performance when Ignoring Sensor Position Errors .................................................... 115

7.3 CRLB and MSE Comparison.................................................................................................. 117

7.3.1 Conditions for Identical CRLB and MSE............................................................ 117

7.3.2 Increase in CRLB due to Sensor Position Errors................................................ 119

7.4 Geometry Independent Condition for Different Positiontings................................... 120

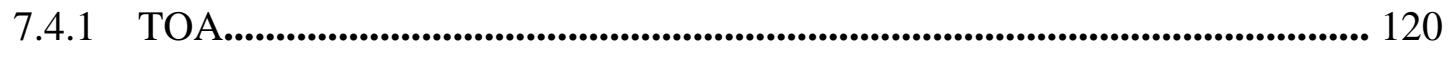

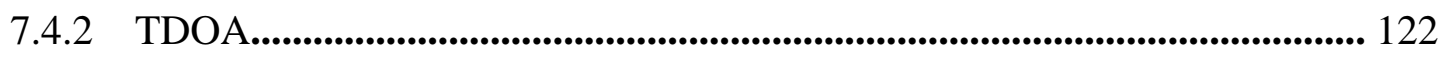

7.4.3 AOA...................................................................................................................... 123

7.5 Optimum Calibration Position.............................................................................................. 125 


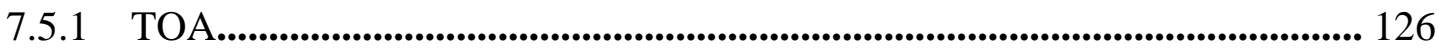

7.5.2 TDOA

7.5.3 AOA...................................................................................................................... 130

7.5.4 Suboptimum Calibration Emitter Position............................................................ 130

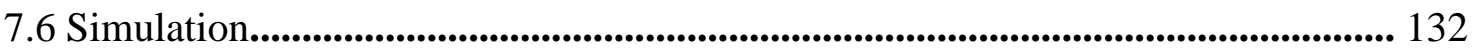

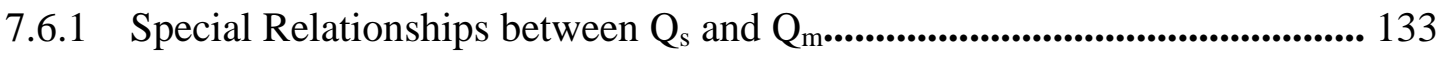

7.6.2 Placement of Calibration Emitter........................................................................ 134

7.7 Concluding Remarks.................................................................................. 137

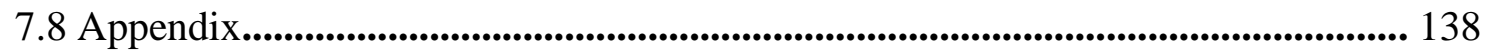

7.8.1 A: Evaluation of $\operatorname{FIM}\left(\mathbf{u}^{0}\right)$ in the presence of a Calibration Emitter............... 138

7.8.2 B: Maximizing (7.80) in the PD Sense under Special Cases............................ 142

\section{Chapter 8}

Summary and Future Work............................................................................. 150

8.1 Research Summary...................................................................................................................... 150

8.2 Future Research Work............................................................................................................. 155

$\begin{array}{ll}\text { Bibliography } & 157\end{array}$ 


\section{LIST OF TABLES}

Table

4.1 Bias results (in $\mathrm{dB}$ ) from the proposed concentric circles and concentric ellipses estimators with different weighting matrices......................................................................... 75

4.2 Computation time of the non-iterative and iterative solutions of the proposed estimators, the O'Leary method, the Kasa method for concentric circles and concentric ellipses fittings.................................................................................................... 76

4.3 Averaged performance difference (in $\mathrm{dB}$ ) with the KCR lower bounds of different

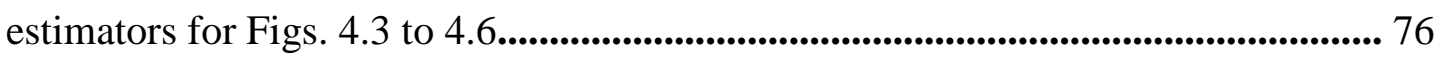

4.4 Comparisons of the total-square errors between the 50 data points and their closest points on the fitted ellipses from the proposed method and the O'Leary method....... 76

5.1 True Sensor Positions............................................................................................................................. 96

7.1 Averaged computation time of the proposed J criterion and the corresponding CRLB criterion of the source location estimate............................................................................... 144 


\section{LIST OF FIGURES}

$\begin{array}{ll}\text { Figure } & \text { Page }\end{array}$

1.1 Source localization using TOA measurement................................................................... 13

1.2 Source localization using AOA measurement........................................................................ 14

1.3 Source localization using TDOA measurement.................................................................... 15

2.1 Performance comparison of the ML and FLS method when $N=5, r=10$ and $\beta=$

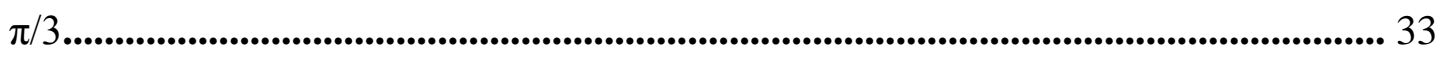

2.2 Performance comparison of the ML and FLS method when $N=5, r=10$ and $\beta=$

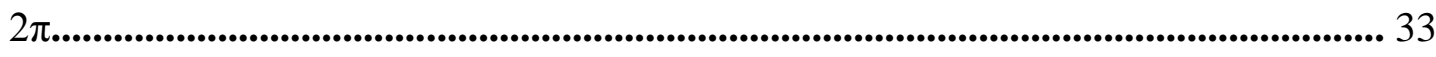

2.3 Averaged fitted circles of the ML and FLS method when $N=5, r=10$ and $\beta=2 \pi$ and

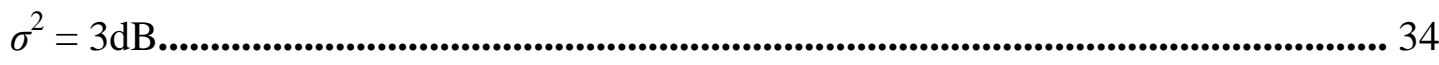

2.4 Performance comparison of the ML and FLS method when $N=20, r=10$ and $\beta=$

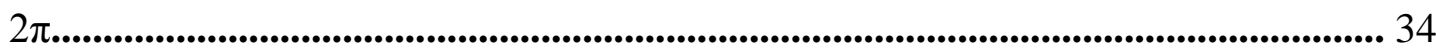

2.5 Performance comparison of the ML and FLS method when $N=20, r=2$ and $\beta=$

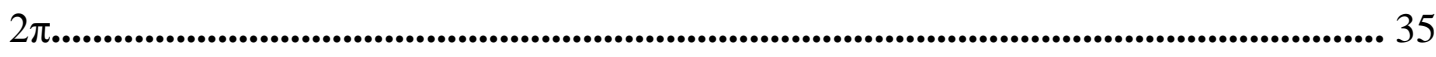

3.1 Distribution of the true data points on the circle, $M=5$ and $\beta=2 \pi$............................. 43

3.2 Performance comparison of the SDP and the Kasa methods when $M=5$ and $\beta=2 \pi$.

The upper set of results is for circle center and the lower set is for circle radius........ 43

3.3 Fitted circle from the SDP solution when $M=5$ and $\beta=2 \pi$, the noise power is $2 \ldots . .44$ 
3.4 Performance comparison of the SDP and the Kasa methods when $M=5$ and $\beta=\pi$. The upper set of results is for circle center and the lower set is for circle radius........ 44

3.5 Performance comparison of the SDP and the Kasa methods when $M=5$ and $\beta=\pi / 3$. The results are for the mses of circle center and radius together since the mse of circle center and the mse of circle radius are nearly the same....................................................... 45

3.6 Performance comparison of the SDP and the Kasa methods when $M$ varies, $\beta=2 \pi$ and the noise power is equal to 1 . The upper set of results is for circle center and the lower set is for circle radius........................................................................................... 45

4.1 Comparison of the proposed estimator, the O'Leary method and the Kasa method with the KCR lower bound for the estimations of the concentric circles center $\mathbf{c}^{\mathrm{o}}=\left[\begin{array}{ll}0 & 0\end{array}\right]^{\mathrm{T}}$ with data points from the two arc segments between 0 and $\pi$ rad when the noise

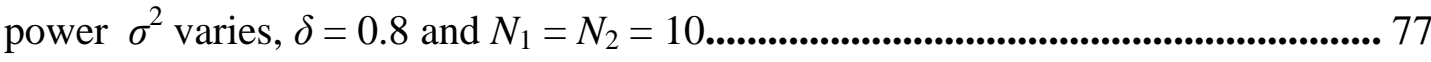

4.2 Comparison of the proposed estimator, the O'Leary method and the Kasa method with the KCR lower bound for the estimations of the concentric circles radii $r_{l}{ }^{\circ}=10$ and $r_{2}^{\mathrm{o}}=20$ with data points from the two arc segments between 0 and $\pi \mathrm{rad}$ when the

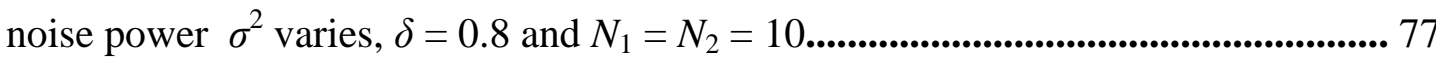

4.3 Comparison of the non-iterative solution from the proposed estimator, the O'Leary method and the Kasa method with the KCR lower bound for the estimations of the concentric circles center $\mathbf{c}^{\mathrm{o}}=\left[\begin{array}{ll}0 & 0\end{array}\right]^{\mathrm{T}}$ with data points from the two arc segments between 0 and $\pi \mathrm{rad}$ when the noise power $\sigma^{2}$ varies, $\delta=0.8$ and $N_{1}=N_{2}=10 \ldots \ldots . .78$ 
4.4 Comparison of the non-iterative solution from the proposed estimator, the O'Leary method and the Kasa method with the KCR lower bound for the estimations of the concentric circles radii $r_{1}{ }^{\circ}=10$ and $r_{2}{ }^{\circ}=20$ with data points from the two arc segments between 0 and $\pi$ rad when the noise power $\sigma^{2}$ varies, $\delta=0.8$ and $N_{1}=N_{2}=$

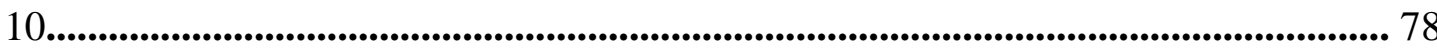

4.5 Comparison of the proposed estimator, the Kasa method and the Pratt method with the KCR lower bound for the estimations of the single circle center $\mathbf{c}^{\mathrm{o}}=\left[\begin{array}{ll}0 & 0\end{array}\right]^{\mathrm{T}}$ when

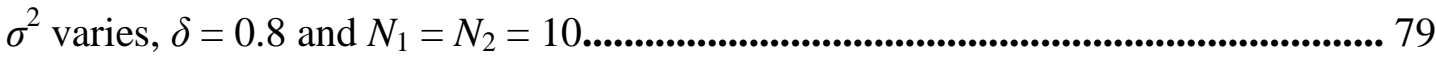

4.6 Comparison of the proposed estimator, the Kasa method and the Pratt method with the KCR lower bound for the estimations of the single circle radius $r_{l}{ }^{\circ}=10$ when $\sigma^{2}$

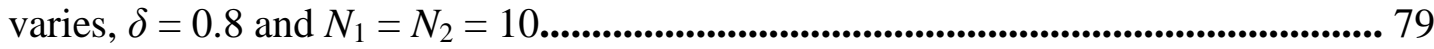

4.7 Comparison of the proposed estimator, the FNS method and the HEIV method with the KCR lower bound for the estimations of the concentric ellipses center $\mathbf{c}^{\mathrm{o}}=\left[\begin{array}{ll}0 & 0\end{array}\right]^{\mathrm{T}}$ when $\sigma^{2}$ varies from $10^{-4}$ to $1, N_{1}=N_{2}=10$ and $\delta=-0.6$. The data points are sampled from the concentric ellipses portions that are above the common major axis............. 80 4.8 Comparison of the proposed estimator, the FNS method and the HEIV method with the KCR lower bound for the estimations of $\varepsilon^{\circ}=0.25$ (upper) and $\Gamma_{1}{ }^{\circ}\left(\mathrm{A}_{1}{ }^{\mathrm{o}}=0.0075\right.$, $\mathrm{B}_{1}{ }^{\mathrm{o}}=0.0175, \rho_{1}{ }^{\mathrm{o}}=-0.0087$ ) (lower) when $\sigma^{2}$ varies from $10^{-4}$ to $1, N_{1}=N_{2}=10$ and $\delta$ $=-0.6$. The data points are sampled from the concentric ellipses portions that are above the common major axis................................................................................ 80 
4.9 Comparison of the non-iterative solution of the proposed estimator, the O'Leary method and the Taubin method with the KCR lower bound for the estimations of the concentric ellipses center $\mathbf{c}^{\mathrm{o}}=\left[\begin{array}{ll}0 & 0\end{array}\right]^{\mathrm{T}}$ when $\sigma^{2}$ varies from $10^{-4}$ to $1, N_{1}=N_{2}=10$ and $\delta$ $=-0.6$

4.10 Comparison of the non-iterative solution of the proposed estimator, the O'Leary method and the Taubin method with the KCR lower bound for the estimations of $\varepsilon^{\mathrm{o}}$ $=0.25$ (upper) and $\Gamma_{1}{ }^{\mathrm{o}}\left(\mathrm{A}_{1}{ }^{\mathrm{o}}=0.0075, \mathrm{~B}_{1}{ }^{\mathrm{o}}=0.0175, \rho_{1}{ }^{\mathrm{o}}=-0.0087\right)$ (lower) when $\sigma^{2}$ varies from $10^{-4}$ to $1, N_{1}=N_{2}=10$ and $\delta=-0.6 \ldots \ldots \ldots \ldots \ldots \ldots \ldots \ldots \ldots \ldots \ldots \ldots \ldots \ldots \ldots \ldots \ldots \ldots . \ldots \ldots 1$

4.11 Comparison of the iterative solution of the proposed estimator, the Taubin method and the Fitzgibbon method with the KCR lower bound for the estimations of the single ellipse center $\mathbf{c}^{\mathrm{o}}=\left[\begin{array}{ll}0 & 0\end{array}\right]^{\mathrm{T}}$ when $\sigma^{2}$ varies from $10^{-4}$ to $1, N_{1}=N_{2}=10$ and $\delta=-$ 0.6. The data points are sampled from the single ellipse portion that is above the major axis.

4.12 Comparison of the iterative solution of the proposed estimator, the Taubin method and the Fitzgibbon method with the KCR lower bound for the estimations of $\boldsymbol{\Gamma}_{1}{ }^{\mathrm{o}}$ $\left(\mathrm{A}_{1}{ }^{\mathrm{o}}=0.0075, \mathrm{~B}_{1}{ }^{\mathrm{o}}=0.0175, \rho_{1}{ }^{\mathrm{o}}=-0.0087\right)$ when $\sigma^{2}$ varies from $10^{-4}$ to $1, N_{1}=10$ and $\delta=-0.6$. The data points are sampled from the single ellipse portion that is above the major axis.................................................................................................... 82

4.13 Original image of concentric circles with zero-mean Gaussian white noise added (left) and the corresponding image after edge detection (right) 
4.14 Comparison of the proposed estimator and the O'Leary method for the variance of the concentric circles center estimate when the noise power $\xi^{2}$ varies and $N_{1}=N_{2}=$ 20.

4.15 Comparison of the proposed estimator and the O'Leary method for the variance of the concentric circles radii estimate when the noise power $\xi^{2}$ varies and $N_{1}=N_{2}=$ 20

4.16 Original image of concentric ellipses with zero-mean Gaussian white noise added (left) and the corresponding image after edge detection (right)

4.17 Comparison of the proposed estimator and the O'Leary method for the variance of the concentric ellipses center estimate when the noise power $\xi^{2}$ varies and $N_{1}=N_{2}=$ 20 85

4.18 Comparison of the proposed estimator and the O'Leary method for the variance of the $\varepsilon$ (upper) and $\boldsymbol{\Gamma}_{1}$ (lower) estimates when the noise power $\xi^{2}$ varies and $N_{1}=N_{2}=$ 20 85

4.19 Results of an occlusive eye image: the output of Canny edge detection (upper left); the extracted iris boundaries (upper right); the estimated ellipses from the proposed estimator (lower left); the estimated ellipses by fitting inner and outer boundaries

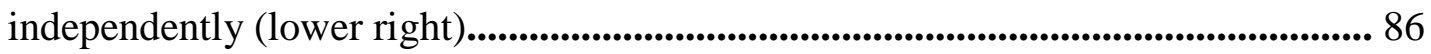

4.20 Results of an eye image with relatively clean detected edges: the output of Canny edge detection (upper left); the extracted iris boundaries (upper right); the estimated ellipses from the proposed estimator (lower left); the estimated ellipses from the O'Leary method (lower right) 
4.21 Results of an eye image with a little noisy detected edges: the output of Canny edge detection (upper left); the extracted iris boundaries (upper right); the estimated ellipses from the proposed estimator (lower left); the estimated ellipses from the O'Leary method (lower right)................................................................................................. 87

4.22 Results of an eye image with quite noisy detected edges: the output of Canny edge detection (upper left); the extracted iris boundaries (upper right); the estimated ellipses from the proposed estimator (lower left); the estimated ellipses from the O'Leary method (lower right)...................................................................................................... 87

5.1 Localization of the source at $\mathbf{u}^{\mathrm{o}}$ using a number of sensors at $\boldsymbol{s}_{\mathrm{i}}$. Open squares denote the true sensor positions that are not known and closed squares are the available sensor positions that are erroneous........................................................................................... 97

5.2 Comparison of the CRLB of a source location estimate in the presence (solid line) and the absence (dash line) of sensor position errors for the distant source. Only the traces of the CRLBs are shown

5.3 Theoretical MSE of the source location estimate from an algorithm that ignores sensor position errors, cross symbol for MSE of the distant source, circle symbol for MSE of the near source, solid lines for CRLB. Only the traces of $\operatorname{MSE}(\mathbf{u})$ and CRLB(u) are shown............................................................................................................. 98

5.4 Performance of the proposed estimator for the distant (up triangle symbol) and the near (down triangle symbol) source with respect to the CRLB (solid line). Only the traces of the CRLBs are shown.............................................................................. 98 
6.1 Localization of the sources at $\mathbf{u}_{i}{ }^{\mathrm{o}}$ using sensors at $\mathbf{s}_{j}$. Open squares denote the true sensor positions that are not known and closed squares are the available sensor positions that are erroneous.......................................................................................... 109

6.2 The 100 random localization geometries, upper triangles represent the sources and

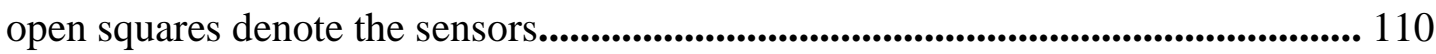

6.3 Comparison of the estimation accuracy when $\sigma_{\mathrm{r}}^{2}$ is fixed to $10^{-3}$ and the sensor position noise power varies. (a) Results on source location estimates. (b) Results on

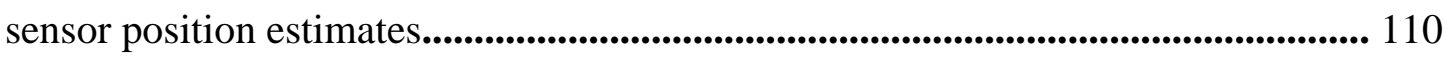

6.4 Estimation results of the new source location when $\sigma_{\mathrm{r}}^{2}$ is fixed to $10^{-3}$ and the sensor position noise power varies........................................................................................................ 111

7.1 Localization scenario. Open circles denote the true sensor positions that are not known and closed circles are the available sensor positions that are erroneous....... 144

7.2 Source location estimate mse results of MLEs considering and ignoring sensor position errors using TOA measurements as $\sigma_{\mathrm{s}}^{2}$ varies

7.3 Source location estimate mse results of MLEs considering and ignoring sensor position errors using TDOA measurements as $\sigma_{\mathrm{s}}{ }^{2}$ varies...................................... 145

7.4 Source location estimate mse results of MLEs considering and ignoring sensor position errors using AOA measurements as $\sigma_{\mathrm{s}}^{2}$ varies............................................... 146

7.5 Source location estimate mse results of MLEs considering and ignoring sensor position errors using TOA measurements as $\sigma_{\mathrm{r}}^{2}$ varies............................................ 146

7.6 Source location estimate mse results of MLEs considering and ignoring sensor position errors using TDOA measurements as ${\sigma_{\mathrm{d}}}^{2}$ varies 
7.7 Source location estimate mse results of MLEs considering and ignoring sensor position errors using AOA measurements as $\sigma_{\mathrm{a}}{ }^{2}$ varies................................................... 147

7.8 Theoretical source location estimate mse for 2-D localization with a calibration source position defined by the axes using TOA measurements, darker (bluer) level represents smaller mse...................................................................................................................... 148

7.9 Source location estimate mse results from the 5 calibration sources using TOA measurements as $\sigma_{\mathrm{s}}^{2}$ varies...................................................................................... 148

7.10 Source location estimate mse results from the 5 calibration sources using TDOA

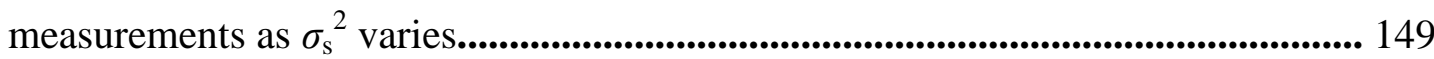

7.11 Source location estimate mse results from the 5 calibration sources using AOA measurements as $\sigma_{\mathrm{s}}^{2}$ varies..................................................................................... 149 


\begin{abstract}
Solving the nonlinear estimation problem is known to be a challenging task because of the implicit relationship between the measurement data and the unknown parameters to be estimated. Iterative methods such as the Taylor-series expansion based ML estimator are presented in this thesis to solve the nonlinear estimation problem. However, they might suffer from the initialization and convergence problems. Other than the iterative methods, this thesis aims to provide a computational effective, asymptotically efficient and closed-form solution to the nonlinear estimation problem.
\end{abstract}

Two kinds of classic nonlinear estimation problems are considered: the geometric shape fitting problem and the source localization problem. For the geometric shape fitting, the research in this thesis focuses on the circle and the ellipse fittings. Three iterative methods for the fitting of a single circle: the ML method, the FLS method and the SDP method, are provided and their performances are analyzed. To overcome the limitations of the iterative methods, asymptotically efficient and closed-form solutions for both the circle and ellipse fittings are derived. The good performances of the proposed solutions are supported by simulations using synthetic data as well as experiments on real images.

The localization of a source via a group of sensors is another important nonlinear estimation problem studied in this thesis. Based on the TOA measurements, the CRLB and MSE results of a source location when sensor position errors are present are derived and compared to show the estimation performance loss due to the sensor position errors. A closed-formed estimator that takes into account the sensor position errors is then proposed. To further improve the sensor position and the source location estimates, an 
algebraic solution that jointly estimates the source and sensor positions is provided, which provides better performance in sensor position estimates at higher noise level comparing to the sequential estimation-refinement technique. The TOA based CRLB and MSE studies are further extended to the TDOA and AOA cases. Through the analysis one interesting result has been found: there are situations exist where taking into account the sensor position errors when estimating the source location will not improve the estimation accuracy. In such cases a calibration emitter with known position is needed to limit the estimation damage caused by the sensor position uncertainties. Investigation has been implemented to find out where would be the optimum position to place the calibration emitter. When the optimum calibration source position may be of theoretical interest only, a practical suboptimum criterion is developed which yields a better calibration emitter position than the closest to the unknown source criterion. 


\section{Chapter 1}

\section{Introduction}

\subsection{Research Areas and Motivations}

The primary object of my research is to provide solutions to the important and challenging nonlinear estimation problem. The nonlinear estimation problem is not trivial to solve because there is only an implicit rather than explicit relationship between the observed data measurements and the unknown parameters to be estimated.

Based on the probability density function (pdf) of the measurement noise, the Maximum Likelihood (ML) estimator can be derived, which is an asymptotically efficient estimator when the signal-to-noise ratio (SNR) is high. However, it is not straightforward to obtain the ML estimator for the nonlinear estimation problem. The Taylor-series expansion can be used to obtain the ML solution for the nonlinear estimation problem via iterations, but its estimation accuracy could be heavily relied on the initial solution guess. Some techniques, such as the semi-definite relaxation (SDR) and the semi definite programming (SDP), can be implemented to the ML cost function and its constraints in order to guarantee an optimum global convergence 
solution, but the trade-off is the dramatic increase in computation.

Having the disadvantages of the ML solution in mind, we intend to apply digital signal processing techniques to the nonlinear estimation problem in order to provide a computational effective, efficient and most importantly, closed-form solution. The solution should not require iteration so that it avoids the initialization problem as well as the convergence issue, and it should not be computationally intensive.

In this thesis we aim to provide new solutions to two important nonlinear estimation problems: the circle and ellipse fittings, and the source localization via a collection of spatially distributed sensors. They are similar to each other in a way that their measurements only have nonlinear relationships with respect to the unknown parameters we are interested in. More details of the nonlinear relationships will be provided in the following chapters.

\subsubsection{Circle and Ellipse Fittings}

The fitting of a geometric shape to a number of noisy data points has been a fundamental and important problem for pattern recognition [1] and object classification [2]. Among different geometric objects, circle and ellipse are the most popular shapes for data fitting because they encompass the shapes of many different objects encountered in practice. For example, the boundaries of a human iris and a mechanical pipe. This classic nonlinear estimation problem continues to attract a broad range of research interests over the years in applications such as biometric matching [3], robotic applications [4] and image processing [5].

We begin the research work in this thesis by examining classic two methods designed to estimate parameters of a circle: the ML method and the Full-Least-Squares 
(FLS) method. The new implementation of the ML estimator in this thesis is based on the noisy model from the data while the FLS method minimizes the geometric distance square. Both methods provide iterative solutions through the Taylor-series linearization technique. The ML estimator has been shown to attain the CRLB accuracy asymptotically [6] and the study in [7] shows that the FLS method gives the ML solution. However, through the comparison of their cost functions, we show analytically that the FLS method approximates the ML estimator only if the noise power is much less than the circle radius square.

Though the Taylor-series linearization approach can be used to obtain the ML solution for the circle fitting problem through iterations, its estimation accuracy could be highly dependent on how it is initialized. Poor initialization may lead to a solution that converges to a local minimum instead of a global minimum. To handle the initialization issue, a SDP solution for the circle fitting problem based on the SDR [8] technique is proposed. In this approach the ML cost function is reformulated and relaxed in order to convert a nonconvex problem to an approximate but convex one. As a result, the SDP method, which guarantees an optimum global convergence, can be implemented to obtain the circle parameter estimates.

Other than the fitting of a single circle or ellipse, the fitting of coupled geometric objects, such as a pair of concentric circles or concentric ellipses, is also an important and challenging problem in the field of reverse engineering [9] and metric vision [10]. Many objects encountered in practice are circularly concentric. A simple example is the inner and outer boundaries of a pipe. Another example is the inner and outer edges of an iris image. When viewing from different angles, concentric circles would become concentric ellipses. The concentric circles and concentric ellipses fitting meth- 
ods proposed in this thesis have potential usages in automatic inspection for pipes, iris recognition for biometric applications, calibration for cameras and ellipticity estimation of steel coils. To solve this nonlinear estimation problem, asymptotically efficient estimators for concentric circle and concentric ellipse fittings based on the weighted equation error formulation and nonlinear parameter transformation are derived. The proposed estimators have explicit solutions and do not require iterations and initial solution guesses. The Cramér-Rao Lower Bound (CRLB) for the parameters of concentric circles and concentric ellipses under Gaussian additive noise are also developed to serve as the performance benchmark. Small-noise analysis is conducted to show that the estimators reach the CRLB accuracy asymptotically.

\subsubsection{Source Localization}

The second nonlinear estimation problem considered in this thesis is the source localization based on measurements from a collection of sensors. This important problem has wild applications in cellular networks, wireless local area networks (WLAN) and sensor networks [11-13]. There are many types of measurements that are commonly used to estimate the source location: the received signal strength (RSS), time-of-arrival (TOA), time-difference-of-arrival (TDOA), angle-of-arrival (AOA) and frequency-difference-of-arrival (FDOA) of a source signal in reaching the sensors [14]. The source localization algorithms proposed in this thesis focus on the TOA measurement, while the CRLB and mean-square error (MSE) studies of the source localization problem in the later chapter cover TOA, TDOA and AOA measurements.

Most existing TOA positioning algorithms do not take into account the uncertainties in sensor positions and assumed they are perfectly known. This assumption is 
very unlikely to be satisfied in practice due to the possible variation or the inaccurate knowledge of sensor positions. Studies [15-17] have been conducted to minimize the decrease in localization performance caused by sensor position uncertainties. However, they neither theoretically analyze the degradation in accuracy of the source location estimate nor provide solutions in closed-form when sensor position errors are present.

In this thesis, based on the TOA measurements, the CRLB of the source location when sensor position errors are present is derived in order to examine the performance decrease. The theoretical increase in the MSE of a source location estimate when the sensor position errors are ignored is also developed. Most importantly, a closed-form solution of the source location that accounts for the sensor position uncertainties is provided. The proposed solution is not iterative and is able to reach the CRLB accuracy.

As shown in the study, the sensor position uncertainty could contribute to considerable amount of degradation in localization accuracy. One can improve the sensor positions by using one or multiple sources, often called calibration sources or anchors, that are at known locations. However, deploying a calibration source could be costly. A more practical approach is to refine the sensor positions upon the localization of an unknown emitting source $[18,19]$. Based on the available, although inexact, sensor positions one can identify the location of the unknown source. The estimated unknown source location can be exploited to refine the inaccurate sensor positions. This is the technique taken in [20] which has shown that such a refinement scheme can achieve the CRLB accuracy for the sensor positions.

The approach in this thesis is different than the previous stated estimation- 
refinement scheme and performs joint estimation of the unknown source locations and the inaccurate sensor positions together. Joint estimation of the positions of the source and sensors for the TDOA measurement has been examined in [21]. The proposed solution extends the method for the TOA case in sensor networks and compares the performance with the sequential estimation-refinement approach. Rather than resorting to the traditional Maximum Likelihood Estimator (MLE) which requires good initialization and high complexity [18] or suboptimum estimator [19], here a computationally efficient algebraic solution is developed. The joint estimation is expected to tolerate higher noise level before the thresholding effect caused by the nonlinear estimation starts to occur.

Besides the TOA based source localization techniques, studies in [13], [18] and [22] also have taken into account the sensor position errors in their source localization methods based on other measurements, such as direction-of-arrival (DOA) and TDOA, in order to improve the localization performance. These investigations on the relation between the sensor position uncertainties and the estimation performance motivate us to extend the CRLB and MSE studies implemented in the TOA source localization solution further to include the TDOA and AOA measurements. After generalizing the CRLB derivation for different types of measurements in the presence of the sensor position errors, we evaluate the MSE of a ML estimator for an arbitrary measurement, pretending the sensor position uncertainties are absent. The MSE evaluation is based on the Taylor-series expansion under small error analysis, which means only linear noise terms are kept in the expansion. This study shows that when the sensor position and measurement noise covariance matrices satisfy certain relationships, one can simply assume the sensor position noise is absent and yet 
achieving the optimum Cramer-Rao Lower Bound (CRLB) [6] performance. This is a rather interesting result and can simplify the localization task when such kind of sensor position and measurement noise occur. Such relationships for TOA, TDOA and AOA positionings are developed. The conditions are derived by obtaining the localization mean-square error matrix of the Maximum Likelihood Estimator (MLE) designed for accurate sensor positions, evaluating the CRLB for joint estimation of source and sensor positions and equating the two.

In the view of performance instead of computation, such kinds of sensor position noise is not preferred since there is no accuracy gained by taking the sensor position statistics into account. Advancing the performance would require the deployment of a calibration emitter whose position is exactly known [22]. This thesis also investigates the optimum placement problem of calibration source. This is accomplished by maximizing the Fisher Information Matrix (FIM), which is the inverse of the CRLB, of the source location estimate with respect to the calibration source position. This is a rather challenging problem and there is no analytical solution in general. Under certain situations such as independent, identically distributed (IID) or very significant sensor position errors compared to measurement noise, the optimum calibration position is shown to be the unknown source position. One would expect, even not necessarily practical, placing the calibration emitter as close to the unknown source as possible would give better performance. This is not necessarily the case from our analysis. We therefore propose a calibration emitter positioning criterion that indeed outperforms the closest to unknown source placement strategy. 


\subsection{Circle and Ellipse Fittings Basics}

In general, the nonlinear estimation problem of circle or ellipse fitting can be stated as follows. Given a collection of $N$ data points in a 2-D plane, we intend to estimate the corresponding center and radius of a circle, or the center, semi-major axis, semi-minor axis and rotation angle of an ellipse. Unlike line fitting, circle or ellipse fitting is a much more challenging task because the nonlinear relationship between the measurements and the unknowns. In this section, we first review the available algorithms and methods for circle and ellipse fittings in literatures. Then, some basic knowledge regarding to the SDR and SDP techniques are introduced.

\subsubsection{Literature Survey}

Many methods for circle fitting have been proposed over the years [7], [23] - [30]. The full-least squares (FLS) method is able to achieve the CRLB performance. It is, however, iterative and numerical solution is needed. The average of intersections (AI) method, the reduced least-squares (RLS) method and the modified least-squares (MLS) method yield closed-form solutions. Their performance, on the other hand, is not able to reach the CRLB accuracy. The Kåsa method [23,24], which has a closedform solution and is simple to apply, appears to be most widely used in practice. It is able to reach the CRLB accuracy when the noise components in $\mathrm{x}$ and $\mathrm{y}$ coordinates are independent and have equal variances (isotropic observation noise) [27,28]. Branch and bound method [29] is proposed for the purpose to obtain maximum likelihood (ML) estimator and its performance is close to the Kåsa's method.

The ellipse fitting problem is more difficult than the circle case and conic formu- 
lation is often used. A few closed-form solutions are presented in [31-33]. Iterative solutions include the Fundamental Numerical Scheme (FNS) [34], the Heteroscedastic Errors-In-Variables (HEIV) method [35,36] and the renormalization method [37]. These iterative methods have better performance than the closed-form solutions and they have been proven analytically to reach the Kanatani-Cramér-Rao (KCR) lower bound [28] accuracy.

All of the above circle and ellipse fitting solutions as well as the KCR lower bound are designed for a single circle or ellipse. Not many literatures are available for the fitting of coupled geometric objects, such as concentric circles and concentric ellipses. Many objects encountered in practice are concentric, a simple example is the inner and outer circles of a DVD. Another example is the inner and outer boundaries of an iris. Kim et al. [38] solved the camera calibration problem by determining the position of a projected circle center through concentric circle formulation. Motivated by determining the original size of the ruined Abhayagiriya stupa, Dampegama [39] provided a specific solution to his problem using concentric circle estimation. [9]- [10] presented more general solutions to the concentric circle/ellipse estimation problems. Benko et al. [9] considered simultaneous fitting of multiple curves and surfaces for reverse engineering. The fitting method they proposed is iterative and requires good initial guesses. Even though it can be extended to the fitting of concentric circles, the work by Marot and Bourennane [40] focused on the radius estimation and the distortion characterization between a circular contour and a circle. O'Leary et al. [10] proposed a quadratically constrained total least squares method for the fitting of coupled geometric objects in metric vision. This method yields closed-form solution when the noise is isotropic but no KCR lower bound performance evaluation was 
given.

\subsubsection{Semi-definite Programming and Relaxation for Circle Fitting}

The concept of SDR was first introduced by Lovász [41] in 1979. In the late 1990s, SDR and SDP are started to be known as powerful tools in solving optimization problem and they have been shown to be computationally efficient by Goemans and Williamson [42]. From the early 2000s, SDR and SDP have been at the center of many research topics in the field of signal processing and communications, such as MIMO detection [43], mobile location [44] and sensor network node localization [15].

A $n \times n$ matrix $\mathbf{X}$ is called positive semi-definite if

$$
\mathbf{v}^{T} \mathbf{X} \mathbf{v} \geq 0 \text { for any } \mathbf{v} \in \mathbf{R}^{n}
$$

In this thesis we use $\mathbf{X} \succeq \mathbf{0}$ to denote that $\mathbf{X}$ is symmetric and positive semi-definite. Let $\mathbf{C}$ also be a symmetric matrix, then a semi-definite program is an optimization problem of the form [45]

$$
\begin{aligned}
\operatorname{minimize} & \mathbf{C} \bullet \mathbf{X} \\
\text { s.t. } & \mathbf{A}_{i} \bullet \mathbf{X}=b_{i}, \quad i=1,2, \cdots, m, \\
& \mathbf{X} \succeq \mathbf{0},
\end{aligned}
$$

where $\mathbf{C} \bullet \mathbf{X}=\sum_{i=1}^{n} \sum_{j=1}^{n} \mathbf{C}_{i j} \mathbf{X}_{i j}$ is a linear function of $\mathbf{X}$.

The circle fitting problem can be considered as nonconvex quadratically constrained quadratic programs (QCQPs) (for details please refer to Chapter 3), which 
can be expressed as [43]

$$
\begin{array}{ll}
\min _{\mathbf{x} \in \mathbf{R}^{n}} & \mathbf{x}^{T} \mathbf{C x} \\
\text { s.t. } & \mathbf{x}^{T} \mathbf{A}_{i} \mathbf{x} \unrhd_{i} b_{i}, \quad i=1,2, \cdots, m,
\end{array}
$$

where $\unrhd_{i}$ denotes $\geq$, =, or $\leq$ for different $i$. Let $\operatorname{Tr}(*)$ be the trace operation, using the matrix property about the trace operation

$$
\begin{aligned}
& \mathbf{x}^{T} \mathbf{C} \mathbf{x}=\operatorname{Tr}\left(\mathbf{x}^{T} \mathbf{C} \mathbf{x}\right)=\operatorname{Tr}\left(\mathbf{C} \mathbf{x} \mathbf{x}^{T}\right) \\
& \mathbf{x}^{T} \mathbf{A}_{i} \mathbf{x}=\operatorname{Tr}\left(\mathbf{x}^{T} \mathbf{A}_{i} \mathbf{x}\right)=\operatorname{Tr}\left(\mathbf{A}_{i} \mathbf{x} \mathbf{x}^{T}\right)
\end{aligned}
$$

and replacing $\mathbf{x x}^{T}$ by $\mathbf{X},(1.3)$ becomes

$$
\begin{array}{ll}
\min & \operatorname{Tr}(\mathbf{C X}) \\
\text { s.t. } & \operatorname{Tr}\left(\mathbf{A}_{i} \mathbf{X}\right) \unrhd_{i} b_{i}, \quad i=1,2, \cdots, m, \\
& \mathbf{X} \succeq \mathbf{0}, \quad \operatorname{rank}(\mathbf{X})=1 .
\end{array}
$$

Now, except the nonconvex rank one constraint, The rest of the constraints and the cost function in (1.5) are all convex in $\mathbf{X}$. If we further relax the rank one constraint on $\mathbf{X}$, we arrive at

$$
\begin{array}{ll}
\min & \operatorname{Tr}(\mathbf{C X}) \\
\text { s.t. } & \operatorname{Tr}\left(\mathbf{A}_{i} \mathbf{X}\right) \unrhd_{i} b_{i}, \quad i=1,2, \cdots, m, \\
& \mathbf{X} \succeq \mathbf{0} .
\end{array}
$$

(1.6) is called the semi definite relaxation of (1.3). In the proposed circle fitting method we shall present in Chapter 3, the ML cost function is re-formulated and 
relaxed using the SDR technique in order to be solved using the SDP method.

\subsection{Source Localization Basics}

Source localization is another classic nonlinear estimation problem considered in this thesis. In this section, we first provide basic knowledge about different types of measurements that are commonly used for source localization. Secondly, a literature survey on the available source localization algorithms and methods that taking into account the sensor position errors is presented. Last but not least, an overview of some of the TOA based localization algorithms is given.

\subsubsection{Source Localization Measurements}

In this subsection, we describe the concepts and applications of three commonly used measurements in source localization: the TOA, AOA and TDOA measurements.

\section{Time of Arrival (TOA)}

The time when the signal from the source arrives at a sensor is the basic element of the TOA measurement. Here we denote $\mathbf{u}^{o}=\left[x^{o}, y^{o}\right]^{T}$ as the Cartesian coordinate vector in column format of the source, and let $\mathbf{s}_{i}^{o}=\left[x_{i}^{o}, y_{i}^{o}\right]^{T}$ be the Cartesian coordinate vector in column format of the sensor $i, i=1,2, \cdots, M$, where $M$ is the total number of sensors. Note that we can convert the time measurements to range measurements by multiplying with the known signal propagation speed $c$. Let $r_{i}^{o}=\left\|\mathbf{u}^{o}-\mathbf{s}_{i}^{o}\right\|$ be the true range between the source $\mathbf{u}^{o}$ and sensor $\mathbf{s}_{i}^{o}$, where $\|\cdot\|$ denotes the Euclidean 
norm. The TOA measurement between the source and sensor $i$ is

$$
r_{i}=r_{i}^{o}+n_{r i}, i=1,2, \cdots, M,
$$

where $n_{r i}$ is the TOA measurement noise. From the definition of $r_{i}^{o}$, we know that each TOA measurement gives a locus of a circle centered at sensor $\mathbf{s}_{i}^{o}$, and the location of the source will be on the circle. As a result, we are able to use the intersection point from three circles that defined by three TOA measurements as the source location in the two dimensional (2D) case. This localization process is shown in Fig. 1.1.

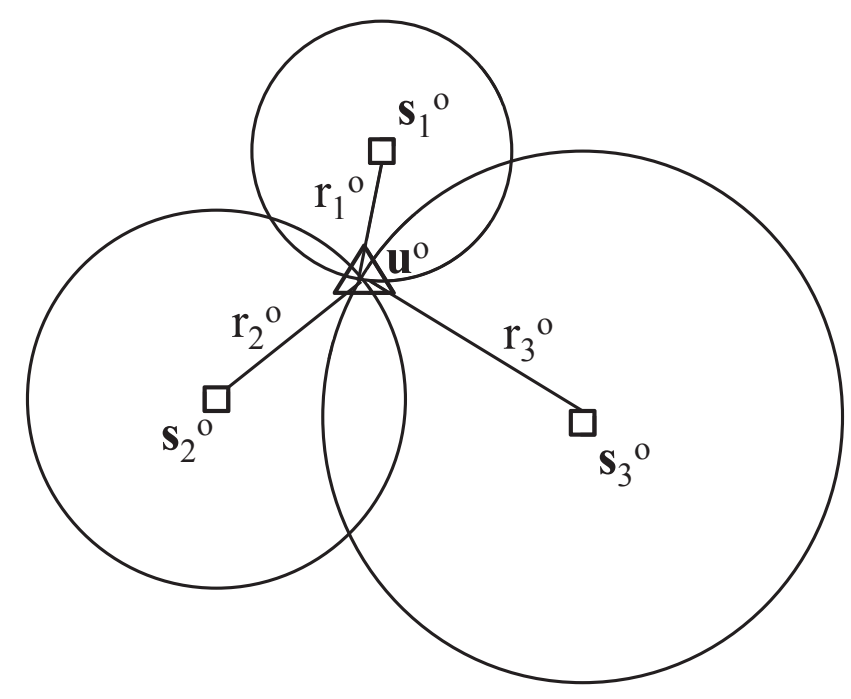

Figure 1.1: Source localization using TOA measurement. The triangle is the source $\mathbf{u}^{o}$. The sensors are denoted as the squares.

\section{Angel of Arrival (AOA)}

The AOA measurement utilizes the bearing between the received source signal and sensors. Let $b_{i}^{o}$ be the true bearing of the source with respect to sensor $i$, the AOA 
measurement is then

$$
b_{i}=b_{i}^{o}+n_{b i}, \quad b_{i}^{o}=\tan ^{-1} \frac{y^{o}-y_{i}^{o}}{x^{o}-x_{i}^{o}}, i=1,2, \cdots, M
$$

where $n_{b i}$ is the bearing noise, $x^{o}$ and $y^{o}$ are the source location, $x_{i}^{o}$ and $y_{i}^{o}$ are the positions of sensor $i$. Each AOA measurement forms a line between the source and sensor $i$. Therefore, by intersecting two lines from two AOA measurements can determine the location of the source, which is shown in Fig. 1.2.

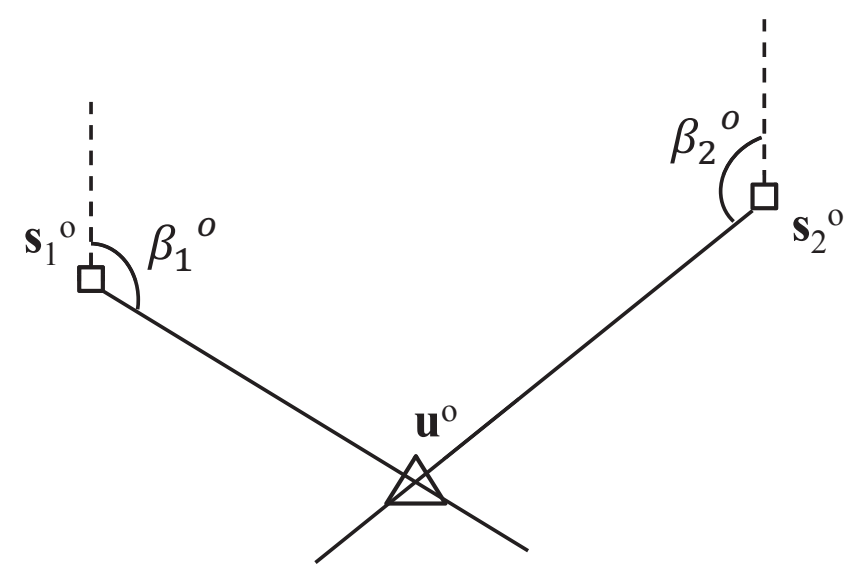

Figure 1.2: Source localization using AOA measurement. The triangle is the source $\mathbf{u}^{o}$. The sensors are denoted as the squares.

\section{Time Difference of Arrival (TDOA)}

As the name implies, the TDOA measurement provides the difference of the arrival times of the source signal when it reaches a pair of sensors. As in the TOA case, the time difference can be converted to range difference. By using $\mathbf{s}_{1}^{o}$ as the reference sensor, the TDOA measurement from the source $\mathbf{u}^{o}$ to the sensor pair $i$ and 1 , after 
multiplying with the signal propagation speed, is

$$
r_{i 1}=r_{i 1}^{o}+n_{i 1}, i=2,3, \cdots, M
$$

where $r_{i 1}^{o}=r_{i}^{o}-r_{1}^{o}$ and $n_{i 1}$ is the TDOA measurement noise. For each TDOA measurement and the corresponding sensor pair, the source will be located at one half of a hyperbola whose foci are the two sensors. In Fig. 1.3, we shown that in the $2 \mathrm{D}$ case, the intersection point of the two hyperbolas from two TDOA measurements is the location of the source.

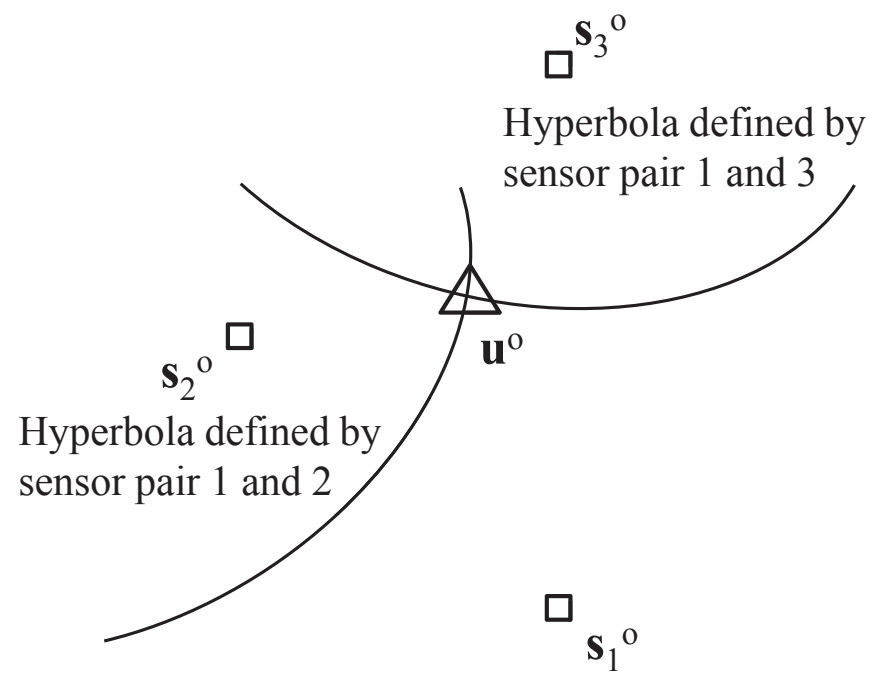

Figure 1.3: Source localization using TDOA measurement. The triangle is the source $\mathbf{u}^{o}$. The sensors are denoted as the squares. 


\subsubsection{Literature Survey of Source Localization Methods}

There are many factors that affect the localization accuracy. Some of them can be taken into account during the system design, such as the number and the arrangement of sensors. Others are often not possible to control, such as the received signal-to-noise ratios (SNRs), the multipath propagations and the sensor position uncertainties. Traditional localization system, such as in radar, has fixed receivers at designated locations with direct line of sight reception and the performance is pretty much determined by the SNRs. In wireless communications and indoor localization, multipath reflections occur very common and they introduce bias to the signal arrival times at the sensors [46]. The TOA and TDOA containing multipath propagation could degrade the localization performance significantly and they need to be filtered out [47]. Modern localization uses mobile or randomly deployed sensors and their precise positions are not known exactly, which causes reduction in estimation accuracy $[13]$.

Several studies have been conducted to minimize the decrease in localization performance caused by sensor position errors. In the direction-of-arrival (DOA) estimation, Rockah and Schultheiss [18] showed that for the far-field scenario when the sensor positions are erroneous, major performance gain can be achieved through array shape calibration. Chen et al. [48] estimates the AOA using Toeplitz approximation in the presence of sensor position errors. However, these methods focus on the estimation of DOA measurements, not the estimate of source location. Ho et al. [13] analyzed the performance degradation due to sensor position errors for TDOA positioning and developed a closed-form solution to improve the source location estimate. Recently, Ho and Yang [22] used a calibration source at exactly known position to 
improve the TDOA localization performance further in the presence of sensor position uncertainties.

In the case of TOA localization, Srirangarajan et al. [16] presented a second order cone programming (SOCP) approach to solve the problem with noisy distance measurements and inaccurate anchor positions. Lui et al. [15] devised SDP algorithms for node localization in the presence of uncertainties in anchor positions and/or propagation speed. Zheng and $\mathrm{Wu}[17]$ also proposed a method that jointly solves the source location and the time synchronization with bounded anchor uncertainties. However, these methods didn't theoretically analyze the degradation in accuracy of the source location estimate when sensor position errors are present. Also, none of them provide closed-form solutions.

\subsubsection{Overview of TOA Source Localization Methods}

Because of the nonlinear relation between the source location and the TOA measurement, it is non trivial to solve the TOA source localization problem. By assuming the distribution of the TOA measurement noise is available, the source localization problem can be solved using the ML algorithm such as the one in [11]. However, even though the ML algorithms could reach the CRLB accuracy asymptotically, they are normally iterative and require an initial guess of the source location. As a result, local minima instead of global minima are very likely to occur if the initial solution guess is not chosen carefully. To handle this issue, some algorithms $[15,16]$ based on SOCP and SDP are proposed. With increases in computation, these methods can provide optimum global convergence solutions.

The CRLB establishes a lower bound on the error covariance matrix for any deter- 
ministic unbiased estimator [6]. Empirically, it is also an accurate performance limit for a nonlinear estimation problem over the small noise region in which the bias is insignificant. The CRLB is usually served as a benchmark to evaluate the performance of the TOA source localization methods. When a source localization estimator gives an unbiased source location estimate with a covariance matrix that is equal to the CRLB, we consider this estimator as an efficient estimator. Or when the estimate covariance matrix is approximately equal to the CRLB, we called it approximately efficient. With accurate sensor position measurements, most existing TOA algorithms are (approximately) efficient and are able to reach the CRLB accuracy within certain noise range.

The proposed TOA source localization algorithms in this thesis taking into account the sensor position errors when estimating the source location and provide closed form solutions. They does not suffer from the initialization problem as in the ML estimator. They also has been shown analytically to reach the CRLB accuracy in the presence of sensor position errors.

\subsection{Organization of the Thesis}

In this section we provide a brief description of the research works included in this thesis. The two research topics considered here are the circle and ellipse fittings problem (Chapter 2 to Chapter 4), and the source localization problem (Chapter 5 to Chapter 7).

Chapter 2 focuses on a new implementation of the ML estimator based on the noisy model from the data through the Taylor-series linearization technique, as well as the 
comparison of the ML estimator and the FLS method. The theoretical investigation indicates that the FLS method is not the ML estimator. This is in contrast to the previous study [7] stating that the FLS method gives the ML solution. We have shown that the FLS method approximates the ML estimator only when the noise power of the measurements is much smaller than the radius square of the circle. Hence, we arrive at the conclusion that the ML solution outperforms the FLS method when the noise power is large or when the circle radius is small. The theoretical study is supported by simulations at the end of the chapter.

The ML solution presented in Chapter 2 is based on the Taylor-series linearization and its solution needs to be obtained through iterations. As a result, its estimation performance is highly dependent on the initial solution guess. In order to handle this problem, in Chapter 3 we propose another implementation of the ML estimator using the SDR and SDP techniques. We re-formulate the ML cost function and the constraints in order to implement the semi-definite relaxation. After the relaxation, the nonconvex estimation problem is converted to an approximate but convex one, and can be solved using the SDP method, which provides an optimum global convergence solution. At the end of the chapter, the performance of the proposed estimator is shown to have the CRLB accuracy and is compared with the well-known Käsa method.

To the best of the author's knowledge, not many studies in literature are available for the fittings of concentric circles and concentric ellipses. In Chapter 4 we develop new solutions for the fittings of concentric circles and concentric ellipses without requiring uncorrelated behavior of the noise. The proposed estimator is based on nonlinear transformation of the unknown parameters to be estimated through the 
formulation of weighted equation error minimization. The proposed solutions have explicit forms. It can also produce a non-iterative solution but the performance is suboptimal. Self-initialized iterative refinement can improve the estimation accuracy to optimum. To validate the efficient performance of the proposed solutions, we also provide the KCR lower bounds for the concentric circle and concentric ellipse fitting problems. The proposed concentric circle and concentric ellipse estimators are different from the previous solutions in [38]- [39] which are specific to their particular problems. More importantly, comparing to [10] the proposed estimator is shown analytically and validated by simulations to achieve the optimum KCR lower bound performance asymptotically (when the measurement noise is small). When contrasting to FNS and HEIV methods extended to coupled object fittings, the proposed estimators have higher noise tolerance levels before the thresholding effects take place. The proposed methods can be reduced back to the fittings of a single circle and a single ellipse. In such cases, they maintain the KCR lower bound performance for both isotropic and anisotropic noise. Anisotropic noise, meaning that the noise components in the $\mathrm{x}$ and y coordinate points are correlated, occurs quite often in practice such as in robotics and archaeology $[4,30]$. In the end of the chapter, we implement the proposed fitting method on the iris recognition application using real images.

For the source localization problem, based on the TOA measurement, in Chapter 5 we examine the decrease in the source localization accuracy due to the sensor position errors. The examination is done by the comparing the CRLB of the source location when sensor position errors are present with the MSE of a source location estimate when the sensor position errors are ignored but in fact exist. The contrast of the CRLB and the MSE indicates how much the performance degradation is due 
to the sensor position errors. In order to improve the source location accuracy in the presence of sensor position errors, a closed-form solution that can achieve the CRLB performance is developed. Simulations are included to support the theoretical development.

In modern localization systems/platforms such as sensor networks, improving the sensor positions is necessary in order to achieve better localization performance. In Chapter 6 a joint estimator for locating multiple unknown sources and refining the sensor positions using TOA measurements is proposed. Rather than resorting to the traditional iterative nonlinear least-squares approach that requires careful initializations, the proposed estimator is algebraic and computationally attractive. The small noise analysis shows that the proposed estimator is able to attain the CRLB performance for both the unknown sources and the sensor positions. Simulations support the efficiency of the proposed estimator.

The studies in Chapters 5 and 6 have shown that an estimator would require the use of the statistical knowledge of the sensor positions errors in order to reach the optimum localization performance. Chapter 7 shows that when the covariance matrices of the sensor position and the measurement noise satisfy certain relationships, taking the sensor position error into account is not necessary and a simpler estimator that pretends the sensor position uncertainties are absent is sufficient to reach the optimum performance. Further accuracy improvement necessitates a calibration emitter whose position is known exactly to correct the sensor positions. It is known that the performance gain from a calibration emitter depends on where it is placed. We derive the optimum calibration position by maximizing the Fisher Information of the source location estimate. The optimum position is of theoretical interest and may not be 
practical. A suboptimum criterion for realistic calibration emitter placement is then proposed. We shall use TOA, TDOA and AOA localizations to illustrate the derived results. Simulations support the theoretical developments and the performance analysis.

Chapter 8 summaries the report and discusses the ideas of future research works.

\subsection{Major Contributions}

In this section, the major contributions of this thesis is listed as follow:

a) A new Maximum likelihood (ML) estimator based on Taylor-series linearization under Gaussian white noise is developed and compared with the Full least-square (FLS) method. Unlike the result from a previous work [7], it has be shown here that the FLS method only approximates the ML estimator if the ratio between noise power and circle radius square is much less than unity.

b) The semi-definite programming (SDP) and the semi-definite relaxation (SDR) techniques are applied to the circle fitting problem. The SDP and SDR are two powerful tools in solving optimization problem and they have been shown to be computationally efficient. In this thesis the ML cost function and constraints are re-formulated and relaxed so that the nonlinear estimation problem can be solved using a new SDP method, which provides the optimum global convergence solution.

c) Computational efficient solutions for concentric circles and concentric ellipses fittings are derived. The new estimators have explicit forms and can produce both non-iterative and iterative solutions. The non-iterative solution has better computational efficiency than the other existing non-iterative methods. The iterative solution 
is self-initialized and provides optimum performance. It also has higher noise tolerance level for the thresholding effect comparing to other existing iterative methods. The new estimators can be reduced back to the fitting of a single circle and a single ellipse.

d) For the research regarding to the source localization in the presence of sensor position noise, based on the TOA measurement the degradation in accuracy of the source location estimate is analyzed theoretically. The analysis indicates how sensitive is the source location estimate with respect to the sensor position errors. A new closed-form solution that accounts the sensor position errors and achieves the CRLB performance is derived.

e) A joint estimator for locating multiple unknown sources and refining the erroneous sensor positions using TOA measurements is proposed. Rather than resorting to the traditional iterative nonlinear ML estimator that requires careful initializations and high complexity, the new estimator is algebraic and computationally attractive. Other than the estimation of the source locations, the proposed method can refine the inaccurate sensor positions which can improve the localization accuracy of newly appeared sources subsequently.

f) It is common believe that ignoring the uncertainties of sensor position when estimating the source location will result in non-optimum estimation performance. However, with further investigation this thesis shows the relations of the sensor position and measurement noise covariance matrices for TOA, TDOA and AOA localizations, under which taking into the sensor position errors into account is not necessary and does not improve the localization accuracy. In such cases, a calibration emitter with known position is needed to limit the damage due to the sensor position errors. 
Its optimum placement position is derived for the TOA, TDOA and AOA measurements with independent, identical distributed (IID) or very significant sensor position noise relative to the measurement errors. When the optimum calibration placement may not be possible in practice, a suboptimal but practical calibration placement criterion is provided. 


\section{Chapter 2}

\section{Maximum Likelihood and Full}

\section{Least-squares Estimators for Circle}

\section{Fitting}

\subsection{The Circle Fitting Problem}

Let $\mathbf{s}_{i}=\left[x_{i}, y_{i}\right]^{T}, i=1,2, \cdots, N$, be a set of $N$ measurement points defined as

$$
\mathbf{s}_{i}=\mathbf{s}_{i}^{o}+\mathbf{n}_{i}
$$

where $\mathbf{s}_{i}^{o}=\left[x_{i}^{o}, y_{i}^{o}\right]^{T}$ is the true data point sampled from a circle of center $\mathbf{c}^{o}=\left[\bar{x}^{o}, \bar{y}^{o}\right]^{T}$ and radius $r^{o}$ such that it satisfies

$$
\left\|\mathbf{s}_{i}^{o}-\mathbf{c}^{o}\right\|=r^{o}
$$


and $\|*\|$ is the Euclidean norm. $\mathbf{n}_{i}$ is the measurement noise and is modeled as zero mean Guassian with diagonal covariance matrix $\sigma^{2} \mathbf{I}_{2 \times 2}$. It is further assumed that $\mathbf{n}_{i}$ is I.I.D. for $i=1,2, \cdots, N$. Given the noisy measurements $\mathbf{s}_{i}$, we are interested to find an estimate of $\boldsymbol{\theta}^{o}=\left[\mathbf{c}^{o T}, r^{o}\right]^{T}$ that best fits the measurements in some optimal sense.

\subsection{The ML Solution}

Since $\mathbf{n}_{i} \sim N\left(0, \sigma^{2} \mathbf{I}_{2 \times 2}\right)$, the ML cost function is simply equal to

$$
J(\boldsymbol{\theta})=\sum_{i=1}^{N}\left\|\mathbf{s}_{i}-\mathbf{s}_{i}^{o}(\boldsymbol{\theta})\right\|^{2}
$$

and the ML solution is the value of $\boldsymbol{\theta}$ that minimizes $J(\boldsymbol{\theta})$. The ML cost function is not quadratic with respect to $\boldsymbol{\theta}$ because $\mathbf{s}_{i}^{o}(\boldsymbol{\theta})$ is related to $\boldsymbol{\theta}$ in a highly nonlinear manner as shown in (2.2). We shall propose the use of Taylor-series linearization approach to minimize $J(\boldsymbol{\theta})$ through iteration.

Let $\boldsymbol{\theta}_{(o)}=\left[\bar{x}_{(o)}, \bar{y}_{(o)}, r_{(o)}\right]^{T}$ be an initial solution guess. Expanding $\mathbf{s}^{o}(\boldsymbol{\theta})$ through Taylor-series up to linear term gives

$$
\mathbf{s}_{i}^{o}(\boldsymbol{\theta})=\mathbf{s}_{i}^{o}\left(\boldsymbol{\theta}_{(o)}\right)+\mathbf{G}_{i}\left(\boldsymbol{\theta}_{(o)}\right)\left(\boldsymbol{\theta}-\boldsymbol{\theta}_{(o)}\right)
$$

where $\mathbf{G}_{i}\left(\boldsymbol{\theta}_{(o)}\right)=\left.\frac{\partial \mathbf{s}_{i}^{o}(\boldsymbol{\theta})}{\partial \boldsymbol{\theta}}\right|_{\boldsymbol{\theta}_{(o)}}$ is the $2 \times 3$ gradient matrix. Putting (2.4) into (2.3) forms

$$
J(\boldsymbol{\theta}) \simeq \sum_{i=1}^{N}\left\|\mathbf{s}_{i}-\mathbf{s}_{i}^{o}\left(\boldsymbol{\theta}_{(o)}\right)-\mathbf{G}_{i}\left(\boldsymbol{\theta}_{(o)}\right)\left(\boldsymbol{\theta}-\boldsymbol{\theta}_{(o)}\right)\right\|^{2}
$$


(2.5) is quadratic with respect to $\boldsymbol{\theta}$, whose minimum is achieved when

$$
\boldsymbol{\theta}=\boldsymbol{\theta}_{(o)}+\left[\sum_{i=1}^{N} \mathbf{G}_{i}\left(\boldsymbol{\theta}_{(o)}\right)^{T} \mathbf{G}_{i}\left(\boldsymbol{\theta}_{(o)}\right)\right]^{-1} \times\left[\sum_{i=1}^{N} \mathbf{G}_{i}\left(\boldsymbol{\theta}_{(o)}\right)^{T}\left(\mathbf{s}_{i}-\mathbf{s}_{i}^{o}\left(\boldsymbol{\theta}_{(o)}\right)\right)\right] .
$$

To improve the solution, we set $\boldsymbol{\theta}_{(o)}$ to the answer from (2.6) and repeat the computation. Indeed, the proposed solution can be easily expressed as

$$
\boldsymbol{\theta}_{(k+1)}=\boldsymbol{\theta}_{(k)}+\left[\sum_{i=1}^{N} \mathbf{G}_{i}\left(\boldsymbol{\theta}_{(k)}\right)^{T} \mathbf{G}_{i}\left(\boldsymbol{\theta}_{(k)}\right)\right]^{-1} \times\left[\sum_{i=1}^{N} \mathbf{G}_{i}\left(\boldsymbol{\theta}_{(k)}\right)^{T}\left(\mathbf{s}_{i}-\mathbf{s}_{i}^{o}\left(\boldsymbol{\theta}_{(k)}\right)\right)\right]
$$

for $k=0,1, \cdots$, where $k$ is the iteration count. The iteration stops when $\| \boldsymbol{\theta}_{(k+1)}$ $\boldsymbol{\theta}_{(k)} \|<\delta$, where $\delta$ is some small number.

We now determine $\mathbf{s}_{i}^{o}\left(\boldsymbol{\theta}_{(k)}\right)$ and $\mathbf{G}_{i}\left(\boldsymbol{\theta}_{(k)}\right)$ to complete the iterative solution. Using Chan \& Thomas [49] parametric form of circle representation, a point $\left(x_{i}^{o}, y_{i}^{o}\right)$ on a circle can be expressed as

$$
x_{i}^{o}=\bar{x}^{o}+r^{o} \cos \left(\phi_{i}^{o}\right), y_{i}^{o}=\bar{y}^{o}+r^{o} \sin \left(\phi_{i}^{o}\right)
$$

where $\phi_{i}^{o}=\tan ^{-1} \frac{y_{i}^{o}-\bar{y}^{o}}{x_{i}^{o}-\bar{x}^{o}}$. Thus, given $\boldsymbol{\theta}_{(k)}=\left[\bar{x}_{(k)}, \bar{y}_{(k)}, r_{(k)}\right]^{T}$,

$$
\begin{aligned}
\mathbf{s}_{i}^{o}\left(\boldsymbol{\theta}_{(k)}\right) & =\left[\begin{array}{c}
\bar{x}_{(k)} \\
\bar{y}_{(k)}
\end{array}\right]+r_{(k)}\left[\begin{array}{c}
\cos \left(\phi_{i}\right) \\
\sin \left(\phi_{i}\right)
\end{array}\right] \\
\phi_{i} & =\tan ^{-1} \frac{y_{i}-\bar{y}_{(k)}}{x_{i}-\bar{x}_{(k)}} .
\end{aligned}
$$

Note that we have replaced $\left(x_{i}^{o}, y_{i}^{o}\right)$ by $\left(x_{i}, y_{i}\right)$ in obtaining $\phi_{i}$ because $\left(x_{i}^{o}, y_{i}^{o}\right)$ is not available. 
The gradient matrix $\mathbf{G}_{i}\left(\boldsymbol{\theta}_{(k)}\right)$ is

$$
\left.\frac{\partial \mathbf{s}_{i}^{o}(\boldsymbol{\theta})}{\partial \boldsymbol{\theta}}\right|_{\boldsymbol{\theta}_{(k)}}=\left.\left[\begin{array}{lll}
\frac{\partial x_{i}^{o}}{\partial \bar{x}} & \frac{\partial x_{i}^{o}}{\partial \bar{y}} & \frac{\partial x_{i}^{o}}{\partial r} \\
\frac{\partial y_{i}^{o}}{\partial \bar{x}} & \frac{\partial y_{i}^{o}}{\partial \bar{y}} & \frac{\partial y_{i}^{o}}{\partial r}
\end{array}\right]\right|_{\boldsymbol{\theta}_{(k)}}
$$

whose elements are [49], after replacing $\left(x_{i}^{o}, y_{i}^{o}\right)$ by $\left(x_{i}, y_{i}\right)$ because $\left(x_{i}^{o}, y_{i}^{o}\right)$ is not known,

$$
\begin{aligned}
& \left.\frac{\partial x_{i}^{o}}{\partial \bar{x}}\right|_{\boldsymbol{\theta}_{(k)}}=\frac{\left(x_{i}-\bar{x}_{(k)}\right)^{2}}{r_{(k)}^{2}},\left.\frac{\partial x_{i}^{o}}{\partial \bar{y}}\right|_{\boldsymbol{\theta}_{(k)}}=\frac{\left(x_{i}-\bar{x}_{(k)}\right)\left(y_{i}-\bar{y}_{(k)}\right)}{r_{(k)}^{2}} \\
& \left.\frac{\partial y_{i}^{o}}{\partial \bar{y}}\right|_{\boldsymbol{\theta}_{(k)}}=\frac{\left(y_{i}-\bar{y}_{(k)}\right)^{2}}{r_{(k)}^{2}},\left.\frac{\partial y_{i}^{o}}{\partial \bar{x}}\right|_{\boldsymbol{\theta}_{(k)}}=\frac{\left(x_{i}-\bar{x}_{(k)}\right)\left(y_{i}-\bar{y}_{(k)}\right)}{r_{(k)}^{2}} \\
& \left.\frac{\partial x_{i}^{o}}{\partial r}\right|_{\boldsymbol{\theta}_{(k)}}=\frac{x_{i}-\bar{x}_{(k)}}{r_{(k)}},\left.\quad \frac{\partial y_{i}^{o}}{\partial r}\right|_{\boldsymbol{\theta}_{(k)}}=\frac{y_{i}-\bar{y}_{(k)}}{r_{(k)}}
\end{aligned}
$$

(2.7) together with (2.9)-(2.11) forms the ML iterative solution.

\subsection{The FLS Solution}

The cost function of FLS is [24]

$$
F=\sum_{i=1}^{N}\left(r-\left\|\mathbf{s}_{i}-\mathbf{c}\right\|\right)^{2} .
$$

The solution $\boldsymbol{\theta}=\left[\mathbf{c}^{T}, r\right]^{T}$ is found by minimizing $F$. It is related to $\boldsymbol{\theta}$ in a complicated manner and iterative minimization is needed. Following the same approach as in the previous section through Taylor-series linearization, the FLS solution through 
iteration is

$$
\boldsymbol{\theta}_{(k+1)}=\boldsymbol{\theta}_{(k)}+\left[\sum_{i=1}^{N} \mathbf{H}_{i}\left(\boldsymbol{\theta}_{(k)}\right)^{T} \mathbf{H}_{i}\left(\boldsymbol{\theta}_{(k)}\right)\right]^{-1} \times\left[\sum_{i=1}^{N} \mathbf{H}_{i}\left(\boldsymbol{\theta}_{(k)}\right)^{T}\left(r_{(k)}-\left\|\mathbf{s}_{i}-\mathbf{c}_{(k)}\right\|\right)\right]
$$

for $k=0,1, \cdots$, where $\boldsymbol{\theta}_{(o)}=\left[\mathbf{c}_{(o)}^{T}, r_{(o)}\right]^{T}$ is some initial guess. The iteration stops when $\left\|\boldsymbol{\theta}_{(k+1)}-\boldsymbol{\theta}_{(k)}\right\|<\delta$, where $\delta$ is a small value. The gradient matrix $\mathbf{H}_{i}\left(\boldsymbol{\theta}_{(k)}\right)$ is

$$
\mathbf{H}_{i}\left(\boldsymbol{\theta}_{(k)}\right)=\left[\left(\frac{\mathbf{s}_{i}-\mathbf{c}_{(k)}}{\left\|\mathbf{s}_{i}-\mathbf{c}_{(k)}\right\|}\right)^{T} 1\right]
$$

\subsection{Comparison}

Let us now compare ML and FLS. We begin with the FLS cost function in (2.12). Expanding the square gives

$$
\left(r-\left\|\mathbf{s}_{i}-\mathbf{c}\right\|\right)^{2}=r^{2}+\left\|\mathbf{s}_{i}-\mathbf{c}\right\|^{2}-2 r\left\|\mathbf{s}_{i}-\mathbf{c}\right\|
$$

Let $\mathbf{s}_{i}^{o}(\boldsymbol{\theta})$ be a point on the circle defined by $\boldsymbol{\theta}$. Then

$$
r^{2}=\left\|\mathbf{s}_{i}^{o}(\boldsymbol{\theta})-\mathbf{c}\right\|^{2}
$$

we can express $\left\|\mathbf{s}_{i}-\mathbf{c}\right\|^{2}$ as

$$
\begin{aligned}
\left\|\mathbf{s}_{i}-\mathbf{c}\right\|^{2} & =\left\|\mathbf{s}_{i}^{o}(\boldsymbol{\theta})-\mathbf{c}+\mathbf{s}_{i}-\mathbf{s}_{i}^{o}(\boldsymbol{\theta})\right\|^{2} \\
& =r^{2}\left[1+\frac{\left\|\mathbf{s}_{i}-\mathbf{s}_{i}^{o}(\boldsymbol{\theta})\right\|^{2}}{r^{2}}+\frac{2}{r^{2}}\left(\mathbf{s}_{i}^{o}(\boldsymbol{\theta})-\mathbf{c}\right)^{T}\left(\mathbf{s}_{i}-\mathbf{s}_{i}^{o}(\boldsymbol{\theta})\right)\right] .
\end{aligned}
$$


If $r$ is large compared to $\left\|\mathbf{s}_{i}-\mathbf{s}_{i}^{o}(\boldsymbol{\theta})\right\|$ so that $\left\|\mathbf{s}_{i}-\mathbf{s}_{i}^{o}(\boldsymbol{\theta})\right\| / r \ll 1$, then from (2.17),

$$
\left\|\mathbf{s}_{i}-\mathbf{c}\right\| \simeq r\left[1+\frac{1}{r^{2}}\left(\mathbf{s}_{i}^{o}(\boldsymbol{\theta})-\mathbf{c}\right)^{T}\left(\mathbf{s}_{i}-\mathbf{s}_{i}^{o}(\boldsymbol{\theta})\right)\right]
$$

Putting (2.17)-(2.18) into (2.15) yields immediately

$$
\left(r-\left\|\mathbf{s}_{i}-\mathbf{c}\right\|\right)^{2} \simeq\left\|\mathbf{s}_{i}-\mathbf{s}_{i}^{o}(\boldsymbol{\theta})\right\|^{2}
$$

so that (2.12) becomes

$$
F \simeq \sum_{i=1}^{N}\left\|\mathbf{s}_{i}-\mathbf{s}_{i}^{o}(\boldsymbol{\theta})\right\|^{2}
$$

which is the ML cost function in (2.3).

We can now conclude that in general FLS is not the same as ML estimator. It approaches the ML estimator if

$$
\varepsilon_{i}=\frac{\left\|\mathbf{s}_{i}-\mathbf{s}_{i}^{o}(\boldsymbol{\theta})\right\|}{r} \ll 1
$$

This condition is satisfied if the noise level in the data measurements is small, or when $r$ is big. Thus, we expect that the ML solution will outperforms the FLS method when the noise level is high or when the radius of the circle is small.

A previous work [7] shows that FLS gives the ML solution. However, it was based on the assumption that $\mathbf{c}$ and $\phi_{i}$ are independent variables. This is not the case as can be inferred from (2.8). 


\subsection{Simulation}

We shall investigate the performance of the proposed ML solution and the FLS method via simulation. The $N$ true data points are sampled from an arc of a circle with radius $r$ and range from 0 to $\beta$. The center of the circle is set to be $(80,60) \mathrm{m}$. The noisy measurements are generated by adding to the true data points zero mean Gaussian white noise with covariance matrix $\sigma^{2} \mathbf{I}_{2 N \times 2 N}$. The estimation accuracy is defined as $\operatorname{MSE}(\boldsymbol{\theta})=\sum_{l=1}^{L}\left\|\boldsymbol{\theta}_{l}-\boldsymbol{\theta}^{\circ}\right\|^{2} / L$. where $\boldsymbol{\theta}_{l}$ is the estimated unknown vector at ensemble $l$ and $L=10000$ is the number of ensemble runs.

Fig. 2.1 shows the results when $N=5, r=10 m$ and $\beta=\pi / 3$. The MSEs of ML and FLS methods, together with the CRLB are shown as function of noise power $\sigma^{2}$. When $\sigma^{2}$ is less than or equal to -25 in log scale, both methods achieve the CRLB accuracy. After $\sigma^{2}$ reaches -20 in log scale, the performance of FLS method suffers from the thresholding effect, while the ML estimator remains to generate seasonable estimates of $\boldsymbol{\theta}^{\circ}$. This observation is consistent with our theoretical analysis in section 5 that for large noise power, the ML solution will outperform the FLS method.

Fig. 2.2 gives the results when $N=5, r=10 \mathrm{~m}$ and $\beta=2 \pi$. The trend observed is similar as in Fig. 2.1. The MSEs of both methods are smaller because the data points are distributed in the whole circle instead of clustering on a small arc.

Fig. 2.3 depicts the averaged fitted circles from five noisy measurement points. Simulation configuration is the same as in Fig. 2.2 except that $\sigma^{2}$ is fixed at -3 in log scale. We can see the circle estimated by ML solution is very close to the true one while the FLS circle significantly deviates from it. This again verifies the theoretical development that FLS method would not behave as an ML estimator when the noise power becomes large. 
Fig. 2.4 illustrated the MSEs of ML and FLS method when $N=20, r=10 \mathrm{~m}$ and $\beta=2 \pi$. The figure indicates that both ML and FLS have lower estimation MSE as

$N$ increases. Both methods deviate gradually from the CRLB when $\sigma^{2}$ is larger than 0 in log scale but no threshold effect occurs.

In generating Fig. 2.5, the parameters are $N=20, r=2 m$ and $\beta=2 \pi$. It is evident that when the radius of the circle decreases, the ML estimator gives much better result than the FLS method after $\sigma^{2}$ reaches 0 in log scale. This observation verifies the result in (2.21) that only when the ratio between noise power and circle radius square is much less than unity, the FLS approximates the ML estimator.

\subsection{Concluding Remarks}

This chapter derives the ML solution for circle fitting using Taylor-series linearization approach, where the noise in the data measurements are Gaussian and white. It then provides a comparison in performance between the ML estimator and the FLS method. Unlike the result from a previous work that illustrates FLS gives the ML solution, we have shown analytically that FLS does not give ML estimation. It approximates the ML estimator if the ratio between noise power and circle radius square is much less than unity. Otherwise the ML estimator gives much better results. Simulations confirm the theoretical findings. 


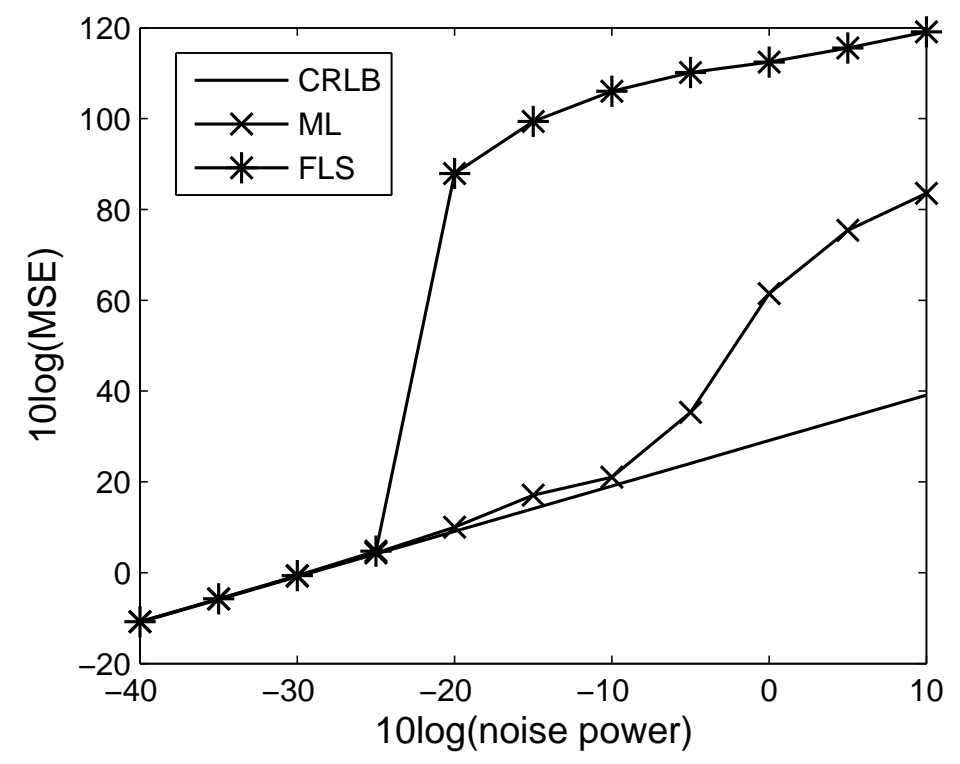

Figure 2.1: Performance comparison of the ML and FLS method when $N=5, r=10$ and $\beta=\pi / 3$.

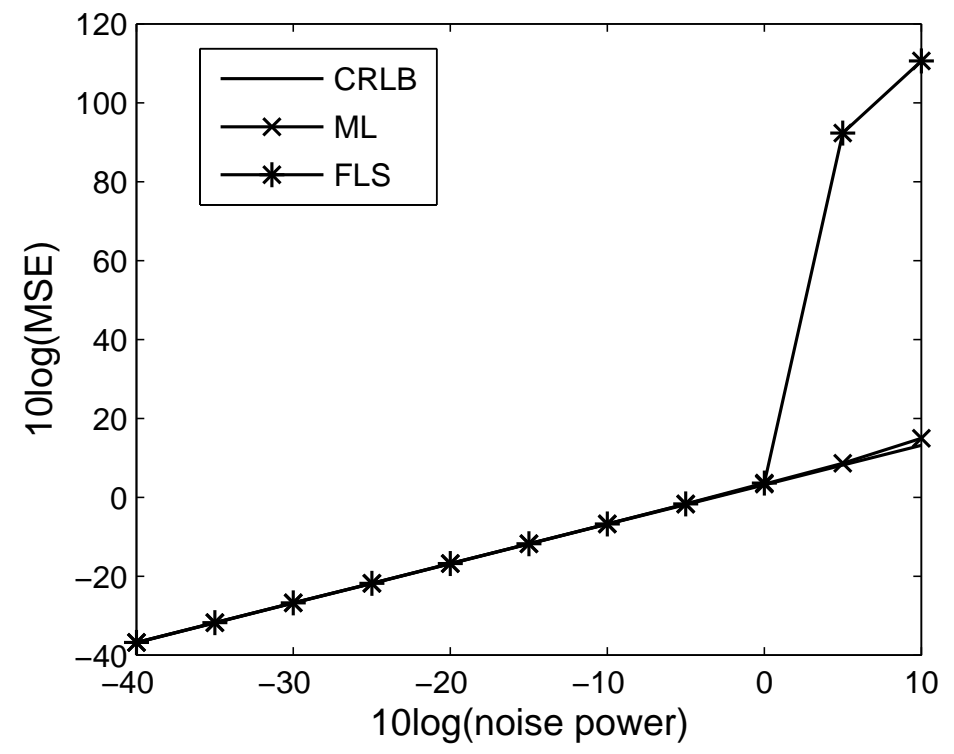

Figure 2.2: Performance comparison of the ML and FLS method when $N=5, r=10$ and $\beta=2 \pi$. 


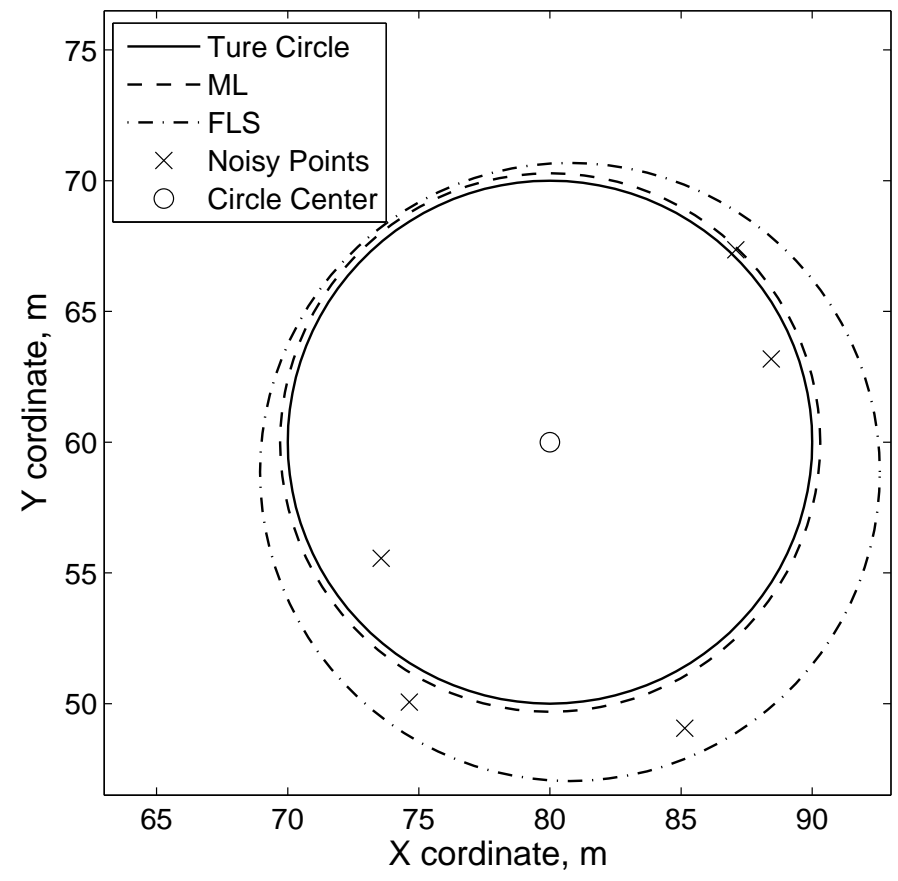

Figure 2.3: Averaged fitted circles of the ML and FLS method when $N=5, r=$ $10, \beta=2 \pi$ and $\sigma^{2}=3 d B$.

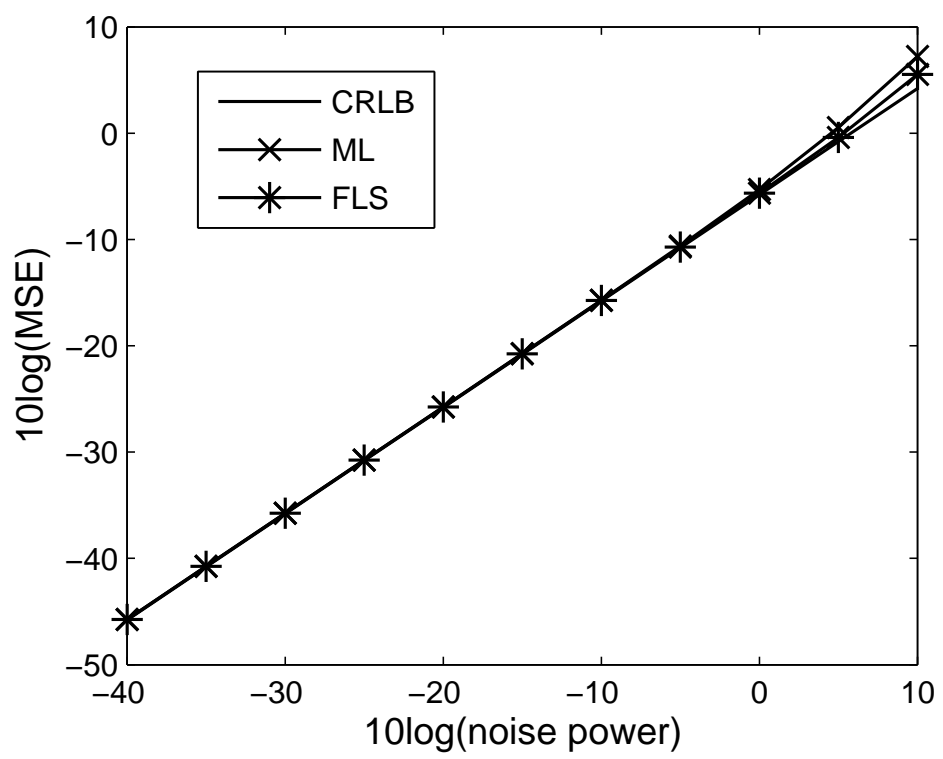

Figure 2.4: Performance comparison of the ML and FLS method when $N=20, r=10$ and $\beta=2 \pi$. 


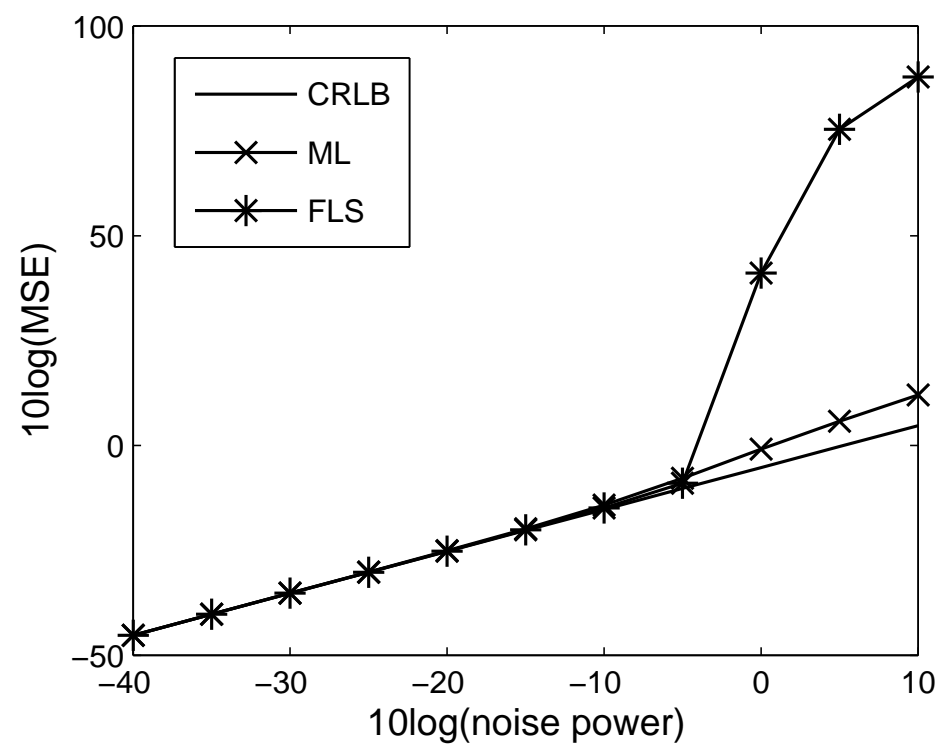

Figure 2.5: Performance comparison of the ML and FLS method when $N=20, r=2$ and $\beta=2 \pi$. 


\section{Chapter 3}

\section{Circle Fitting Using Semi-definite}

\section{Programming}

\subsection{Problem Formulation}

Let the measurement points be

$$
\mathbf{s}_{i}=\mathbf{s}_{i}^{o}+\mathbf{n}_{i}, \quad i=1,2, \cdots, M
$$

where $\mathbf{s}_{i}=\left[x_{i}, y_{i}\right]^{T}, \mathbf{s}_{i}^{o}=\left[x_{i}^{o}, y_{i}^{o}\right]^{T}$ is the true data point on a circle centered at $\mathbf{c}^{o}=\left[x^{o}, y^{o}\right]^{T}$ with radius $r^{o} . \mathbf{n}_{i}=\left[n_{x i}, n_{y i}\right]^{T}$ is the measurement noise of the $i$-th data point. It is modeled as zero mean Gaussian with covariance matrix $\mathbf{Q}_{i}$ and is independent of $\mathbf{n}_{j}$ for $j \neq i$. The relation among $\mathbf{s}_{i}^{o}, \mathbf{c}^{o}$ and $r^{o}$ is

$$
\left\|\mathbf{s}_{i}^{o}-\mathbf{c}^{o}\right\|^{2}=r^{o 2}, \quad i=1,2, \cdots, M
$$


where $\|*\|$ is the Euclidean norm.

Since $\mathbf{n}_{i} \sim N\left(\mathbf{0}, \mathbf{Q}_{i}\right)$, the ML estimator can be expressed as

$$
\begin{gathered}
\min _{\mathbf{c}^{o}, r^{o}, \mathbf{s}_{i}^{o}} \sum_{i=1}^{M}\left(\mathbf{s}_{i}-\mathbf{s}_{i}^{o}\right)^{T} \mathbf{Q}_{i}^{-1}\left(\mathbf{s}_{i}-\mathbf{s}_{i}^{o}\right) \\
\text { s.t. }\left(\mathbf{s}_{i}^{o}-\mathbf{c}^{o}\right)^{T}\left(\mathbf{s}_{i}^{o}-\mathbf{c}^{o}\right)=r^{o 2}, i=1,2, \cdots, M .
\end{gathered}
$$

The unknown parameters we intend to estimate are $\boldsymbol{\theta}^{o}=\left[\mathbf{c}^{o T}, r^{o}\right]^{T}$.

In Chapter 2 the Taylor-series linearization approach is used to obtain the ML solution through iterations. However, its estimation accuracy could be highly dependent on how it is initialized. Poor initialization may lead to a solution that converges to a local minimum instead of a global minimum. To overcome the initialization and converge problem, a ML estimator based on the SDR and SDP techniques is derived.

\subsection{SDP Solution}

In this section, we perform semi-definite relaxation on the ML estimator in order to

obtain the optimum SDP solution by utilizing the well-developed SDP solver. From (3.3), the ML estimator can be rewritten as, after dropping the irrelevant terms

$$
\begin{gathered}
\min _{\mathbf{c}^{o}, r^{o}, \mathbf{s}_{i}^{o}} \sum_{i=1}^{M}\left(\mathbf{s}_{i}^{o T} \mathbf{Q}_{i}^{-1} \mathbf{s}_{i}^{o}-2 \mathbf{s}_{i}^{T} \mathbf{Q}_{i}^{-1} \mathbf{s}_{i}^{o}\right) \\
\text { s.t. }\left(\mathbf{s}_{i}^{o}-\mathbf{c}^{o}\right)^{T}\left(\mathbf{s}_{i}^{o}-\mathbf{c}^{o}\right)=r^{o 2}, \quad i=1,2, \cdots, M .
\end{gathered}
$$


Let $\operatorname{Tr}(*)$ be the trace operation and $\mathbf{Z}_{i}=\mathbf{s}_{i}^{o} \mathbf{s}_{i}^{o T}$. Using the matrix property about the trace operation, (3.4) can be written as

$$
\begin{array}{r}
\min \sum_{i=1}^{M}\left\{\operatorname{Tr}\left(\mathbf{Q}_{i}^{-1} \mathbf{Z}_{i}\right)-2 \mathbf{s}_{i}^{T} \mathbf{Q}_{i}^{-1} \mathbf{s}_{i}^{o}\right\} \\
\text { s.t. }\left(\mathbf{s}_{i}^{o}-\mathbf{c}^{o}\right)^{T}\left(\mathbf{s}_{i}^{o}-\mathbf{c}^{o}\right)=r^{o 2}, i=1,2, \cdots, M .
\end{array}
$$

Let $\mathbf{a}_{i}^{o}=r^{o}\left[\cos \alpha_{i}, \sin \alpha_{i}\right]^{T}, i=1,2, \cdots, M$, where $\alpha_{i}$ is the angle of the $i$-th data point $\mathbf{s}_{i}^{o}$ with respect to the circle center $\mathbf{c}^{o}$. Exploring the relation between $\mathbf{s}_{i}^{o}$ and $\mathbf{a}_{i}^{o}$ yields

$$
\mathbf{s}_{i}^{o}=\mathbf{a}_{i}^{o}+\mathbf{c}^{o},\left\|\mathbf{a}_{i}^{o}\right\|^{2}=\mathbf{a}_{i}^{o T} \mathbf{a}_{i}^{o}=r^{o 2}
$$

In addition, we shall denote $\mathbf{B}_{i}=\left[\mathbf{a}_{i}^{o}, \mathbf{c}^{o}\right]$ and it is used to form the matrix $\mathbf{D}_{i}, i=$ $1,2, \cdots, M$, as

$$
\mathbf{D}_{i}=\left[\begin{array}{cc}
\mathbf{B}_{i}^{T} \mathbf{B}_{i} & \mathbf{B}_{i}^{T} \\
\mathbf{B}_{i} & \mathbf{I}_{2}
\end{array}\right]=\left[\begin{array}{ccc}
\mathbf{a}_{i}^{o T} \mathbf{a}_{i}^{o} & \mathbf{a}_{i}^{o T} \mathbf{c}^{o} & \mathbf{a}_{i}^{o T} \\
\mathbf{c}^{o T} \mathbf{a}_{i}^{o} & \mathbf{c}^{o T} \mathbf{c}^{o} & \mathbf{c}^{o T} \\
\mathbf{a}_{i}^{o} & \mathbf{c}^{o} & \mathbf{I}_{2}
\end{array}\right]
$$

where $\mathbf{I}_{2}$ is an identity matrix of size 2 . Note that the circle radius $r^{o}$ is embedded in the matrix $\mathbf{D}_{i}$. Also, let $\mathbf{1}$ be a $2 \times 1$ vector of unity, $\operatorname{Tr}\left(\mathbf{Z}_{i}\right)$ can be expressed in terms of the elements of $\mathbf{D}_{i}$ by

$$
\operatorname{Tr}\left(\mathbf{Z}_{i}\right)=\mathbf{s}_{i}^{o T} \mathbf{s}_{i}^{o}=\left(\mathbf{a}_{i}^{o}+\mathbf{c}^{o}\right)^{T}\left(\mathbf{a}_{i}^{o}+\mathbf{c}^{o}\right)=\mathbf{1}^{T} \mathbf{D}_{i}(1: 2,1: 2) \mathbf{1}
$$

Substituting (3.6), (3.7) and (3.8) into (3.5) yields the ML estimator in terms of 
unknowns $\mathbf{Z}_{i}$ and $\mathbf{D}_{i}$ :

$$
\begin{gathered}
\min _{\mathbf{Z}_{i}, \mathbf{D}_{i}} \sum_{i=1}^{M}\left\{\operatorname{Tr}\left(\mathbf{Q}_{i}^{-1} \mathbf{Z}_{i}\right)-2 \mathbf{s}_{i}^{T} \mathbf{Q}_{i}^{-1}\left[\mathbf{D}_{i}(3: 4,1: 2) \mathbf{1}\right]\right\} \\
\text { s.t. } \operatorname{Tr}\left(\mathbf{Z}_{i}\right)=\mathbf{1}^{T} \mathbf{D}_{i}(1: 2,1: 2) \mathbf{1} \\
\mathbf{Z}_{i}=\left[\mathbf{D}_{i}(3: 4,1: 2) \mathbf{1}\right]\left[\mathbf{D}_{i}(3: 4,1: 2) \mathbf{1}\right]^{T} \\
\mathbf{D}_{i}=\left[\begin{array}{cc}
\mathbf{B}_{i}^{T} \mathbf{B}_{i} & \mathbf{B}_{i}^{T} \\
\mathbf{B}_{i} & \mathbf{I}_{2}
\end{array}\right], i=1,2, \cdots, M \\
\mathbf{D}_{j}(2: 4,2)=\mathbf{D}_{1}(2: 4,2) \\
\mathbf{D}_{j}(1,1)=\mathbf{D}_{1}(1,1), \quad j=2,3, \cdots, M .
\end{gathered}
$$

Note that in (3.9) the quantity to minimize is convex with respect to $\mathbf{Z}_{i}$ and $\mathbf{D}_{i}$ but the constraints on them are not convex. We shall next apply the SDR technique to translate (3.9) from a nonconvex problem to an approximate but convex problem.

When applying SDR to the constraints in (3.9) to relax the matrix rank, (3.9) can be approximated by the following convex optimization problem

$$
\begin{aligned}
& \min _{\mathbf{Z}_{i}, \mathbf{D}_{i}} \sum_{i=1}^{M}\left\{\operatorname{Tr}\left(\mathbf{Q}_{i}^{-1} \mathbf{Z}_{i}\right)-2 \mathbf{s}_{i}^{T} \mathbf{Q}_{i}^{-1} \mathbf{D}_{i}(3: 4,1: 2) \mathbf{1}\right\} \\
& \text { s.t. } \operatorname{Tr}\left(\mathbf{Z}_{i}\right)=\mathbf{1}^{T} \mathbf{D}_{i}(1: 2,1: 2) \mathbf{1} \\
& {\left[\begin{array}{cc}
\mathbf{Z}_{i} & \mathbf{D}_{i}(3: 4,1: 2) \mathbf{1} \\
\mathbf{1}^{T} \mathbf{D}_{i}(3: 4,1: 2)^{T} & 1
\end{array}\right] \succeq \mathbf{0}_{3 \times 3}} \\
& \mathbf{D}_{i} \succeq \mathbf{0}_{4 \times 4}, \quad i=1,2, \cdots, M \\
& \mathbf{D}_{j}(2: 4,2)=\mathbf{D}_{1}(2: 4,2) \\
& \mathbf{D}_{j}(1,1)=\mathbf{D}_{1}(1,1), \quad j=2,3, \cdots, M,
\end{aligned}
$$


where the symbol $\succeq$ denotes the matrix is symmetric positive semi-definite.

Now a SDP solver can be utilized to obtain the solutions of $\mathbf{Z}_{i}$ and $\mathbf{D}_{i}, i=$ $1,2, \cdots, M$. After $\mathbf{D}_{i}$ 's are found, the estimates of the circle center and radius are obtained as

$$
\begin{aligned}
& \hat{\mathbf{c}}=\mathbf{D}_{1}(3: 4,2), \\
& \hat{r}=\frac{1}{M} \sum_{i=1}^{M}\left\|\mathbf{D}_{i}(3: 4,1)\right\| .
\end{aligned}
$$

We shall simply refer (3.10) - (3.11) as the SDP solution of circle fitting.

\subsection{Simulation}

In this section we shall examine the performance of the proposed SDP solution via simulations. The $M$ data points are collected randomly from an arc of a circle ranging from 0 to $\beta$. The circle center is $\mathbf{c}^{o}=(0,0)$ and the radius is $r^{o}=10$. Fig. 3.1 shows how the true data points are distributed when $M=5$ and $\beta=2 \pi$. The noisy measurements are generated by adding zero mean Gaussian noise to the true data points. The noise in each measurement point is I.I.D. and its covariance matrix is $\mathbf{Q}_{i}=\sigma^{2} \mathbf{I}_{2 \times 2}$. The mean square error (mse) of the parameter estimate is computed as $\operatorname{mse}(\boldsymbol{\theta})=\sum_{l=1}^{L}\left\|\boldsymbol{\theta}(l)-\boldsymbol{\theta}^{o}\right\|^{2} / L$, where $L$ is the the number of ensemble runs, $\boldsymbol{\theta}(l)$ is the solution estimate at ensemble $l$ and $\boldsymbol{\theta}^{o}$ is the true value of the circle parameters. Unless specified otherwise, $L$ is set to 500 in the simulations. The implementation of the proposed SDP solution uses the YALMIP [50] toolbox in MATLAB and the SDPT3 [51] solver.

Fig. 3.2 compares the performance of the proposed SDP solution with the Kåsa 
method when $M=5$ and $\beta=2 \pi$. The noise power varies from $10^{-4}$ to $10^{2}$ in the simulation. The upper set of results is for circle center and the lower set is for the circle radius. In each set, the straight line, the star symbols and the circle symbols are the CRLB, the mse of the SDP solution and the mse of the Kåsa method. For the circle center, when the noise power is less than 10, both methods are able to reach the CRLB accuracy. The Kåsa method starts to deviate from the CRLB when the noise power is larger than 10 while the SDP solution remains to be at the CRLB accuracy. For circle radius estimation, both methods move away from the CRLB when the noise power reaches 10 but the deviation from the SDP solution is less than the Kåsa method.

Fig. 3.3 depicts the averaged fitted circle (denoted by dash line) from the SDP solution using the same simulation configuration as in Fig. 3.2 except that the noise power is set to 2 in this case. We can observe that the circle estimated by the SDP solution is very close to the true one.

Fig. 3.4 shows the performances of the SDP solution and the Kåsa method when $M=5$ and $\beta=\pi$. Similar to the observation in Fig. 3.2, on the aspect of circle center estimation, the SDP solution can reach the CRLB accuracy when the noise power is not larger than 10. The Kåsa method begins to depart from the CRLB when the noise power reaches 10 . For the circle radius estimation, the performances of both methods become worse and worse when the noise power is equal to or larger than 1.

With $M=5$ and $\beta$ further reduced to $\pi / 3$, Fig. 3.5 gives the resultant mse of the circle center and radius together from the SDP solution and the Kåsa method. Because of the short circle segment from which the data points are sampled, the performance of both methods is affected by the bias and is not able to reach the 
CRLB accuracy after the noise power exceeds 0.1 .

To examine the effect of the number of data points $M$ on the algorithm performance, we fix the noise power to 1 and vary $M$ from 5 to 30 , where the data points are sampled randomly from the entire circle. Also we increase the number of ensemble runs to 2000. The results are shown in Fig. 3.6. The upper set of results is for the circle center whereas the lower set is for the circle radius. Performance difference between the SDP solution and the Kåsa method appears in the circle radius estimation when the total number of data points exceeds 15 . When the number of data points reaches 30 , the Kåsa method has about $1 \mathrm{~dB}$ deviation from the CRLB whereas the SDP solution is still able to provide an estimate that is quite close to the CRLB accuracy.

\subsection{Concluding Remarks}

Using the relaxation approach, this chapter proposes a semi-definite programming solution for the circle fitting problem. The ML estimator is reformulated and relaxed to arrive at an approximate but convex problem that can be solved using the welldeveloped SDP solver. Simulations are performed and the results illustrate that the performance of the proposed SDP solution is slightly better than the well-known Kåsa method and is able to reach the CRLB accuracy when the noise level is not high. Previous studies [15] and [44] have shown that the SDP algorithms have better noise resistance comparing with other algorithms. This property is not obvious from our SDP solution for circle fitting when comparing with the Kåsa method. Further investigation will be conducted in this regard. 


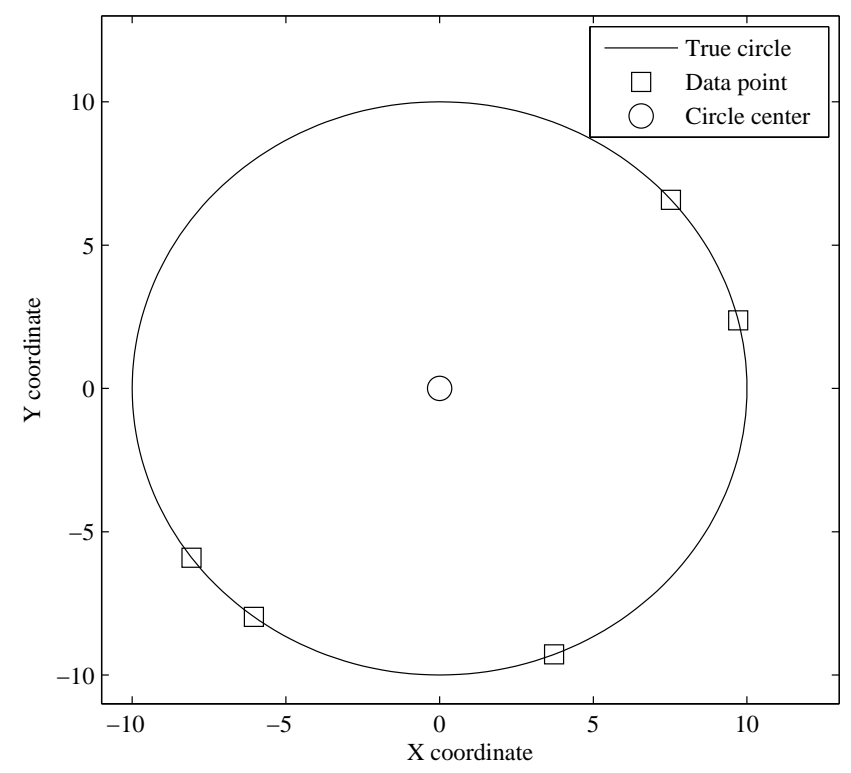

Figure 3.1: Distribution of the true data points on the circle, $M=5$ and $\beta=2 \pi$.

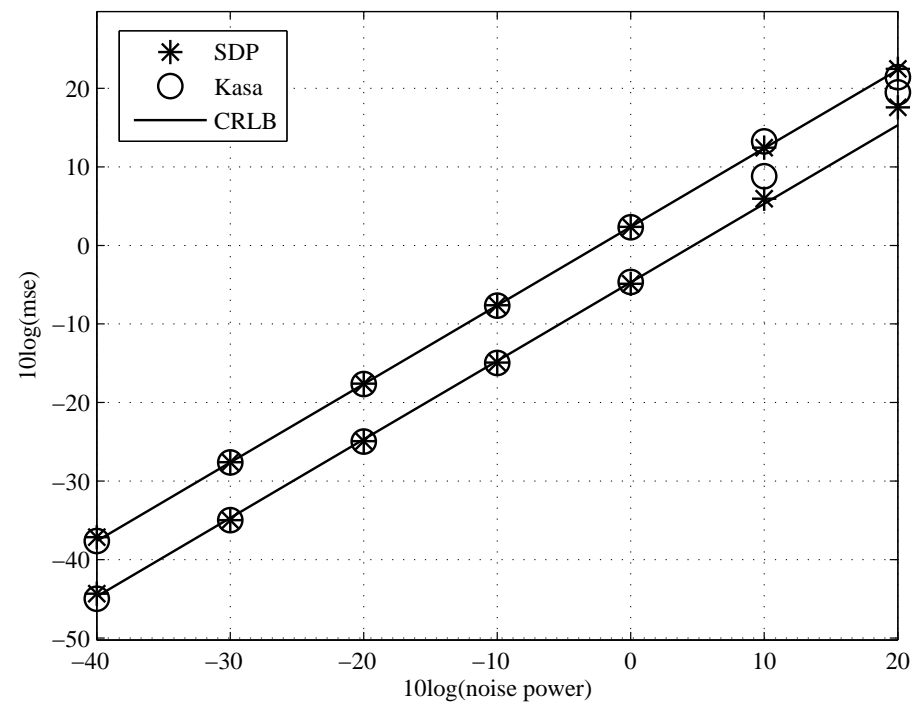

Figure 3.2: Performance comparison of the SDP and the Kåsa methods when $M=5$ and $\beta=2 \pi$. The upper set of results is for circle center and the lower set is for circle radius. 


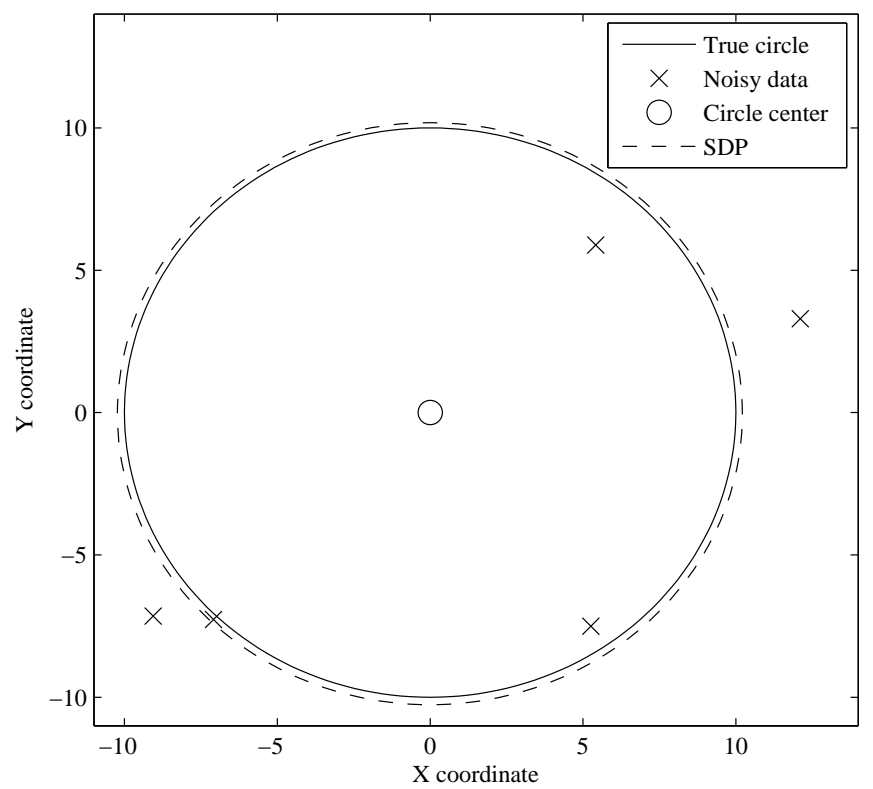

Figure 3.3: Fitted circle from the SDP solution when $M=5$ and $\beta=2 \pi$, the noise power is 2 .

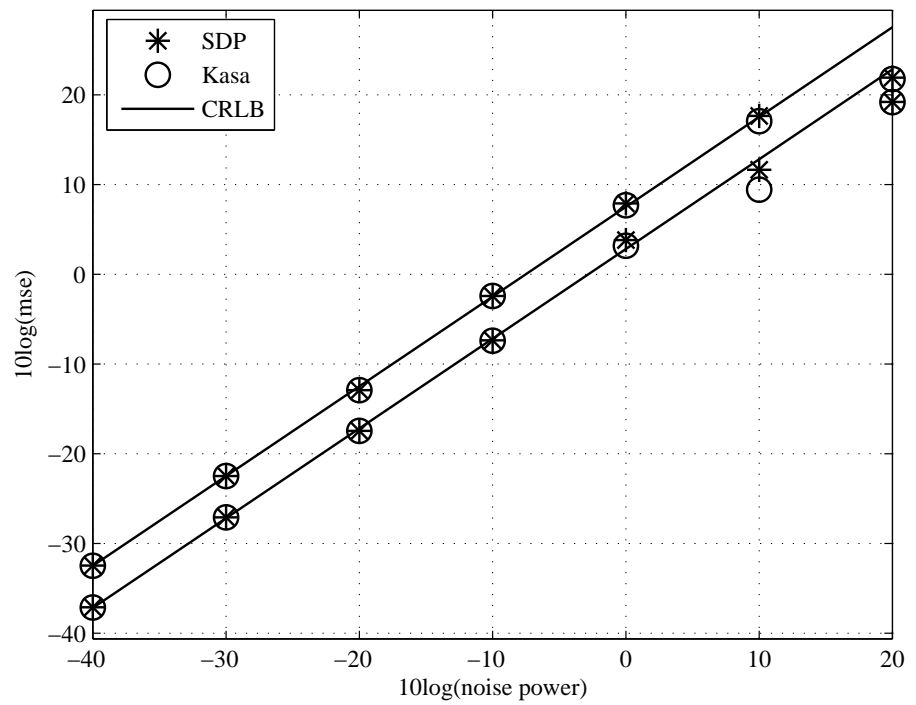

Figure 3.4: Performance comparison of the SDP and the Kåsa methods when $M=5$ and $\beta=\pi$. The upper set of results is for circle center and the lower set is for circle radius. 


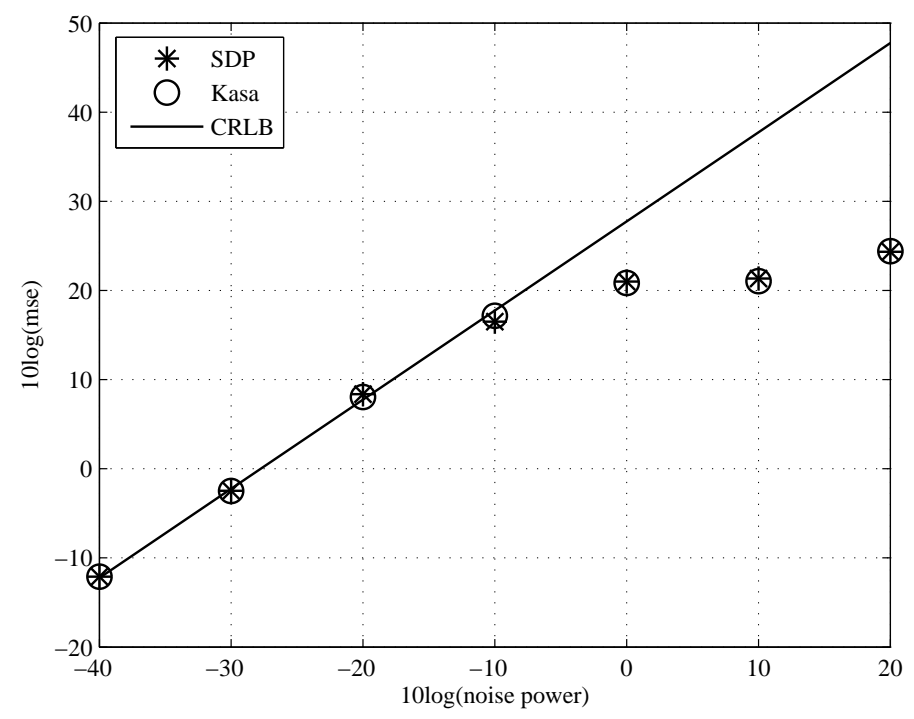

Figure 3.5: Performance comparison of the SDP and the Kåsa methods when $M=5$ and $\beta=\pi / 3$. The results are for the mses of circle center and radius together since the mse of circle center and the mse of circle radius are nearly the same.

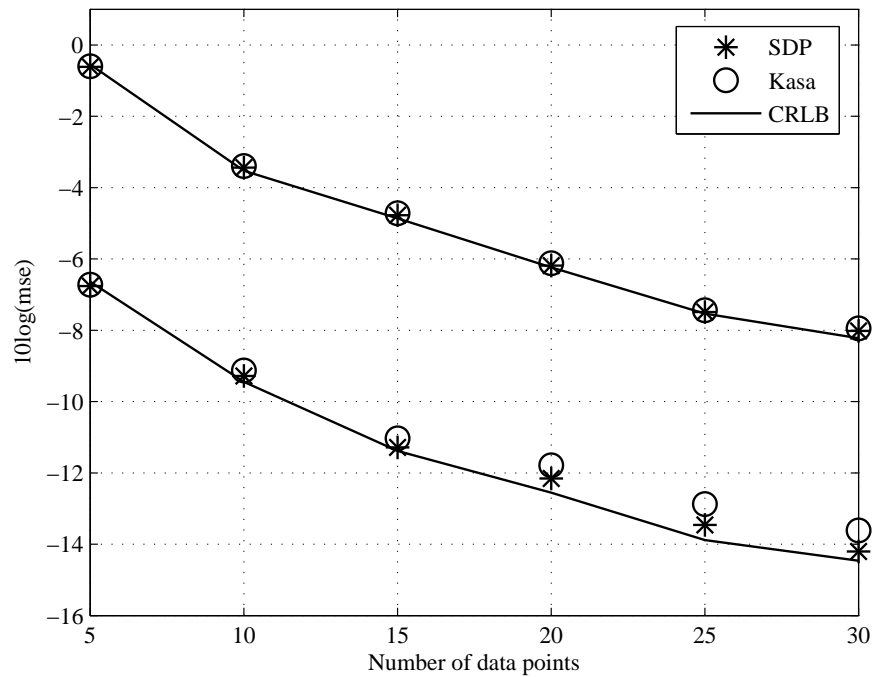

Figure 3.6: Performance comparison of the SDP and the Kåsa methods when $M$ varies, $\beta=2 \pi$ and the noise power is equal to 1 . The upper set of results is for circle center and the lower set is for circle radius. 


\section{Chapter 4}

\section{Asymptotically Efficient}

\section{Estimators for the Fitting of}

\section{Coupled Circles and Ellipses}

\subsection{Problem Formulation}

The iterative circle fitting methods presented in Chapter 2 and Chapter 3 are designed for the fitting of a single circle. Many objects encountered in practice are coupled geometric objects such as concentric circles and concentric ellipses. However, not many studies in the literature are available for the fittings of them. In this chapter asymptotically efficient estimators for the fittings of coupled circles and ellipses are derived, which can also be reduced back to the fittings of a single circle or ellipse.

For ease of illustration, we shall only consider a pair of objects that has two circles or two ellipses coupled together. The coupled geometric shapes considered here have the same center and are scaled versions of each other. Examples of such kind of 
objects include the inner and outer boundaries of a pipe and the inner and outer edges of an iris. The derived solutions can be easily extended to more than two coupled geometric shapes or reduced to one in a very direct manner.

Let $N_{i}$ be the number of data points on the $i$-th component of the coupled objects, $i=1,2$. The data from the coupled objects are modeled as

$$
\mathbf{s}_{i j}=\mathbf{s}_{i j}^{o}+\mathbf{n}_{i j}, j=1,2, \cdots, N_{i}, i=1,2,
$$

where $\mathbf{s}_{i j}=\left[\begin{array}{ll}x_{i j} & y_{i j}\end{array}\right]^{T}$ represents the $2 \times 1$ vector containing the Cartesian coordinates of a data point, $\mathbf{s}_{i j}^{o}=\left[\begin{array}{ll}x_{i j}^{o} & y_{i j}^{o}\end{array}\right]^{T}$ is the true value and $\mathbf{n}_{i j}=\left[\begin{array}{ll}n_{x_{i j}} & n_{y_{i j}}\end{array}\right]^{T}$ is the observation noise of $\mathbf{s}_{i j}$.

In the case of concentric circles, $\mathbf{s}_{i j}^{o}$ satisfies the following relation

$$
\left\|\mathbf{s}_{i j}^{o}-\mathbf{c}^{o}\right\|^{2}=r_{i}^{o 2}
$$

where $\mathbf{c}^{o}=\left[\begin{array}{ll}a^{o} & b^{o}\end{array}\right]^{T}$ is the common circle center, $r_{1}^{o}$ and $r_{2}^{o}$ are the two circle radii, and $\|*\|$ is the Euclidean norm. The unknown parameters we intend to estimate are $\boldsymbol{\theta}_{c}^{o}=\left[\begin{array}{llll}a^{o} & b^{o} & r_{1}^{o} & r_{2}^{o}\end{array}\right]^{T}$.

For concentric ellipses having the same shape and orientation as those considered in this study, $\mathbf{s}_{i j}^{o}$ fulfills the ellipse equation

$$
\begin{gathered}
\left(\mathbf{s}_{i j}^{o}-\mathbf{c}^{o}\right)^{T} \boldsymbol{\Gamma}_{i}^{o}\left(\mathbf{s}_{i j}^{o}-\mathbf{c}^{o}\right)=1, \\
\boldsymbol{\Gamma}_{1}^{o}=\left[\begin{array}{cc}
A_{1}^{o} & \rho_{1}^{o} \\
\rho_{1}^{o} & B_{1}^{o}
\end{array}\right], \boldsymbol{\Gamma}_{2}^{o}=\left[\begin{array}{ll}
A_{2}^{o} & \rho_{2}^{o} \\
\rho_{2}^{o} & B_{2}^{o}
\end{array}\right]=\varepsilon^{o} \boldsymbol{\Gamma}_{1}^{o},
\end{gathered}
$$


where $\mathbf{c}^{o}=\left[\begin{array}{ll}a^{o} & b^{o}\end{array}\right]^{T}$ is the common ellipse center, $\varepsilon^{o}$ is a positive constant scalar and $\Gamma_{i}^{o}$ defines the size, eccentricity and rotation angle of ellipse $i$. In our convention, ellipse 1 is the smaller one. The semi-major and semi-minor axes of ellipse $i$ are equal to $\left[\frac{A_{i}^{o}+B_{i}^{o} \pm \sqrt{\left(A_{i}^{o}-B_{i}^{o}\right)^{2}+4 \rho_{i}^{o}}}{2\left(A_{i}^{o} B_{i}^{o}-\rho_{i}^{o 2}\right)}\right]^{1 / 2}$ and the rotation angle with respect to the $\mathrm{x}$-axis is $\tan ^{-1}\left(\frac{-\left(A_{i}^{o}-B_{i}^{o}\right)-\sqrt{\left(A_{i}^{o}-B_{i}^{o}\right)^{2}+4 \rho_{i}^{o}}}{2 \rho_{i}^{o}}\right)$. The unknown parameters to be estimated are $\boldsymbol{\theta}_{e}^{o}$ $=\left[\begin{array}{llllll}a^{o} & b^{o} & A_{1}^{o} & \rho_{1}^{o} & B_{1}^{o} & \varepsilon^{o}\end{array}\right]^{T}$.

For notation simplicity, we shall collect all $N=N_{1}+N_{2}$ measurement data points together and represent them as

$$
\mathbf{s}=\mathbf{s}^{o}+\mathbf{n}
$$

where $\mathbf{s}=\left[\mathbf{s}_{11}^{T}, \cdots, \mathbf{s}_{1 N_{1}}^{T}, \mathbf{s}_{21}^{T}, \cdots, \mathbf{s}_{2 N_{2}}^{T}\right]^{T}$ is a $2 N \times 1$ vector, $\mathbf{s}^{o}$ is the true value of $\mathbf{s}$ and $\mathbf{n}=\left[\mathbf{n}_{11}^{T}, \cdots, \mathbf{n}_{1 N_{1}}^{T}, \mathbf{n}_{21}^{T}, \cdots, \mathbf{n}_{2 N_{2}}^{T}\right]^{T}$ is the noise vector. In this study, we shall model $\mathbf{n}$ as zero-mean Gaussian with a covariance matrix equal to $\mathbf{Q}$. In general, $\mathbf{Q}$ is not diagonal and examples are in robotics [4] and archaeology [30] applications. In most cases, it is reasonable to assume $\mathbf{n}_{i k}$ and $\mathbf{n}_{i l}$, for $k \neq l$, are independent so that $\mathbf{Q}$ is block diagonal while the elements in $\mathbf{n}_{i j}$ are correlated. It should be noted that the proposed fitting solutions and the KCR lower bound do not require $\mathbf{Q}$ to be of any particular form.

\subsection{Proposed Fitting Solutions}

In this section, we derive the proposed estimators for concentric circles and concentric ellipses based on parameter transformation and weighted equation error formulation. 


\subsubsection{Concentric Circles}

Expressing the true data point as $\mathbf{s}_{i j}^{o}=\mathbf{s}_{i j}-\mathbf{n}_{i j}$ in (4.2) gives $\left\|\mathbf{s}_{i j}-\mathbf{c}^{o}-\mathbf{n}_{i j}\right\|^{2}=r_{i}^{o 2}$. Expanding the square on the left and rearranging the terms yield

$$
2\left(\mathbf{s}_{i j}-\mathbf{c}^{o}\right)^{T} \mathbf{n}_{i j}-\mathbf{n}_{i j}^{T} \mathbf{n}_{i j}=\left\|\mathbf{s}_{i j}\right\|^{2}-2 \mathbf{s}_{i j}^{T} \mathbf{c}^{o}-\left(r_{i}^{o 2}-\left\|\mathbf{c}^{o}\right\|^{2}\right) .
$$

The left side is the equation error and the right side is its functional dependency on the concentric circles parameters. Collecting all $N$ equations for $j=1,2, \cdots, N_{i}$ and $i=1,2$ gives

$$
\mathbf{B}_{c} \mathbf{n}-\boldsymbol{\eta}=\mathbf{h}_{c}-\mathbf{G}_{c} \boldsymbol{\varphi}_{c}^{o}
$$

where

$$
\mathbf{B}_{c}=2\left[\begin{array}{cccccc}
\left(\mathbf{s}_{11}-\mathbf{c}^{o}\right)^{T} & \cdots & \mathbf{0}^{T} & \mathbf{0}^{T} & \cdots & \mathbf{0}^{T} \\
\vdots & \ddots & \vdots & \vdots & \ddots & \vdots \\
\mathbf{0}^{T} & \cdots & \left(\mathbf{s}_{1 N_{1}}-\mathbf{c}^{o}\right)^{T} & \mathbf{0}^{T} & \cdots & \mathbf{0}^{T} \\
\mathbf{0}^{T} & \cdots & \mathbf{0}^{T} & \left(\mathbf{s}_{21}-\mathbf{c}^{o}\right)^{T} & \cdots & \mathbf{0}^{T} \\
\vdots & \ddots & \vdots & \vdots & \ddots & \vdots \\
\mathbf{0}^{T} & \cdots & \mathbf{0}^{T} & \mathbf{0}^{T} & \cdots & \\
& & & & &
\end{array}\right],
$$




$$
\mathbf{h}_{c}=\left[\begin{array}{c}
\left\|\mathbf{s}_{11}\right\|^{2} \\
\vdots \\
\left\|\mathbf{s}_{1 N_{1}}\right\|^{2} \\
\left\|\mathbf{s}_{21}\right\|^{2} \\
\vdots \\
\left\|\mathbf{s}_{2 N_{2}}\right\|^{2}
\end{array}\right], \quad \mathbf{G}_{c}=\left[\begin{array}{ccc}
2 \mathbf{s}_{11}^{T} & 1 & 0 \\
\vdots & \vdots & \vdots \\
2 \mathbf{s}_{1 N_{1}}^{T} & 1 & 0 \\
2 \mathbf{s}_{21}^{T} & 0 & 1 \\
\vdots & \vdots & \vdots \\
2 \mathbf{s}_{2 N_{2}}^{T} & 0 & 1
\end{array}\right], \quad \boldsymbol{\varphi}_{c}^{o}=\left[\begin{array}{c}
\mathbf{c}^{o} \\
r_{1}^{o 2}-\left\|\mathbf{c}^{o}\right\|^{2} \\
r_{2}^{o 2}-\left\|\mathbf{c}^{o}\right\|^{2}
\end{array}\right] .
$$

In $\mathbf{B}_{c}$, $\mathbf{0}$ is a $2 \times 1$ vector of zero. $\mathbf{n}$ is defined below (4.4) and $\boldsymbol{\eta}$ is an $N \times 1$ vector whose elements are $\mathbf{n}_{i j}^{T} \mathbf{n}_{i j} . \varphi_{c}^{o}$ is the re-parameterization form of $\boldsymbol{\theta}_{c}^{o}$ and there is a unique one-to-one mapping relationship between them because $r_{i}^{o}$ is always positive. $\boldsymbol{\varphi}_{c}^{o}$ is the unknown vector to be found.

The measurement equation (4.6) is linear with respect to $\boldsymbol{\varphi}_{c}^{o}$. When the SNR is sufficient such that the second order noise components are negligible, $\mathbf{B}_{c}$ can be treated as nearly noiseless and $\boldsymbol{\eta}$ is insignificant. Since $\mathbf{n}$ is Gaussian, if we ignore the dependency of $\mathbf{B}_{c}$ with $\mathbf{c}^{o}$, the negative of the log-likelihood function corresponding to (4.6) for minimization is $\left(\mathbf{h}_{c}-\mathbf{G}_{c} \boldsymbol{\varphi}_{c}^{o}\right)^{T} \mathbf{W}_{c}\left(\mathbf{h}_{c}-\mathbf{G}_{c} \boldsymbol{\varphi}_{c}^{o}\right)$, where $\mathbf{W}_{c}$ is the weighting matrix equal to the inverse of the noise covariance [6]:

$$
\mathbf{W}_{c}=\left(\mathbf{B}_{c} \mathbf{Q B}_{c}^{T}\right)^{-1} .
$$

Other choices of the weighting matrix could be used instead, which would result in higher estimation variance and different amount of bias. Note that $\mathbf{W}_{c}$ contains the concentric circles center which is unknown to us. Some previous studies show that error in the weighting matrix does not affect the solution accuracy much [52]. 
Assuming some approximation of $\mathbf{W}_{c}$ in (4.9) is available, the solution of $\boldsymbol{\varphi}_{c}^{o}$ is

$$
\boldsymbol{\varphi}_{c}=\left(\mathbf{G}_{c}^{T} \mathbf{W}_{c} \mathbf{G}_{c}\right)^{-1} \mathbf{G}_{c}^{T} \mathbf{W}_{c} \mathbf{h}_{c} .
$$

Since $\mathbf{G}_{c}$ contains the noisy measurements $\mathbf{s}_{i}$, the ill-conditioned problem may occur, although very rarely, in performing the inverse in (4.10). In such a case, pseudo inverse should be used instead.

The solution strategy is therefore setting $\mathbf{W}_{c}$ to identity to obtain an initial estimate of $\boldsymbol{\varphi}_{c}^{o}$ (the least-squares solution), from which a better $\mathbf{W}_{c}$ can be generated to yield a better solution. The process can be iterated to improve performance. The convergence of the iterations has been studied in [53]. Through extensive simulations, we find that iterating equations (4.7)-(4.10) twice is sufficient. Further iterating to regenerate the weighting matrix is not likely to yield better results.

The proposed solution (4.10) is expected to behave similarly to the ML solution obtained directly from the data model (4.1) when the SNR is high. The transformation we use here is non-linear and introduces higher order noise components. Hence we expect the proposed solution will deviate from the actual ML solution as the SNR decreases. The proposed concentric circles estimator is translation invariant.

From the definition of $\boldsymbol{\varphi}_{c}^{o}$ in (4.8), the estimate of $\boldsymbol{\theta}_{c}^{o}$ can be found from $\boldsymbol{\varphi}_{c}$ as

$$
\boldsymbol{\theta}_{c}=\left[\begin{array}{c}
\boldsymbol{\varphi}_{c}(1) \\
\boldsymbol{\varphi}_{c}(2) \\
\sqrt{\boldsymbol{\varphi}_{c}(3)+\boldsymbol{\varphi}_{c}(1)^{2}+\boldsymbol{\varphi}_{c}(2)^{2}} \\
\sqrt{\boldsymbol{\varphi}_{c}(4)+\boldsymbol{\varphi}_{c}(1)^{2}+\boldsymbol{\varphi}_{c}(2)^{2}}
\end{array}\right]
$$




\subsubsection{Concentric Ellipses}

The proposed estimator uses the expanded form of the ellipse equation (4.3). Using $\mathbf{s}_{i j}^{o}=\mathbf{s}_{i j}-\mathbf{n}_{i j}$ and multiplying out $\mathbf{s}_{i j}^{o T} \Gamma_{i}^{o} \mathbf{s}_{i j}^{o}$ give

$$
\begin{aligned}
& 2\left(\mathbf{s}_{i j}-\mathbf{c}^{o}\right)^{T} \boldsymbol{\Gamma}_{i}^{o} \mathbf{n}_{i j}-\mathbf{n}_{i j}^{T} \boldsymbol{\Gamma}_{i}^{o} \mathbf{n}_{i j} \\
= & x_{i j}^{2} A_{i}^{o}+2 x_{i j} y_{i j} \rho_{i}^{o}+y_{i j}^{2} B_{i}^{o}-2 \mathbf{s}_{i j}^{T} \boldsymbol{\Gamma}_{i}^{o} \mathbf{c}^{o}+\mathbf{c}^{o T} \boldsymbol{\Gamma}_{i}^{o} \mathbf{c}^{o}-1 .
\end{aligned}
$$

When the noise $\mathbf{n}_{i j}$ is zero, (4.12) becomes the familiar conic equation of the form $\bar{A} x^{2}+\bar{B} x y+\bar{C} y^{2}+\bar{D} x+\bar{E} y+\bar{F}=0$ in ellipse fitting [31].

For simplicity, let

$$
k^{o}=\mathbf{c}^{o T} \boldsymbol{\Gamma}_{1}^{o} \mathbf{c}^{o}-1 .
$$

We have from ellipse 1

$$
\begin{aligned}
& 2\left(\mathbf{s}_{1 j}-\mathbf{c}^{o}\right)^{T} \boldsymbol{\Gamma}_{1}^{o} \mathbf{n}_{1 j}-\mathbf{n}_{1 j}^{T} \boldsymbol{\Gamma}_{1}^{o} \mathbf{n}_{1 j} \\
= & x_{1 j}^{2} A_{1}^{o}+2 x_{1 j} y_{1 j} \rho_{1}^{o}+y_{1 j}^{2} B_{1}^{o}-2 \mathbf{s}_{1 j}^{T} \boldsymbol{\Gamma}_{1}^{o} \mathbf{c}^{o}+k^{o} .
\end{aligned}
$$

For ellipse 2, since $\boldsymbol{\Gamma}_{1}^{o}$ and $\boldsymbol{\Gamma}_{2}^{o}$ are related by $\boldsymbol{\Gamma}_{2}^{o}=\varepsilon^{o} \boldsymbol{\Gamma}_{1}^{o}$ from (4.3), we have

$$
\begin{aligned}
& 2\left(\mathbf{s}_{2 j}-\mathbf{c}^{o}\right)^{T} \boldsymbol{\Gamma}_{1}^{o} \mathbf{n}_{2 j}-\mathbf{n}_{2 j}^{T} \boldsymbol{\Gamma}_{1}^{o} \mathbf{n}_{2 j} \\
= & x_{2 j}^{2} A_{1}^{o}+2 x_{2 j} y_{2 j} \rho_{1}^{o}+y_{2 j}^{2} B_{1}^{o}-2 \mathbf{s}_{2 j}^{T} \boldsymbol{\Gamma}_{1}^{o} \mathbf{c}^{o}+k^{o}+\frac{\varepsilon^{o}-1}{\varepsilon^{o}} .
\end{aligned}
$$

The unknowns are considered to be $A_{1}^{o}, \rho_{1}^{o}, B_{1}{ }^{o}, \Gamma_{1}{ }^{o} \mathbf{c}^{o}$ and $\frac{\varepsilon^{o}-1}{\varepsilon^{o}}$, which are reparameterizations of the elements in vector $\boldsymbol{\theta}_{e}^{o}$. Collecting all $N$ equations from (4.14) 
and (4.15) in matrix form yields

$$
\mathbf{B}_{e} \mathbf{n}-\hat{\boldsymbol{\eta}}=k^{o} \mathbf{h}_{e}-\mathbf{G}_{e} \boldsymbol{\varphi}_{e}^{o},
$$

where

$$
\mathbf{B}_{e}=2\left[\begin{array}{cccccc}
\left(\mathbf{s}_{11}-\mathbf{c}^{o}\right)^{T} \boldsymbol{\Gamma}_{1}^{o} \ldots & \mathbf{0}^{T} & \mathbf{0}^{T} & \cdots & \mathbf{0}^{T} \\
\vdots & \ddots & \vdots & \vdots & \ddots & \vdots \\
\mathbf{0}^{T} & \cdots & \left(\mathbf{s}_{1 N_{1}}-\mathbf{c}^{o}\right)^{T} \boldsymbol{\Gamma}_{1}^{o} & \mathbf{0}^{T} & \cdots & \mathbf{0}^{T} \\
\mathbf{0}^{T} & \cdots & \mathbf{0}^{T} & \left(\mathbf{s}_{21}-\mathbf{c}^{o}\right)^{T} \boldsymbol{\Gamma}_{1}^{o} \cdots & \mathbf{0}^{T} \\
\vdots & \ddots & \vdots & \vdots & \ddots & \vdots \\
\mathbf{0}^{T} & \cdots & \mathbf{0}^{T} & \mathbf{0}^{T} & \cdots\left(\mathbf{s}_{2 N_{2}}-\mathbf{c}^{o}\right)^{T} \boldsymbol{\Gamma}_{1}^{o}
\end{array}\right],
$$




$$
\begin{aligned}
\mathbf{h}_{e}=\left[\begin{array}{c}
1 \\
\vdots \\
1 \\
1 \\
\vdots \\
1
\end{array}\right]_{N \times 1}, \boldsymbol{\varphi}_{e}^{o}=\left[\begin{array}{c}
\boldsymbol{\Gamma}_{1}^{o} \mathbf{c}^{o} \\
A_{1}^{o} \\
\rho_{1}^{o} \\
B_{1}^{o} \\
\frac{\varepsilon^{o}-1}{\varepsilon^{o}}
\end{array}\right], \\
\mathbf{G}_{e}=\left[\begin{array}{cccccc}
2 x_{11} & 2 y_{11} & -x_{11}^{2} & -2 x_{11} y_{11} & -y_{11}^{2} & 0 \\
\vdots & \vdots & \vdots & \vdots & \vdots & \vdots \\
2 x_{1 N_{1}} & 2 y_{1 N_{1}} & -x_{1 N_{1}}^{2} & -2 x_{1 N_{1}} y_{1 N_{1}} & -y_{1 N_{1}}^{2} & 0 \\
2 x_{21} & 2 y_{21} & -x_{21}^{2} & -2 x_{21} y_{21} & -y_{21}^{2} & -1 \\
\vdots & \vdots & \vdots & \vdots & \vdots & \vdots \\
2 x_{2 N_{2}} & 2 y_{2 N_{2}} & -x_{2 N_{2}}^{2} & -2 x_{2 N_{2}} y_{2 N_{2}} & -y_{2 N_{2}}^{2} & -1
\end{array}\right] .
\end{aligned}
$$

In $\mathbf{B}_{e}, \mathbf{0}$ is a $2 \times 1$ vector of zero. $\mathbf{n}$ is defined below (4.4) and $\hat{\boldsymbol{\eta}}$ is an $N \times 1$ vector whose elements are $\mathbf{n}_{i j}^{T} \boldsymbol{\Gamma}_{1}{ }^{o} \mathbf{n}_{i j}$. Note that both $k^{o}$ and $\boldsymbol{\varphi}_{e}^{o}$ are related to the concentric ellipses parameter vector $\boldsymbol{\theta}_{e}^{o}$.

The measurement equation (4.16) is linear with respect to $\varphi_{e}^{o}$. By following the same argument as in the concentric circles case, the negative of the log-likelihood function corresponding to (4.16) for minimization is

$$
\left(k^{o} \mathbf{h}_{e}-\mathbf{G}_{e} \boldsymbol{\varphi}_{e}^{o}\right)^{T} \mathbf{W}_{e}\left(k^{o} \mathbf{h}_{e}-\mathbf{G}_{e} \boldsymbol{\varphi}_{e}^{o}\right),
$$

where $\mathbf{W}_{e}$ is the weighting matrix and the choice of it will be discussed later in this subsection. Note that (4.19) is in quadratic form with respect to the unknowns $\varphi_{e}^{o}$ and $k^{o}$. Minimizing (4.19) with respect to them will give the unwanted trivial solutions 
$\boldsymbol{\varphi}_{e}=\mathbf{0}$ and $k=0$. To avoid the trivial solutions, the monic constraint should be imposed in (4.19), which is described below.

\section{Monic Solution}

The monic solution rewrites the cost function (4.19) as

$$
\left(\mathbf{h}_{e}-\mathbf{G}_{e}\left(\boldsymbol{\varphi}_{e}^{o} / k^{o}\right)\right)^{T}\left(k^{o 2} \mathbf{W}_{e}\right)\left(\mathbf{h}_{e}-\mathbf{G}_{e}\left(\boldsymbol{\varphi}_{e}^{o} / k^{o}\right)\right)
$$

and solves for $\varphi_{e}^{o} / k^{o}$ only. $\mathbf{W}_{e}$ is the weighting matrix chosen to be the inverse of the noise covariance

$$
\mathbf{W}_{e}=\left(\mathbf{B}_{e} \mathbf{Q} \mathbf{B}_{e}^{T}\right)^{-1}
$$

to achieve the minimum variance of solution estimate [6]. Other choices of the weighting matrix could be used. However, the estimation variance will be higher and the amount of bias is different. Similar to the concentric circles case, $\mathbf{W}_{e}$ contains the ellipse parameters which are unknown to us. Assuming some approximation of $\mathbf{W}_{e}$ in (4.21) is available, the solution of $\varphi_{e}^{o} / k^{o}$ is

$$
\left(\boldsymbol{\varphi}_{e} / k\right)=\left(\mathbf{G}_{e}^{T} \mathbf{W}_{e} \mathbf{G}_{e}\right)^{-1} \mathbf{G}_{e}^{T} \mathbf{W}_{e} \mathbf{h}_{e}
$$

If the ill-conditioned problem occurs, pseudo inverse will be used instead. The mapping of $\varphi_{e} / k$ back to $\mathbf{c}, \boldsymbol{\Gamma}_{1}$ and $\varepsilon$ will give the concentric ellipses parameter vector estimate $\boldsymbol{\theta}_{\boldsymbol{e}}$. In particular, using $\boldsymbol{\Gamma}_{1} / k$ obtained from the third to the fifth element of $\boldsymbol{\varphi}_{e} / k$ together with the first two elements gives $\mathbf{c}$. Putting $\boldsymbol{\Gamma}_{1} / k$ and $\mathbf{c}$ into the definition of $k^{o}$ in (4.13) fixes $k$ and hence $\boldsymbol{\Gamma}_{1}$ can be determined. Using $k$ and the 
last element of $\varphi_{e} / k$ gives the estimate of $\varepsilon$.

The solution strategy is first setting $\mathbf{W}_{e}$ to identity to obtain an initial estimate of $\varphi_{e}^{o} / k^{o}$ (the least-squares solution), from which a better $\mathbf{W}_{e}$ can be generated to yield a better solution. Through extensive simulations, we find that iterating equations (4.17)-(4.22) twice is sufficient. Further iterating to regenerate the weighting matrix does not lead to much improvement in accuracy.

The proposed solution (4.22) is expected to behave similarly to the ML solution obtained directly from the data model (4.1) when the SNR is high, based on the same rationale as in the concentric circles case.

The monic solution requires $k^{o}$ to be not close to zero. To ensure this requirement, we add to the $\mathrm{x}$ and $\mathrm{y}$ coordinate points of the measurements a large constant, 500 in our case. After obtaining the monic solution we can simply subtract this constant out from the center estimate. The other parameter estimates are not affected by the translation in the data points. In Appendix A we provide a formal mathematical justification of our strategy regarding to $k^{o}$.

\subsection{KCR Lower Bound}

Kanatani [54] has derived the lower bound for the parameter estimation accuracy of an unbiased curve fitting estimator. However, most of the existing curve fitting estimators from the literature are biased. Chernov and Lesort [28] modified the lower bound from Kanatani for biased estimator and called it the KCR lower bound. The KCR lower bound applies to the fitting problem of any algebraic curve and provides the lower bound on the leading term of the mean-square parameter estimator error. 
In this section, we present the KCR lower bounds for the fittings of concentric circles and concentric ellipses that are derived based on the formulations in [28] and $[55]$.

\subsubsection{KCR Lower Bound for Concentric Circles Fitting}

For the concentric circles fitting, the unknown parameter vector is $\boldsymbol{\theta}_{c}^{o}=\left[\begin{array}{llll}a^{o} & b^{o} & r_{1}^{o} & r_{2}^{o}\end{array}\right]^{T}$. Its KCR lower bound is

$$
\operatorname{KCR}\left(\boldsymbol{\theta}_{c}\right)=\left[\mathbf{P}_{c}^{o T}\left(\mathbf{B}_{c}^{o} \mathbf{Q} B_{c}^{o T}\right)^{-1} \mathbf{P}_{c}^{o}\right]^{-1}
$$

where

$$
\mathbf{B}_{c}^{o}=2\left[\begin{array}{cccccc}
\left(\mathbf{s}_{11}^{o}-\mathbf{c}^{o}\right)^{T} & \cdots & \mathbf{0}^{T} & \mathbf{0}^{T} & \cdots & \mathbf{0}^{T} \\
\vdots & \ddots & \vdots & \vdots & \ddots & \vdots \\
\mathbf{0}^{T} & \cdots & \left(\mathbf{s}_{1 N_{1}}^{o}-\mathbf{c}^{o}\right)^{T} & \mathbf{0}^{T} & \cdots & \mathbf{0}^{T} \\
\mathbf{0}^{T} & \cdots & \mathbf{0}^{T} & \left(\mathbf{s}_{21}^{o}-\mathbf{c}^{o}\right)^{T} & \cdots & \mathbf{0}^{T} \\
\vdots & \ddots & \vdots & \vdots & \ddots & \vdots \\
\mathbf{0}^{T} & \cdots & \mathbf{0}^{T} & \mathbf{0}^{T} & \cdots & \left(\mathbf{s}_{2 N_{2}}^{o}-\mathbf{c}^{o}\right)^{T}
\end{array}\right],
$$

In $\mathbf{B}_{c}^{o}, \mathbf{0}$ is a $2 \times 1$ vector of zero. $\mathbf{Q}$ is the covariance matrix of the measurement data. 


\subsubsection{KCR Lower Bound for Concentric Ellipses Fitting}

The unknown parameter vector for the concentric ellipses fitting is $\boldsymbol{\theta}_{e}^{o}=\left[\begin{array}{llllll}a^{o} & b^{o} & A_{1}^{o} & \rho_{1}^{o} & B_{1}^{o} & \varepsilon^{o}\end{array}\right]^{T}$. The KCR lower bound of $\boldsymbol{\theta}_{e}^{o}$ is

$$
\operatorname{KCR}\left(\boldsymbol{\theta}_{e}\right)=\left[\mathbf{P}_{e}^{o T}\left(\mathbf{B}_{e}^{o} \mathbf{Q} \mathbf{B}_{e}^{o T}\right)^{-1} \mathbf{P}_{e}^{o}\right]^{-1},
$$

where

$$
\begin{aligned}
& \mathbf{B}_{e}^{o}=-2\left[\begin{array}{cccccc}
\left(\mathbf{s}_{11}^{o}-\mathbf{c}^{o}\right)^{T} \boldsymbol{\Gamma}_{1}^{o} \ldots & \mathbf{0}^{T} & \mathbf{0}^{T} & \cdots & \mathbf{0}^{T} \\
\vdots & \ddots & \vdots & \vdots & \ddots & \vdots \\
\mathbf{0}^{T} & \cdots & \left(\mathbf{s}_{1 N_{1}}^{o}-\mathbf{c}^{o}\right)^{T} \boldsymbol{\Gamma}_{1}^{o} & \mathbf{0}^{T} & \cdots & \mathbf{0}^{T} \\
\mathbf{0}^{T} & \cdots & \mathbf{0}^{T} & \left(\mathbf{s}_{21}^{o}-\mathbf{c}^{o}\right)^{T} \boldsymbol{\Gamma}_{1}^{o} \ldots & \mathbf{0}^{T} \\
\vdots & \ddots & \vdots & \vdots & \ddots & \vdots \\
\mathbf{0}^{T} & \cdots & \mathbf{0}^{T} & \mathbf{0}^{T} & \cdots\left(\mathbf{s}_{2 N_{2}}^{o}-\mathbf{c}^{o}\right)^{T} \boldsymbol{\Gamma}_{1}^{o}
\end{array}\right], \\
& \mathbf{P}_{e}^{o}=2\left[\begin{array}{llllll}
\mathbf{p}_{e, 11}^{o} & \cdots & \mathbf{p}_{e, 1 N_{1}}^{o} & \mathbf{p}_{e, 21}^{o} & \cdots & \mathbf{p}_{e, 2 N_{2}}^{o}
\end{array}\right]^{T}, \\
& \mathbf{p}_{e, 1 j}^{o}=\left[\begin{array}{lll}
-\left(\mathbf{s}_{1 j}^{o}-\mathbf{c}^{o}\right)^{T} \boldsymbol{\Gamma}_{1}^{o} & \mathbf{q}_{1 j}^{T} & 0
\end{array}\right]^{T}, j=1,2, \cdots, N_{1}, \\
& \mathbf{p}_{e, 2 j}^{o}=\left[\begin{array}{lll}
-\left(\mathbf{s}_{2 j}^{o}-\mathbf{c}^{o}\right)^{T} \boldsymbol{\Gamma}_{1}^{o} & \mathbf{q}_{2 j}^{T} & \frac{1}{\varepsilon^{o 2}}
\end{array}\right]^{T}, j=1,2, \cdots, N_{2}, \\
& \mathbf{q}_{i j}=\left[\begin{array}{lll}
\frac{1}{2}\left(x_{i j}^{o}-a^{o}\right)^{2} & \left(x_{i j}^{o}-a^{o}\right)\left(y_{i j}^{o}-b^{o}\right) \frac{1}{2}\left(y_{i j}^{o}-b^{o}\right)^{2}
\end{array}\right]^{T} .
\end{aligned}
$$

In $\mathbf{B}_{e}^{o}, \mathbf{0}$ is a $2 \times 1$ vector of zero. $\mathbf{Q}$ is the covariance matrix of the measurement data. 


\subsection{Performance Analysis}

In this section we perform the first order analysis of the proposed solution by evaluating its covariance matrix. The first order analysis is valid when the measurement noise is small. After obtaining the covariance matrix, we shall compare it with the KCR lower bound.

\subsubsection{Concentric Circles}

The relation between $\boldsymbol{\theta}_{c}$ and $\boldsymbol{\varphi}_{c}$ is given in (4.11). Taking the differential on both sides of (4.11) with respect to the true values gives

$$
\Delta \boldsymbol{\theta}_{c}=\boldsymbol{\Phi}_{c}^{o} \Delta \boldsymbol{\varphi}_{c}, \quad \boldsymbol{\Phi}_{c}^{o}=\left[\begin{array}{cccc}
1 & 0 & 0 & 0 \\
0 & 1 & 0 & 0 \\
\frac{a^{o}}{r_{1}^{o}} & \frac{b^{o}}{r_{1}^{o}} & \frac{1}{2 r_{1}^{o}} & 0 \\
\frac{a^{o}}{r_{2}^{o}} & \frac{b^{o}}{r_{2}^{o}} & 0 & \frac{1}{2 r_{2}^{o}}
\end{array}\right]
$$

The estimate $\boldsymbol{\theta}_{c}$ from (4.11) has negligible bias under the first order approximation and its covariance matrix is

$$
\operatorname{cov}\left(\boldsymbol{\theta}_{c}\right)=\boldsymbol{\Phi}_{c}^{o} \operatorname{cov}\left(\boldsymbol{\varphi}_{c}\right) \boldsymbol{\Phi}_{c}^{o T}
$$

From the theory of Weighted-Least-Squares (WLS) minimization [6], we have $\operatorname{cov}\left(\boldsymbol{\varphi}_{c}\right) \simeq$ $\left(\mathbf{G}_{c}^{o T} \mathbf{W}_{c}^{o} \mathbf{G}_{c}^{o}\right)^{-1}$ when the noise is small, where $\mathbf{G}_{c}^{o}$ and $\mathbf{W}_{c}^{o}$ are $\mathbf{G}_{c}$ in (4.8) and $\mathbf{W}_{c}$ in (4.9) when replacing the noisy values by the true values. Hence the covariance matrix 
of $\boldsymbol{\theta}_{c}$ for the proposed method is

$$
\operatorname{cov}\left(\boldsymbol{\theta}_{c}\right) \simeq\left[\boldsymbol{\Phi}_{c}^{o-T} \mathbf{G}_{c}^{o T} \mathbf{W}_{c}^{o} \mathbf{G}_{c}^{o} \boldsymbol{\Phi}_{c}^{o-1}\right]^{-1} .
$$

We shall next compare $\operatorname{cov}\left(\boldsymbol{\theta}_{c}\right)$ with the KCR lower bound which is given in (4.23) and has the same structural form as $\operatorname{cov}\left(\boldsymbol{\theta}_{c}\right)$ in (4.31). Using the definitions of $\mathbf{P}_{c}^{o}$ in (4.25) and $\boldsymbol{\Phi}_{c}^{o}$ in (4.29), we can verify that $\mathbf{P}_{c}^{o} \boldsymbol{\Phi}_{c}^{o}=-\mathbf{G}_{c}^{o}$ and hence

$$
\mathbf{G}_{c}^{o} \Phi_{c}^{o-1}=-\mathbf{P}_{c}^{o}
$$

Under the first order analysis, (4.9) can be approximated as

$$
\mathbf{W}_{c} \simeq\left(\mathbf{B}_{c}^{o} \mathbf{Q B}_{c}^{o T}\right)^{-1}
$$

where $\mathbf{B}_{c}^{o}$ is defined in (4.24). Putting (4.32) and (4.33) into (4.31) yields

$$
\operatorname{cov}\left(\boldsymbol{\theta}_{c}\right) \simeq\left[\mathbf{P}_{c}^{o T}\left(\mathbf{B}_{c}^{o} \mathbf{Q B} B_{c}^{o T}\right)^{-1} \mathbf{P}_{c}^{o}\right]^{-1}=\operatorname{KCR}\left(\boldsymbol{\theta}_{c}\right)
$$

Thus the proposed concentric circles estimator reaches the KCR lower bound accuracy under the first order analysis.

\subsubsection{Concentric Ellipses}

We shall perform the analysis of the proposed estimator with the monic constraint. For simplicity we shall denote $\boldsymbol{\varphi}_{e} / k$ as $\hat{\boldsymbol{\varphi}}_{e}$ and $\boldsymbol{\Gamma}_{1} / k$ as $\hat{\boldsymbol{\Gamma}}_{1}$. The monic solution $\hat{\boldsymbol{\varphi}}_{e}$ is given in (4.22). From the definition of $\varphi_{e}^{o}$ in (4.18), the differentials of the first two 
elements of $\hat{\varphi}_{e}$ are

$$
\Delta \hat{\varphi}_{e}(1: 2)=\hat{\boldsymbol{\Gamma}}_{1}^{o} \Delta \mathbf{c}+\Delta \hat{\boldsymbol{\Gamma}}_{1} \mathbf{c}^{o}
$$

$\hat{\boldsymbol{\Gamma}}_{1}$ is given by the third to the fifth elements of $\hat{\boldsymbol{\varphi}}_{e}$, whose error $\Delta \hat{\boldsymbol{\Gamma}}_{1}$ can be expressed in terms of $\Delta \hat{\boldsymbol{\varphi}}_{e}(3: 5)$. Accordingly, we have

$$
\Delta \boldsymbol{\theta}_{e}(1: 2)=\Delta \mathbf{c}=\mathbf{V}_{a b}^{T} \Delta \hat{\boldsymbol{\varphi}}_{e}
$$

where

$$
\mathbf{V}_{a b}=\left\{k^{o} \boldsymbol{\Gamma}_{1}^{o-1}\left[\begin{array}{ccccc}
1 & 0 & -\mathbf{c}^{o T} & 0 & 0 \\
0 & 1 & 0 & -\mathbf{c}^{o T} & 0
\end{array}\right]\right\}^{T}
$$

and $\hat{\boldsymbol{\Gamma}}_{1}^{o}=\boldsymbol{\Gamma}_{1}^{o} / k^{o}$ has been used. The subscript $a b$ of $\mathbf{V}_{a b}$ represents that it is for the concentric ellipses center.

The value $k$ is equal to $\left[\mathbf{c}^{T} \hat{\boldsymbol{\varphi}}_{e}(1: 2)-1\right]^{-1}$ from (4.13) and its differential is $\Delta k=-k^{o 2}\left[\mathbf{c}^{o T} \Delta \hat{\boldsymbol{\varphi}}_{e}(1: 2)+\hat{\boldsymbol{\varphi}}_{e}^{o}(1: 2)^{T} \Delta \mathbf{c}\right]$. Using $\hat{\boldsymbol{\varphi}}_{e}^{o}(1: 2)=\hat{\boldsymbol{\Gamma}}_{1}^{o} \mathbf{c}^{o}=\boldsymbol{\Gamma}_{1}^{o} \mathbf{c}^{o} / k^{o}$ from (4.18) and $\Delta \mathbf{c}$ from (4.36), we have

$$
\Delta k=\mathbf{v}_{k}^{T} \Delta \hat{\boldsymbol{\varphi}}_{e}
$$

where

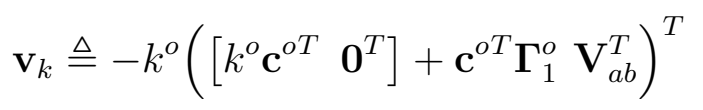

and $\mathbf{0}$ is a $4 \times 1$ vector of zero. Since $\boldsymbol{\theta}_{e}(3: 5)=k \hat{\boldsymbol{\varphi}}_{e}(3: 5)$ according to (4.18), their differentials are

$$
\Delta \boldsymbol{\theta}_{e}(3: 5)=k^{o} \Delta \hat{\boldsymbol{\varphi}}_{e}(3: 5)+\hat{\boldsymbol{\varphi}}_{e}^{o}(3: 5) \Delta k=\mathbf{V}_{\Gamma}^{T} \Delta \hat{\boldsymbol{\varphi}}_{e}
$$


where

$$
\mathbf{V}_{\Gamma}=\left(\left[\begin{array}{lll}
\mathbf{O} & k^{o} \mathbf{I} & \mathbf{0}
\end{array}\right]+\hat{\boldsymbol{\varphi}}_{e}^{o}(3: 5) \mathbf{v}_{k}^{T}\right)^{T}
$$

$\mathbf{O}$ is a $3 \times 2$ matrix of zero, $\mathbf{I}$ is the identity matrix of size $3, \mathbf{0}$ is a $3 \times 1$ vector of zero and (4.38) has been used.

The value of $\varepsilon$ is $\left[1-k \hat{\boldsymbol{\varphi}}_{e}\right]^{-1}$ from (4.18). Its differential is $\varepsilon^{o 2}\left[k^{o} \Delta \hat{\boldsymbol{\varphi}}_{e}(6)+\right.$ $\left.\Delta k \hat{\varphi}_{e}^{o}(6)\right]$ and by using (4.38) we arrive at

$$
\Delta \boldsymbol{\theta}_{e}(6)=\mathbf{v}_{\varepsilon}^{T} \Delta \hat{\boldsymbol{\varphi}}_{e}
$$

where

$$
\mathbf{v}_{\varepsilon}=\varepsilon^{o 2}\left(\left[\begin{array}{ll}
\mathbf{0}^{T} & k^{o}
\end{array}\right]+\frac{\varepsilon^{o}-1}{\varepsilon^{o} k^{o}} \mathbf{v}_{k}^{T}\right)^{T}
$$

and $\mathbf{0}$ is a $5 \times 1$ vector of zero. Thus from (4.36), (4.40) and (4.42), we obtain the relationship between the errors in $\boldsymbol{\theta}_{e}$ and $\hat{\boldsymbol{\varphi}}_{e}$ as

$$
\Delta \boldsymbol{\theta}_{e}=\boldsymbol{\Phi}_{e}^{o} \Delta \hat{\boldsymbol{\varphi}}_{e}, \boldsymbol{\Phi}_{e}^{o}=\left[\begin{array}{lll}
\mathbf{V}_{a b} & \mathbf{V}_{\Gamma} & \mathbf{V}_{\varepsilon}
\end{array}\right]^{T}
$$

Under the first order approximation, the covariance matrix of $\boldsymbol{\theta}_{e}$ is

$$
\operatorname{cov}\left(\boldsymbol{\theta}_{e}\right)=\boldsymbol{\Phi}_{e}^{o} \operatorname{cov}\left(\hat{\boldsymbol{\varphi}}_{e}\right) \boldsymbol{\Phi}_{e}^{o T}
$$

When the noise in $\mathbf{G}_{e}$ and $\mathbf{B}_{e}$ are small enough to be neglected, the covariance matrix of $\hat{\varphi}_{e}$ from WLS minimization [6] is

$$
\operatorname{cov}\left(\hat{\boldsymbol{\varphi}}_{e}\right) \simeq\left(\mathbf{G}_{e}^{o T} \mathbf{W}_{e}^{o} \mathbf{G}_{e}^{o}\right)^{-1}
$$


where $\mathbf{G}_{e}^{o}$ and $\mathbf{W}_{e}^{o}$ are $\mathbf{G}_{e}$ in (4.18) and $\mathbf{W}_{e}$ in (4.21) when replacing the noisy quantities by their true values. Since $\boldsymbol{\Phi}_{e}^{o}$ is invertible, substituting (4.46) into (4.45) yields the covariance matrix of $\boldsymbol{\theta}_{e}$ as

$$
\operatorname{cov}\left(\boldsymbol{\theta}_{e}\right)=\left(\boldsymbol{\Phi}_{e}^{o-T} \mathbf{G}_{e}^{o T} \mathbf{W}_{e}^{o} \mathbf{G}_{e}^{o} \boldsymbol{\Phi}_{e}^{o-1}\right)^{-1}
$$

The KCR lower bound for the concentric ellipses parameters is given in (4.26), which has the same structural form as $\operatorname{cov}\left(\boldsymbol{\theta}_{e}\right)$ in (4.47).

From (4.28) and (4.44), performing direct matrix multiplication and using the ellipse equation (4.3) for simplification give $\mathbf{P}_{e}^{o} \boldsymbol{\Phi}_{e}^{o}=-\mathbf{G}_{e}^{o}$. Hence

$$
\mathbf{G}_{e}^{o} \Phi_{e}^{o-1}=-\mathbf{P}_{e}^{o}
$$

Also, $\mathbf{W}_{e}$ in $(4.21)$ can be approximated as

$$
\mathbf{W}_{e} \simeq\left(\mathbf{B}_{e}^{o} \mathbf{Q} \mathbf{B}_{e}^{o T}\right)^{-1}
$$

under the first order approximation where the noise level is low. The substitution of (4.48) and (4.49) into (4.47) gives immediately

$$
\operatorname{cov}\left(\boldsymbol{\theta}_{e}\right) \simeq\left[\mathbf{P}_{e}^{o T}\left(\mathbf{B}_{e}^{o} \mathbf{Q} \mathbf{B}_{e}^{o T}\right)^{-1} \mathbf{P}_{e}^{o}\right]^{-1}=\operatorname{KCR}\left(\boldsymbol{\theta}_{e}\right)
$$

In other words, the proposed concentric ellipses fitting method achieves the KCR lower bound performance asymptotically. 


\subsection{Simulation and Experiment}

We shall examine the performance of the proposed estimators and support the theoretical developments using synthetic data and actual image captures. Their results will be presented separately.

\subsubsection{Synthetic Data}

Unless specified otherwise, the $N=N_{1}+N_{2}$ data points are collected randomly (with a uniform distribution) along partial segments of the coupled circles or coupled ellipses. The noisy measurements are generated by adding zero-mean Gaussian noise to the true data points. The noise covariance matrix $\mathbf{Q}$ is $\operatorname{diag}\{\mathbf{R}, \mathbf{R}, \cdots, \mathbf{R}\}$, where $\mathbf{R}$ is a $2 \times 2$ matrix equal to $\sigma^{2}\left[\begin{array}{ll}1 & \delta \\ \delta & 1\end{array}\right]$ and $\delta$ is the correlation coefficient in the $\mathbf{x}$ and y noise components. In most curve fitting studies, using a scaled identity matrix for $\mathbf{Q}$ is sufficient $[28,54]$. However, in application such as archaeology, the elements in $\mathbf{n}_{i j}$ for each data point can be correlated due to the process of digitizing [30]. In practice, the noise power $\sigma^{2}$ and the noise correlation factor $\delta$ can be estimated from data measurements [30]. The estimation accuracy is presented using the meansquare error (mse) of the estimate of the unknown vector $\boldsymbol{\theta}$, which is computed as $\operatorname{mse}(\boldsymbol{\theta})=\sum_{l=1}^{L}\left\|\boldsymbol{\theta}(l)-\boldsymbol{\theta}^{o}\right\|^{2} / L$, where $\boldsymbol{\theta}(l)$ is the solution obtained at ensemble run $l, \boldsymbol{\theta}^{\circ}$ is the true value and $L$ is the number of ensemble runs. $L$ is equal to 10000 throughout the simulations unless stated otherwise. The results for the concentric circles and the concentric ellipses will be shown separately in sequel, as well as the ones of the single circle and the single ellipse. 


\section{Concentric Circles and Single Circle}

The common center of the concentric circles is $\mathbf{c}^{o}=\left[\begin{array}{ll}0 & 0\end{array}\right]^{T}$ and the two radii are $r_{1}^{o}=10$ and $r_{2}^{o}=20$. In addition to the proposed estimator, the estimation results from the method proposed by O'Leary et al. [10], the Kåsa method [23, 24] and the Pratt method [25] are also shown for comparison purpose. When applying the Kåsa method on the concentric circles, the parameters of the two circles are obtained independently and the common circle center estimate is computed as the average of the two independent circle center estimates.

Fig. 4.1 and Fig. 4.2 compare the performance of the proposed estimator $(\times$ symbol) with the O'Leary method $(\bigcirc$ symbol) and the Kåsa method (+ symbol) for the concentric circles center and radii estimates. We randomly sampled 10 data points from each of the two arcs of the concentric circles starting from 0 to $\pi \mathrm{rad}$, i.e. $N_{1}=N_{2}=10$. The noise power $\sigma^{2}$ varies from $10^{-4}$ to 10 and the correlation coefficient $\delta$ is 0.8 . Fig. 4.1 shows that the proposed estimator reaches the KCR lower bound accuracy for the concentric circles center estimate very well as expected from the theory. The O'Leary and the Kåsa methods have constant differences of about 2.7 decibel $(\mathrm{dB})$ and $1.5 \mathrm{~dB}$ with the KCR lower bound before the noise power reaches 1, above which larger deviations occur. For the radii estimates shown in Fig. 4.2, the observations are similar in which the proposed method attains the KCR lower bound accuracy until the noise power exceeds 0.1 while the O'Leary and the Kåsa methods always deviate away from the bound.

To investigate a little further, we compared the estimation bias when the weighting matrix $\mathbf{W}_{c}$ is equal to (4.9) and when it is set to be an identity matrix for the simulation case corresponding to Figs. 4.1 and 4.2. The results are tabulated in 
Table 4.1 when the noise level is fixed at $10^{-4}$. It clearly shows the effect of weighting matrix on the bias. For concentric circles, when $\mathbf{W}_{c}$ is equal to (4.9) the bias is about $0.5 \mathrm{~dB}$ larger than the one if $\mathbf{W}_{c}$ is equal to an identity matrix. However, choosing $\mathbf{W}_{c}$ as in (4.9) did provide smaller estimation variance. It is non-trivial to analyze how the weighting matrix would affect the estimate bias theoretically. We plan to look at it in more detail and report the findings elsewhere.

The proposed concentric circles estimator can also generate a non-iterative solution by setting $\mathbf{W}_{c}$ in (4.10) to an identity matrix. In such a case, its performance would be suboptimal. Fig. 4.3 and Fig. 4.4 show the results of the non-iterative solution together with those from the O'Leary and Kåsa methods. From the plots we can observe that the performance of the non-iterative solution is similar to the one from the O'Leary method for both the concentric circles center and radii estimates. It is slightly worse than the Kåsa method for the center estimate but yields better results for the radii estimates. However, the proposed solution in non-iterative form is more computational efficient than the O'Leary and Kåsa methods. Table 4.2 shows the relative computation time in matlab over 10000 ensemble runs when the noise power is $10^{-4}$. The non-iterative solution requires less computation time than the other two methods. The iterative solution of the proposed estimator requires more computation time than the non-iterative version but provides much better estimation performance.

The proposed estimator can reduce back for the fitting of a single circle. By using the same setting as in Figs. 4.1 and 4.2, Figs. 4.5 and 4.6 examine the performance of the proposed estimator when the data points are only from the arc of the circle whose radius is 10 . The proposed method is able to attain the KCR lower bound accuracy for both the single circle center and radius estimates when $\sigma^{2}$ is less than 
$10^{-0.5}$ while the Kåsa and Pratt methods are not able to reach the KCR lower bound performance at all.

To supplement Figs. 4.3 - 4.6, we show in Table 4.3 numerically the performance difference with the KCR lower bounds of different estimators. The values given are obtained by averaging over the range of noise power varying from $10^{-4}$ to 0.1 .

\section{Concentric Ellipses and Single Ellipse}

The concentric ellipses center is $\mathbf{c}^{o}=\left[\begin{array}{ll}0 & 0\end{array}\right]^{T}$, the semi-major and semi-minor axes of the inner ellipse (ellipse 1) are 20 and 6.67, the rotation angle is $\pi / 6 \mathrm{rad}\left(A_{1}^{o}=\right.$ $\left.0.0075, B_{1}^{o}=0.0175, \rho_{1}^{o}=-0.0087\right)$. The scale factor between $\Gamma_{1}^{o}$ and $\Gamma_{2}^{o}$ as defined in $(4.3)$ is $\varepsilon^{o}=0.25$.

The performance of the O'Leary method [10], the Taubin method [31], the FNS method [34] and the HEIV method [35] are also evaluated for comparison. Note that except the O'Leary method, these methods are originally developed for the single ellipse fitting based on the conic ellipse equations and we have extended them for coupled ellipses. We convert their conic parameter estimates to ellipse parameters as defined in (4.3) when performing the comparison.

Fig. 4.7 gives the result of the concentric ellipses center estimate as $\sigma^{2}$ increases from $10^{-4}$ to 1 , when the noise correlation coefficient $\delta$ is -0.6 and $N_{1}=N_{2}=10$ data points are sampled randomly from the concentric ellipses portions that are above the common major axis. The proposed estimator $(\times$ symbol $)$, the FNS method $(\triangle$ symbol) and the HEIV method ( $\nabla$ symbol) attain the KCR lower bound accuracy very well when $\sigma^{2}$ is not larger than $10^{-2.5}$. After that, the FNS method deviates from the bound. When $\sigma^{2}$ reaches 0.1 , only the proposed method attains the KCR 
lower bound accuracy.

With the same settings as in Fig. 4.7, Fig. 4.8 shows the results of the estimates $\Gamma_{1}\left(A_{1}, \rho_{1}\right.$ and $\left.B_{1}\right)$ and $\varepsilon$. The upper set of results is for $\varepsilon$ and the lower one is for $\boldsymbol{\Gamma}_{1}$. The estimation accuracy of the proposed estimator and that of the HEIV method achieve the KCR lower bound when $\sigma^{2}$ is not larger than $10^{-1.5}$. We can observe from Figs. 4.7 and 4.8 the proposed method has a higher noise tolerance of the thresholding effect than the FNS and HEIV methods.

We compare the estimation bias when the weighting matrix $\mathbf{W}_{e}$ is equal to (4.21) and when it is set to an identity matrix for the simulation case corresponding to Figs. 4.7 and 4.8, and the results are tabulated in Table 4.1 at the noise level $10^{-4}$. The resulting bias and variance when $\mathbf{W}_{e}$ is chosen as (4.21) are both smaller than the ones if $\mathbf{W}_{e}$ is equal to an identity matrix. It is non-trivial to analyze how the weighting matrix would affect the estimate bias theoretically. We plan to look at it in more detail and report the findings elsewhere.

A non-iterative solution can be generated from the proposed concentric ellipses estimator by replacing $\mathbf{W}_{e}$ in (4.22) with an identity matrix. Using the same settings as in Figs. 4.7 and 4.8, Figs. 4.9 and 4.10 give the estimation performance of the non-iterative solution $(\times$ symbol $)$, the O'Leary method $(\bigcirc$ symbol $)$ and the Taubin method ( $\triangle$ symbol). When $\sigma^{2}$ is not larger than $10^{-2.5}$, the non-iterative solution has a similar performance as the other two methods. However, Table 4.2 indicates that the non-iterative solution is more computation efficient than the others. The relative computation time are obtained from matlab over 10000 ensemble runs at the noise level of $10^{-4}$. The iterative solution of the proposed estimator requires more computation time than the non-iterative version but provides much better accuracy. 
The proposed estimator can be simplified for the single ellipse fitting. Figs. 4.11 and 4.12 examine the performance of the proposed estimator using the same set of data sampled from the inner ellipse as in Figs. 4.7 and 4.8. The proposed estimator attains the KCR lower bound accuracy before $\sigma^{2}$ reaches 0.01 , while the Taubin and Fitzgibbon methods are not able to reach the bound at all. There is significant bias in the center estimate of the Fitzgibbon method when $\sigma^{2}>0.01$, making its mse lower than the KCR lower bound. The performance degradation of the proposed estimator when $\sigma^{2}$ is larger than 0.01 is caused by the thresholding effect in non-linear estimation.

\subsubsection{Experiment with Real Images}

We shall apply the proposed estimators on several real images to test their performance. The real images we use here are either taken by a digital camera or from the iris database CASIA-IrisV3 [56] collected by the Chinese Academy of Sciences' Institute of Automation (CASIA).

\section{Concentric Objects Images}

The left parts of Fig. 4.13 and Fig. 4.16 depict images of the concentric circles and concentric ellipses captured by a digital camera with a $640 \times 480$ resolution. The images are clean and have been converted to 8bpp gray scale with each pixel value between 20 and 224. To examine the estimators' performance under noise, we normalize the gray scale images by 255, add zero-mean Gaussian white noise of certain power $\xi^{2}$, truncate each pixel value to be within 0 to 1 and multiply by 255 to restore the original dynamic range. The Canny edge detection [57] is applied to the 
noisy images to obtain the edges of the concentric circles and concentric ellipses. The edges are converted to binary images as shown on the right parts of Fig. 4.13 and Fig. 4.16. The binary images define the data points on which the proposed estimators are applied.

Using the data points from concentric circles edges in Fig. 4.13, Fig. 4.14 and Fig. 4.15 examine the performance of the proposed estimator $(\times$ symbol $)$ and compare it with the O'Leary method $(\bigcirc$ symbol). The noise covariance $\mathbf{Q}$ is a scaled identity matrix in (4.9) for the proposed method. $N_{1}=N_{2}=20$ data points are randomly sampled from each of the concentric circles edges. The power $\xi^{2}$ of the zero-mean Gaussian white noise added to the original image before edge detection is varied from $10^{-4}$ to $10^{-1.4}$ and $L=5000$ ensemble runs are performed at each noise power level. Instead of the mse, the variances of the parameter estimates are shown for the performance comparison because the true parameter values are not known. Fig. 4.14 indicates the variance of the center estimate from the proposed method is lower than that from the O'Leary method. The difference between them is about $2 \mathrm{~dB}$ when the noise power is not larger than $10^{-1.8}$. This amount of performance improvement is consistent with the mse results we have in Fig. 4.1. Note that the edge detection is in terms of pixel coordinates in integers and the noise in the data is quantized to integer values. As a result, when the noise power varies from $10^{-4}$ to $10^{-1.8}$, the parameter estimation variance only changes slightly, and when the noise power reaches higher values it increases sharply. We observe from Fig. 4.15 the proposed method has only slight performance improvement on the radii when the noise power is larger than $10^{-3}$, much less than those shown in Fig. 4.2. It is because the Gaussian noise used in this experiment is white $(\delta=0)$ while the one in Fig. 4.2 is anisotropic with a 
correlation coefficient $\delta=0.8$. The improvement in radii estimates increases when $\delta$ increases.

Fig. 4.17 shows the variances of the concentric ellipses center estimate when 20 data points are sampled randomly from each of the concentric ellipses boundaries and $\xi^{2}$ varies from $10^{-4}$ to 0.1 . In $(4.21)$, the noise covariance $\mathbf{Q}$ is set proportional to an identity matrix for the proposed method. The estimation variance of the O'Leary method $(\bigcirc$ symbol $)$ is always higher than the one of the proposed method $(\times$ symbol $)$

by about $2 \mathrm{~dB}$ when $\xi^{2} \leq 10^{-1.6}$. This is consistent with the simulation results in Fig. 4.7 and Fig. 4.9. Since the noise in the data points is quantized, the parameter variances of both methods vary only slightly as the noise power increases and they jump to much higher values when the noise power exceeds $10^{-1.6}$.

Fig. 4.18 shows the estimation performance of the $\boldsymbol{\Gamma}_{1}$ and $\varepsilon$ parameters. The upper set of results is for $\varepsilon$ and the lower one is for $\boldsymbol{\Gamma}_{1}$. Since the Gaussian noise added here is white $(\delta=0)$, we do not observe the performance improvement from the proposed method as indicated in Fig. 4.8 and Fig. 4.10 where the Gaussian noise is anisotropic with a correlation coefficient $\delta=-0.6$. If correlated Gaussian noise is used, the performance improvement will be more apparent.

\section{Eye Images}

One main application of ellipse fitting is in biometrics for iris recognition $[58,59]$. Iris recognition requires accurate extraction of the inner and outer boundaries of the iris (pupil and limbus). We shall show below the experimental results of applying the proposed concentric ellipses estimator with $\mathbf{Q}=\mathbf{I}$ in (4.21) to the edge data points extracted from an iris image. The iris (eye) images are from the CASIA-IrisV3 iris 
database with a resolution of $320 \times 280$ pixels. The images are processed directly without adding any noise. However, due to the blurring behavior in the images, the exacted edges will have inherent noise. Each figure to be presented has four subfigures. The upper left subfigure shows all the edges detected from the original eye image using the Canny edge detection technique [57]. The upper right subfigure shows only the edges belonging to the inner and outer boundaries of the iris. The lower left subfigure displays the original eye image with the estimated concentric ellipses from the proposed method superimposed. The lower right subfigure shows the ellipses obtained by other methods. $N_{1}=N_{2}=25$ data points are sampled randomly from each of the iris inner and outer boundaries for the estimation.

Fig. 4.19 represents an eye image that contains the whole iris inner boundary and only a portion in the outer boundary. The advantage of the concentric ellipses fitting over the single ellipse fitting is clear in this case. The lower left plot of Fig. 4.19 shows the accurate fitted concentric ellipses from the proposed method. The lower right plot shows two fitted ellipses obtained separately from the method derived by Harker and O'Leary [60]. Both methods fit the inner iris boundary very well. However, the single ellipse fitting method has difficulty for the iris outer boundary and gives wrong result, while the proposed method fits the outer ellipse quite well.

Fig. 4.20 compares concentric ellipses fitting between the proposed method (lower left) and the O'Leary method [10] (lower right) for this particular eye image in which the edges are relatively clean as shown in the upper right plot. Both methods give comparable results in this case.

Fig. 4.21 gives the results when the data points from the iris outer boundary are a little noisier than those in Fig. 4.20. Comparing the fitted ellipses from the proposed 
method (lower left) and the O'Leary method (lower right) shows that the common ellipse center estimate from the O'Leary method shifts to the left and it performs a little worse than the proposed method, especially on the iris inner boundary.

Fig. 4.22 depicts the results when the outer ellipse data points are quite noisy. The fitted ellipses from the proposed method (lower left) are much better than those from the O'Leary method (lower right). The common ellipse center estimated from the O'Leary method is much worse than the one from the proposed method.

To provide better measures of the performance comparison, Table 4.4 shows the total-square errors between the 50 measurement points and their closest points from the fitted ellipses for Figs. 4.20 - 4.22. The second column is from the proposed method and the third column is from the O'Leary method. The relative percentage reduction in the total-square errors of the proposed method relative to the O'Leary method is shown in the fourth column. The amount of improvement increases as the extracted edge data points become noisier due to the inherent characteristics of the images. For the image in Fig. 4.22, a $32.79 \%$ error reduction is achieved.

\subsection{Concluding Remarks}

This paper provides computationally efficient solutions for the fitting of concentric circles as well as for concentric ellipses. The proposed estimators have explicit solutions and are derived through equation error formulation and non-linear parameter transformation. They can be in non-iterative forms by setting the weighting matrix to identity. The non-iterative form has better computational efficiency than the other

non-iterative solutions from the literature and achieves comparable accuracy. The 
iterative form is self-initialized and provides better performance. The KCR lower bounds for the parameters of concentric circles and concentric ellipses are provided. The first order analysis shows that the proposed estimators in iterative form approach statistical efficiency asymptotically. Compared to the other iterative solutions from the literature, the proposed estimators appear to have higher noise tolerance level for the thresholding effect. Simulations on synthetic data and experimental results from real images validate the theoretical developments and the promising performance of the proposed estimators.

\subsection{Appendix}

The monic solution we proposed requires $k^{o}$ to be not near zero. This requirement can be easily fulfilled by adding a large constant to the measurement data. Such an operation is linear and it only translates the concentric ellipses center $\mathbf{c}^{o}$ and changes the value of $k^{o}$. The other parameters $\Gamma_{1}^{o}$ and $\varepsilon^{o}$ would not be affected by this operation.

Let the translation be $\mathbf{f}=[f, f]^{T}$ and the transformed data is $\tilde{\mathbf{s}}_{i j}=\mathbf{s}_{i j}+\mathbf{f}$. The estimate of the unknown parameter vector in (4.22) for $\tilde{\mathbf{s}}_{i j}$ is

$$
\frac{\boldsymbol{\varphi}_{e}}{k}=\left[\frac{\boldsymbol{\Gamma}_{1}(\mathbf{c}+\mathbf{f})}{k}, \frac{A_{1}}{k}, \frac{\rho_{1}}{k}, \frac{B_{1}}{k}, \frac{\varepsilon-1}{\varepsilon k}\right]^{T} \triangleq \hat{\boldsymbol{\varphi}}_{e}
$$

Following the steps described after (4.22), the translated concentric ellipses center 
Table 4.1: Bias results (in dB) from the proposed concentric circles and concentric ellipses estimators with different weighting matrices. The results were obtained from the average of 10000 ensemble runs when the noise power was set to $10^{-4}$.

\begin{tabular}{cccc}
\hline Concentric circles & Bias & MSE & KCR lower bound \\
\hline $\mathbf{W}_{c}$ as in $(4.9)$ & $-38.98 \mathrm{~dB}$ & $-36.61 \mathrm{~dB}$ & $-36.69 \mathrm{~dB}$ \\
\hline $\mathbf{W}_{c}=\sigma^{2} \mathbf{I}$ & $-39.50 \mathrm{~dB}$ & $-34.15 \mathrm{~dB}$ & $-36.69 \mathrm{~dB}$ \\
\hline Concentric ellipses & Bias & MSE & KCR lower bound \\
\hline $\mathbf{W}_{c}$ as in $(4.21)$ & $-30.68 \mathrm{~dB}$ & $-30.76 \mathrm{~dB}$ & $-30.84 \mathrm{~dB}$ \\
\hline $\mathbf{W}_{c}=\sigma^{2} \mathbf{I}$ & $-25.93 \mathrm{~dB}$ & $-28.98 \mathrm{~dB}$ & $-30.84 \mathrm{~dB}$ \\
\hline
\end{tabular}

estimates from $\hat{\varphi}_{e}$ are

$$
\begin{aligned}
a+f & =\frac{\hat{\boldsymbol{\varphi}}_{e}(5) \hat{\boldsymbol{\varphi}}_{e}(1)-\hat{\boldsymbol{\varphi}}_{e}(4) \hat{\boldsymbol{\varphi}}_{e}(2)}{\hat{\boldsymbol{\varphi}}_{e}(3) \hat{\boldsymbol{\varphi}}_{e}(5)-\hat{\boldsymbol{\varphi}}_{e}(4)^{2}}, \\
b+f & =\frac{\hat{\boldsymbol{\varphi}}_{e}(3) \hat{\boldsymbol{\varphi}}_{e}(2)-\hat{\boldsymbol{\varphi}}_{e}(4) \hat{\boldsymbol{\varphi}}_{e}(1)}{\hat{\boldsymbol{\varphi}}_{e}(3) \hat{\boldsymbol{\varphi}}_{e}(5)-\hat{\boldsymbol{\varphi}}_{e}(4)^{2}} .
\end{aligned}
$$

From (4.52), we can see that the effect of $k$ in $\hat{\varphi}_{e}$ has been canceled out in the translated concentric ellipses center estimates. The estimate of $k^{o}$ is

$$
k=\frac{1}{(a+f) \hat{\boldsymbol{\varphi}}_{e}(1)+(b+f) \hat{\boldsymbol{\varphi}}_{e}(2)-1} .
$$

After finding $k$, it is straightforward to obtain the estimates of $\boldsymbol{\Gamma}_{1}^{o}$ and $\varepsilon^{o}$ and they are seen to be unaffected by the linear operation of the measurement data. The actual concentric ellipses center is estimated as its translated version subtracts the large constant term $\mathbf{f}$ that is known to us. 
Table 4.2: Computation time of the non-iterative and iterative solutions of the proposed estimators, the O'Leary method, the Kåsa method for concentric circles and concentric ellipses fittings. The results are relative to the proposed non-iterative solution and are obtained through 10000 ensemble runs at the noise power of $10^{-4}$.

\begin{tabular}{llccc}
\hline $\begin{array}{l}\text { Concentric } \\
\text { circles }\end{array}$ & $\begin{array}{l}\text { Proposed } \\
\text { non- } \\
\text { iterative } \\
\text { solution }\end{array}$ & O'Leary method [10] & Kåsa method [23] & $\begin{array}{l}\text { Proposed } \\
\text { iterative } \\
\text { solution }\end{array}$ \\
\hline \multicolumn{1}{c}{$\begin{array}{c}\text { Concentric } \\
\text { ellipses }\end{array}$} & $\begin{array}{l}\text { Proposed } \\
\text { non- } \\
\text { iterative } \\
\text { solution }\end{array}$ & O'Leary method [10] & Taubin method [31] & $\begin{array}{l}\text { Proposed } \\
\text { iterative } \\
\text { solution }\end{array}$ \\
\hline & 1 & & & 2.56 \\
\hline
\end{tabular}

Table 4.3: Averaged performance difference (in $\mathrm{dB}$ ) with the KCR lower bounds of different estimators for Figs. 4.3 to 4.6. The values provided are obtained by averaging over the range of noise power from $10^{-4}$ to 0.1 .

\begin{tabular}{cccc}
\hline Non-iterative methods & Proposed estimator & O'Leary method [10] & Kåsa method [23] \\
\hline Fig. 4.3 & $2.79 \mathrm{~dB}$ & $2.84 \mathrm{~dB}$ & $1.46 \mathrm{~dB}$ \\
\hline Fig. 4.4 & $2.27 \mathrm{~dB}$ & $2.35 \mathrm{~dB}$ & $3.84 \mathrm{~dB}$ \\
\hline Single circle fitting & Proposed estimator & Kåsa method [23] & Pratt method [25] \\
\hline Fig. 4.5 & $0.04 \mathrm{~dB}$ & $1.59 \mathrm{~dB}$ & $1.60 \mathrm{~dB}$ \\
\hline Fig. 4.6 & $0.04 \mathrm{~dB}$ & $1.02 \mathrm{~dB}$ & $1.07 \mathrm{~dB}$ \\
\hline
\end{tabular}

Table 4.4: Comparisons of the total-square errors between the 50 data points and their closest points on the fitted ellipses from the proposed method and the O'Leary method.

\begin{tabular}{cccc}
\hline Figure & Proposed method & O'Leary method [10] & Relative reduction (\%) \\
\hline 4.20 & 59.5781 & 62.1671 & 4.16 \\
\hline 4.21 & 72.7397 & 85.8301 & 15.25 \\
\hline 4.22 & 162.3400 & 241.5422 & 32.79 \\
\hline
\end{tabular}




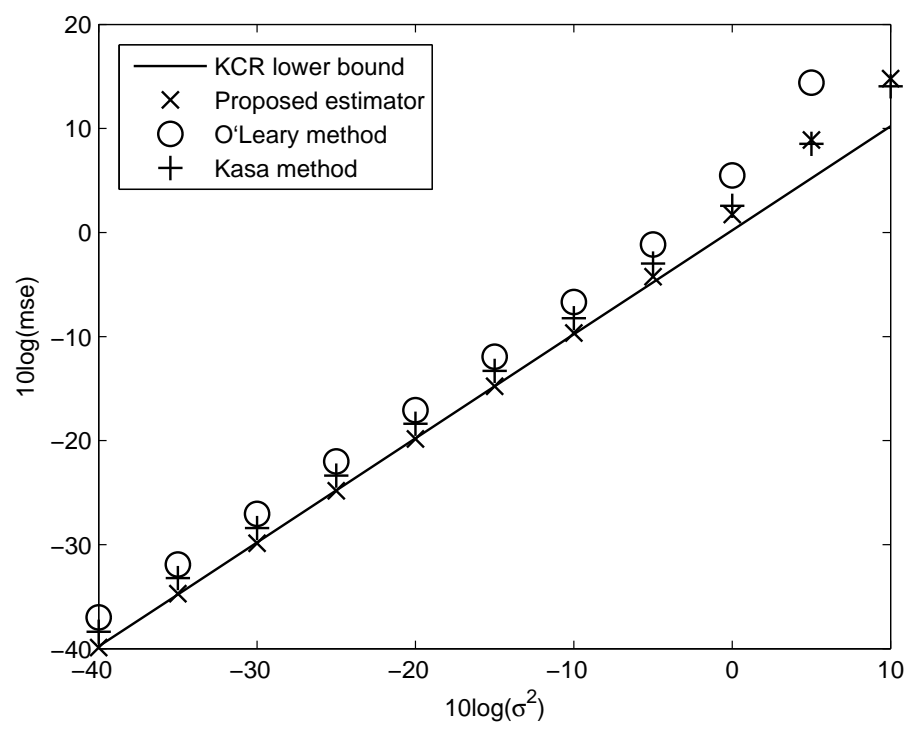

Figure 4.1: Comparison of the proposed estimator, the O'Leary method and the Kåsa method with the KCR lower bound for the estimations of the concentric circles center $\mathbf{c}^{o}=\left[\begin{array}{ll}0 & 0\end{array}\right]^{T}$ with data points from the two arc segments between 0 and $\pi$ rad when the noise power $\sigma^{2}$ varies, $\delta=0.8$ and $N_{1}=N_{2}=10$.

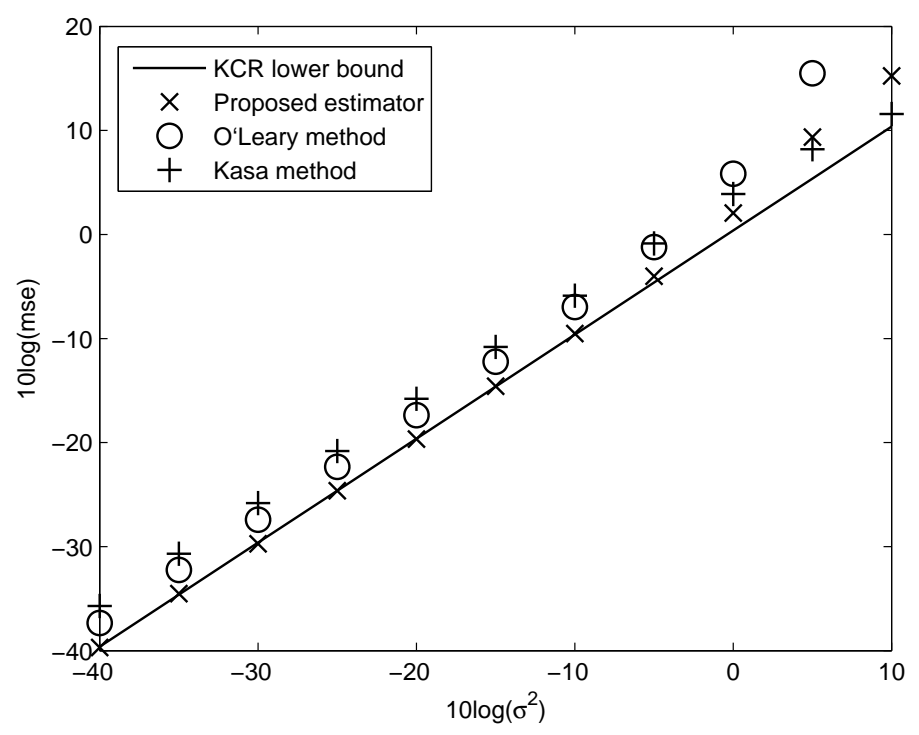

Figure 4.2: Comparison of the proposed estimator, the O'Leary method and the Kåsa method with the KCR lower bound for the estimations of the concentric circles radii $r_{1}^{o}=10$ and $r_{2}^{o}=20$ with data points from the two arc segments between 0 and $\pi$ rad when the noise power $\sigma^{2}$ varies, $\delta=0.8$ and $N_{1}=N_{2}=10$. 


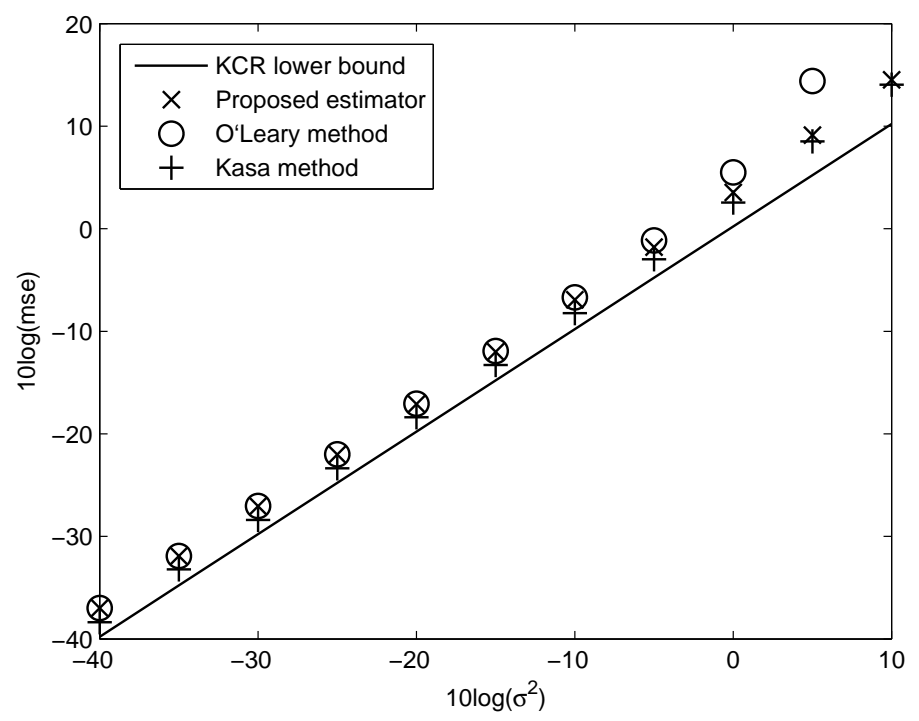

Figure 4.3: Comparison of the non-iterative solution from the proposed estimator, the O'Leary method and the Kåsa method with the KCR lower bound for the estimations of the concentric circles center $\mathbf{c}^{o}=\left[\begin{array}{ll}0 & 0\end{array}\right]^{T}$ with data points from the two arc segments between 0 and $\pi \mathrm{rad}$ when the noise power $\sigma^{2}$ varies, $\delta=0.8$ and $N_{1}=N_{2}=10$.

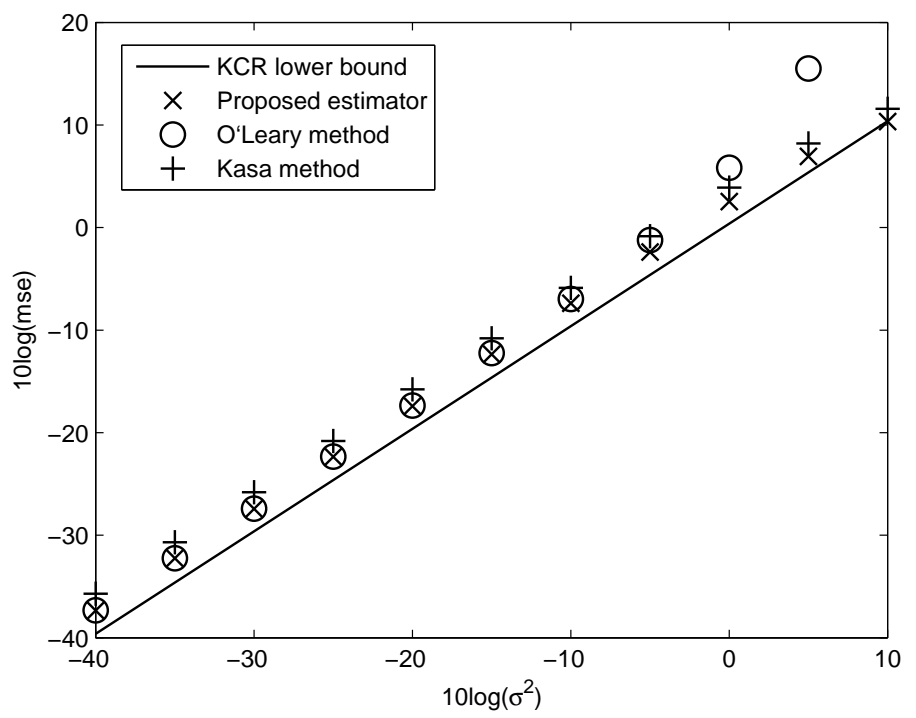

Figure 4.4: Comparison of the non-iterative solution from the proposed estimator, the O'Leary method and the Kåsa method with the KCR lower bound for the estimations of the concentric circles radii $r_{1}^{o}=10$ and $r_{2}^{o}=20$ with data points from the two arc segments between 0 and $\pi$ rad when the noise power $\sigma^{2}$ varies, $\delta=0.8$ and $N_{1}=N_{2}=10$. 


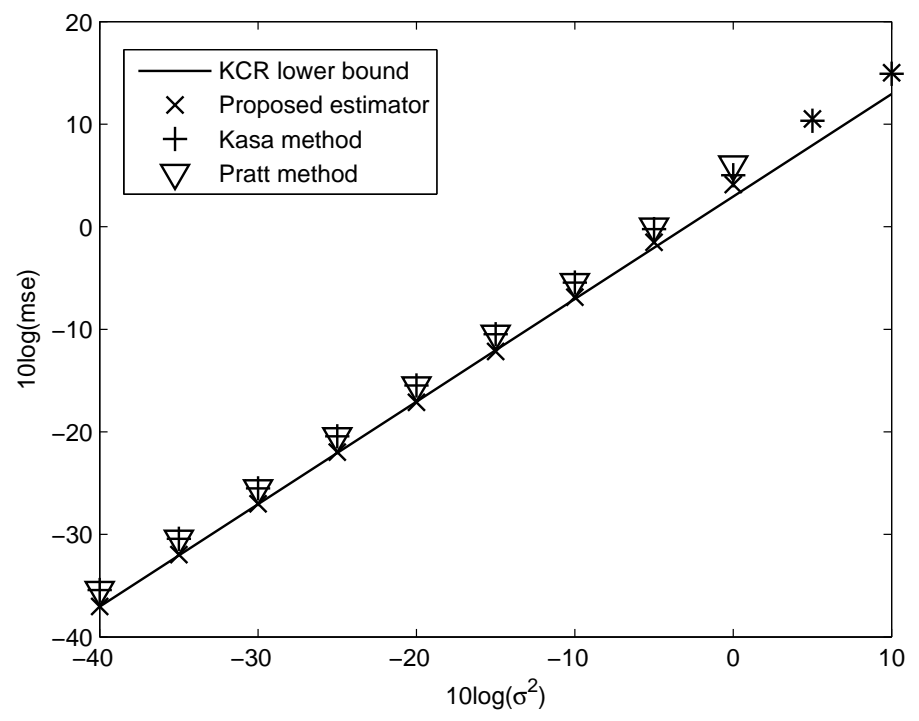

Figure 4.5: Comparison of the proposed estimator, the Kåsa method and the Pratt method with the KCR lower bound for the estimations of the single circle center $\mathbf{c}^{o}=\left[\begin{array}{ll}0 & 0\end{array}\right]^{T}$ when $\sigma^{2}$ varies, $\delta=0.8$ and $N_{1}=N_{2}=10$.

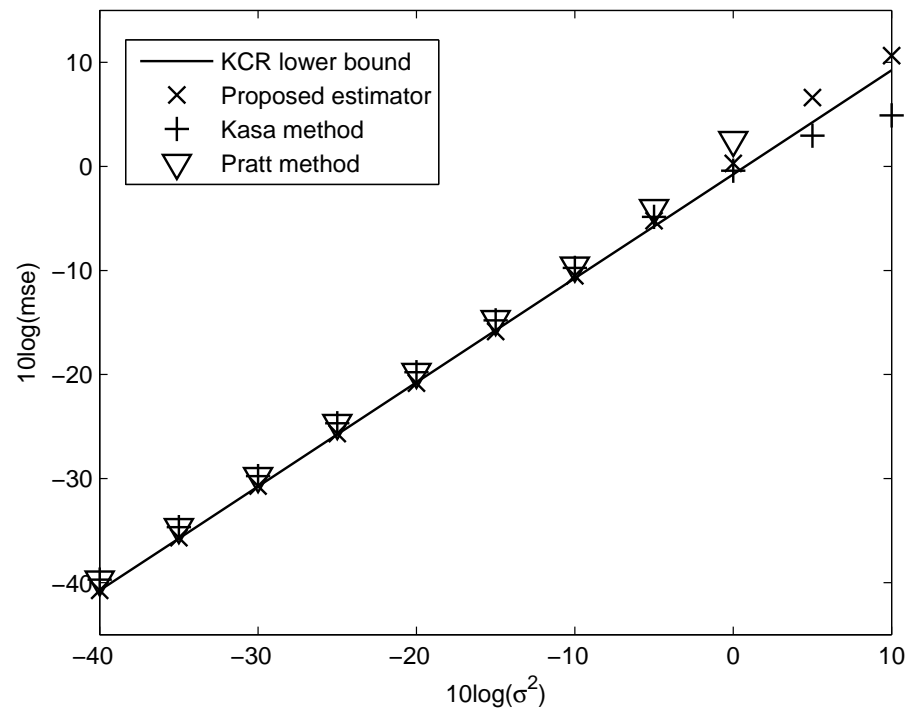

Figure 4.6: Comparison of the proposed estimator, the Kåsa method and the Pratt method with the KCR lower bound for the estimation of the single circle radius $r_{1}^{o}=10$ when $\sigma^{2}$ varies, $\delta=0.8$ and $N_{1}=N_{2}=10$. 


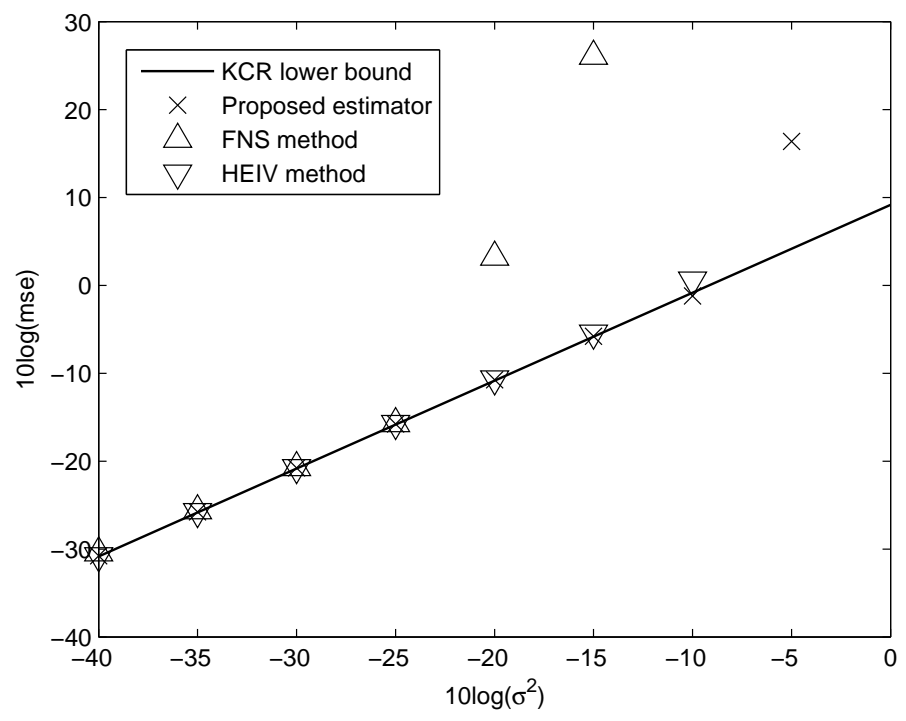

Figure 4.7: Comparison of the proposed estimator, the FNS method and the HEIV method with the KCR lower bound for the estimations of the concentric ellipses center $\mathbf{c}^{o}=\left[\begin{array}{ll}0 & 0\end{array}\right]^{T}$ when $\sigma^{2}$ varies from $10^{-4}$ to $1, N_{1}=N_{2}=10$ and $\delta=-0.6$. The data points are sampled from the concentric ellipses portions that are above the common major axis.

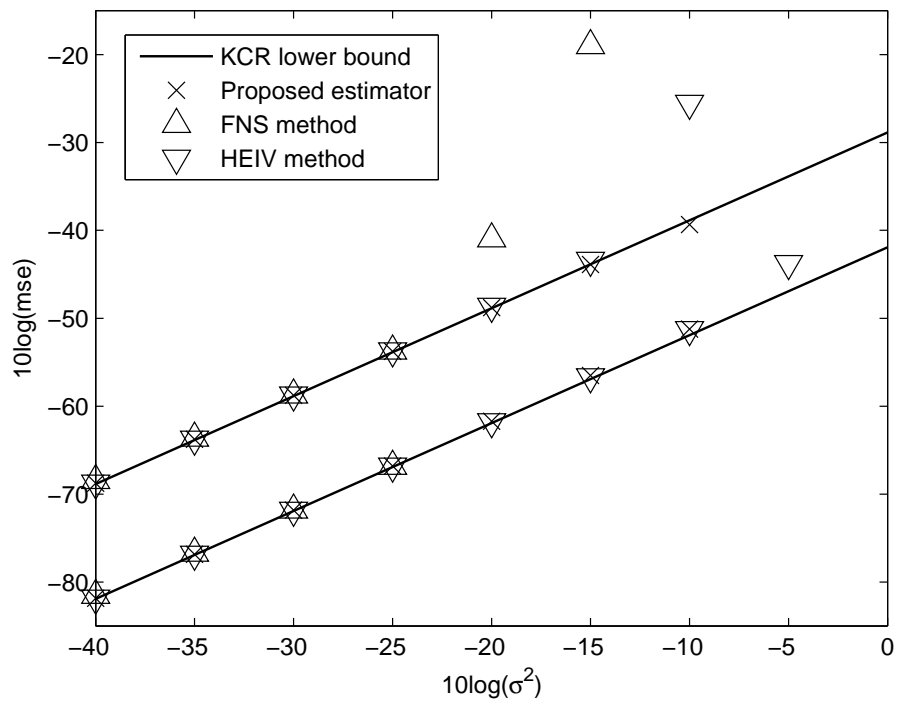

Figure 4.8: Comparison of the proposed estimator, the FNS method and the HEIV method with the KCR lower bound for the estimations of $\varepsilon^{o}=0.25$ (upper) and $\Gamma_{1}^{o}$ $\left(A_{1}^{o}=0.0075, B_{1}^{o}=0.0175\right.$ and $\rho_{1}^{o}=-0.0087$ ) (lower) when $\sigma^{2}$ varies from $10^{-4}$ to $1, N_{1}=N_{2}=10$ and $\delta=-0.6$. The data points are sampled from the concentric ellipses portions that are above the common major axis. 


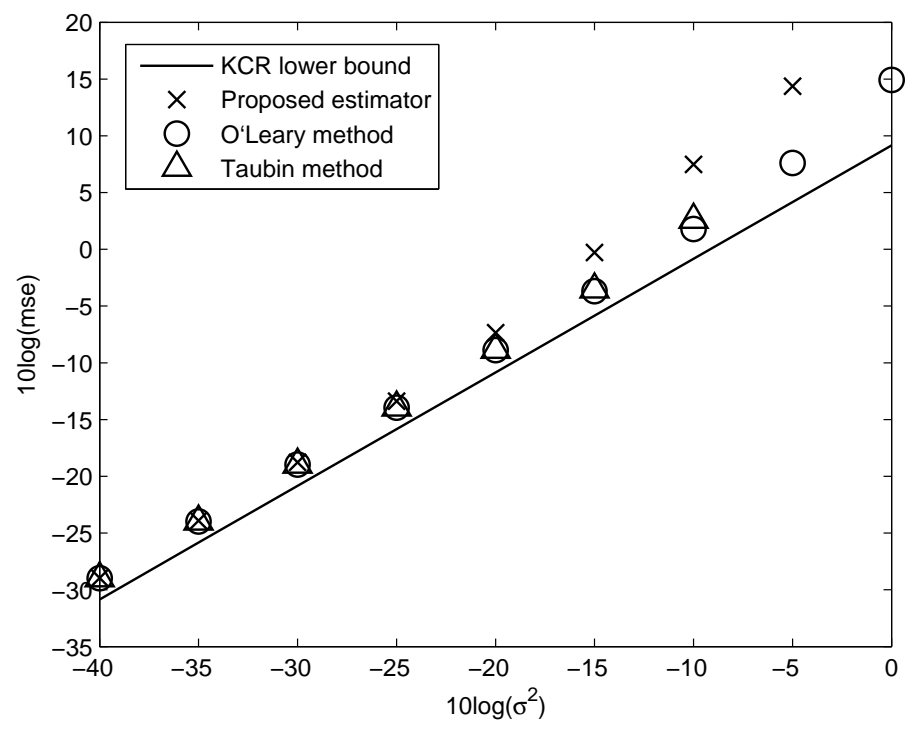

Figure 4.9: Comparison of the non-iterative solution of the proposed estimator, the O'Leary method and the Taubin method with the KCR lower bound for the estimations of the concentric ellipses center $\mathbf{c}^{o}=\left[\begin{array}{ll}0 & 0\end{array}\right]^{T}$ when $\sigma^{2}$ varies from $10^{-4}$ to 1 , $N_{1}=N_{2}=10$ and $\delta=-0.6$.

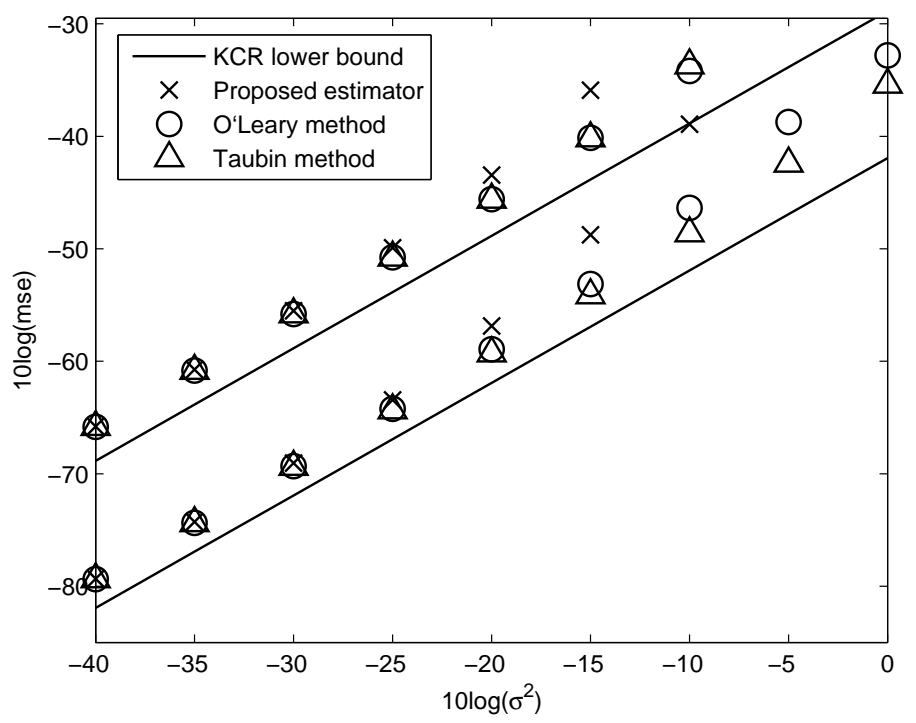

Figure 4.10: Comparison of the non-iterative solution of the proposed estimator, the O'Leary method and the Taubin method with the KCR lower bound for the estimations of $\varepsilon^{o}=0.25$ (upper) and $\Gamma_{1}^{o}\left(A_{1}^{o}=0.0075, B_{1}^{o}=0.0175\right.$ and $\left.\rho_{1}^{o}=-0.0087\right)$ (lower) when $\sigma^{2}$ varies from $10^{-4}$ to $1, N_{1}=N_{2}=10$ and $\delta=-0.6$. 


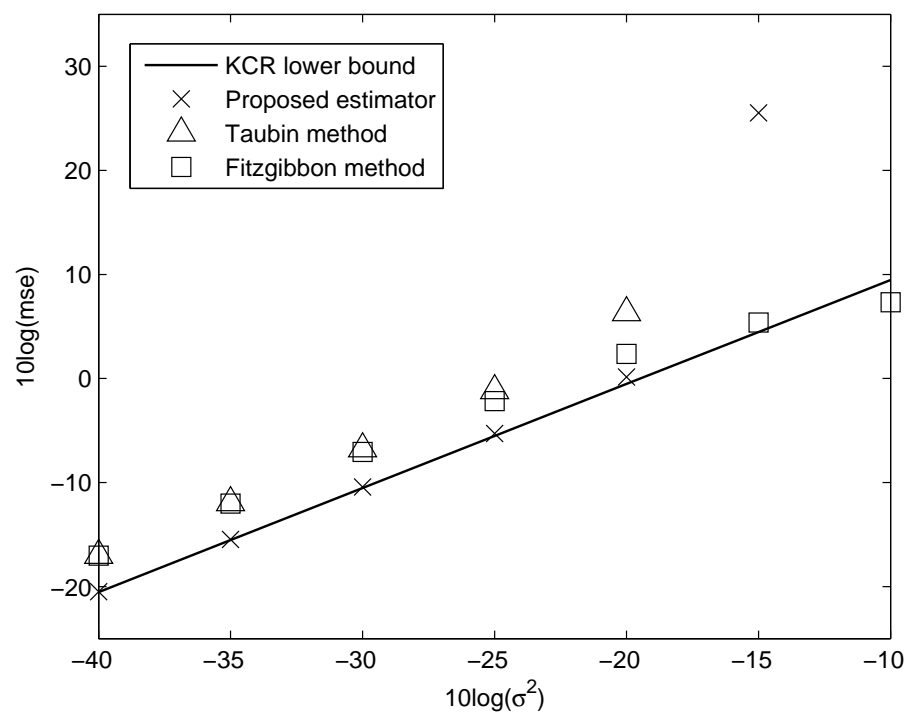

Figure 4.11: Comparison of the iterative solution of the proposed estimator, the Taubin method and the Fitzgibbon method with the KCR lower bound for the estimations of the single ellipse center $\mathbf{c}^{o}=\left[\begin{array}{ll}0 & 0\end{array}\right]^{T}$ when $\sigma^{2}$ varies from $10^{-4}$ to 0.1 , $N_{1}=10$ and $\delta=-0.6$. The data points are sampled from the single ellipse portion that is above the major axis.

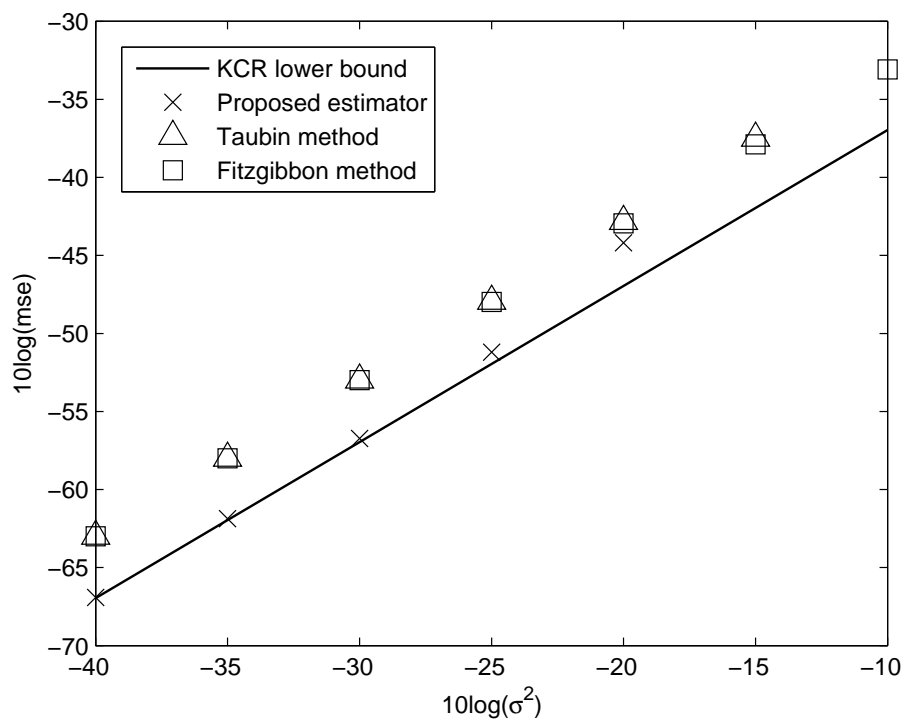

Figure 4.12: Comparison of the iterative solution of the proposed estimator, the Taubin method and the Fitzgibbon method with the KCR lower bound for the estimations of $\Gamma_{1}^{o}\left(A_{1}^{o}=0.0075, B_{1}^{o}=0.0175\right.$ and $\left.\rho_{1}^{o}=-0.0087\right)$ when $\sigma^{2}$ varies from $10^{-4}$ to $0.1, N_{1}=10$ and $\delta=-0.6$. The data points are sampled from the single ellipse portion that is above the major axis. 


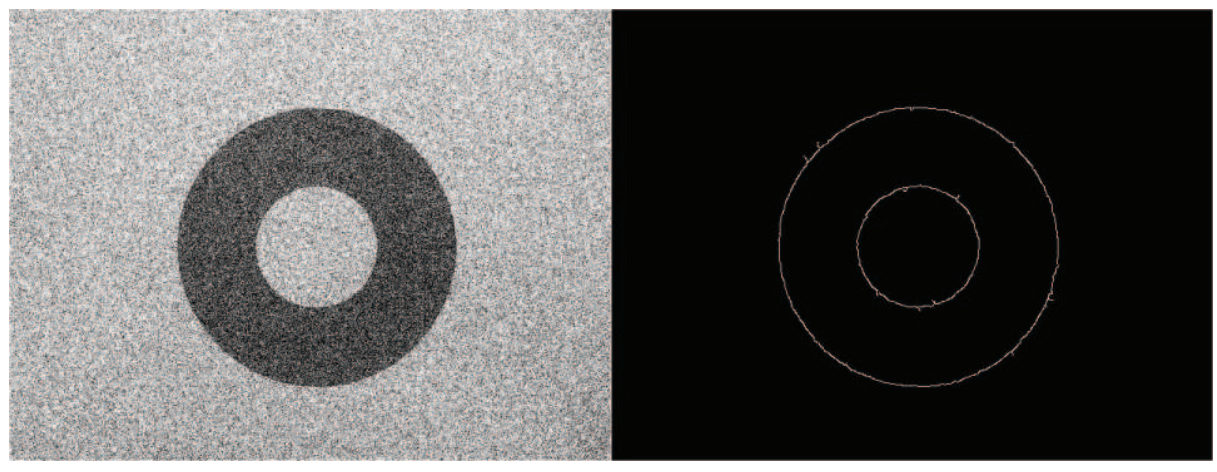

Figure 4.13: Original image of concentric circles with zero-mean Gaussian white noise added (left) and the corresponding image after edge detection (right).

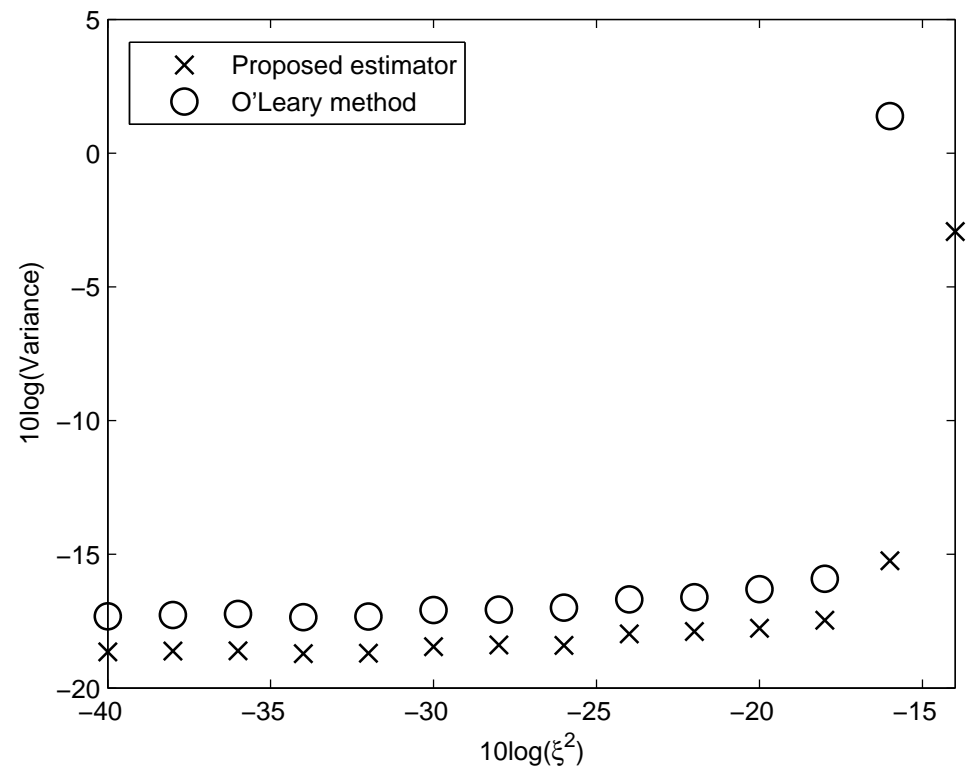

Figure 4.14: Comparison of the proposed estimator and the O'Leary method for the variance of the concentric circles center estimate when the noise power $\xi^{2}$ varies and $N_{1}=N_{2}=20$. 


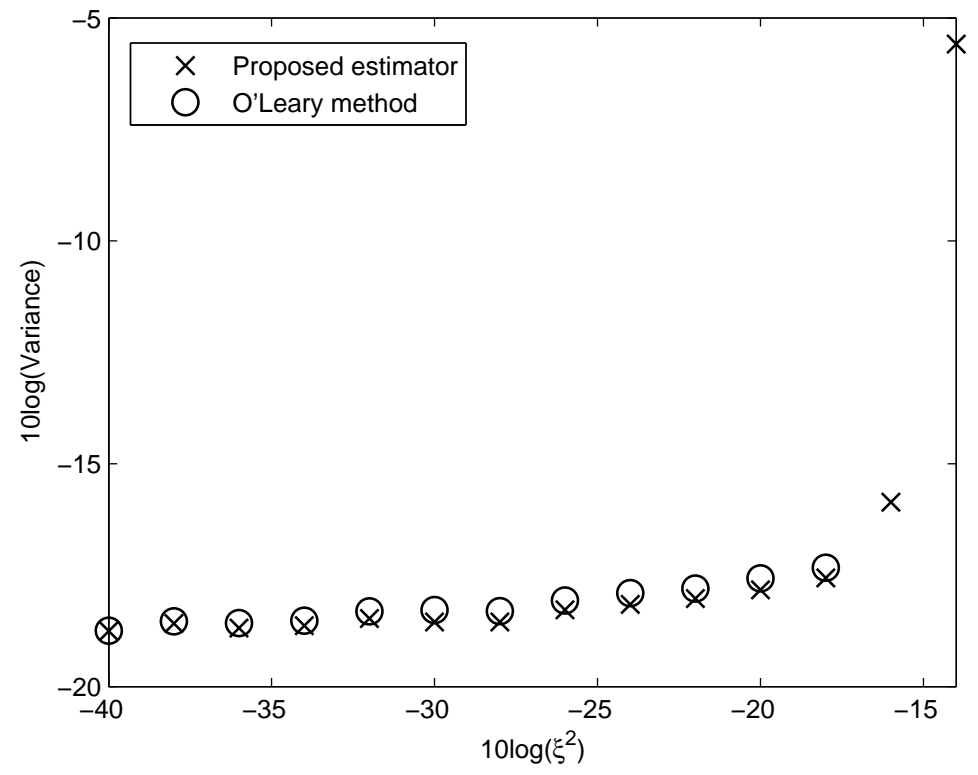

Figure 4.15: Comparison of the proposed estimator and the O'Leary method for the variance of the concentric circles radii estimate when the noise power $\xi^{2}$ varies and $N_{1}=N_{2}=20$.

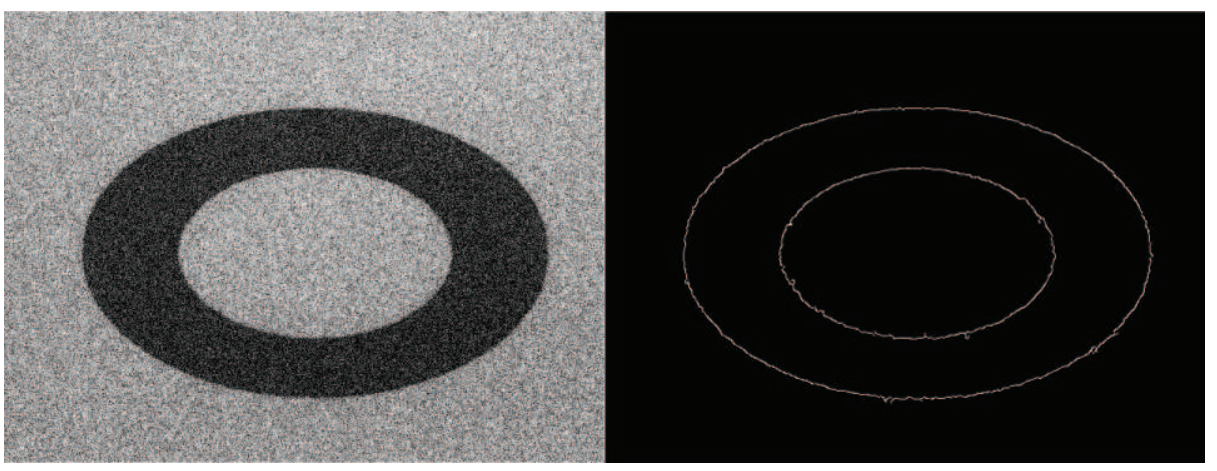

Figure 4.16: Original image of concentric ellipses with zero-mean Gaussian white noise added (left) and the corresponding image after edge detection (right). 


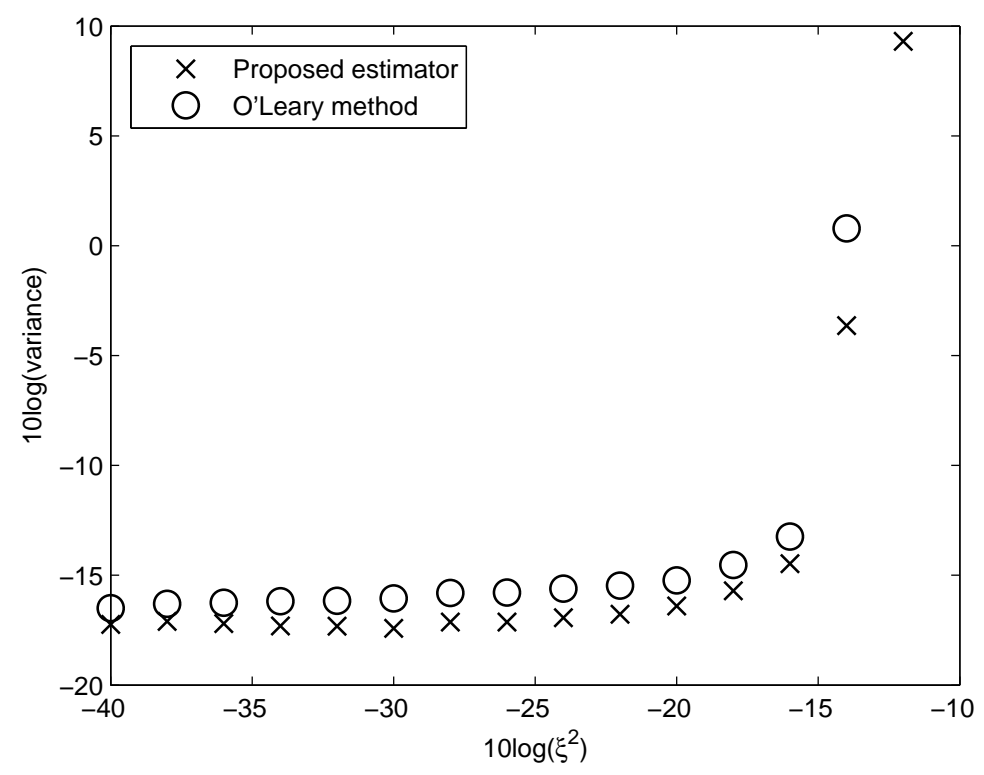

Figure 4.17: Comparison of the proposed estimator and the O'Leary method for the variance of the concentric ellipses center estimate when the noise power $\xi^{2}$ varies and $N_{1}=N_{2}=20$.

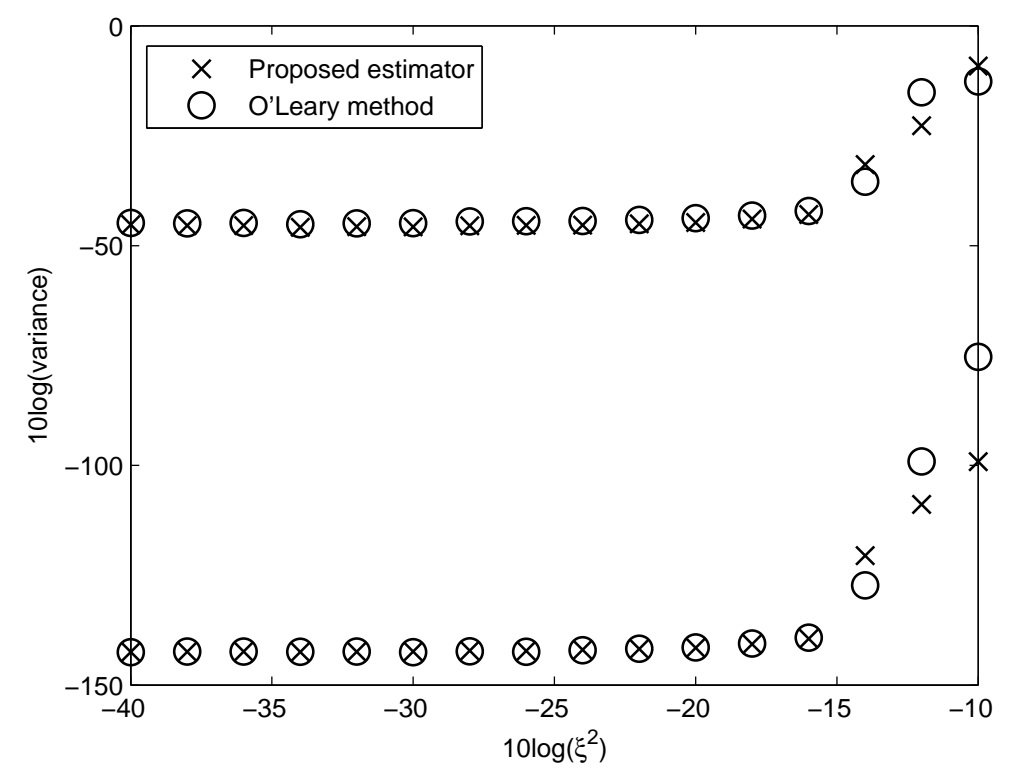

Figure 4.18: Comparison of the proposed estimator and the O'Leary method for the variance of the $\varepsilon$ (upper) and $\Gamma_{1}$ (lower) estimates when $\xi^{2}$ varies and $N_{1}=N_{2}=20$. 


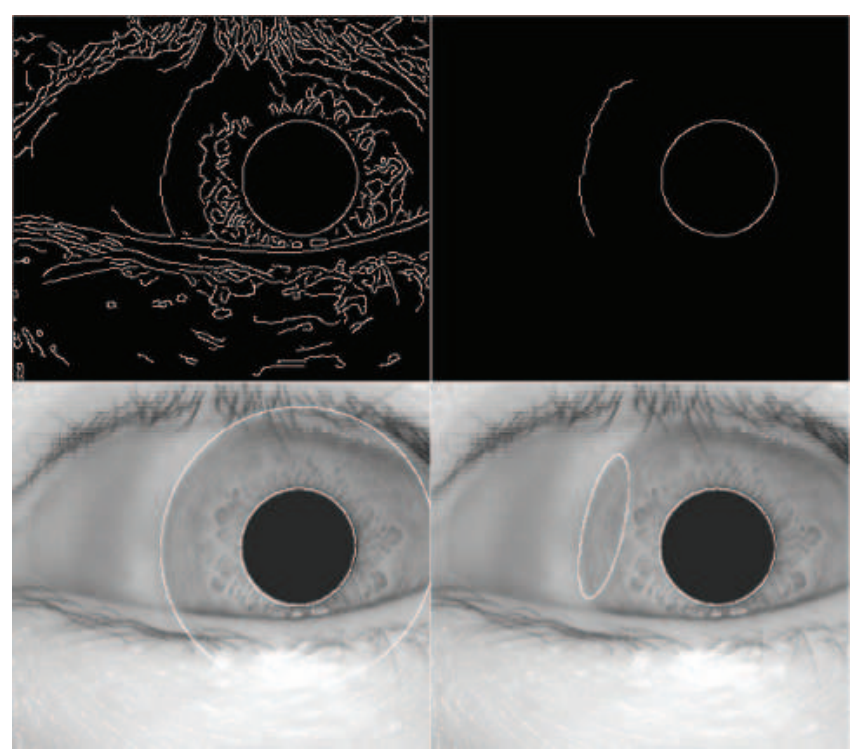

Figure 4.19: Results of an occlusive eye image: the output of Canny edge detection (upper left); the extracted iris boundaries (upper right); the estimated ellipses from the proposed estimator (lower left); the estimated ellipses by fitting inner and outer boundaries independently (lower right).
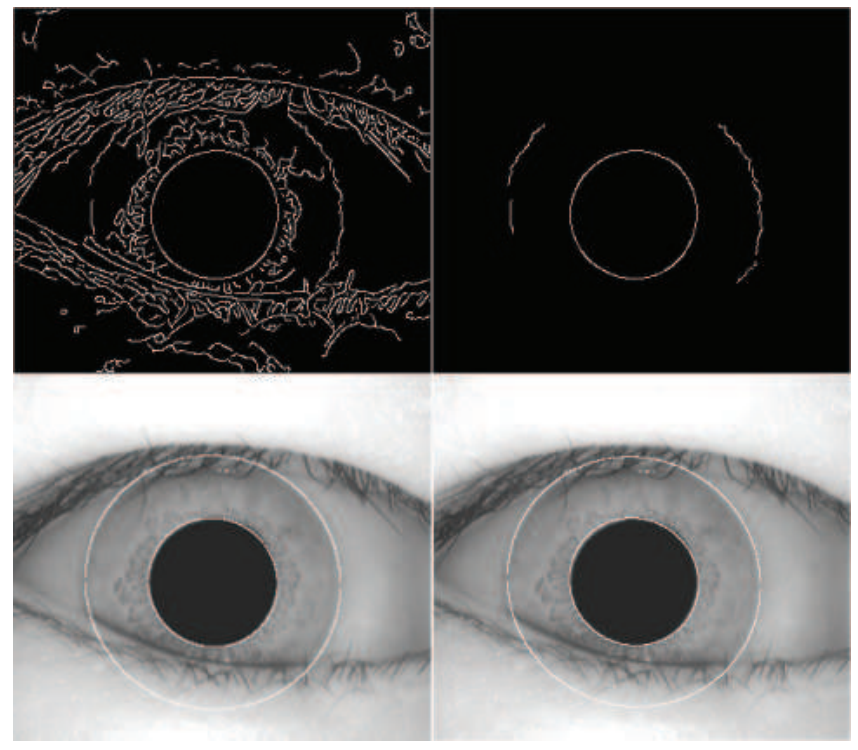

Figure 4.20: Results of an eye image with relatively clean detected edges: the output of Canny edge detection (upper left); the extracted iris boundaries (upper right); the estimated ellipses from the proposed estimator (lower left); the estimated ellipses from the O'Leary method (lower right). 


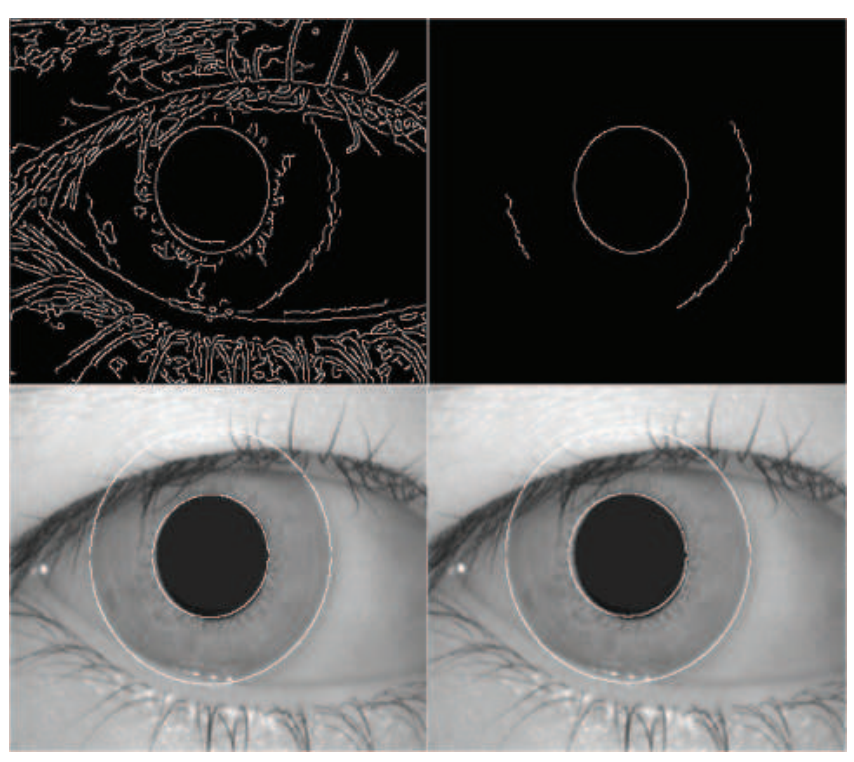

Figure 4.21: Results of an eye image with a little noisy detected edges: the output of Canny edge detection (upper left); the extracted iris boundaries (upper right); the estimated ellipses from the proposed estimator (lower left); the estimated ellipses from the O'Leary method (lower right).

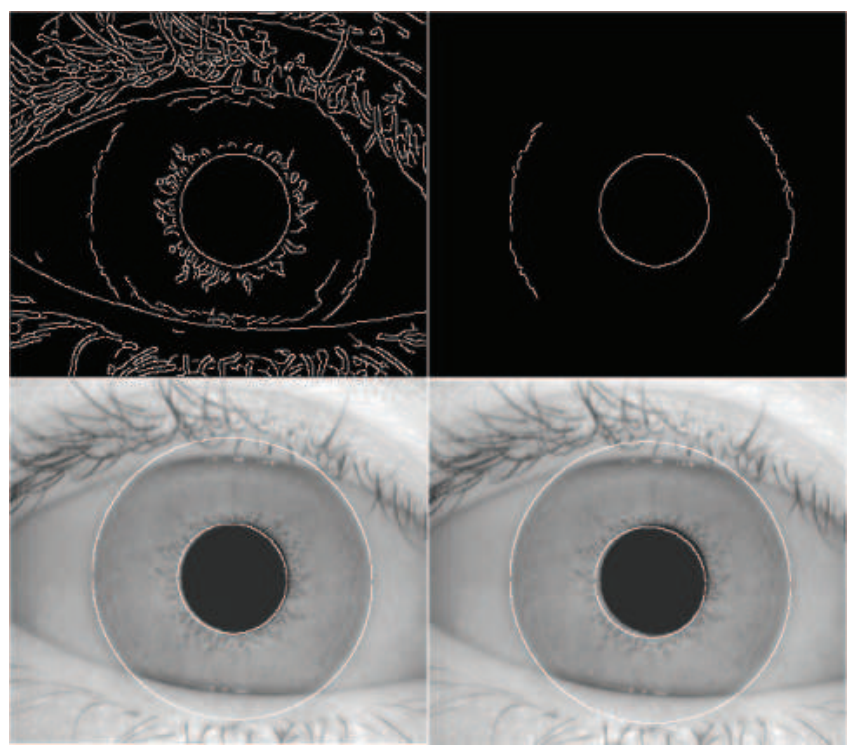

Figure 4.22: Results of an eye image with quite noisy detected edges: the output of Canny edge detection (upper left); the extracted iris boundaries (upper right); the estimated ellipses from the proposed estimator (lower left); the estimated ellipses from the O'Leary method (lower right). 


\section{Chapter 5}

\section{TOA Localization in the Presence}

\section{of Random Sensor Position Errors}

\subsection{TOA Localization Problem and Its CRLB and MSE Analysis}

The localization of a source via a collection of sensors is another classic and important nonlinear estimation problem. In this chapter the source localization problem based on TOA measurements in the presence of sensor position uncertainties is studied.

We consider one source and $M$ sensors in the localization scenario as shown in Fig. 1. The true source location $\mathbf{u}^{o}=\left[x^{o}, y^{o}, z^{o}\right]^{T}$ is unknown and it is to be estimated using the TOAs of the source signal received at the sensors. The true positions of the sensors $\mathbf{s}_{i}^{o}=\left[x_{i}^{o}, y_{i}^{o}, z_{i}^{o}\right]^{T}, i=1,2, \cdots, M$ are not known. The available sensor positions are $\mathbf{s}_{i}$ and they are inaccurate, i.e. $\mathbf{s}_{i}=\mathbf{s}_{i}^{o}+\mathbf{n}_{s i}$, where $\mathbf{n}_{s i}$ represents the position error of sensor $i$. They are collected to form a $3 M \times 1$ sensor position 
vector $\mathbf{s}=\left[\mathbf{s}_{1}^{T}, \mathbf{s}_{2}^{T}, \cdots, \mathbf{s}_{M}^{T}\right]^{T}=\mathbf{s}^{o}+\mathbf{n}_{s}$, where $\mathbf{s}^{o}$ is the true sensor position vector and $\mathbf{n}_{s}=\left[\mathbf{n}_{s 1}^{T}, \mathbf{n}_{s 2}^{T}, \cdots, \mathbf{n}_{s M}^{T}\right]^{T}$. We model $\mathbf{n}_{s}$ as a zero-mean Gaussian random vector with covariance matrix $\mathbf{Q}_{s}$.

The TOA observations are converted to the range measurements denoted by an $M \times 1$ range vector $\mathbf{r}=\left[r_{1}, r_{2}, \cdots, r_{M}\right]^{T}=\mathbf{r}^{o}+\mathbf{n}_{r}$, where $\mathbf{r}^{o}$ is the true range vector and $\mathbf{n}_{r}=\left[n_{r 1}, n_{r 2}, \cdots, n_{r M}\right]^{T}$ is the range measurement noise vector. $\mathbf{n}_{r}$ is also modeled as a zero-mean Gaussian random vector with covariance matrix $\mathbf{Q}_{r}$. We assume that $\mathbf{n}_{s}$ and $\mathbf{n}_{r}$ are independent of each other for the purpose of simplifying exemplification.

In order to better understand how the sensor position error affects the estimation accuracy of the source location, we evaluate the source location CRLB in the presence of sensor position errors. From data vector $\mathbf{d}=\left[\mathbf{r}^{T}, \mathbf{s}^{T}\right]^{T}$, the Fisher information matrix (FIM) of the unknown $\boldsymbol{\lambda}^{o}=\left[\mathbf{u}^{o T}, \mathbf{s}^{o T}\right]^{T}$ is $[6]$

$$
\mathrm{FIM}=-E\left[\frac{\partial^{2} \ln p\left(\mathbf{d} ; \boldsymbol{\lambda}^{o}\right)}{\partial \boldsymbol{\lambda}^{o} \partial \boldsymbol{\lambda}^{o T}}\right]
$$

where $\ln p\left(\mathbf{d} ; \boldsymbol{\lambda}^{o}\right)$ is the logarithm of the probability density function of $\mathbf{d}$ parameterized on $\boldsymbol{\lambda}^{o}$. (5.1) can be expressed using three block matrices $\mathbf{X}, \mathbf{Y}$ and $\mathbf{Z}$ as

$$
\mathrm{FIM}=\left[\begin{array}{cc}
\mathbf{X} & \mathbf{Y} \\
\mathbf{Y}^{T} & \mathbf{Z}
\end{array}\right]
$$

where

$$
\mathbf{X}=\left(\frac{\partial \mathbf{r}^{o}}{\partial \mathbf{u}^{o}}\right)^{T} \mathbf{Q}_{r}^{-1}\left(\frac{\partial \mathbf{r}^{o}}{\partial \mathbf{u}^{o}}\right), \mathbf{Y}=\left(\frac{\partial \mathbf{r}^{o}}{\partial \mathbf{u}^{o}}\right)^{T} \mathbf{Q}_{r}^{-1}\left(\frac{\partial \mathbf{r}^{o}}{\partial \mathbf{s}^{o}}\right), \mathbf{Z}=\left(\frac{\partial \mathbf{r}^{o}}{\partial \mathbf{s}^{o}}\right)^{T} \mathbf{Q}_{r}^{-1}\left(\frac{\partial \mathbf{r}^{o}}{\partial \mathbf{s}^{o}}\right)+\mathbf{Q}_{s}^{-1}
$$


Let $\mathbf{q}_{i}=\left(\mathbf{u}^{o}-\mathbf{s}_{i}^{o}\right) / r_{i}^{o}, i=1,2, \cdots, M$, then the $i$ th rows of $\left(\frac{\partial \mathbf{r}^{o}}{\partial \mathbf{u}^{o}}\right)$ and $\left(\frac{\partial \mathbf{r}^{o}}{\partial \mathbf{s}^{o}}\right)$ are equal to

$$
\left(\frac{\partial r_{i}^{o}}{\partial \mathbf{u}^{o}}\right)^{T}=\mathbf{q}_{i}^{T},\left(\frac{\partial r_{i}^{o}}{\partial \mathbf{s}^{o}}\right)^{T}=\left[\mathbf{0}_{3(i-1) \times 1}^{T},-\mathbf{q}_{i}^{T}, \mathbf{0}_{3(M-i) \times 1}^{T}\right]
$$

The CRLB of the source location corresponds to the upper left $3 \times 3$ submatrix of the inverse of the FIM. After applying the partitioned matrix inverse formula, we have

$$
\operatorname{CRLB}\left(\mathbf{u}^{o}\right)=\mathbf{X}^{-1}+\mathbf{X}^{-1} \mathbf{Y}\left(\mathbf{Z}-\mathbf{Y}^{T} \mathbf{X}^{-1} \mathbf{Y}\right)^{-1} \mathbf{Y}^{T} \mathbf{X}^{-1}
$$

The first term $\mathbf{X}^{-1}$ represents the source location CRLB when there is no sensor position error and the second term is the increase in the CRLB due to the presence of sensor position errors.

We next examine the MSE of the source location estimate from a Taylor-series based estimator [61] that pretends the known sensor positions are accurate but in fact they are erroneous. We shall let $f_{i}(\mathbf{u})=\left\|\mathbf{u}-\mathbf{s}_{i}\right\|, i=1,2, \cdots, M$ for simplicity. Suppose we have an initial guess of the source location $\check{\mathbf{u}}$ that is close to $\mathbf{u}^{o}$. Using the Taylor-series expansion up to linear term, we have

$$
\mathbf{f}(\mathbf{u}) \simeq \mathbf{f}(\check{\mathbf{u}})+\mathbf{F}(\check{\mathbf{u}})(\mathbf{u}-\check{\mathbf{u}})
$$

where $\mathbf{f}(\mathbf{u})=\left[f_{1}(\mathbf{u}), f_{2}(\mathbf{u}), \cdots, f_{M}(\mathbf{u})\right]^{T}$ and $\mathbf{F}(\check{\mathbf{u}})=\left.\frac{\partial \mathbf{f}(\mathbf{u})}{\partial \mathbf{u}}\right|_{\check{\mathbf{u}}}$.

Subtracting (5.6) from the range vector $\mathbf{r}$ produces the error $\mathbf{e}_{f}=\mathbf{r}-\mathbf{f}(\check{\mathbf{u}})-$ $\mathbf{F}(\check{\mathbf{u}})(\mathbf{u}-\check{\mathbf{u}})$ and $\mathbf{u}$ can be found by minimizing $\mathbf{e}_{f}^{T} \mathbf{Q}_{r}^{-1} \mathbf{e}_{f}$. Taking derivative with respect to $\mathbf{u}$, setting the gradient to zero and using $\mathbf{u}^{o}$ as $\check{\mathbf{u}}$ in the solution give

$$
\mathbf{u}-\mathbf{u}^{o}=\mathbf{U}^{-1} \mathbf{F}\left(\mathbf{u}^{o}\right)^{T} \mathbf{Q}_{r}^{-1}\left[\mathbf{r}-\mathbf{f}\left(\mathbf{u}^{o}\right)\right]
$$


where $\mathbf{U}=\left[\mathbf{F}\left(\mathbf{u}^{o}\right)^{T} \mathbf{Q}_{r}^{-1} \mathbf{F}\left(\mathbf{u}^{o}\right)\right]$. Note that $\mathbf{r}$ is with respect to the true sensor positions where as $\mathbf{f}\left(\mathbf{u}^{o}\right)$ is formed using the available sensor positions. Applying the Taylorseries expansion up to linear term on $\mathbf{f}\left(\mathbf{u}^{o}\right)$ at the true sensor positions and simplifying as in [13], we arrive at

$$
\mathbf{r}-\mathbf{f}\left(\mathbf{u}^{o}\right) \simeq \mathbf{n}_{r}-\mathbf{R n}_{s}
$$

where $\mathbf{R}$ is the same as $\left(\frac{\partial \mathbf{r}^{o}}{\partial \mathbf{s}^{o}}\right)$ which is defined in $(5.4)$.

Substituting (5.8) into (5.7) and multiplying by its transpose yield

$$
\operatorname{MSE}(\mathbf{u})=\mathbf{U}^{-1}+\mathbf{U}^{-1} \mathbf{F}\left(\mathbf{u}^{o}\right)^{T} \mathbf{Q}_{r}^{-1} \mathbf{R} \mathbf{Q}_{s} \mathbf{R}^{T} \mathbf{Q}_{r}^{-1} \mathbf{F}\left(\mathbf{u}^{o}\right) \mathbf{U}^{-1}
$$

In (5.6), the sensor position noise in $\mathbf{F}(\check{\mathbf{u}})$ is multiplied by the term $(\mathbf{u}-\check{\mathbf{u}})$, which will result in a second-order error term when $\check{\mathbf{u}}$ is close to the true sensor position $\mathbf{u}^{o}$. Therefore, the error resulting from replacing $\mathbf{s}_{i}$ by $\mathbf{s}_{i}^{o}$ in $\mathbf{F}(\check{\mathbf{u}})$ can be ignored. In such a case, $\mathbf{U}^{-1}$ in (5.9) is identical to $\mathbf{X}^{-1}$ in (5.5), the CRLB when the sensor positions are known exactly. The second term on the right hand side of (5.9) is the increase of inaccuracy caused by the sensor position errors. Using the source location and sensor position settings in Section 4, Fig. 5.3 shows the difference between the MSE and the CRLB for a distant and a near source. As the sensor position error power increases, the difference is about $3 \mathrm{~dB}$ and $5 \mathrm{~dB}$. This is a consequence of ignoring the sensor position errors in the estimation process. 


\subsection{Proposed Solution}

The performance loss is not negligible if the inaccuracy in sensor positions is ignored. We shall develop a new estimator that would take into account the sensor position errors and eventually improve the estimation of the source location. We follow the general procedure in [13] for the development.

First we look at the true range between the source and sensor $i, r_{i}^{o}=\left\|\mathbf{u}^{o}-\mathbf{s}_{i}^{o}\right\|, i=$ $1,2, \cdots, M$. Replacing $r_{i}^{o}$ by $r_{i}-n_{r i}, \mathbf{s}_{i}^{o}$ by $\mathbf{s}_{i}-\mathbf{n}_{s i}$ and taking square on both sides give

$$
r_{i}^{2}-2 r_{i}^{o} n_{r i}=\mathbf{s}_{i}^{T} \mathbf{s}_{i}+2\left(\mathbf{u}^{o}-\mathbf{s}_{i}\right)^{T} \mathbf{n}_{s i}-2 \mathbf{s}_{i}^{T} \mathbf{u}^{o}+\mathbf{u}^{o T} \mathbf{u}^{o}
$$

where the second order noise terms have been ignored. Let $e_{i}=2 r_{i}^{o} n_{r i}+2\left(\mathbf{u}^{o}-\mathbf{s}_{i}\right)^{T} \mathbf{n}_{s i}$ and $P_{i}=\mathbf{s}_{i}^{T} \mathbf{s}_{i}$. Rearranging the elements in (5.10) and collecting all $e_{i}$ yield

$$
\mathbf{e}=\mathbf{h}_{1}-\mathbf{G}_{1} \boldsymbol{\eta}_{1}^{o}
$$

where

$$
\mathbf{h}_{1}=\left[\begin{array}{c}
r_{1}^{2}-P_{1} \\
\vdots \\
r_{M}^{2}-P_{M}
\end{array}\right], \mathbf{G}_{1}=-2\left[\begin{array}{cc}
\mathbf{s}_{1}^{T} & -\frac{1}{2} \\
\vdots & \vdots \\
\mathbf{s}_{M}^{T}-\frac{1}{2}
\end{array}\right], \boldsymbol{\eta}_{1}^{o}=\left[\begin{array}{c}
\mathbf{u}^{o} \\
\mathbf{u}^{o T} \mathbf{u}^{o}
\end{array}\right]
$$

From the definition of $e_{i}$ below (5.10), we also have

$$
\mathbf{e}=\mathbf{V}_{1} \mathbf{n}_{r}+\mathbf{O}_{1} \mathbf{n}_{s}
$$


where

$$
\mathbf{V}_{1}=2\left[\begin{array}{ccc}
r_{1}^{o} & \cdots & 0 \\
\vdots & \ddots & \vdots \\
0 & \cdots & r_{M}^{o}
\end{array}\right], \mathbf{O}_{1}=2\left[\begin{array}{ccc}
\left(\mathbf{u}^{o}-\mathbf{s}_{1}\right)^{T} & \cdots & \mathbf{0}_{3 \times 1}^{T} \\
\vdots & \ddots & \vdots \\
\mathbf{0}_{3 \times 1}^{T} & \cdots & \left(\mathbf{u}^{o}-\mathbf{s}_{M}\right)^{T}
\end{array}\right]
$$

and $\mathbf{n}_{r}$ and $\mathbf{n}_{s}$ are the measurement noise vectors.

The equation (5.11) is nonlinear with respect to $\mathbf{u}^{o}$. But if we assume $\mathbf{u}^{o}$ and $\mathbf{u}^{o T} \mathbf{u}^{o}$ are independent with each other, it is linear in $\boldsymbol{\eta}_{1}^{o}$ and the weighted leastsquares (WLS) method [6] can be used to estimate $\boldsymbol{\eta}_{1}^{o}$ :

$$
\boldsymbol{\eta}_{1}=\left(\mathbf{G}_{1}^{T} \mathbf{W}_{1} \mathbf{G}_{1}\right)^{-1} \mathbf{G}_{1}^{T} \mathbf{W}_{1} \mathbf{h}_{1}
$$

where $\mathbf{W}_{1}=E\left[\mathbf{e e}^{T}\right]^{-1}$ and from (5.13)

$$
\mathbf{W}_{1}=\left(\mathbf{V}_{1} \mathbf{Q}_{r} \mathbf{V}_{1}^{T}+\mathbf{O}_{1} \mathbf{Q}_{s} \mathbf{O}_{1}^{T}\right)^{-1}
$$

If the sensor position error is small enough such that we can ignore the noise in $\mathbf{G}_{1}$, the covariance matrix of $\boldsymbol{\eta}_{1}$ is

$$
\operatorname{cov}\left(\boldsymbol{\eta}_{1}\right)=\left(\mathbf{G}_{1}^{T} \mathbf{W}_{1} \mathbf{G}_{1}\right)^{-1}
$$

The first three elements of $\boldsymbol{\eta}_{1}$ give an estimate of $\mathbf{u}^{o}$. However, this solution is not accurate because we assume $\mathbf{u}^{o}$ and $\mathbf{u}^{o T} \mathbf{u}^{o}$ are unrelated when solving for $\boldsymbol{\eta}_{1}$, but this is certainly not the case. Therefore, further processing is needed to refine the estimation result. 
Let $\Delta \boldsymbol{\eta}_{1}$ be the estimation error of $\boldsymbol{\eta}_{1}$. Then

$$
\boldsymbol{\eta}_{1}(1: 3) \odot \boldsymbol{\eta}_{1}(1: 3) \simeq \mathbf{u}^{o} \odot \mathbf{u}^{o}+2 \mathbf{u}^{o} \odot \Delta \boldsymbol{\eta}_{1}(1: 3) .
$$

Here symbol $\odot$ denotes the element by element multiplication and $\Delta \boldsymbol{\eta}_{1}(1: 3) \odot \Delta \boldsymbol{\eta}_{1}(1$ : 3) has been ignored. (5.18) together with $\boldsymbol{\eta}_{1}(4) \simeq \mathbf{u}^{o T} \mathbf{u}^{o}+\Delta \boldsymbol{\eta}_{1}(4)$ form

$$
\mathbf{V}_{2} \Delta \boldsymbol{\eta}_{1}=\mathbf{h}_{2}-\mathbf{G}_{2} \boldsymbol{\eta}_{2}^{o}
$$

where

$$
\begin{aligned}
\mathbf{V}_{2} & =\operatorname{diag}\left[2 x^{o}, 2 y^{o}, 2 z^{o}, 1\right], \\
\mathbf{h}_{2} & =\left[\boldsymbol{\eta}_{1}^{2}(1), \boldsymbol{\eta}_{1}^{2}(2), \boldsymbol{\eta}_{1}^{2}(3), \boldsymbol{\eta}_{1}(4)\right]^{T}, \\
\mathbf{G}_{2} & =\left[\begin{array}{lll}
1 & 0 & 0 \\
0 & 1 & 0 \\
0 & 0 & 1 \\
1 & 1 & 1
\end{array}\right], \boldsymbol{\eta}_{2}^{o}=\mathbf{u}^{o} \odot \mathbf{u}^{o}=\left[\begin{array}{c}
x^{o 2} \\
y^{o 2} \\
z^{o 2}
\end{array}\right] .
\end{aligned}
$$

At this point, (5.19) is linear with respect to $\boldsymbol{\eta}_{2}^{o}$. The WLS solution of $\boldsymbol{\eta}_{2}^{o}$ is equal to

$$
\boldsymbol{\eta}_{2}=\left(\mathbf{G}_{2}^{T} \mathbf{W}_{2} \mathbf{G}_{2}\right)^{-1} \mathbf{G}_{2}^{T} \mathbf{W}_{2} \mathbf{h}_{2}
$$

and the weighting matrix is

$$
\mathbf{W}_{2}=E\left[\left(\mathbf{V}_{2} \Delta \boldsymbol{\eta}_{1}\right)\left(\mathbf{V}_{2} \Delta \boldsymbol{\eta}_{1}\right)^{T}\right]^{-1}=\mathbf{V}_{2}^{-T} \operatorname{cov}\left(\boldsymbol{\eta}_{1}\right)^{-1} \mathbf{V}_{2}^{-1}
$$


where $\operatorname{cov}\left(\boldsymbol{\eta}_{1}\right)$ is given by (5.17). The final source location estimate is

$$
\mathbf{u}=\operatorname{diag}\left\{\operatorname{sign}\left[\boldsymbol{\eta}_{1}(1: 3)\right]\right\} \sqrt{\boldsymbol{\eta}_{2}} .
$$

Note that the true value $\mathbf{u}^{o}$ is needed in forming the weighting matrices $\mathbf{W}_{1}$ and $\mathbf{W}_{2}$. To handle this situation, we shall begin by setting $\mathbf{W}_{1}$ to identity to obtain an initial $\mathbf{u}$ estimate, from which an approximate $\mathbf{W}_{1}$ can be created to obtain $\boldsymbol{\eta}_{1}$ using (5.15). The $\mathbf{u}$ estimate in $\boldsymbol{\eta}_{1}$ can be used to replace the true source location in $\mathbf{W}_{2}$. Simulation results show that the performance degradation from this approximation process is insignificant.

\subsection{Simulation}

The simulation scenario contains $M=6$ sensors whose true positions are listed in Table 5.1. The source is either distant at $[2000,2500,3000]^{T}$ or near at $[600,650,550]^{T}$. Except for the evaluation of the CRLB, the true sensor positions are not used and only their erroneous observations are presented to an estimation algorithm.

The covariance matrix of the sensor position measurements is $\mathbf{Q}_{s}=\sigma_{s}^{2} \mathbf{J}$, where $\mathbf{J}=\operatorname{diag}[1,1,1,2,2,2,10,10,10,40,40,40,20,20,20,3,3,3] . \sigma_{s}^{2}$ is the sensor position error power and it varies between $10^{-6}$ and $1 . \mathbf{Q}_{r}=\sigma_{r}^{2} \mathbf{I}$ is the covariance matrix of the range measurements, where $\mathbf{I}$ is a $6 \times 6$ identity matrix, $\sigma_{r}^{2}$ is the range measurement noise power that is fixed to $10^{-4}$.

In Fig. 5.2, we compare the traces of $\operatorname{CRLB}(\mathbf{u})$ in the presence (solid line) and absence (dash line) of sensor position errors for the distant source. The comparison shows that the gap between them becomes larger and larger as $\sigma_{s}^{2}$ increases. The 
Table 5.1: True Sensor Positions

\begin{tabular}{|c|ccc|c|ccc|}
\hline Sensor $i$ & $x_{i}^{o}$ & $y_{i}^{o}$ & $z_{i}^{o}$ & Sensor $i$ & $x_{i}^{o}$ & $y_{i}^{o}$ & $z_{i}^{o}$ \\
\hline 1 & 300 & 100 & 150 & 4 & 350 & 200 & 100 \\
\hline 2 & 400 & 150 & 100 & 5 & -100 & 100 & -100 \\
\hline 3 & 300 & 500 & 200 & 6 & 200 & -300 & -200 \\
\hline
\end{tabular}

difference is about $10 \mathrm{~dB}$ when $\sigma_{s}^{2}=10^{-4}$ and it becomes about $28 \mathrm{~dB}$ when $\sigma_{s}^{2}$ goes to $10^{-2}$.

In the presence of sensor position errors, Fig. 5.3 examines the theoretical MSE(u) when ignoring sensor position errors when estimating the distant (cross symbol) and near (circle symbol) sources. The $\operatorname{CRLB}(\mathbf{u})$ from (5.5) is also given for comparison. The theoretical MSE deviates from the corresponding CRLB gradually as $\sigma_{s}^{2}$ increases. Eventually when $\sigma_{s}^{2}$ is larger than $10^{-3.2}$, the decrease in accuracy reaches a stable value of about $3 \mathrm{~dB}$ for the distant source and about $5 \mathrm{~dB}$ for the near source.

Fig. 5.4 displays the accuracy of the proposed solution for both the distant (up triangle symbol) and near (down triangle symbol) sources together with the CRLBs. The performance of the proposed method is indicated using $\operatorname{mse}(\mathbf{u})=\sum_{k=1}^{K} \| \mathbf{u}_{k}-$ $\mathbf{u}^{o} \|^{2} / K$, where $K=10^{4}$ is the total number of ensemble runs and $\mathbf{u}_{k}$ is the source location estimate at run $k$. The proposed method reaches the CRLB accuracy very well in estimating the source location in both cases.

\subsection{Concluding Remarks}

In this chapter, we first evaluate the CRLB of the source location estimated from TOA measurements in the presence of sensor position errors. The CRLB analysis indicates how sensitive is the source location estimate with respect to the inaccuracy in sensor positions. The source location MSE is derived for an estimator that pretends 
the error in sensor positions is absent. We develop a closed-form solution to improve the source location estimate when sensor position errors are present. At the end, simulation is presented to confirm that the proposed method is able to reach the CRLB accuracy.
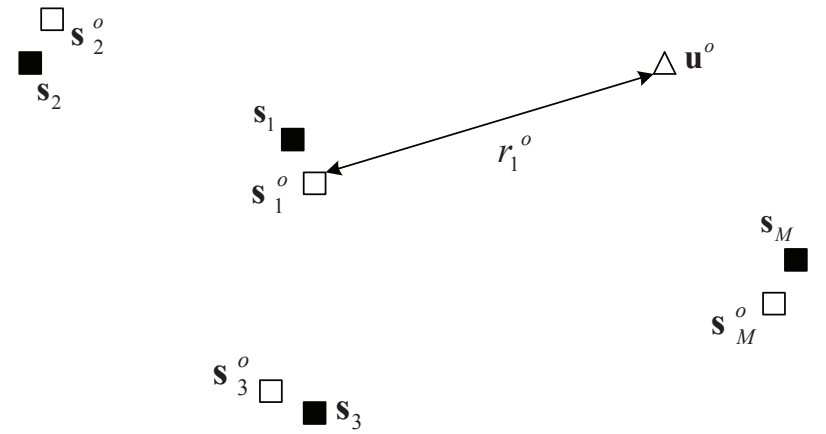

Figure 5.1: Localization of the source at $\mathbf{u}^{o}$ using a number of sensors at $\mathbf{s}_{i}$. Open squares denote the true sensor positions that are not known and closed squares are the available sensor positions that are erroneous.

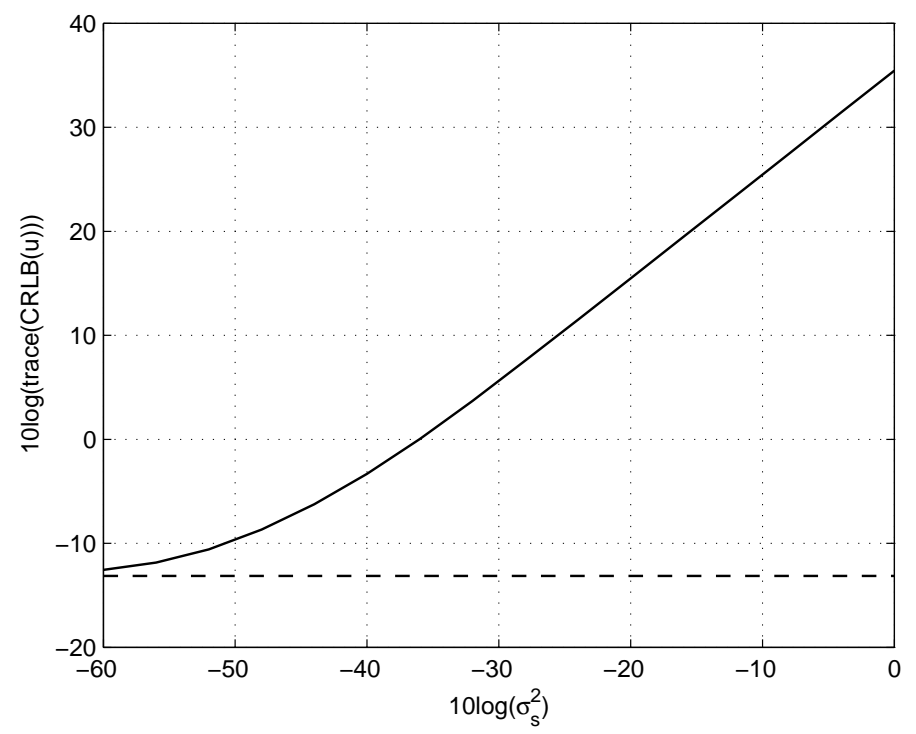

Figure 5.2: Comparison of the CRLB of a source location estimate in the presence (solid line) and the absence (dash line) of sensor position errors for the distant source. Only the traces of the CRLBs are shown. 


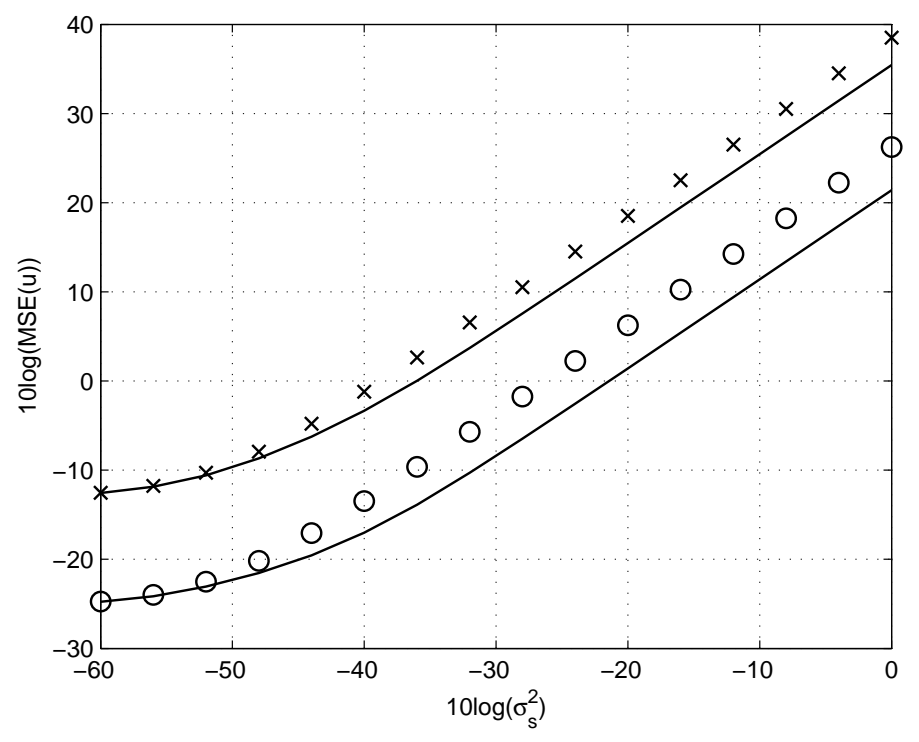

Figure 5.3: Theoretical MSE of the source location estimate from an algorithm that ignores sensor position errors, cross symbol for MSE of the distant source, circle symbol for MSE of the near source, solid lines for CRLB. Only the traces of MSE(u) and $\operatorname{CRLB}(\mathbf{u})$ are shown.

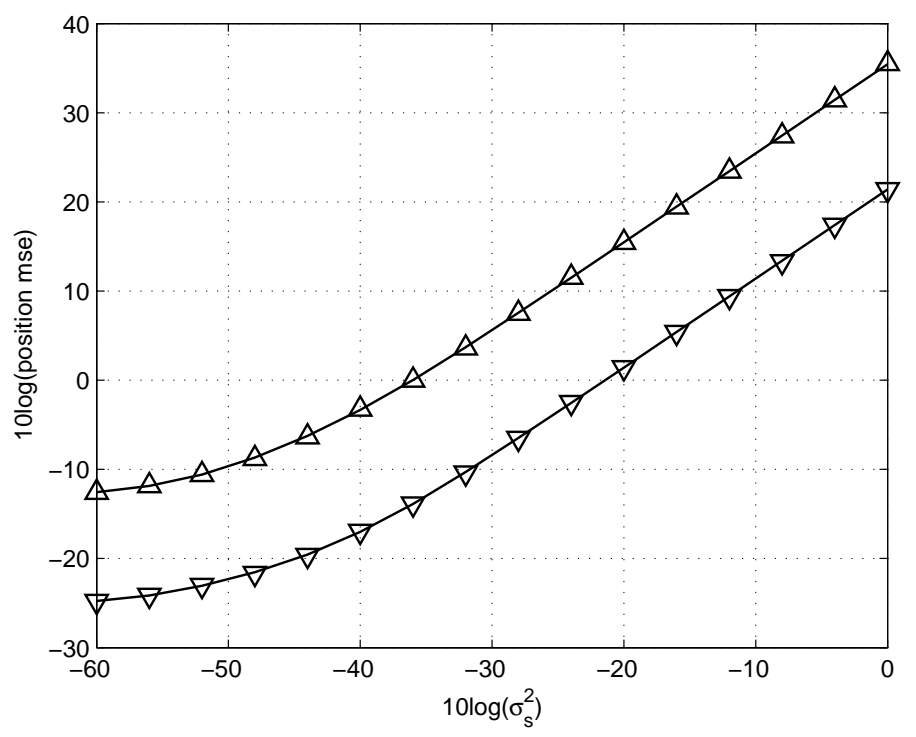

Figure 5.4: Performance of the proposed estimator for the distant (up triangle symbol) and the near (down triangle symbol) source with respect to the CRLB (solid line). Only the traces of the CRLBs are shown. 


\section{Chapter 6}

\section{Joint Source Localization and}

\section{Sensor Position Refinement for \\ Sensor Networks}

\subsection{Problem Formulation and Proposed Solution}

The study in Chapter 5 shows that the presence of sensor position errors would lead to a remarkable degradation in the source location estimate accuracy. A TOA based estimator is proposed in Chapter 5 to take into account the sensor position errors when estimating the source location. However, in addition to the source location, the erroneous sensor positions can also be estimated to further improve the source location estimate. In this chapter, by using the TOA measurements, a closed-form solution that jointly estimates the source and sensor positions is developed.

Let us consider the localization scenario as shown in Fig. 6.1, which consists of $M$ sensors to locate $K$ independent sources. The sources can be unknown emitters of 
interests or newly added sensor nodes. The true locations of the unknown sources to be found are denoted by $N \times 1$ vectors $\mathbf{u}_{i}^{o}, i=1,2, \cdots, K$, of Cartesian coordinates, where $N=2$ for $2 D$ localization or $N=3$ for $3 D$ localization. The precise positions of the sensors $\mathbf{s}_{j}^{o}, j=1,2, \cdots, M$, are not known and the available inaccurate sensor positions are $\mathbf{s}_{j}=\mathbf{s}_{j}^{o}+\Delta \mathbf{s}_{j}$, where $\Delta \mathbf{s}_{j}$ represents the position error of sensor $j$. They are collected to form a $N M \times 1$ sensor position vector $\mathbf{s}=\left[\mathbf{s}_{1}^{T}, \mathbf{s}_{2}^{T}, \cdots, \mathbf{s}_{M}^{T}\right]^{T}=\mathbf{s}^{o}+\Delta \mathbf{s}$, where $\mathbf{s}^{o}$ is the true sensor position vector and $\Delta \mathbf{s}=\left[\Delta \mathbf{s}_{1}^{T} \Delta \mathbf{s}_{2}^{T}, \cdots \Delta \mathbf{s}_{M}^{T}\right]^{T}$ is the random error vector. We shall model $\Delta \mathbf{s}$ as a zero-mean Gaussian random vector with covariance matrix $\mathbf{Q}_{s}$.

TOA measurements are commonly used in sensor networks. Assuming each sensor can acquire the signal from each source, we have, after multiplying with the signal propagation speed, the $M K \times 1$ measurement vector $\mathbf{r}=\left[\mathbf{r}_{1}^{T}, \mathbf{r}_{2}^{T}, \cdots, \mathbf{r}_{K}^{T}\right]^{T}=\mathbf{r}^{o}+\mathbf{n}$, where $\mathbf{r}^{o}$ is the true range vector and $\mathbf{n}$ is the noise vector. $\mathbf{r}_{i}=\left[r_{1, i}, r_{2, i} \cdots, r_{M, i}\right]^{T}$ is the measurement vector from source $i$ and $r_{j, i}$ is the TOA measurement of source $i$ to sensor $j . \mathbf{n}$ is modeled as a zero-mean Gaussian random vector with covariance matrix $\mathbf{Q}_{r}$. We shall assume $\Delta \mathbf{s}$ and $\mathbf{n}$ are independent of each other for ease of illustrations.

Our goal is to estimate the source locations and at the same time improve the inaccurate sensor positions as good as possible using the TOA measurements. The unknown parameter vector is $\boldsymbol{\theta}=\left[\mathbf{u}_{1}^{o T} \mathbf{u}_{2}^{o T}, \cdots, \mathbf{u}_{K}^{o T} \mathbf{s}^{o T}\right]^{T}$.

The proposed method makes use of a hypothetical source locations $\tilde{\mathbf{u}}_{i}=\mathbf{u}_{i}^{o}+\Delta \tilde{\mathbf{u}}_{i}$, where $\Delta \tilde{\mathbf{u}}_{i}$ is the difference between the hypothetical and the actual source location. The hypothetical locations are easy to obtain, please refer to [62] for details. 
We begin the algorithm development from the parametric form of $r_{j, i}^{o}$ :

$$
r_{j, i}^{o}=\left\|\mathbf{u}_{i}^{o}-\mathbf{s}_{j}^{o}\right\|
$$

Squaring both sides of (6.1), substituting $r_{j, i}^{o}=r_{j, i}-n_{j, i}, \mathbf{s}_{j}^{o}=\mathbf{s}_{j}-\Delta \mathbf{s}_{j}, \mathbf{u}_{i}^{o}=\tilde{\mathbf{u}}_{i}-\Delta \tilde{\mathbf{u}}_{i}$ and ignoring the second order terms of $n_{j, i}, \Delta \mathbf{s}_{j}$, and $\Delta \tilde{\mathbf{u}}_{i}$ yield

$$
\begin{aligned}
r_{j, i} n_{j, i}= & \frac{1}{2}\left[r_{j, i}^{2}-\mathbf{s}_{j}^{T}\left(\mathbf{s}_{j}-2 \tilde{\mathbf{u}}_{i}\right)\right]-\mathbf{s}_{j}^{T} \Delta \tilde{\mathbf{u}}_{i} \\
& -\frac{1}{2} \mathbf{u}_{i}^{o T} \mathbf{u}_{i}^{o}-\left(\tilde{\mathbf{u}}_{i}-\mathbf{s}_{j}\right)^{T} \Delta \mathbf{s}_{j} .
\end{aligned}
$$

We shall consider $\mathbf{u}_{i}^{o T} \mathbf{u}_{i}^{o}$ as a new independent unknown so that (6.2) becomes as a pseudo linear equation.

Other than the TOA measurements, the statistical knowledge of the sensor position errors $\Delta \mathbf{s}$ can also be utilized in the estimation. Following the technique from [63] and putting (6.2) together for $j=1,2 \cdots, M$ and $i=1,2, \cdots, K$ yield the matrix equation

$$
\boldsymbol{\epsilon}_{1}=\mathbf{h}_{1}-\mathbf{G}_{1} \varphi_{1}^{o}
$$


where

$$
\begin{aligned}
& \boldsymbol{\epsilon}_{1}=\left[\left(\mathbf{B}_{1} \mathbf{n}\right)^{T},-\Delta \mathbf{s}^{T}\right]^{T} \\
& \mathbf{B}_{1}=\operatorname{diag}\left\{\mathbf{B}_{1,1}, \mathbf{B}_{1,2}, \cdots, \mathbf{B}_{1, K}\right\}, \\
& \mathbf{B}_{1, i}=\operatorname{diag}\left\{r_{1, i}, r_{2, i}, \cdots, r_{M, i}\right\} \text {, } \\
& \mathbf{h}_{1}=\left[\boldsymbol{\eta}_{1}^{T}, \boldsymbol{\eta}_{2}^{T}, \cdots, \boldsymbol{\eta}_{K}^{T}, \mathbf{0}_{N M \times 1}^{T}\right]^{T}, \\
& \boldsymbol{\eta}_{i}=\frac{1}{2}\left[r_{1, i}^{2}-\mathbf{s}_{1}^{T}\left(\mathbf{s}_{1}-2 \tilde{\mathbf{u}}_{i}\right), \cdots, r_{M, i}^{2}-\mathbf{s}_{M}^{T}\left(\mathbf{s}_{M}-2 \tilde{\mathbf{u}}_{i}\right)\right]^{T}, \\
& \boldsymbol{\varphi}_{1}^{o}=\left[\Delta \tilde{\mathbf{u}}_{1}^{T}, \mathbf{u}_{1}^{o T} \mathbf{u}_{1}^{o}, \cdots, \Delta \tilde{\mathbf{u}}_{K}^{T}, \mathbf{u}_{K}^{o T} \mathbf{u}_{K}^{o}, \Delta \mathbf{s}^{T}\right]^{T}, \\
& \mathbf{G}_{1}=\left[\begin{array}{cccc}
\mathbf{G}_{1,1} & \cdots & \mathbf{O}_{M \times(N+1)} & \mathbf{D}_{1} \\
\vdots & \ddots & \vdots & \vdots \\
\mathbf{O}_{M \times(N+1)} & \cdots & \mathbf{G}_{1, K} & \mathbf{D}_{K} \\
\mathbf{O}_{N M \times(N+1)} & \cdots & \mathbf{O}_{N M \times(N+1)} & \mathbf{I}_{N M \times N M}
\end{array}\right] .
\end{aligned}
$$

$\mathbf{D}_{i}$ and $\mathbf{G}_{1, i}$ in $\mathbf{G}_{1}$ each has $M$ rows and their $j$ th rows, $j=1,2, \cdots, M$, are equal to $\left[\mathbf{0}_{N(j-1) \times 1}^{T},\left(\tilde{\mathbf{u}}_{i}-\mathbf{s}_{j}\right)^{T}, \mathbf{0}_{N(M-j) \times 1}^{T}\right]$ and $\left[\mathbf{s}_{j}^{T}, \frac{1}{2}\right]$.

The weighted least-squares (WLS) solution of $\varphi_{1}^{o}$ from the matrix equation (6.3) is

$$
\boldsymbol{\varphi}_{1}=\left(\mathbf{G}_{1}^{T} \mathbf{W}_{1} \mathbf{G}_{1}\right)^{-1} \mathbf{G}_{1}^{T} \mathbf{W}_{1} \mathbf{h}_{1}
$$

where the weighting matrix $\mathbf{W}_{1}$ is chosen to minimize the parameter estimation meansquare error:

$$
\mathbf{W}_{1}=\operatorname{diag}\left\{\mathbf{B}_{1} \mathbf{Q}_{r} \mathbf{B}_{1}, \mathbf{Q}_{s}\right\}^{-1}
$$

The estimation accuracy is characterized by the covariance of $\boldsymbol{\varphi}_{1}$, which is equal 


$$
\operatorname{cov}\left(\boldsymbol{\varphi}_{1}\right) \simeq\left(\mathbf{G}_{1}^{T} \mathbf{W}_{1} \mathbf{G}_{1}\right)^{-1}
$$

when the sensor position noise is relatively small and can be neglected in $\mathbf{G}_{1}$.

After $\varphi_{1}$ is obtained, the estimates of $\Delta \tilde{\mathbf{u}}_{i}$ and $\Delta \mathbf{s}$ can be represented as

$$
\begin{aligned}
\boldsymbol{\varphi}_{1, i} & \triangleq \boldsymbol{\varphi}_{1}((N+1)(i-1)+1:(N+1)(i-1)+N) \\
& =\Delta \tilde{\mathbf{u}}_{i}+\delta \tilde{\mathbf{u}}_{i}, \\
\boldsymbol{\varphi}_{1, s} & \triangleq \boldsymbol{\varphi}_{1}((N+1) K+1:(N+1) K+N M) \\
& =\Delta \mathbf{s}+\delta \mathbf{s},
\end{aligned}
$$

where $\delta \tilde{\mathbf{u}}_{i}$ and $\delta \mathbf{s}$ are the estimation errors of $\Delta \tilde{\mathbf{u}}_{i}$ and $\Delta \mathbf{s}$. Subtracting $\boldsymbol{\varphi}_{1, i}$ and $\boldsymbol{\varphi}_{1, s}$ from the hypothetical source location $\tilde{\mathbf{u}}_{i}$ and the sensor position vector $\mathbf{s}$ will provide the source and sensor position estimates. They are, however, not able to reach the CRLB accuracy. This is because we have introduced $K$ additional variables $\mathbf{u}_{i}^{o T} \mathbf{u}_{i}^{o}$, $i=1,2, \cdots, K$, in $\boldsymbol{\varphi}_{1}$. We next explore these $K$ additional variables to improve the estimation accuracy.

Though $\mathbf{u}_{i}^{o T} \mathbf{u}_{i}^{o}$ is not related to $\mathbf{s}^{o}$, the estimation errors of $\Delta \tilde{\mathbf{u}}_{i}$ and $\Delta \mathbf{s}$ in $\boldsymbol{\varphi}_{1}$ are correlated. As a result, when the accuracy of source location estimates is improved through the additional variables, the sensor position estimates can also be enhanced. In our stage- 2 solution, we will estimate the error terms $\delta \tilde{\mathbf{u}}_{i}$ and $\delta \mathbf{s}$ in (6.8) in order to provide more accurate estimations of the source locations and sensor positions.

The $(N+1) i$ th, $i=1,2 \cdots, K$, element of $\boldsymbol{\varphi}_{1}$ is the estimate of $\mathbf{u}_{i}^{o T} \mathbf{u}_{i}^{o}$

$$
\boldsymbol{\varphi}_{1}[(N+1) i]=\mathbf{u}_{i}^{o T} \mathbf{u}_{i}^{o}+\Delta \boldsymbol{\varphi}_{1}[(N+1) i]
$$


Putting $\mathbf{u}_{i}^{o}=\tilde{\mathbf{u}}_{i}-\boldsymbol{\varphi}_{1, i}+\delta \tilde{\mathbf{u}}_{i}$ into (6.9) gives

$$
\begin{aligned}
\Delta \boldsymbol{\varphi}_{1}[(N+1) i]= & \boldsymbol{\varphi}_{1}[(N+1) i]-\left(\tilde{\mathbf{u}}_{i}-\boldsymbol{\varphi}_{1, i}\right)^{T}\left(\tilde{\mathbf{u}}_{i}-\boldsymbol{\varphi}_{1, i}\right) \\
& -2\left(\tilde{\mathbf{u}}_{i}-\boldsymbol{\varphi}_{1, i}\right)^{T} \delta \tilde{\mathbf{u}}_{i} .
\end{aligned}
$$

Since $\delta \tilde{\mathbf{u}}_{i}=\Delta \boldsymbol{\varphi}_{1}[(N+1)(i-1)+1:(N+1)(i-1)+N]$ and $\delta \mathbf{s}=\Delta \boldsymbol{\varphi}_{1}[(N+1) K+1$ : $(N+1) K+N M]$, together with (6.10) we have the linear matrix equation

$$
\boldsymbol{\epsilon}_{2}=\mathbf{h}_{2}-\mathbf{G}_{2} \varphi_{2}^{o}
$$

where

$$
\begin{aligned}
& \boldsymbol{\epsilon}_{2}=\mathbf{B}_{2} \Delta \boldsymbol{\varphi}_{1}, \\
& \mathbf{B}_{2}=\operatorname{diag}\left\{\mathbf{B}_{2,1}, \mathbf{B}_{2,2}, \cdots, \mathbf{B}_{2, K},-\mathbf{I}_{N M \times N M}\right\}, \\
& \mathbf{B}_{2, i}=\mathbf{I}_{(N+1) \times(N+1)}, \mathbf{h}_{2}=\left[\boldsymbol{\xi}_{1}^{T}, \boldsymbol{\xi}_{2}^{T}, \cdots, \boldsymbol{\xi}_{K}^{T}, \mathbf{0}_{N M \times 1}^{T}\right]^{T}, \\
& \boldsymbol{\xi}_{i}=\left[\mathbf{0}_{N \times 1}^{T}, \boldsymbol{\varphi}_{1}((N+1) i)-\left(\tilde{\mathbf{u}}_{i}-\boldsymbol{\varphi}_{1, i}\right)^{T}\left(\tilde{\mathbf{u}}_{i}-\boldsymbol{\varphi}_{1, i}\right)\right]^{T}, \\
& \mathbf{G}_{2}=\operatorname{diag}\left\{\mathbf{G}_{2,1}, \mathbf{G}_{2,2}, \cdots, \mathbf{G}_{2, K}, \mathbf{I}_{N M \times N M}\right\}, \\
& \mathbf{G}_{2, i}=\left[-\mathbf{I}_{N \times N}, 2\left(\tilde{\mathbf{u}}_{i}-\boldsymbol{\varphi}_{1, i}\right)\right]^{T}, \\
& \boldsymbol{\varphi}_{2}^{o}=\left[\delta \tilde{\mathbf{u}}_{1}^{T}, \delta \tilde{\mathbf{u}}_{2}^{T}, \cdots, \delta \tilde{\mathbf{u}}_{K}^{T}, \delta \mathbf{s}^{T}\right]^{T} .
\end{aligned}
$$

The WLS solution of $\varphi_{2}^{o}$ is then

$$
\boldsymbol{\varphi}_{2}=\left(\mathbf{G}_{2}^{T} \mathbf{W}_{2} \mathbf{G}_{2}\right)^{-1} \mathbf{G}_{2}^{T} \mathbf{W}_{2} \mathbf{h}_{2}
$$


where the weighting matrix $\mathbf{W}_{2}$ is

$$
\mathbf{W}_{2}=\mathbf{B}_{2}^{-1} \operatorname{cov}\left(\boldsymbol{\varphi}_{1}\right)^{-1} \mathbf{B}_{2}^{-1}
$$

Let $\boldsymbol{\varphi}_{2, i}$ be $\boldsymbol{\varphi}_{2}[N(i-1)+1: N i]$ and $\boldsymbol{\varphi}_{2, s}$ be $\boldsymbol{\varphi}_{2}[N K+1: N K+N M]$. According to (6.8) the final source and sensor position estimates are

$$
\hat{\mathbf{u}}_{i}=\tilde{\mathbf{u}}_{i}-\left(\varphi_{1, i}-\varphi_{2, i}\right), \quad \hat{\mathbf{s}}=\mathbf{s}-\left(\varphi_{1, s}-\varphi_{2, s}\right)
$$

\subsection{Performance Analysis}

In this section we shall show analytically that the proposed solution can reach the CRLB accuracy. By using $\Delta \tilde{\mathbf{u}}_{i}=\tilde{\mathbf{u}}_{i}-\mathbf{u}_{i}^{o}$ and the definitions of $\boldsymbol{\varphi}_{1, i}, \boldsymbol{\varphi}_{1, s}$ in (6.8), (6.15) can be expressed as

$$
\begin{gathered}
\hat{\mathbf{u}}_{i}=\mathbf{u}_{i}^{o}-\left(\delta \tilde{\mathbf{u}}_{i}-\boldsymbol{\varphi}_{2, i}\right)=\mathbf{u}_{i}^{o}+\Delta \boldsymbol{\varphi}_{2, i}, \\
\hat{\mathbf{s}}=\mathbf{s}^{o}-\left(\delta \mathbf{s}-\boldsymbol{\varphi}_{2, s}\right)=\mathbf{s}^{o}+\Delta \boldsymbol{\varphi}_{2, s} .
\end{gathered}
$$

As a result, the covariance matrix of $\hat{\boldsymbol{\theta}}=\left[\hat{\mathbf{u}}_{1}^{T}, \cdots, \hat{\mathbf{u}}_{K}^{T}, \hat{\mathbf{s}}^{T}\right]^{T}$ is the same as that of $\boldsymbol{\varphi}_{2}$. When the error component in $\mathbf{G}_{2}$ is small enough to be neglected ( $\tilde{\mathbf{u}}_{i}$ sufficiently accurate), based on the WLS theory we have

$$
\operatorname{cov}(\hat{\boldsymbol{\theta}}) \simeq\left(\mathbf{G}_{2}^{T} \mathbf{W}_{2} \mathbf{G}_{2}\right)^{-1}=\left[\begin{array}{cc}
\tilde{\mathbf{X}} & \tilde{\mathbf{Y}} \\
\tilde{\mathbf{Y}} & \tilde{\mathbf{Z}}
\end{array}\right]^{-1}
$$


where

$$
\begin{aligned}
& \tilde{\mathbf{X}}=\mathbf{G}_{3}^{T} \mathbf{Q}_{r}^{-1} \mathbf{G}_{3}, \tilde{\mathbf{Y}}=\mathbf{G}_{3}^{T} \mathbf{Q}_{r}^{-1} \mathbf{G}_{4}, \tilde{\mathbf{Z}}=\mathbf{G}_{4}^{T} \mathbf{Q}_{r}^{-1} \mathbf{G}_{4}+\mathbf{Q}_{s}^{-1}, \\
& \mathbf{G}_{3}=\operatorname{diag}\left\{\mathbf{G}_{3,1}, \mathbf{G}_{3,2}, \cdots, \mathbf{G}_{3, K}\right\} \\
& \mathbf{G}_{3, i}=\mathbf{B}_{1, i}^{-1} \mathbf{G}_{1, i} \mathbf{B}_{2, i}^{-1} \mathbf{G}_{2, i}, \\
& \mathbf{G}_{4}=\left[\mathbf{G}_{4,1}^{T}, \mathbf{G}_{4,2}^{T}, \cdots, \mathbf{G}_{4, K}^{T}\right]^{T}, \mathbf{G}_{4, i}=-\mathbf{B}_{1, i}^{-1} \mathbf{D}_{i} .
\end{aligned}
$$

Following a procedure similar to that in Appendix $\mathrm{V}$ of [62], we can prove that when the noise is small compared to target range,

$$
\frac{\| \Delta \mathbf{s}_{j}||}{r_{j, i}^{o}} \simeq 0, \frac{\left|n_{j, i}\right|}{r_{j, i}^{o}} \simeq 0, i=1,2, \cdots, K, j=1,2, \cdots, M
$$

we have

$$
\mathbf{G}_{3} \simeq \frac{\partial \mathbf{r}^{o}}{\partial \mathbf{u}^{o}}, \quad \mathbf{G}_{4} \simeq \frac{\partial \mathbf{r}^{o}}{\partial \mathbf{s}^{o}}
$$

Putting (6.20) into (6.18) and comparing (6.17) with the CRLB given in Appendix A of $[20]$ yield

$$
\operatorname{cov}(\hat{\boldsymbol{\theta}}) \simeq \operatorname{CRLB}\left(\boldsymbol{\theta}^{o}\right)
$$

From the small noise analysis above, the proposed solution is able to attain the CRLB accuracy for both the source location and sensor position estimates.

\subsection{Simulation}

A total of 100 random localization geometries are used in the simulations. Each geometry has $K=2$ sources and $M=5$ sensors, where the sources and sensors are 
placed randomly with uniform distribution over a square area of $100 \times 100$ and $60 \times 60$ respectively. Fig. 6.2 shows the overlay of the 100 geometries.

The performance indices are the mean squared error (MSE) of the estimates computed by $\operatorname{mse}(\mathbf{u})=\frac{1}{K} \sum_{i=1}^{K}\left(\sum_{l=1}^{L} \hat{\mathbf{u}}_{i}^{(l)}-\mathbf{u}^{o} \|^{2} / L\right)$ and mse $(\mathbf{s})=\sum_{l=1}^{L}\left\|\hat{\mathbf{s}}^{(l)}-\mathbf{s}^{o}\right\|^{2} / L$, where $L$ is the number of ensemble runs, $\hat{\mathbf{u}}_{i}^{(l)}$ and $\hat{\mathbf{s}}^{(l)}$ are the $i$ th source location estimate and the sensor position estimates at ensemble $l$. Besides the proposed method, the sequential method [20] (estimation-refinement scheme) and the iterative MLE [6] are implemented for comparison. The sensor position estimates from [62] are also included. The approach in [62] is applied to obtain the hypothetical source locations for the proposed estimator. The same hypothetical source locations are used as the initial guesses for the MLE .

The covariance matrix of the TOA measurements (after multiplied with signal propagation speed square) is $\mathbf{Q}_{r}=\sigma_{r}^{2} \mathbf{I}$, where $\mathbf{I}$ is an identity matrix of size $M K$, $\sigma_{r}^{2}$ is the noise power which is fixed to $10^{-3}$ in the simulations. The covariance matrix of the sensor positions is $\mathbf{Q}_{s}=\sigma_{s}^{2} \mathbf{J}$, where $\mathbf{J}$ is a $N M \times N M$ diagonal matrix whose diagonal elements are uniformly distributed between 1 and 10 . We generate a different $\mathbf{J}$ for each localization geometry. $\sigma_{s}^{2}$ is a scaling proportion of the sensor position covariance matrix whose value varies between $10^{-2.25}$ and $10^{0.25}$. The number of ensemble runs $L$ is 1000 in each geometry and the results given are the average over the 100 geometries.

Fig. 6.3 gives the performance for source and sensor position estimates as the sensor position noise power increases. The sensor position noise power $\left(\boldsymbol{\sigma}_{\text {avg }}^{2}\right)$ in the $\mathrm{x}$ axis is trace $\left(\mathbf{Q}_{s}\right) /(N M)$ averaged over the 100 geometries. From the source location estimate results in Fig. 6.3(a), when $\boldsymbol{\sigma}_{\text {avg }}^{2}$ is not larger than $10^{-0.25}$, the 
proposed method, the sequential method and the MLE give similar results and attain the CRLB accuracy. For the sensor estimates in Fig. 6.3(b), the proposed method is always better than [62] by more than $1 \mathrm{~dB}$ when $\boldsymbol{\sigma}_{\text {avg }}^{2}$ is not larger than 1 . When $\boldsymbol{\sigma}_{\text {avg }}^{2}$ exceeds $10^{0.5}$, the proposed method is worse than [62] because of the joint estimation rather than the sensor position estimation only as in [62]. The proposed method also outperforms the sequential method when $\boldsymbol{\sigma}_{\text {avg }}^{2}$ is larger than 0.1. The MLE deviates from the CRLB slightly later than the proposed method but it requires iterations and higher computational cost.

The improvement of computation speed of the proposed method over the MLE (with an average of 3 iterations) is about a factor of two, measured using computation time in matlab for the simulations provided. The actual speed improvement could vary depending on implementations.

One purpose of refining the sensor positions is for better locating a newly appeared source. To demonstrate, we continue the simulation study as follows: for each of the 100 random geometries of two sources and five sensors, we add one new emitting source. After the positions of the two sources and five sensors are estimated, the refined sensor positions are used to locate the new source and the results are shown in Fig. 6.4. We observe that the CRLB of the new source location estimate is about $3 \mathrm{~dB}$ lower when using the refined sensor positions. The proposed method performs better than the sequential method in estimating the new source position. Interestingly enough, it yields comparable results with the MLE. 


\subsection{Concluding Remarks}

In this paper, we have developed an algebraic solution that jointly estimates the positions of multiple sources and sensors. The proposed method is able to achieve the CRLB performance for both the source and the sensor locations. The refined sensor positions can improve the localization of newly appeared sources subsequently. The good performance of the proposed estimator is shown analytically and supported by simulations. Compared to the sequential estimation-refinement technique, the proposed estimator provides better performance in sensor position estimates at higher noise level.
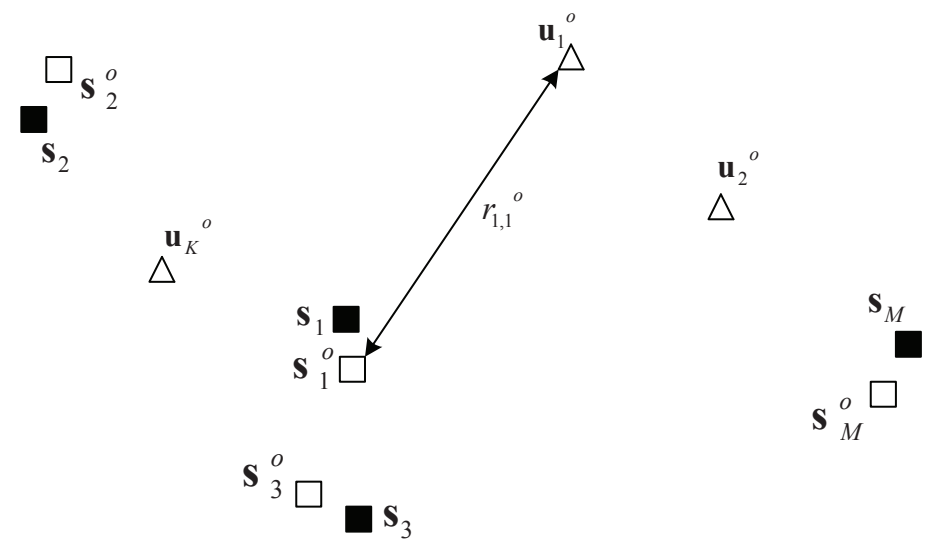

Figure 6.1: Localization of the sources at $\mathbf{u}_{i}^{o}$ using sensors at $\mathbf{s}_{j}$. Open squares denote the true sensor positions that are not known and closed squares are the available sensor positions that are erroneous. 


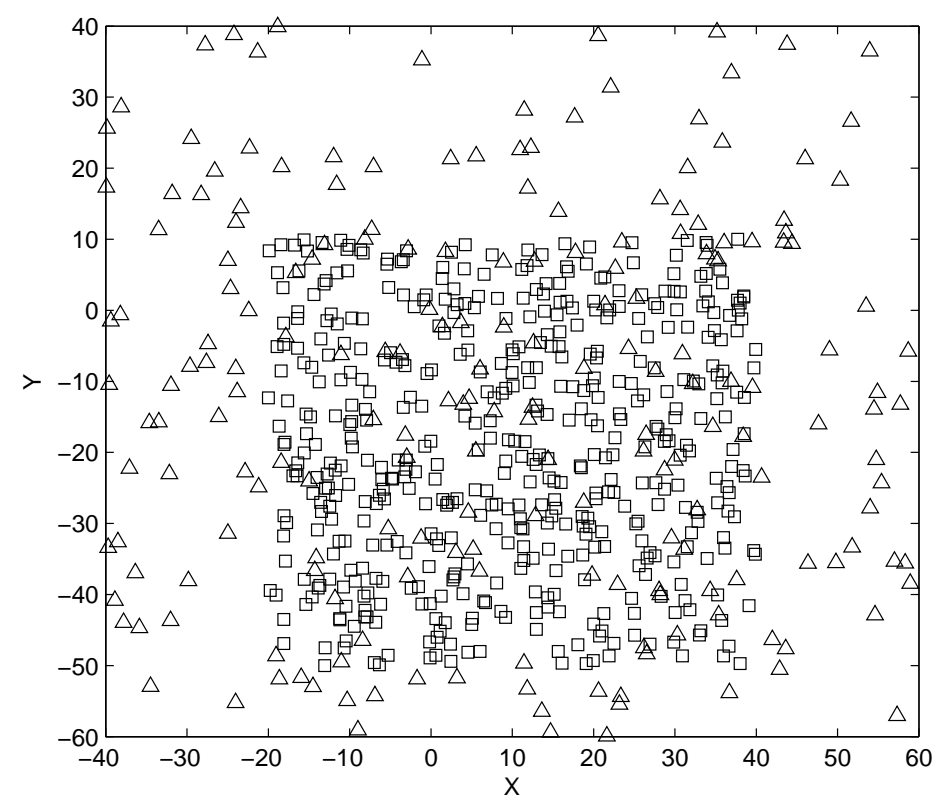

Figure 6.2: The 100 random localization geometries, upper triangles represent the sources and open squares denote the sensors.
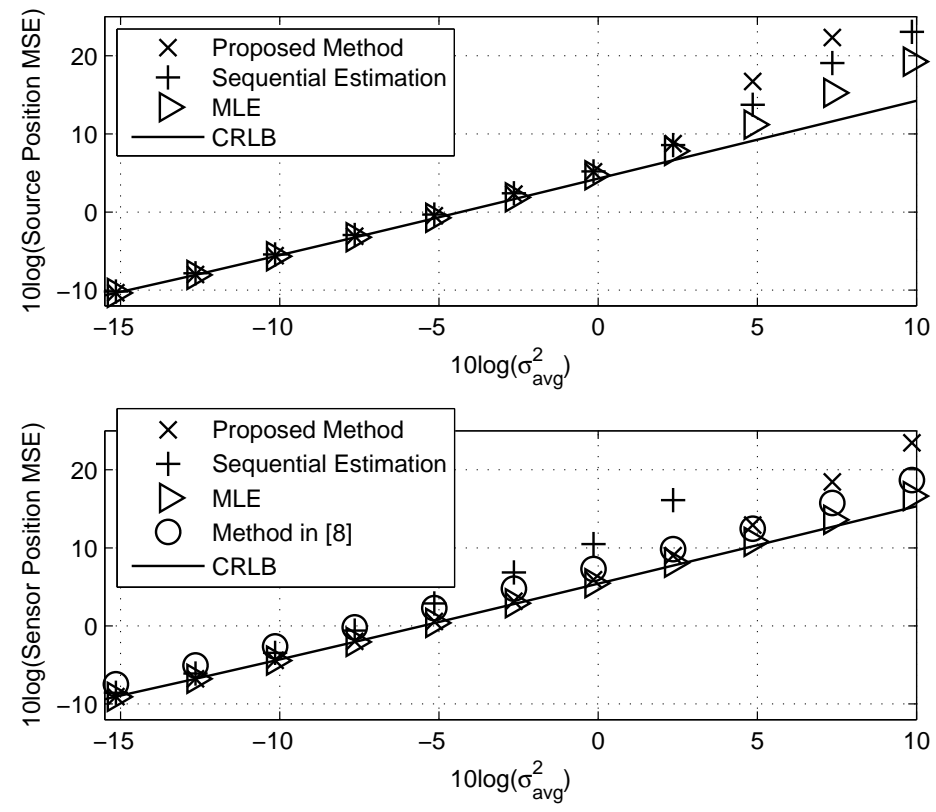

Figure 6.3: Comparison of the estimation accuracy when $\sigma_{r}^{2}$ is fixed to $10^{-3}$ and the sensor position noise power varies. (a) Results on source location estimates. (b) Results on sensor position estimates. 


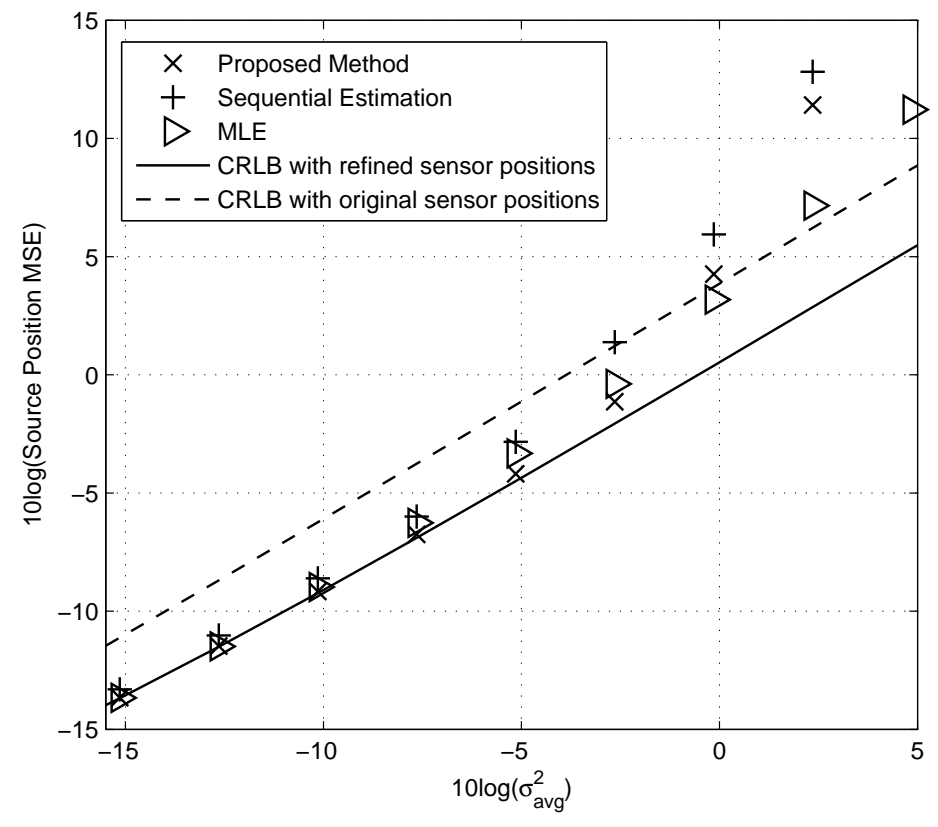

Figure 6.4: Estimation results of the new source location when $\sigma_{r}^{2}$ is fixed to $10^{-3}$ and the sensor position noise power varies. 


\title{
Chapter 7
}

\section{A Study on the Effects of Sensor}

\author{
Position Error and Calibration
}

\section{Emitter Placement for Source}

\section{Localization}

\subsection{Localization Problem}

The studies in Chapter 5 and Chapter 6 show that an estimator would require the use of the statistical knowledge of the sensor position errors in order to improve the source location estimate accuracy. It is common believe that ignoring the sensor position errors and pretending they are accurate will result in non-optimum performance. However, the research presented in this chapter shows that conditions exist under which one can simply assume the sensor position error is absent and yet achieving the 
optimum CRLB performance. In such cases, the placement of a calibration emitter is necessary to correct the sensor position errors.

We are interested to locate a stationary source using $M$ sensors as shown in Fig. 7.1. The unknown source location is dented by the column vector $\mathbf{u}^{o}$. The sensor positions when the measurements were acquired are $\mathbf{s}_{i}^{o}, i=1,2, \cdots, M$. However, $\mathbf{s}_{i}^{o}$ are not known and only the deviated sensor positions $\mathbf{s}_{i}$ are available to us. We shall represent the sensor position vector as

$$
\mathbf{s}=\left[\mathbf{s}_{1}^{T}, \mathbf{s}_{2}^{T}, \cdots . \mathbf{s}_{M}^{T}\right]^{T}=\mathbf{s}^{o}+\mathbf{n}_{s} .
$$

$\mathbf{n}_{s}$ is the sensor position noise vector and it is modeled as a zero-mean Gaussian random vector with covariance matrix $\mathbf{Q}_{s}=E\left[\mathbf{n}_{s} \mathbf{n}_{s}^{T}\right]$.

To locate the source, the sensor array generates a set of positioning variables, simply called measurements, based on the received signals from the source,

$$
\mathbf{m}=\mathbf{m}^{o}+\mathbf{n}_{m}
$$

where $\mathbf{m}^{o}$ is the true measurement vector and $\mathbf{n}_{m}$ is the noise. The measurements considered here are TOA, TDOA and AOA. The measurement noise is modeled as zero-mean Gaussian with covariance matrix $\mathbf{Q}_{m}=E\left[\mathbf{n}_{m} \mathbf{n}_{m}^{T}\right]$. We further assume that the sensor position noise and the measurement noise are uncorrelated so that $E\left[\mathbf{n}_{s} \mathbf{n}_{m}^{T}\right]=\mathbf{O}$

The localization accuracy is characterized by the CRLB which is the inverse of the FIM $[6]$. Defining the data vector as $\mathbf{x}=\left[\mathbf{m}^{T}, \mathbf{s}^{T}\right]^{T}$ and the unknown vector as 
$\boldsymbol{\theta}^{o}=\left[\mathbf{u}^{o T}, \mathbf{s}^{o T}\right]^{T}$, the FIM of $\boldsymbol{\theta}^{o}$ is

$$
\mathrm{FIM}=-E\left[\frac{\partial^{2} \ln p\left(\mathbf{x} ; \boldsymbol{\theta}^{o}\right)}{\partial \boldsymbol{\theta}^{o} \partial \boldsymbol{\theta}^{o T}}\right]=\left[\begin{array}{cc}
\mathbf{X} & \mathbf{Y} \\
\mathbf{Y}^{T} & \mathbf{Z}
\end{array}\right]
$$

where $p\left(\mathbf{x} ; \boldsymbol{\theta}^{o}\right)$ is the probability density function of $\mathbf{x}$ parameterized on $\boldsymbol{\theta}^{o}$. From the Gaussian density function,

$$
\begin{aligned}
& \mathbf{X}=\mathbf{G}_{u}^{T} \mathbf{Q}_{m}^{-1} \mathbf{G}_{u}, \mathbf{Y}=\mathbf{G}_{u}^{T} \mathbf{Q}_{m}^{-1} \mathbf{G}_{s} \\
& \mathbf{Z}=\mathbf{G}_{s}^{T} \mathbf{Q}_{m}^{-1} \mathbf{G}_{s}+\mathbf{Q}_{s}^{-1}
\end{aligned}
$$

In (7.4), $\mathbf{G}_{u}=\left(\frac{\partial \mathbf{m}^{o}}{\partial \mathbf{u}^{o}}\right)$ denotes the gradient of $\mathbf{m}^{o}$ with respect to $\mathbf{u}^{o}$ and $\mathbf{G}_{s}=\left(\frac{\partial \mathbf{m}^{o}}{\partial \mathbf{s}^{o}}\right)$ is that with respect to $\mathbf{s}^{o}$.

Invoking the block matrix inversion formula [6] gives the CRLB of $\mathbf{u}^{o}$ and $\mathbf{s}^{o}$ from the upper left and lower right blocks,

$$
\begin{aligned}
& \operatorname{CRLB}\left(\mathbf{u}^{o}\right)=\left(\mathbf{X}-\mathbf{Y} \mathbf{Z}^{-1} \mathbf{Y}^{T}\right)^{-1} \\
& \operatorname{CRLB}\left(\mathbf{s}^{o}\right)=\left(\mathbf{Z}-\mathbf{Y}^{T} \mathbf{X}^{-1} \mathbf{Y}\right)^{-1} .
\end{aligned}
$$

Applying the matrix inversion lemma [6] gives an alternative form of (7.5) as

$$
\operatorname{CRLB}\left(\mathbf{u}^{o}\right)=\mathbf{X}^{-1}+\mathbf{X}^{-1} \mathbf{Y C R L B}\left(\mathbf{s}^{o}\right) \mathbf{Y}^{T} \mathbf{X}^{-1}
$$

The first term on the right is the CRLB of the source location when there are no sensor position errors. The second term is the increase of the CRLB caused by the sensor position errors and it is proportional to the estimation accuracy of the sensor 
positions.

It appears from (7.7) that it is necessary to take into account the inaccuracy of the sensor positions, such as by estimating them together with the source position, in order to reach the CRLB accuracy. We would like to investigate if situations exist where taking the statistics of the sensor position errors when estimating the source location will not provide better result.

\subsection{Performance when Ignoring Sensor Position Errors}

The MLE is known to be asymptotic efficient in reaching the CRLB performance [6]. Let us denote the MLE for estimating a source location with exact sensor positions as $\mathrm{MLE}_{u}$ and that for estimating the source and sensor positions jointly as $\mathrm{MLE}_{u s}$. We shall evaluate the mean-square error matrix $\operatorname{MSE}$ defined as $\operatorname{MSE}(\mathbf{u})=E[(\mathbf{u}-$ $\left.\mathbf{u}^{o}\right)\left(\mathbf{u}-\mathbf{u}^{o}\right)^{T}$, where $\mathbf{u}$ is the source position estimate from $\mathrm{MLE}_{u}$ by pretending noisy sensor positions $\mathbf{s}_{i}$ as accurate. A contrast of the $\operatorname{MSE}(\mathbf{u})$ and the $\operatorname{CRLB}\left(\mathbf{u}^{o}\right)$, which is the asymptotic performance of $\mathrm{MLE}_{u s}$, provides the insight on how sensitive is the localization accuracy with respect to the sensor position errors.

The MSE for the special case of TDOA localization was examined in [13]. We generalize the derivations here and extend the results for an arbitrary measurement type. The development uses small error analysis up to linear noise term.

We shall use $\mathbf{f}(\mathbf{u})$ to denote the functional relationship of the measurement vector $\mathbf{m}$ in terms of $\mathbf{u}$ when using $\mathbf{s}$ as the sensor position vector. For example, the first element of $\mathbf{f}(\mathbf{u})$ is $\left\|\mathbf{u}-\mathbf{s}_{1}\right\|$ for TOA. Note that $\mathbf{f}\left(\mathbf{u}^{o}\right) \neq \mathbf{m}^{o}$ because $\mathbf{f}\left(\mathbf{u}^{o}\right)$ is computed 
using the noisy sensor positions and $\mathbf{m}^{o}$ is with respect to the true sensor positions.

Let us pretend $\mathbf{s}$ is the actual sensor positions and use $\mathrm{MLE}_{u}$ to estimate the source location. Under Gaussian noise the cost function of $\mathrm{MLE}_{u}$ is $\mathbf{e}_{f}^{T} \mathbf{Q}_{m}^{-1} \mathbf{e}_{f}$, where $\mathbf{e}_{f}=\mathbf{m}-\mathbf{f}(\mathbf{u}) . \mathbf{f}(\mathbf{u})$ is a nonlinear function of $\mathbf{u}$ and it is very difficult to minimize the cost function. One has to use iterative approach to obtain the location estimate.

The localization performance of $\mathrm{MLE}_{u}$ is examined by expanding $\mathbf{f}(\mathbf{u})$ using the Taylor-series at $\mathbf{u}=\mathbf{u}^{o}$ up to the linear term

$$
\mathbf{f}(\mathbf{u}) \simeq \mathbf{f}\left(\mathbf{u}^{o}\right)+\mathbf{F}\left(\mathbf{u}^{o}\right)\left(\mathbf{u}-\mathbf{u}^{o}\right)
$$

where $\mathbf{F}\left(\mathbf{u}^{o}\right)=\left.\frac{\partial \mathbf{f}(\mathbf{u})}{\partial \mathbf{u}}\right|_{\mathbf{u}^{o}}$. It can be approximated by $\mathbf{G}_{u}$ when the sensor position error is small and $\mathbf{u}$ is not far away from $\mathbf{u}^{o}$ because the effect of sensor position noise in $\mathbf{f}\left(\mathbf{u}^{o}\right)$ will be diminished after multiplying with $\left(\mathbf{u}-\mathbf{u}^{o}\right)$. Hence

$$
\mathbf{e}_{f} \simeq \mathbf{m}-\mathbf{f}\left(\mathbf{u}^{o}\right)-\mathbf{G}_{u}\left(\mathbf{u}-\mathbf{u}^{o}\right)
$$

The cost function becomes quadratic in $\mathbf{u}$ and it reaches the minimum value at

$$
\mathbf{u}-\mathbf{u}^{o} \simeq \mathbf{X}^{-1} \mathbf{G}_{u}^{T} \mathbf{Q}_{m}^{-1}\left[\mathbf{m}-\mathbf{f}\left(\mathbf{u}^{o}\right)\right]
$$

where $\mathbf{X}$ is defined in (7.4). $\mathbf{f}\left(\mathbf{u}^{o}\right)$ is computed using the inaccurate sensor positions. Expanding $\mathbf{f}\left(\mathbf{u}^{o}\right)$ at the true sensor positions and keeping up to the first order noise term give $\mathbf{f}\left(\mathbf{u}^{o}\right) \simeq \mathbf{m}^{o}+\mathbf{G}_{s} \mathbf{n}_{s}$. Hence

$$
\mathbf{m}-\mathbf{f}\left(\mathbf{u}^{o}\right) \simeq \mathbf{n}_{m}-\mathbf{G}_{s} \mathbf{n}_{s}
$$


where we have used the fact that $\left.\frac{\partial \mathbf{f}\left(\mathbf{u}^{o}\right)}{\partial \mathbf{s}}\right|_{\mathbf{s}=\mathbf{s}^{o}}=\mathbf{G}_{s}$.

Substituting (7.11) into (7.10), multiplying by its transpose and taking expectation yield

$$
\operatorname{MSE}(\mathbf{u})=\mathbf{X}^{-1}+\mathbf{X}^{-1} \mathbf{Y} \mathbf{Q}_{s} \mathbf{Y}^{T} \mathbf{X}^{-1}
$$

and $\mathbf{Y}$ is defined in (7.4). Recall that $\mathbf{X}^{-1}$ is the CRLB of $\mathbf{u}$ when there is no sensor position errors. The second term on the right is the decrease in accuracy resulted from the sensor position uncertainties.

\subsection{CRLB and MSE Comparison}

We would like to investigate if conditions exist that even though the statistics of the sensor position errors are taken into account, we will not be able to improve the source location estimate. Under such conditions, the characteristics of the CRLB will be investigated further.

\subsubsection{Conditions for Identical CRLB and MSE}

For simplicity let us define $\tilde{\mathbf{G}}_{u}=\mathbf{Q}_{m}^{-1 / 2} \mathbf{G}_{u}$ and $\tilde{\mathbf{G}}_{s}=\mathbf{Q}_{m}^{-1 / 2} \mathbf{G}_{s}$. (7.4) can be written as

$$
\mathbf{X}=\tilde{\mathbf{G}}_{u}^{T} \tilde{\mathbf{G}}_{u}, \quad \mathbf{Y}=\tilde{\mathbf{G}}_{u}^{T} \tilde{\mathbf{G}}_{s}, \quad \mathbf{Z}=\tilde{\mathbf{G}}_{s}^{T} \tilde{\mathbf{G}}_{s}+\mathbf{Q}_{s}^{-1} .
$$

The $\operatorname{CRLB}\left(\mathbf{u}^{o}\right)$ in (7.7) and the $\operatorname{MSE}(\mathbf{u})$ in (7.12) differ from each other in their second terms. Let us simplify the second term of (7.7). Putting (7.13) to the CRLB( $\left.\mathbf{s}^{o}\right)$ in 
(7.6) yields

$$
\operatorname{CRLB}\left(\mathbf{s}^{o}\right)^{-1}=\mathbf{Q}_{s}^{-1}+\tilde{\mathbf{G}}_{s}^{T} \tilde{\mathbf{G}}_{u}^{\perp} \tilde{\mathbf{G}}_{s}
$$

where $\tilde{\mathbf{G}}_{u}^{\perp}=\mathbf{I}-\tilde{\mathbf{G}}_{u}\left(\tilde{\mathbf{G}}_{u}^{T} \tilde{\mathbf{G}}_{u}\right)^{-1} \tilde{\mathbf{G}}_{u}^{T}$ is the orthogonal projection matrix of the subspace spanned by the columns of $\tilde{\mathbf{G}}_{u}$ and $\mathbf{I}$ is an identity matrix of appropriate size. Invoking the matrix inversion lemma [6] gives

$$
\operatorname{CRLB}\left(\mathbf{s}^{o}\right)=\mathbf{Q}_{s}-\mathbf{Q}_{s}\left[\left(\tilde{\mathbf{G}}_{s}^{T} \tilde{\mathbf{G}}_{u}^{\perp} \tilde{\mathbf{G}}_{s}\right) \mathbf{Q}_{s}+\mathbf{I}\right]^{-1} \tilde{\mathbf{G}}_{s}^{T} \tilde{\mathbf{G}}_{u}^{\perp} \tilde{\mathbf{G}}_{s} \mathbf{Q}_{s} .
$$

Hence upon using $\mathbf{Y}=\tilde{\mathbf{G}}_{u}^{T} \tilde{\mathbf{G}}_{s}$,

$$
\operatorname{CRLB}\left(\mathbf{s}^{o}\right) \mathbf{Y}^{T}=\mathbf{Q}_{s} \mathbf{Y}^{T}-\mathbf{Q}_{s}\left[\left(\tilde{\mathbf{G}}_{s}^{T} \tilde{\mathbf{G}}_{u}^{\perp} \tilde{\mathbf{G}}_{s}\right) \mathbf{Q}_{s}+\mathbf{I}\right]^{-1} \tilde{\mathbf{G}}_{s}^{T} \tilde{\mathbf{G}}_{u}^{\perp} \tilde{\mathbf{G}}_{s} \mathbf{Q}_{s} \tilde{\mathbf{G}}_{s}^{T} \tilde{\mathbf{G}}_{u}
$$

If there exists situation such that

$$
\tilde{\mathbf{G}}_{s} \mathbf{Q}_{s} \tilde{\mathbf{G}}_{s}^{T}=k \mathbf{I}
$$

where $k$ is a scalar constant, then the second term in (7.16) vanishes because $\tilde{\mathbf{G}}_{u}^{\perp} \tilde{\mathbf{G}}_{u}$ is zero. As a result

$$
\operatorname{CRLB}\left(\mathbf{s}^{o}\right) \mathbf{Y}^{T}=\mathbf{Q}_{s} \mathbf{Y}^{T}
$$

and (7.7) becomes

$$
\operatorname{CRLB}\left(\mathbf{u}^{o}\right)=\mathbf{X}^{-1}+\mathbf{X}^{-1} \mathbf{Y Q}_{s} \mathbf{Y}^{T} \mathbf{X}^{-1}=\operatorname{MSE}(\mathbf{u}) .
$$


Consequently, we conclude that when condition (7.17), or alternatively

$$
\mathbf{G}_{s} \mathbf{Q}_{s} \mathbf{G}_{s}^{T}=k \mathbf{Q}_{m}
$$

is satisfied, the CRLB will equal to the MSE.

\subsubsection{Increase in CRLB due to Sensor Position Errors}

Under the condition (7.20) and using (7.4),

$$
\mathbf{Y Q}_{s} \mathbf{Y}^{T}=\mathbf{G}_{u}^{T} \mathbf{Q}_{m}^{-1} \mathbf{G}_{s} \mathbf{Q}_{s} \mathbf{G}_{s}^{T} \mathbf{Q}_{m}^{-1} \mathbf{G}_{u}=k \mathbf{X}
$$

Putting (7.21) in the second term of (7.19) yields a very simple expression for the increase in CRLB due to sensor position errors:

$$
\Delta \operatorname{CRLB}\left(\mathbf{u}^{o}\right)=k \mathbf{X}^{-1} .
$$

We reach an interesting result that the increase in CRLB is a scalar multiple of $\mathbf{X}^{-1}$, the CRLB when the sensor position errors are absent. The value of $k$ depends on the specific measurement type used and sensor position noise powers which will be elaborated further in the subsequent section.

The condition (7.20) is not much useful in practice because it requires the exact knowledge of the measurement and sensor position noise covariance matrices. Most important, it is dependent on the source location to be estimated through the gradient matrix $\mathbf{G}_{s}$. However, geometry independent relation does exist. 


\subsection{Geometry Independent Conditions For Differ- ent Positionings}

We shall identify the geometry independent solutions to the condition (7.20) for the TOA, TDOA and AOA localizations.

\subsubsection{TOA}

Let

$$
r_{i}^{o}=\left\|\mathbf{u}^{o}-\mathbf{s}_{i}^{o}\right\|
$$

be the true range between the source and sensor $i$, where $\|\cdot\|$ denotes the Euclidean norm. After multiplying the TOAs with the signal propagation speed, the measurement vector $\mathbf{m}$ is $\overline{\mathbf{m}}=\mathbf{r}^{o}+\mathbf{n}_{m}$, where $\mathbf{r}^{o}=\left[r_{1}^{o}, r_{2}^{o}, \cdots, r_{M}^{o}\right]^{T}$.

Let

$$
\mathbf{q}_{i}=\left(\mathbf{u}^{o}-\mathbf{s}_{i}^{o}\right) / r_{i}^{o}
$$

be the unit vector pointing from $\mathbf{s}_{i}^{o}$ to $\mathbf{u}^{o}$. The $i$ th rows, $i=1,2, \cdots, M$ of $\mathbf{G}_{u}$ and $\mathbf{G}_{s}$ are

$$
\begin{gathered}
\overline{\mathbf{G}}_{u}(i,:)=\left(\frac{\partial r_{i}^{o}}{\partial \mathbf{u}^{o}}\right)^{T}=\mathbf{q}_{i}^{T} \\
\overline{\mathbf{G}}_{s}(i,:)=\left(\frac{\partial r_{i}^{o}}{\partial \mathbf{s}^{o}}\right)^{T}=\left[\mathbf{0}_{N(i-1) \times 1}^{T},-\mathbf{q}_{i}^{T}, \mathbf{0}_{N(M-i) \times 1}^{T}\right]
\end{gathered}
$$

where $N$ is the dimension of localization. We have called the $\mathbf{m}, \mathbf{G}_{u}$ and $\mathbf{G}_{s}$ in the TOA case as $\overline{\mathbf{m}}, \overline{\mathbf{G}}_{u}$ and $\overline{\mathbf{G}}_{s}$ to distinguish them from those of TDOA and AOA cases. 
It is straightforward to validate that $\overline{\mathbf{G}}_{s}$ satisfies the relation

$$
\overline{\mathbf{G}}_{s} \overline{\mathbf{G}}_{s}^{T}=\mathbf{I}_{M}
$$

where $\mathbf{I}_{M}$ is an identity matrix of size $M$.

A careful look at (7.20) together with (7.26) shows that the geometry independent solution to $(7.20)$ is:

$$
\mathbf{Q}_{m}=k \boldsymbol{\Sigma}, \quad \mathbf{Q}_{s}=\boldsymbol{\Sigma} \otimes \mathbf{I}_{N}
$$

where

$$
\boldsymbol{\Sigma}=\operatorname{diag}\left\{\sigma_{1}^{2}, \sigma_{2}^{2}, \cdots, \sigma_{M}^{2}\right\}
$$

is a diagonal matrix containing the average noise powers in the coordinates of each sensor and $\otimes$ is the Kronecker product. In particular, if the noise powers of the $M$ TOA measurements are the same, we simply have

$$
\mathbf{Q}_{m}=\sigma_{r}^{2} \mathbf{I}_{M} \quad, \quad \mathbf{Q}_{s}=\sigma_{s}^{2} \mathbf{I}_{N M}
$$

In other words, we arrive at the interesting result that if the measurement noise and sensor position covariance matrices are proportional to an identity matrix, there is no need to take the sensor position errors into account in the estimation regardless of what the localization geometry is. IID TOA measurement noise occurs when the source is distant or in message exchange based sensor networks [64].

For the special solution $(7.29), k$ is

$$
k=\frac{\sigma_{s}^{2}}{\sigma_{r}^{2}}
$$


and the increase of CRLB in (7.22) becomes, upon using the definition of $\mathbf{X}$ in (7.4),

$$
\Delta \operatorname{CRLB}\left(\mathbf{u}^{o}\right)=\sigma_{s}^{2}\left(\overline{\mathbf{G}}_{u}^{T} \overline{\mathbf{G}}_{u}\right)^{-1} .
$$

It is directly proportional to the sensor position noise power.

\subsubsection{TDOA}

When using $\mathbf{s}_{1}^{o}$ as the reference sensor, the TDOA measurement vector, after multiplying with the signal propagation speed, is related to that of TOA by

$$
\mathbf{m}=\mathbf{H} \overline{\mathbf{m}} .
$$

The matrix $\mathbf{H}$ is defined as

$$
\mathbf{H}=\left[\begin{array}{ll}
-\mathbf{1}_{M-1} & \mathbf{I}_{M-1}
\end{array}\right]
$$

where $\mathbf{1}_{M-1}$ is the length ( $\left.M-1\right)$ column vector of unity. From (7.32), $\mathbf{G}_{u}$ and $\mathbf{G}_{s}$ are

$$
\mathbf{G}_{u}=\left(\frac{\partial \mathbf{m}^{o}}{\partial \mathbf{u}^{o}}\right)=\mathbf{H} \overline{\mathbf{G}}_{u} \quad, \quad \mathbf{G}_{s}=\left(\frac{\partial \mathbf{m}^{o}}{\partial \mathbf{s}^{o}}\right)=\mathbf{H} \overline{\mathbf{G}}_{s}
$$

and $\overline{\mathbf{G}}_{u}$ and $\overline{\mathbf{G}}_{s}$ are defined in (7.25). We have from (7.26) that

$$
\mathbf{G}_{s} \mathbf{G}_{s}^{T}=\mathbf{H H}^{T}
$$

The geometry independent solution of $\mathbf{Q}_{m}$ and $\mathbf{Q}_{s}$ that satisfies (7.20) is

$$
\mathbf{Q}_{m}=k \mathbf{H} \mathbf{\Sigma} \mathbf{H}^{T} \quad, \quad \mathbf{Q}_{s}=\boldsymbol{\Sigma} \otimes \mathbf{I}_{N}
$$


where $\boldsymbol{\Sigma}$ is defined in (7.28). In the special case of $\boldsymbol{\Sigma}=\sigma_{r}^{2} \mathbf{I}_{M}$, we have

$$
\mathbf{Q}_{m}=\sigma_{r}^{2} \mathbf{H H}^{T}, \mathbf{Q}_{s}=\sigma_{s}^{2} \mathbf{I}_{N M}
$$

This form of $\mathbf{Q}_{m}$ often appears in distant source estimation [13,22] and in radar [65].

The corresponding factor $k$ has the same value in (7.30) and (7.22) becomes, after using the definition of $\mathbf{X}$ in (7.4),

$$
\Delta \operatorname{CRLB}\left(\mathbf{u}^{o}\right)=\sigma_{s}^{2}\left[\overline{\mathbf{G}}_{u}^{T}\left(\mathbf{I}_{M}-\frac{\mathbf{1}_{M} \mathbf{1}_{M}^{T}}{M}\right) \overline{\mathbf{G}}_{u}\right]^{-1}
$$

where $\mathbf{H}^{T}\left(\mathbf{H} \mathbf{H}^{T}\right)^{-1} \mathbf{H}=\left(\mathbf{I}_{M}-\mathbf{1}_{M} \mathbf{1}_{M}^{T} / M\right)$ has been used. The matrix $\mathbf{1}_{M} \mathbf{1}_{M}^{T}$ is positive semi-definite. Hence the increase in CRLB for TDOA will be at least as much as that for TOA.

\subsubsection{AOA}

Unlike TOA and TDOA where distances are used to estimate the source location, AOA utilizes the bearing between the source and sensors. AOA positioning is mostly used for 2D localization. The true bearing of the source with respect to sensor $i$ is

$$
b_{i}^{o}=\tan ^{-1} \frac{y^{o}-y_{i}^{o}}{x^{o}-x_{i}^{o}}, \quad i=1,2, \cdots, M
$$

The measurement vector is $\mathbf{m}=\mathbf{b}^{o}+\mathbf{n}_{m}$, where $\mathbf{b}^{o}=\left[b_{1}^{o}, b_{2}^{o}, \cdots, b_{M}^{o}\right]^{T}$. 
Using (7.39), we have

$$
\begin{aligned}
\left(\frac{\partial b_{i}^{o}}{\partial \mathbf{u}^{o}}\right)^{T} & =\left(\mathbf{T} \mathbf{q}_{i}\right)^{T} / r_{i}^{o}, \\
\left(\frac{\partial b_{i}^{o}}{\partial \mathbf{s}^{o}}\right)^{T} & =\left[\mathbf{0}_{2(i-1) \times 1}^{T},-\left(\mathbf{T q}_{i}\right)^{T} / r_{i}^{o}, \mathbf{0}_{2(M-i) \times 1}^{T}\right]
\end{aligned}
$$

and they can be expressed in terms of $\overline{\mathbf{G}}_{s}$ and $\overline{\mathbf{G}}_{u}$ as

$$
\mathbf{G}_{u}=\mathbf{R} \overline{\mathbf{G}}_{u} \mathbf{T}, \quad \mathbf{G}_{s}=\mathbf{R} \overline{\mathbf{G}}_{s}\left(\mathbf{I}_{M} \otimes \mathbf{T}\right)
$$

where

$$
\mathbf{T}=\left[\begin{array}{cc}
0 & 1 \\
-1 & 0
\end{array}\right] \quad, \quad \mathbf{R}=\operatorname{diag}\left\{\frac{1}{r_{1}^{o}}, \frac{1}{r_{2}^{o}}, \cdots, \frac{1}{r_{M}^{o}}\right\}
$$

Note that we have the equality $\mathbf{T} \mathbf{T}^{T}=\mathbf{I}_{2}$. Using the same form of $\mathbf{Q}_{s}$ as in TOA and TDOA, the solution to $(7.20)$ is

$$
\mathbf{Q}_{m}=k \mathbf{R} \mathbf{R} \mathbf{R}, \quad \mathbf{Q}_{s}=\mathbf{\Sigma} \otimes \mathbf{I}_{N}
$$

and $\boldsymbol{\Sigma}$ is defined in (7.28). In the special case of $\boldsymbol{\Sigma}=\sigma_{r}^{2} \mathbf{I}_{M}$,

$$
\mathbf{Q}_{m}=\sigma_{r}^{2} \mathbf{R}^{2}, \quad \mathbf{Q}_{s}=\sigma_{s}^{2} \mathbf{I}_{N M}
$$

Note that $\mathbf{Q}_{m}$ reduces back to scalar multiple of identity when the source is distant. AOA measurement noise is often independent because the AOAs come from different sensors of multiple elements.

The factor $k$ is again given by (7.30) and the increase of CRLB from (7.22) be- 
comes, after using the definition of $\mathbf{X}$ in (7.4),

$$
\Delta \operatorname{CRLB}\left(\mathbf{u}^{o}\right)=\sigma_{s}^{2} \mathbf{T}^{T}\left(\overline{\mathbf{G}}_{u}^{T} \overline{\mathbf{G}}_{u}\right)^{-1} \mathbf{T} .
$$

The condition (7.20) is geometry dependent in general. We have established geometry independent particular solutions (7.29) for TOA, (7.37) for TDOA and (7.44) for AOA ((7.44) is range dependent to be exact). They all require $\mathbf{Q}_{s}$ to be proportional to identity. Although this is not the case for the self-localized sensor nodes, this form of sensor position errors occurs in UWB localization [66] and beacon positions $[15,64]$.

\subsection{Optimum Calibrator Position}

Taking the statistical property of the sensor position errors into account cannot reduce the degradation in source localization accuracy if the condition (7.20) is satisfied. In such a situation or for the purpose of achieving better performance, we will need to deploy a calibration emitter whose position is known exactly to limit the damage caused by the sensor position uncertainties [22].

When a calibration source is available for deployment, we would like to decide where to place the calibration source so as to maximize the performance gain. This section derives the optimum calibration source position that yields the FIM whose difference with those from all other calibration positions to be positive definite (PD). While the solution may be of theoretical interest only, the insights gained from the derivations lead to the development of a practical criterion for the calibration emitter placement.

Let $\mathbf{c}$ be the Cartesian coordinate position of the calibration source to be deter- 
mined. The measurements from the calibration emitter to the sensors are

$$
\mathbf{m}_{c}=\mathbf{m}_{c}^{o}+\mathbf{n}_{c}
$$

where $\mathbf{m}_{c}^{o}$ is the true value and $\mathbf{n}_{c}$ is the calibration measurement noise vector. $\mathbf{n}_{c}$ follows zero-mean Gaussian distribution with covariance matrix $\mathbf{Q}_{c}$. To simplify the development, we shall assume $\mathbf{n}_{c}$ is independent of $\mathbf{n}_{m}$ and $\mathbf{n}_{s}$.

Starting from the composite data vector $\left[\mathbf{m}^{T}, \mathbf{m}_{c}^{T}, \mathbf{s}^{T}\right]^{T}$ and its probability density function, we can obtain, using the same steps as in Section II, the FIM of $\mathbf{u}^{o}$

$$
\operatorname{FIM}\left(\mathbf{u}^{o}\right)=\mathbf{X}-\mathbf{Y Z}_{c}^{-1} \mathbf{Y}^{T}
$$

$\mathbf{X}$ and $\mathbf{Y}$ are defined in (7.4), and $\mathbf{Z}_{c}$ is

$$
\mathbf{Z}_{c}=\mathbf{G}_{s}^{T} \mathbf{Q}_{m}^{-1} \mathbf{G}_{s}+\mathbf{G}_{c}^{T} \mathbf{Q}_{c}^{-1} \mathbf{G}_{c}+\mathbf{Q}_{s}^{-1}
$$

where $\mathbf{G}_{c}=\partial \mathbf{m}_{c}^{o} / \partial \mathbf{s}^{o}$ is the gradient of $\mathbf{m}_{c}^{o}$ with respect to $\mathbf{s}^{o}$.

We shall derive below the optimum value of $\mathbf{c}$ that makes $\operatorname{FIM}\left(\mathbf{u}^{o}\right)$ as large as possible in the PD sense for TOA, TDOA and AOA localizations.

\subsubsection{TOA}

In this case, $\mathbf{G}_{u}$ and $\mathbf{G}_{s}$ in (7.4) are equal to $\overline{\mathbf{G}}_{u}$ and $\overline{\mathbf{G}}_{s}$ given in (7.25a) and (7.25b). The matrix $\mathbf{G}_{c}$ is equal to $\overline{\mathbf{G}}_{c}$ whose $i$-th row is

$$
\overline{\mathbf{G}}_{c}(i,:)=\left[\mathbf{0}_{N(i-1) \times 1}^{T},-\boldsymbol{\gamma}_{i}^{T}, \mathbf{0}_{N(M-i) \times 1}^{T}\right]
$$


where

$$
\gamma_{i}=\left(\mathbf{c}-\mathbf{s}_{i}\right) /\left\|\mathbf{c}-\mathbf{s}_{i}\right\|
$$

is a unit vector from $\mathbf{s}_{i}$ to $\mathbf{c}$.

The optimization problem is quite challenging and not straightforward. By representing the columns of $\overline{\mathbf{G}}_{c}^{T}$ in the basis formed by the columns of $\overline{\mathbf{G}}_{s}^{T}$ and its orthogonal complement $\overline{\mathbf{G}}_{s}^{\perp T}$, Appendix A shows that (7.47) can be expressed as

$$
\operatorname{FIM}\left(\mathbf{u}^{o}\right)=\overline{\mathbf{G}}_{u}^{T}\left(\mathbf{Q}_{m}+\tilde{\mathbf{Q}}_{c}\right)^{-1} \overline{\mathbf{G}}_{u}
$$

where $\tilde{\mathbf{Q}}_{c}$ is defined in (7.78) and it is dependent on the calibration emitter position $\mathbf{c}$. Note that (7.51) is in the same form as $\mathbf{X}$ in (7.4), the FIM when sensor position errors are absent. Hence we can consider $\left(\mathbf{Q}_{m}+\tilde{\mathbf{Q}}_{c}\right)$ as the equivalent measurement noise covariance matrix when calibration emitter is available to reduce the sensor position uncertainties. The optimum calibration emitter placement problem is to reduce $\tilde{\mathbf{Q}}_{c}$ as much as possible in the PD sense as described below (7.80).

The optimization problem is not easy to solve in general. We shall consider a few special cases that yield easy and meaningful solutions.

$\mathbf{Q}_{s}=\sigma_{s}^{2} \mathbf{I}$

When $\mathbf{Q}_{s}$ is proportional to an identity matrix, Appendix B shows that the minimum value of $\tilde{\mathbf{Q}}_{c}$ in the $\mathrm{PD}$ sense is

$$
\tilde{\mathbf{Q}}_{c}^{o}=\left(\frac{1}{\sigma_{s}^{2}} \mathbf{I}+\mathbf{Q}_{c}^{-1}\right)^{-1}
$$


when the calibration emitter is placed at

$$
\mathbf{c}^{o}=\mathbf{u}^{o}
$$

The optimum calibrator position is at the unknown source location.

$\mathbf{Q}_{s} \ll \mathbf{Q}_{c}$

If $\mathbf{Q}_{s}$ is much smaller than $\mathbf{Q}_{c}$ in the sense that $\mathbf{Q}_{c}-h \mathbf{Q}_{s}$ remains $\mathrm{PD}$ for some large scalar value $h$, Appendix B deduces that the optimum calibration emitter position is the same as (7.53). In the extreme case as $\mathbf{Q}_{s} \rightarrow \mathbf{O}, \operatorname{FIM}\left(\mathbf{u}^{o}\right)$ reduces back to the one when the sensor positions are accurate.

\section{$\mathbf{Q}_{s} \gg \mathbf{Q}_{c}$}

In this case of very large sensor position uncertainties, Appendix B derives that the optimum calibration emitter position is also given by (7.53) and the corresponding FIM is

$$
\operatorname{FIM}\left(\mathbf{u}^{o}\right)=\overline{\mathbf{G}}_{u}^{T}\left(\mathbf{Q}_{m}+\mathbf{Q}_{c}\right)^{-1} \overline{\mathbf{G}}_{u}
$$

It should be noted that (7.53) is the optimum calibration emitter position for the three special cases considered above. It may not be given by (7.53) for other forms of $\mathbf{Q}_{s}$. 


\subsubsection{TDOA}

The gradient matrices $\mathbf{G}_{u}$ and $\mathbf{G}_{s}$ are related to those of TOA through (7.34). The same relationship occurs for $\mathbf{G}_{c}$ as well:

$$
\mathbf{G}_{c}=\mathbf{H} \overline{\mathbf{G}}_{c} .
$$

The covariance matrices $\mathbf{Q}_{m}$ and $\mathbf{Q}_{c}$ are of size $(M-1)$ in TDOA positioning. Using (7.34) and (7.55), (7.4) and (7.48) can be written as

$$
\begin{aligned}
& \mathbf{X}=\overline{\mathbf{G}}_{u}^{T}\left(\mathbf{H}^{T} \mathbf{Q}_{m}^{-1} \mathbf{H}\right) \overline{\mathbf{G}}_{u}, \mathbf{Y}=\overline{\mathbf{G}}_{u}^{T}\left(\mathbf{H}^{T} \mathbf{Q}_{m}^{-1} \mathbf{H}\right) \overline{\mathbf{G}}_{s}, \\
& \mathbf{Z}_{c}=\overline{\mathbf{G}}_{s}^{T}\left(\mathbf{H}^{T} \mathbf{Q}_{m}^{-1} \mathbf{H}\right) \overline{\mathbf{G}}_{s}+\overline{\mathbf{G}}_{c}^{T}\left(\mathbf{H}^{T} \mathbf{Q}_{c}^{-1} \mathbf{H}\right) \overline{\mathbf{G}}_{c}+\mathbf{Q}_{s}^{-1} .
\end{aligned}
$$

They are the same as those in the TOA case when $\mathbf{Q}_{m}^{-1}$ and $\mathbf{Q}_{c}^{-1}$ for TOA are replaced by

$$
\begin{aligned}
\mathbf{Q}_{m, T O A}^{-1} & =\mathbf{H}^{T} \mathbf{Q}_{m, T D O A}^{-1} \mathbf{H} \\
\mathbf{Q}_{c, T O A}^{-1} & =\mathbf{H}^{T} \mathbf{Q}_{c, T D O A}^{-1} \mathbf{H} .
\end{aligned}
$$

The results in Section 7.5.1 are valid for TDOA, as long as we made the substitution (7.57). Note that the matrix inversion lemma is needed in (7.80) and (7.87) when using the substitution (7.57). The optimum calibration source position remains to be given by (7.83) for the three special cases of $\mathbf{Q}_{s}$ in Section 7.5.1. 


\subsubsection{AOA}

The gradient matrices for the AOA case is related to those of the TOA case through (7.41) and

$$
\mathbf{G}_{c}=\mathbf{R} \overline{\mathbf{G}}_{c} \mathbf{T} .
$$

After substituting them into (7.47) and (7.48), the FIM for the AOA case is

$$
\operatorname{FIM}\left(\mathbf{u}^{o}\right)_{A O A}=\mathbf{T}^{T} \operatorname{FIM}\left(\mathbf{u}^{o}\right)_{T O A} \mathbf{T},
$$

where $\operatorname{FIM}\left(\mathbf{u}^{o}\right)_{T O A}$ is given by (7.80), (7.78) and (7.73), with $\mathbf{Q}_{m}$ and $\mathbf{Q}_{c}$ there replaced by

$$
\mathbf{R}^{-1} \mathbf{Q}_{m} \mathbf{R}^{-1}, \mathbf{R}^{-1} \mathbf{Q}_{c} \mathbf{R}^{-1}
$$

The matrix $\mathbf{T}$ is unitary. The results and conclusions from TOA are valid for AOA as well, where the optimum sensor position is (7.53) for the three specific cases of $\mathbf{Q}_{s}$ in Section 7.5.1.

\subsubsection{Suboptimum Calibration Emitter Position}

The investigation in Section 7.5.1-3 indicates that under the three specific forms of $\mathbf{Q}_{s}$, the optimum calibration emitter position is at the source position. It is not possible to deploy the calibration emitter at the source position because it is not known. Even if we know roughly where the source is, it may not be practical to place a calibration emitter nearby. We are interested to determine if there is any suboptimum calibration position.

The derivations in Appendices A and B provide the insight that it is preferable 
to allocate the calibration emitter to a position such that $\mathbf{A}$ is close to unity. $\mathbf{A}$ is a diagonal matrix defined in (7.65) and (7.69) where each diagonal element is the proportion of the unity vector between the calibration emitter and a sensor to the unity vector between the unknown source and the same sensor.

Premultiplying (7.68) by $\overline{\mathbf{G}}_{s}$ and using (7.25b), (7.26) and (7.49) yield

$$
\mathbf{A}=\overline{\mathbf{G}}_{s} \overline{\mathbf{G}}_{c}^{T}=\operatorname{diag}\left\{\mathbf{q}_{1}^{T} \boldsymbol{\gamma}_{1}, \mathbf{q}_{2}^{T} \boldsymbol{\gamma}_{2}, \ldots, \mathbf{q}_{M}^{T} \boldsymbol{\gamma}_{M}\right\}
$$

where $\mathbf{q}_{i}$ and $\boldsymbol{\gamma}_{i}$ are defined in (7.24) and (7.50). $\mathbf{A}$ appears as a pair in $\tilde{\mathbf{Q}}_{c}$ shown in (7.78) under the three specific cases of $\mathbf{Q}_{s}$ in Section 7.5.1 and the signs of the elements in $\mathbf{A}$ are irrelevant. Thus, we propose the criterion

$$
J=1-\frac{1}{M} \operatorname{trace}\left(\mathbf{A}^{2}\right)
$$

for minimization to obtain the calibration source position.

Computing (7.62) requires the true source and sensor positions that are not known. We shall apply $\mathrm{MLE}_{u s}$ to obtain the initial source and sensor locations, from which (7.62) can be evaluated for a given calibration emitter position. The simulation results in the next Section indicate suboptimum calibration position exists that could be far from $\mathbf{u}^{o}$.

In practice, many calibration emitters may be deployed in different locations that cover a large geographic area. Normally the calibration emitters do not send out any signals for various reasons such as reducing interferences or for security purposes, and they only do so as needed. When we are ready to locate an unknown source, the application of (7.62) at different calibration emitter locations can be evaluated. The 
one giving the smallest $J$ value will be activated to send out calibration signals for improving the source localization performance.

Other than the proposed $J$ criterion, the trace of the CRLB of the source location estimate can also be used as a criterion to determine the position of the calibration emitter. As the next Section shows, however, it requires much more computation to obtain the CRLB value than the proposed $J$ value. The proposed $J$ criterion is more efficient than the CRLB criterion.

\subsection{Simulation}

There are two sets of simulations. The first is for the validation of the relationships on the sensor position and measurement noise covariance matrices from which it is not necessary to take the sensor position errors into account to reach the CRLB performance. The second is for the placement of calibration emitter.

The simulation uses $M=6$ sensors to locate a source in 3-Dimensions for TOA and TDOA and in 2-Dimensions for AOA. The geometries for all three positionings are common and the $\mathrm{z}$ coordinates are set to zero for the AOA case. A total of 250 geometries are created randomly, where the sensors are within a cube with edge length of 200 units and the source is within a cube with edge length of 1000 units. The $\mathrm{x}$, $\mathrm{y}$ and $\mathrm{z}$ coordinates of the sensors and the source are uniformly distributed and are independent. The number of ensemble runs is 1000 for each of the random geometries and the mean-square localization error (mse) results presented are the average over the 250 geometries. The MLE with Gauss-Newton iterative implementation is used to estimate the source location in each ensemble run. The initial guesses of the source 
location and the sensor positions are randomly generated according to two Gaussian distributions whose means are the true values of the source and sensor positions, and the corresponding covariances are four times their CRLBs where only the diagonal elements are kept.

We shall use the following notations to represent the noise powers. The TOA (range) noise power is $\sigma_{r}^{2}=\operatorname{trace}\left(\mathbf{Q}_{m}\right) / M$, the TDOA (range difference) noise power is $\sigma_{d}^{2}=\operatorname{trace}\left(\mathbf{Q}_{m}\right) /(M-1)$, the AOA noise power is $\sigma_{a}^{2}=\operatorname{trace}\left(\mathbf{Q}_{m}\right) / M$ and the sensor position noise power is $\sigma_{s}^{2}=\operatorname{trace}\left(\mathbf{Q}_{s}\right) / N M$, where $N$ is the dimension of localization (3 for TOA and TDOA, and 2 for AOA).

\subsubsection{Special Relationships between $\mathrm{Q}_{s}$ and $\mathrm{Q}_{m}$}

To verify the relation (7.20), we generate randomly PD $\mathbf{Q}_{s}$ and use (7.20) to obtain $\mathbf{Q}_{m}$ for creating the measurement noise. $\mathbf{Q}_{s}$ and hence $\mathbf{Q}_{m}$ are different for each geometry but held fixed during the ensemble runs. Please note that we generate $\mathbf{Q}_{s}$ and $\mathbf{Q}_{m}$ in this manner for validation of (7.20) only. In practice, $\mathbf{Q}_{s}$ and $\mathbf{Q}_{m}$ are determined by the localization scenario and cannot be chosen.

The lower sets of curves in Figs. 7.2-7.4 give the results for TOA, TDOA and AOA positionings as the sensor position noise power increases. The noise settings are $\sigma_{r}^{2}=10^{-4}, \sigma_{d}^{2}=10^{-4}$ and $\sigma_{a}^{2}=10^{-4}$. In the figures, the circle symbol represents the estimation accuracy when applying $\mathrm{MLE}_{u s}$ that jointly estimates the source and sensor positions and the cross symbol denotes the performance when applying $\mathrm{MLE}_{u}$ that pretends the sensor positions are correct. Also shown are the CRLBs. It is clear that the performance of the two estimators overlaps with each other and meets the CRLB. Joint estimation provides better behavior in the case of TOA and AOA at 
large sensor position noise power when the thresholding effect starts to take place. It is interesting that the thresholding behaviors for the two estimators are very similar for the TDOA case which starts after $\sigma_{s}^{2}>0.1$.

When we keep $\sigma_{s}^{2}$ to $10^{-1}$ and vary the measurement noise powers, the performance is depicted in the lower sets of curves in Figs. 7.5-7.7. The simulation results confirm very well that under (7.20), taking the sensor position errors into account is not necessary and does not improve performance unless the sensor position noise power is large.

We also did the simulation for IID sensor position noise with the measurement covariance matrices given by (7.29), (7.37) for TOA and TDOA. They satisfy (7.20) and are geometry independent. For AOA, we approximate (7.44) by setting $\mathbf{Q}_{m}=$ $\sigma_{a}^{2} \mathbf{I}$ to obtain geometry independent relation. These forms of sensor position and measurement noise covariance matrices occur in many practical applications $[15,18$, 64,66-68]. The results are shown in the upper sets of curves in Figs 7.2-7.7. For Figs. 7.2-7.4, we used the same settings as before. For Figs. 7.5-7.7, $\sigma_{s}^{2}$ were set to 1 for TOA, 0.01 for TDOA and 0.1 for AOA to make the performance variations apparent. The observations and conclusions are essentially the same as those for the case of geometry dependent noise covariance matrices.

\subsubsection{Placement of Calibration Emitter}

To gain some understandings of the calibration emitter position on the source localization accuracy, we generate the theoretical mse for 2-D localization using TOA as an example in Fig. 7.8, where $\mathbf{Q}_{s}$ is set proportional to identity, the true sensor locations are shown as circles and the source location is marked as triangle. The 
theoretical mse is the trace of the inverse of the source location FIM in (7.47) at a given calibration emitter position defined by the $\mathrm{x}$ and y coordinates. Darker (bluer) level corresponds to smaller mse. Note that the y coordinate range is much smaller than that of the $\mathrm{x}$ coordinate.

The smallest mse occurs at the source location, indicating that this is the optimum location for the calibration source as anticipated by the theory. An interesting observation is that the theoretical mse does not increase monotonically as the calibration emitter position moves away from the unknown source location. Indeed, at the opposite side of the source with respect to the sensors, lower mse is also observed. It implies that placing a calibration emitter close to the unknown source is not necessary to achieve better performance. This has practical significance because having a calibration emitter near to the source is difficult to achieve, if not impossible in practice especially for non-cooperative positioning.

We next examine the placement of calibration emitter through simulations. For each of the 250 randomly generated geometries (3-D for TOA and TDOA, 2-D for AOA), 5 calibration positions are also created randomly using uniform distribution over a cube with edge length of 1000. In each ensemble run, we first obtain the source location estimate without using a calibration emitter. A calibration position is then selected based on the minimum distance to the estimated source location, denoted as min-D; the minimum of the proposed criterion $J$ in $(7.62)$, called min-J; or the minimum of the trace of the source location CRLB by taking the inverse of $(7.47)$, denoted as min-C. A final source location estimate is produced from another MLE that jointly estimates the source and sensor positions with the chosen calibration position. 
The mse results of the source location estimates averaged over the 250 geometries are shown in Figs. 7.9-7.11 for the three strategies (min-D, min-J and min-C) to select the calibration emitter, where the noise covariance settings are according to (7.29), (7.37) and (7.44). The noise powers are $\sigma_{r}^{2}=10^{-4}, \sigma_{d}^{2}=10^{-4}$ and $\sigma_{a}^{2}=10^{-4}$. For reference purpose, the minimum and the mean values of those mse results from the 5 calibration positions are also given. By observing those figures, three noticeable conclusions can be made. First, having a calibration source provides obvious performance gain compared to without using one. Second, the min-D, min-J and min-C results are all better than the mean of the mse results from the 5 calibration positions. Third, the proposed minimum $J$ criterion (min-J) yields about $2 \mathrm{~dB}$ mse reduction for TOA and TDOA and about $4 \mathrm{~dB}$ for $\mathrm{AOA}$ compared to the criterion of minimum distance to the unknown source (min-D). Indeed, min-J yields almost identical performance as min-C and they are very close to the smallest mse results from the 5 calibration positions.

One advantage the proposed $J$ criterion has over the CRLB criterion is the computation load: it's much faster to compute the $J$ value than the corresponding CRLB of the source location estimate. Table 7.1 shows the averaged computation time of the CRLB trace relative to those of the $J$ value for the three positioning cases. They are obtained from matlab and averaged over the 250 random geometries with 1000 ensemble runs each. From the results we can observe that the time required to compute the CRLB trace value is at least 11 times longer than that required for the $J$ value. The proposed $J$ criterion is much more computationally efficient. 


\subsection{Concluding Remarks}

It is often believed that an estimator needs to use the statistics of the sensor position errors in addition to those of the measurement noise to achieve the optimum CRLB performance in locating a source. This paper shows that condition exists on the covariance matrices of sensor position and measurement noise where taking the sensor position errors into account is not necessary to achieve the CRLB performance and a simpler estimator can be used instead to locate the unknown source when the noise is not excessive. The relations for TOA, TDOA and AOA are derived and they are in general dependent on the localization geometry. However, geometry independent conditions exist that can be satisfied in practice depending on applications. The optimum placement of the calibration emitter is derived for the purpose to correct the sensor positions. The optimum calibration position is at the unknown source location when the sensor position errors are IID, very large or very small, regardless the localization geometry or the noise covariance matrices. Placing a calibration emitter near the unknown source may not be a good strategy and a suboptimum criterion to allocate the calibration emitter is proposed. The suboptimum criterion yields a better calibration position than the closest to the unknown source criterion. 


\subsection{Appendix}

\subsubsection{A: Evaluation of $\operatorname{FIM}\left(\mathbf{u}^{o}\right)$ in the presence of a Calibra- tion Emitter}

The matrix $\overline{\mathbf{G}}_{s}^{T}$ defined in (7.25b) is $N M \times M$ and (7.26) indicates that it has a rank of $M$ with orthonormal columns. Let the $N M \times(N-1) M$ matrix $\overline{\mathbf{G}}_{s}^{\perp T}$ be the orthogonal complement of $\overline{\mathbf{G}}_{s}^{T}$ such that the composite matrix $\left[\overline{\mathbf{G}}_{s}^{T}, \overline{\mathbf{G}}_{s}^{\perp T}\right]$ is orthonormal and its columns span the entire space of dimension $N M$.

For $N=2$ and $i=1,2, \cdots, M$, it is easy to verify that

$$
\overline{\mathbf{G}}_{s}^{\perp}(i,:)=\left[\mathbf{0}_{N(i-1) \times 1}^{T}, q_{i}(2),-q_{i}(1), \mathbf{0}_{N(M-i) \times 1}^{T}\right],
$$

For $N=3$ and $i=1,2, \cdots, M$,

$$
\begin{aligned}
& \overline{\mathbf{G}}_{s}^{\perp}(2 i-1,:)=\frac{\left[\mathbf{0}_{N(i-1) \times 1}^{T}, \mathbf{v}_{1}^{T}, \mathbf{0}_{N(M-i) \times 1}^{T}\right]}{\left\|\mathbf{v}_{1}\right\|} \\
& \overline{\mathbf{G}}_{s}^{\perp}(2 i,:)=\frac{\left[\mathbf{0}_{N(i-1) \times 1}^{T}, \mathbf{v}_{2}^{T}, \mathbf{0}_{N(M-i) \times 1}^{T}\right]}{\left\|\mathbf{v}_{1}\right\|}
\end{aligned}
$$

where $\mathbf{v}_{1}=\left[q_{i}(2),-q_{i}(1), 0\right]^{T}, \mathbf{v}_{2}=\left[q_{i}(1) q_{i}(3), q_{i}(2) q_{i}(3),-q_{i}(1)^{2}-q_{i}(2)^{2}\right]^{T}$ and $q_{i}(j)$ is the $j$-th element of $\mathbf{q}_{i}$ defined in (7.24).

We can always decompose $\gamma_{i}$ as

$$
\gamma_{i}=a_{i} \mathbf{q}_{i}+\mathbf{V}_{i}^{\perp} \mathbf{b}_{i}
$$

where $\mathbf{V}_{i}^{\perp}$ is the orthogonal complement of $\mathbf{q}_{i}$ with orthonormal columns. For $N=2$, 
$\mathbf{b}_{i}$ is a scalar and it is a $2 \times 1$ vector when $N=3$. Since $\boldsymbol{\gamma}_{i}$ has unity norm,

$$
a_{i}^{2}+\mathbf{b}_{i}^{T} \mathbf{b}_{i}=1, \quad i=1,2, \cdots, M
$$

In other words,

$$
0<\left|a_{i}\right|<1 \text {. }
$$

Hence from (7.65),

$$
\overline{\mathbf{G}}_{c}^{T}=\overline{\mathbf{G}}_{s}^{T} \mathbf{A}+\overline{\mathbf{G}}_{s}^{\perp T} \mathbf{B}=\left[\begin{array}{ll}
\overline{\mathbf{G}}_{s}^{T} & \overline{\mathbf{G}}_{s}^{\perp T}
\end{array}\right]\left[\begin{array}{l}
\mathbf{A} \\
\mathbf{B}
\end{array}\right]
$$

where

$$
\mathbf{A}=\operatorname{diag}\left\{a_{1}, a_{2}, \cdots, a_{M}\right\}, \mathbf{B}=\left[\begin{array}{cccc}
\mathbf{b}_{1} & \mathbf{0} & \cdots & \mathbf{0} \\
\mathbf{0} & \mathbf{b}_{2} & \cdots & \mathbf{0} \\
\vdots & \vdots & \ddots & \vdots \\
\mathbf{0} & \mathbf{0} & \cdots & \mathbf{b}_{M}
\end{array}\right]
$$

Let

$$
\boldsymbol{\Sigma}_{s}=\left[\begin{array}{ll}
\boldsymbol{\Sigma}_{s, 11} & \boldsymbol{\Sigma}_{s, 12} \\
\boldsymbol{\Sigma}_{s, 12}^{T} & \boldsymbol{\Sigma}_{s, 22}
\end{array}\right]=\left[\begin{array}{l}
\mathbf{G}_{s} \\
\mathbf{G}_{s}^{\perp}
\end{array}\right] \mathbf{Q}_{s}^{-1}\left[\begin{array}{ll}
\mathbf{G}_{s}^{T} & \mathbf{G}_{s}^{\perp T}
\end{array}\right]
$$

so that

$$
\mathbf{Q}_{s}^{-1}=\left[\begin{array}{ll}
\mathbf{G}_{s}^{T} & \mathbf{G}_{s}^{\perp T}
\end{array}\right] \boldsymbol{\Sigma}_{s}\left[\begin{array}{c}
\mathbf{G}_{s} \\
\mathbf{G}_{s}^{\perp}
\end{array}\right] .
$$


Using the representations (7.68) and (7.71), $\mathbf{Z}_{c}$ in (7.48) can be expressed as

$$
\mathbf{Z}_{c}=\left[\begin{array}{ll}
\overline{\mathbf{G}}_{s}^{T} & \overline{\mathbf{G}}_{s}^{\perp T}
\end{array}\right]\left[\begin{array}{ll}
\mathbf{P}_{11} & \mathbf{P}_{12} \\
\mathbf{P}_{12}^{T} & \mathbf{P}_{22}
\end{array}\right]\left[\begin{array}{c}
\overline{\mathbf{G}}_{s} \\
\overline{\mathbf{G}}_{s}^{\perp}
\end{array}\right]
$$

where

$$
\begin{gathered}
\mathbf{P}_{11}=\boldsymbol{\Sigma}_{s, 11}+\mathbf{Q}_{m}^{-1}+\mathbf{A Q}_{c}^{-1} \mathbf{A}^{T} \\
\mathbf{P}_{12}=\boldsymbol{\Sigma}_{s, 12}+\mathbf{A Q}_{c}^{-1} \mathbf{B}^{T} \\
\mathbf{P}_{22}=\boldsymbol{\Sigma}_{s, 22}+\mathbf{B Q}_{c}^{-1} \mathbf{B}^{T}
\end{gathered}
$$

Recall that $\left[\overline{\mathbf{G}}_{s}^{T}, \overline{\mathbf{G}}_{s}^{\perp T}\right]$ is orthonormal, we have

$$
\mathbf{Z}_{c}^{-1}=\left[\begin{array}{ll}
\overline{\mathbf{G}}_{s}^{T} & \overline{\mathbf{G}}_{s}^{\perp T}
\end{array}\right]\left[\begin{array}{ll}
\mathbf{P}_{11} & \mathbf{P}_{12} \\
\mathbf{P}_{12}^{T} & \mathbf{P}_{22}
\end{array}\right]^{-1}\left[\begin{array}{c}
\overline{\mathbf{G}}_{s} \\
\overline{\mathbf{G}}_{s}^{\perp}
\end{array}\right]
$$

Using (7.26) and noting that $\overline{\mathbf{G}}_{s} \overline{\mathbf{G}}_{s}^{\perp T}=\mathbf{O}$, we have after using the definition of $\mathbf{Y}$ in $(7.4)$,

$$
\mathbf{Y} \mathbf{Z}_{c}^{-1} \mathbf{Y}^{T}=\overline{\mathbf{G}}_{u}^{T} \mathbf{Q}_{m}^{-1}\left(\mathbf{P}_{11}-\mathbf{P}_{12} \mathbf{P}_{22}^{-1} \mathbf{P}_{12}^{T}\right)^{-1} \mathbf{Q}_{m}^{-1} \overline{\mathbf{G}}_{u}
$$

where $\left(\mathbf{P}_{11}-\mathbf{P}_{12} \mathbf{P}_{22}^{-1} \mathbf{P}_{12}^{T}\right)^{-1}$ is the upper left block of $\left[\begin{array}{ll}\mathbf{P}_{11} & \mathbf{P}_{12} \\ \mathbf{P}_{12}^{T} & \mathbf{P}_{22}\end{array}\right]^{-1}$. As a result, (7.47) becomes

$$
\operatorname{FIM}\left(\mathbf{u}^{o}\right)=\overline{\mathbf{G}}_{u}^{T} \mathbf{Q}_{m}^{-1} \overline{\mathbf{G}}_{u}-\overline{\mathbf{G}}_{u}^{T} \mathbf{Q}_{m}^{-1}\left(\mathbf{P}_{11}-\mathbf{P}_{12} \mathbf{P}_{22}^{-1} \mathbf{P}_{12}^{T}\right)^{-1} \mathbf{Q}_{m}^{-1} \overline{\mathbf{G}}_{u}
$$

where the definition of $\mathbf{X}$ in (7.4) has been used. The second term in (7.76) is the 
loss of Fisher information caused by the sensor position errors.

We can reduce (7.76) to a simpler form. After substituting (7.73a),

$$
\mathbf{P}_{11}-\mathbf{P}_{12} \mathbf{P}_{22}^{-1} \mathbf{P}_{12}^{T}=\mathbf{Q}_{m}^{-1}+\tilde{\mathbf{Q}}_{c}^{-1}
$$

where

$$
\tilde{\mathbf{Q}}_{c}=\left(\boldsymbol{\Sigma}_{s, 11}+\mathbf{A Q}_{c}^{-1} \mathbf{A}^{T}-\mathbf{P}_{12} \mathbf{P}_{22}^{-1} \mathbf{P}_{12}^{T}\right)^{-1} .
$$

Note that $\tilde{\mathbf{Q}}_{c}$ is $\mathrm{PD}$. This is because $\mathbf{Z}_{c}$ in (7.48) is PD. According to the PD matrix properties [69], $\mathbf{Z}_{c}^{-1}$ is also PD and hence from (7.74) and (7.77), (7.78) is PD. We have the equality from matrix inversion lemma,

$$
\mathbf{Q}_{m}^{-1}\left(\mathbf{Q}_{m}^{-1}+\tilde{\mathbf{Q}}_{c}^{-1}\right)^{-1} \mathbf{Q}_{m}^{-1}=\mathbf{Q}_{m}^{-1}-\left(\mathbf{Q}_{m}+\tilde{\mathbf{Q}}_{c}\right)^{-1}
$$

As a result, (7.76) can be simplified to

$$
\operatorname{FIM}\left(\mathbf{u}^{o}\right)=\overline{\mathbf{G}}_{u}^{T}\left(\mathbf{Q}_{m}+\tilde{\mathbf{Q}}_{c}\right)^{-1} \overline{\mathbf{G}}_{u} .
$$

If $\tilde{\mathbf{Q}}_{c}=\mathbf{O}, \operatorname{FIM}\left(\mathbf{u}^{o}\right)$ reduces back to the FIM when the sensor position errors are absent, which is equal to $\mathbf{X}$ as defined in (7.4). Let $\tilde{\mathbf{Q}}_{c}^{o}$ be $\tilde{\mathbf{Q}}_{c}$ when $\mathbf{c}=\mathbf{c}^{o}$. We define the optimum calibration position as $\mathbf{c}^{o}$ such that $\tilde{\mathbf{Q}}_{c}>\tilde{\mathbf{Q}}_{c}^{o}$ for all $\mathbf{c} \neq \mathbf{c}^{o}$, where $\tilde{\mathbf{Q}}_{c}>\tilde{\mathbf{Q}}_{c}^{o}$ means that $\tilde{\mathbf{Q}}_{c}-\tilde{\mathbf{Q}}_{c}^{o}$ is $\mathrm{PD}$.

The dependency of $\tilde{\mathbf{Q}}_{c}$ on $\mathbf{c}$ is through the matrices $\mathbf{A}$ and $\mathbf{B}$ defined in (7.69) using the vector decomposition in (7.65). Hence we will determine the $\mathbf{A}$ and $\mathbf{B}$ corresponding to $\mathbf{c}^{o}$ for achieving $\tilde{\mathbf{Q}}_{c}>\tilde{\mathbf{Q}}_{c}^{o}$, subject to the $M$ constraints in (7.66) for their elements. 


\subsubsection{B: Maximizing (7.80) in the PD Sense under Special}

\section{Cases}

$\mathbf{Q}_{s}=\sigma_{s}^{2} \mathbf{I}$

When $\mathbf{Q}_{s}$ is proportional to an identity matrix, i.e. the sensor position errors are IID in each coordinate, we have

$$
\begin{aligned}
& \boldsymbol{\Sigma}_{s, 11}=\frac{1}{\sigma_{s}^{2}} \mathbf{I}_{M}, \boldsymbol{\Sigma}_{s, 12}=\mathbf{O}_{M \times(N-1) M}, \\
& \boldsymbol{\Sigma}_{s, 22}=\frac{1}{\sigma_{s}^{2}} \mathbf{I}_{(N-1) M} .
\end{aligned}
$$

Putting them to (7.73) and noting that $\mathbf{P}_{12} \mathbf{P}_{22}^{-1} \mathbf{P}_{12}^{T}$ is positive semi-definite, we obtain $\tilde{\mathbf{Q}}_{c}^{o}$ when $\mathbf{B}=\mathbf{O}$. In such a case, $\mathbf{P}_{12} \mathbf{P}_{22}^{-1} \mathbf{P}_{12}^{T}$ is zero and $\tilde{\mathbf{Q}}_{c}^{o}$ is

$$
\tilde{\mathbf{Q}}_{c}^{o}=\left(\frac{1}{\sigma_{s}^{2}} \mathbf{I}+\mathbf{Q}_{c}^{-1}\right)^{-1}
$$

Using (7.82) in (7.80) gives the maximum achievable FIM in the PD sense.

When $\mathbf{B}=\mathbf{O}$, according to (7.65) the optimum calibration position is the same as the unknown source location:

$$
\mathbf{c}^{o}=\mathbf{u}^{o}
$$

$\mathrm{Q}_{s} \ll \mathbf{Q}_{c}$

If $\mathbf{Q}_{s}$ is small relative to $\mathbf{Q}_{c}$, we have from (7.73) the approximations

$$
\mathbf{P}_{12} \simeq \boldsymbol{\Sigma}_{s, 12}, \quad \mathbf{P}_{22} \simeq \boldsymbol{\Sigma}_{s, 22}
$$


Hence $\mathbf{P}_{12} \mathbf{P}_{22}^{-1} \mathbf{P}_{12}^{T}$ is nearly independent of the calibration emitter position. $\tilde{\mathbf{Q}}_{c}^{o}$ appears when $\mathbf{A}=\mathbf{I}$, which implies the optimum $\mathbf{c}$ is the same as (7.83).

An interesting point to note is that if $\mathbf{Q}_{s} \rightarrow \mathbf{O}, \boldsymbol{\Sigma}_{s, 11}$ is very large and $\tilde{\mathbf{Q}}_{c} \simeq$ $\left(\boldsymbol{\Sigma}_{s, 11}\right)^{-1} \simeq$ O. According to $(7.80), \operatorname{FIM}\left(\mathbf{u}^{o}\right)$ reduces to the one without sensor position errors as expected.

$\mathbf{Q}_{s} \gg \mathbf{Q}_{c}$

If $\mathbf{Q}_{s}$ is big relative to $\mathbf{Q}_{c}$, we have

$$
\mathbf{P}_{12} \simeq \mathbf{A Q}_{c}^{-1} \mathbf{B}^{T}, \quad \mathbf{P}_{22} \simeq \mathbf{B Q}_{c}^{-1} \mathbf{B}^{T}+\Sigma_{s, 22}
$$

The solution is, again, $\mathbf{A}=\mathbf{I}$ or $\mathbf{B}=\mathbf{O}$ so that $\mathbf{P}_{12} \mathbf{P}_{22}^{-1} \mathbf{P}_{12}^{T}=\mathbf{O}$. $\tilde{\mathbf{Q}}_{c}^{o}$ in this case is, from (7.78),

$$
\tilde{\mathbf{Q}}_{c}^{o} \simeq \mathbf{Q}_{c}
$$

where $\boldsymbol{\Sigma}_{s, 11}$ is small enough to be neglected. The largest FIM in the PD sense is simply

$$
\operatorname{FIM}\left(\mathbf{u}^{o}\right)=\overline{\mathbf{G}}_{u}^{T}\left(\mathbf{Q}_{m}+\mathbf{Q}_{c}\right)^{-1} \overline{\mathbf{G}}_{u} .
$$

Based on the solution $\mathbf{A}=\mathbf{I}$, the optimum calibration sensor position is the same as given by (7.83). 
Table 7.1: Averaged computation time of the proposed $J$ criterion and the corresponding CRLB criterion of the source location estimate. The results are averaged over the 250 random geometries when the sensor position noise power is $10^{0.5}$ for TOA and AOA, 0.01 for TDOA.

\begin{tabular}{|c|c|c|}
\hline & Proposed $J$ criterion & CRLB criterion \\
\hline TOA & 1 & 25.78 \\
\hline TDOA & 1 & 21.21 \\
\hline AOA & 1 & 11.89 \\
\hline
\end{tabular}

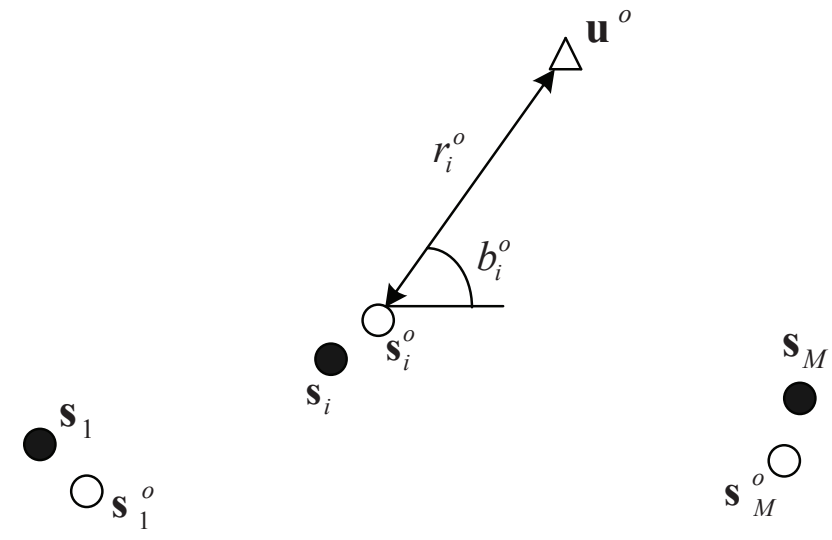

Figure 7.1: Localization scenario. Open circles denote the true sensor positions that are not known and closed circles are the available sensor positions that are erroneous. 


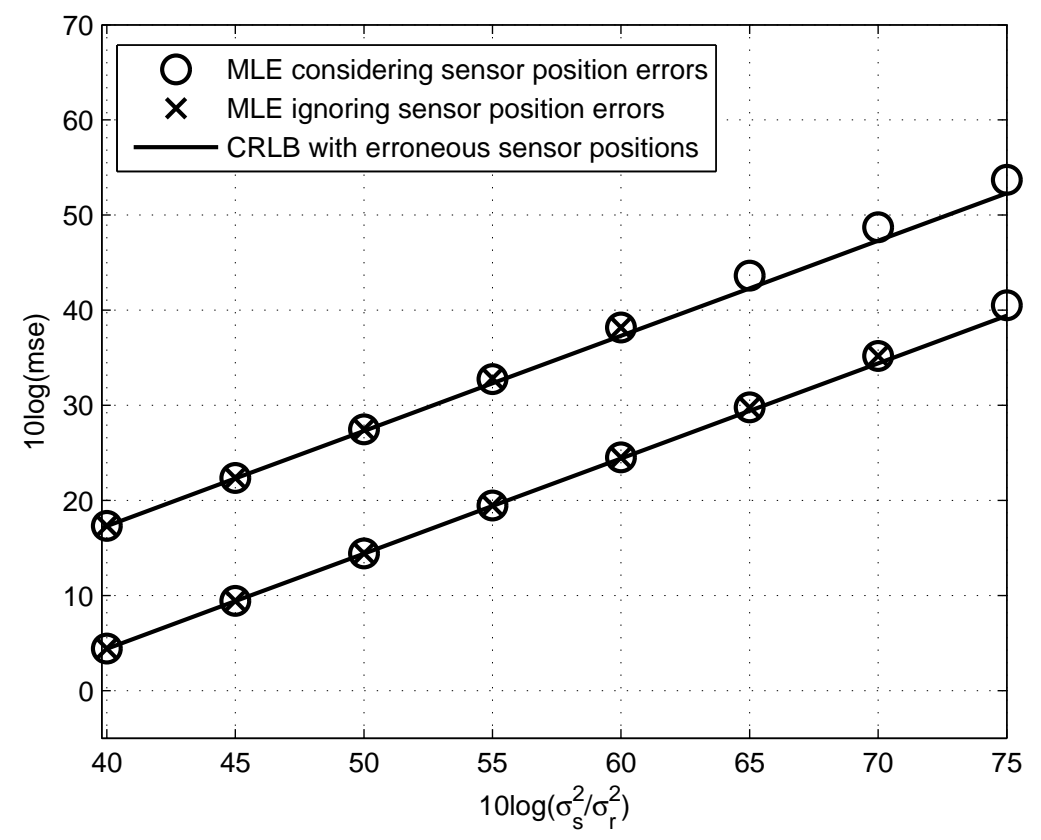

Figure 7.2: Source location estimate mse results of MLEs considering and ignoring sensor position errors using TOA measurements as $\sigma_{s}^{2}$ varies.

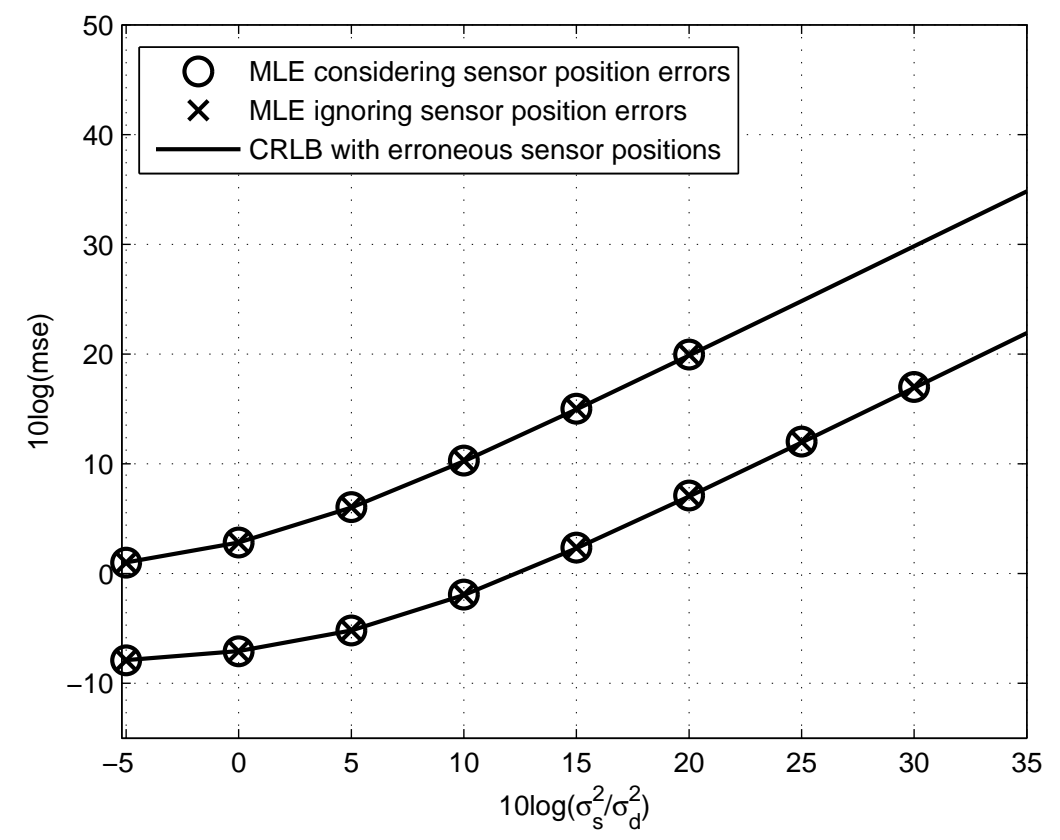

Figure 7.3: Source location estimate mse results of MLEs considering and ignoring sensor position errors using TDOA measurements as $\sigma_{s}^{2}$ varies. 


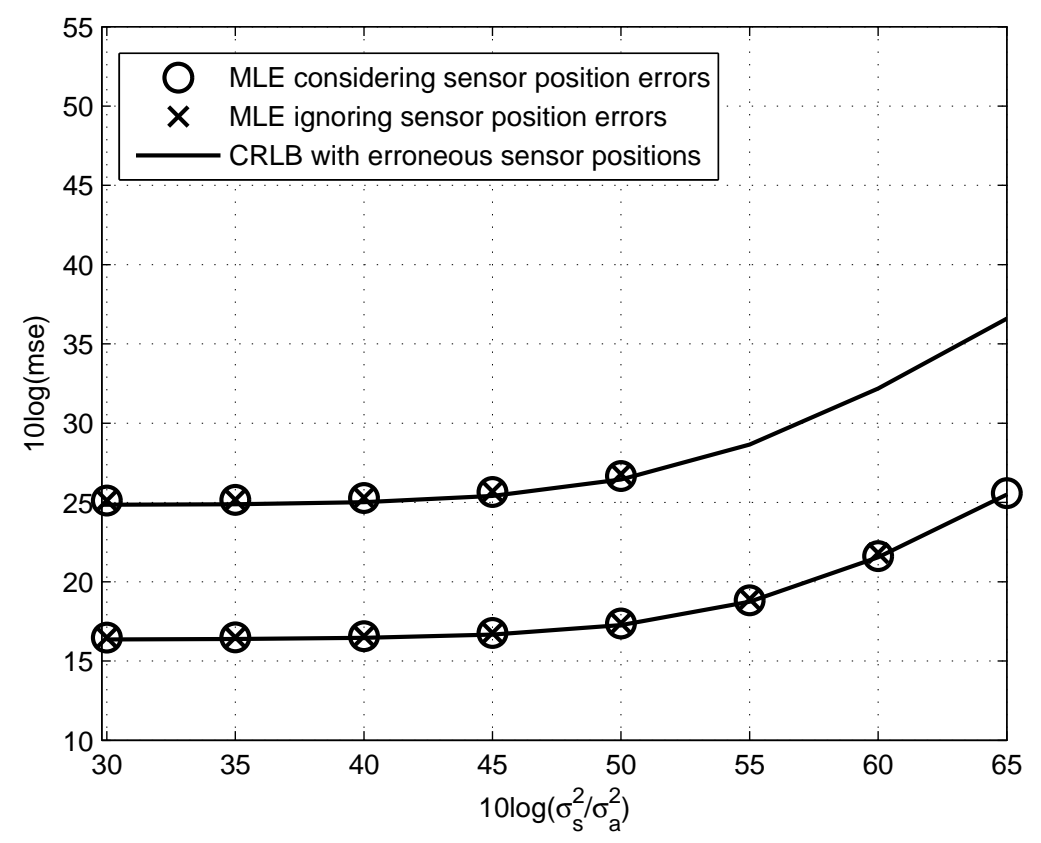

Figure 7.4: Source location estimate mse results of MLEs considering and ignoring sensor position errors using AOA measurements as $\sigma_{s}^{2}$ varies.

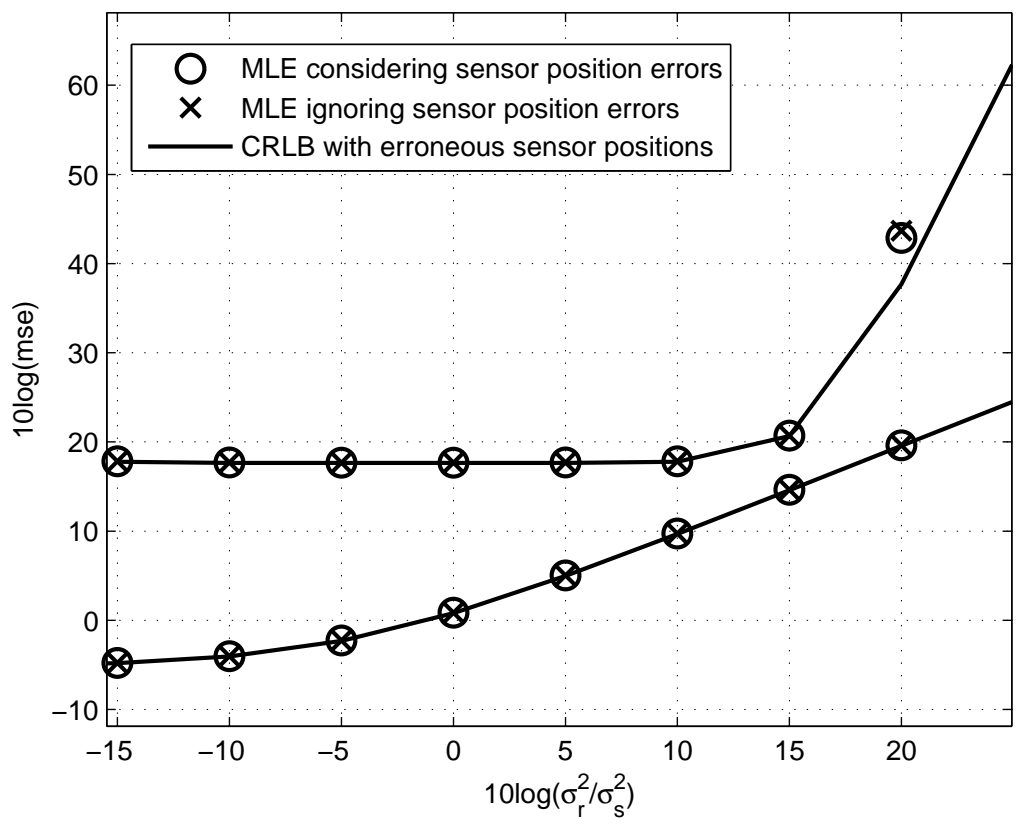

Figure 7.5: Source location estimate mse results of MLEs considering and ignoring sensor position errors using TOA measurements as $\sigma_{r}^{2}$ varies. 


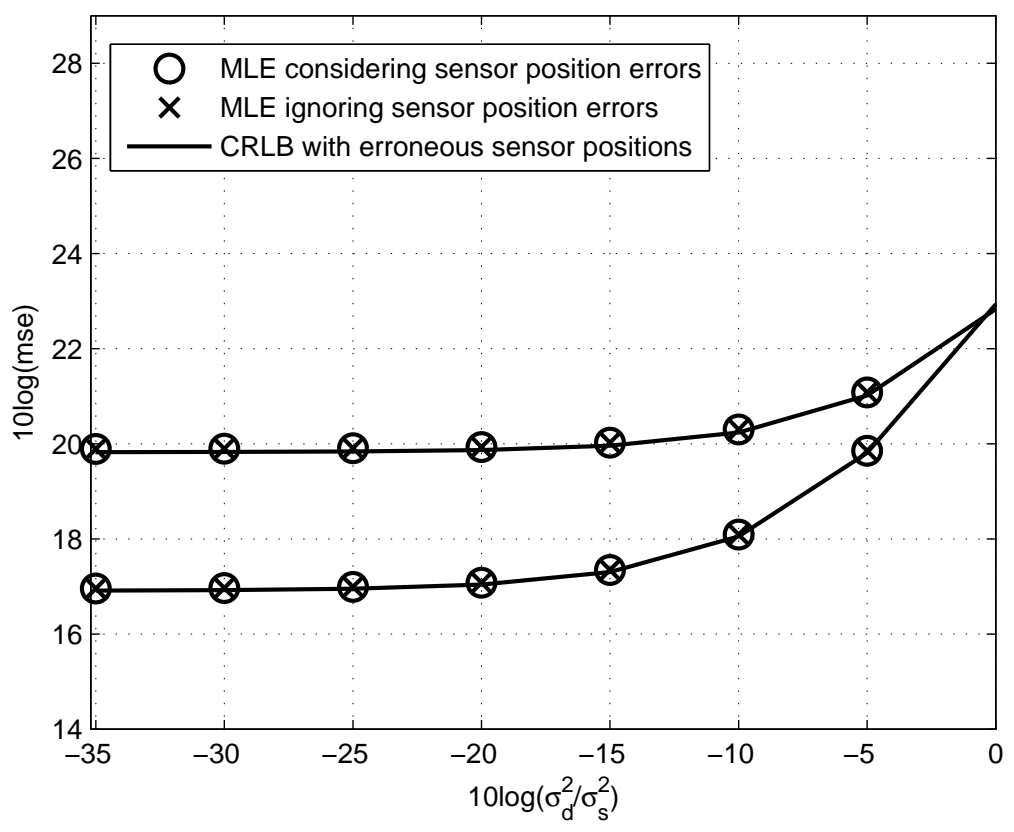

Figure 7.6: Source location estimate mse results of MLEs considering and ignoring sensor position errors using TDOA measurements as $\sigma_{d}^{2}$ varies.

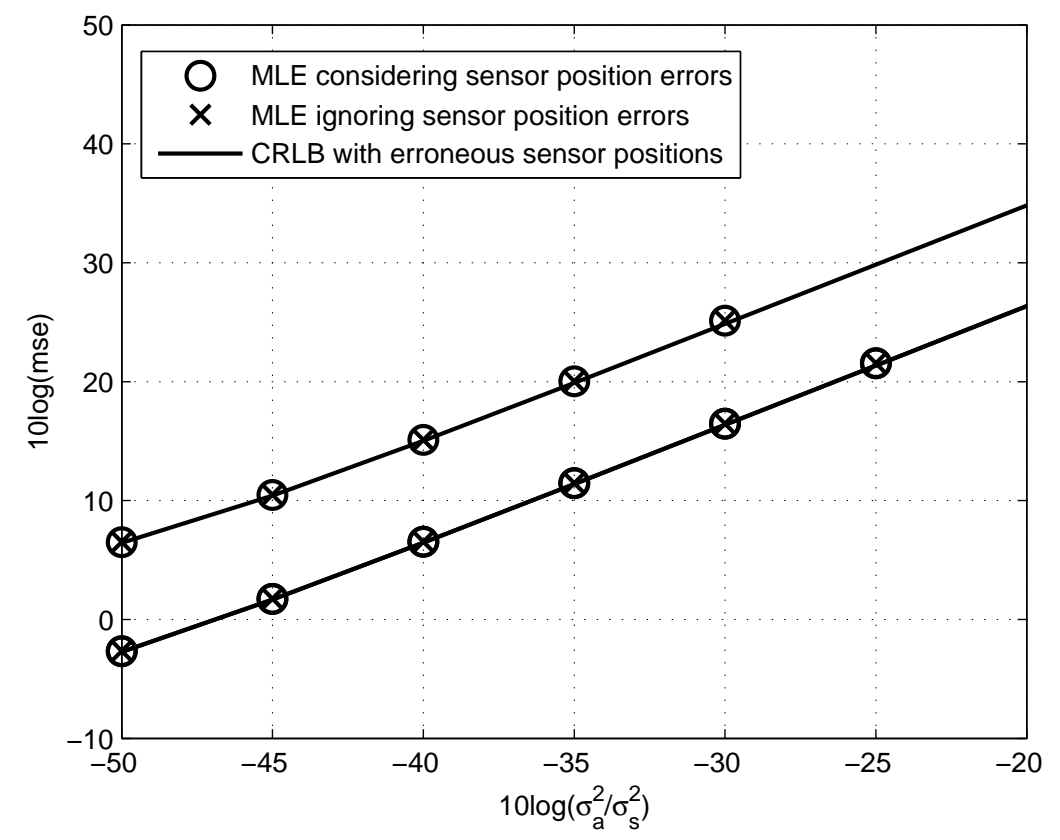

Figure 7.7: Source location estimate mse results of MLEs considering and ignoring sensor position errors using AOA measurements as $\sigma_{a}^{2}$ varies. 


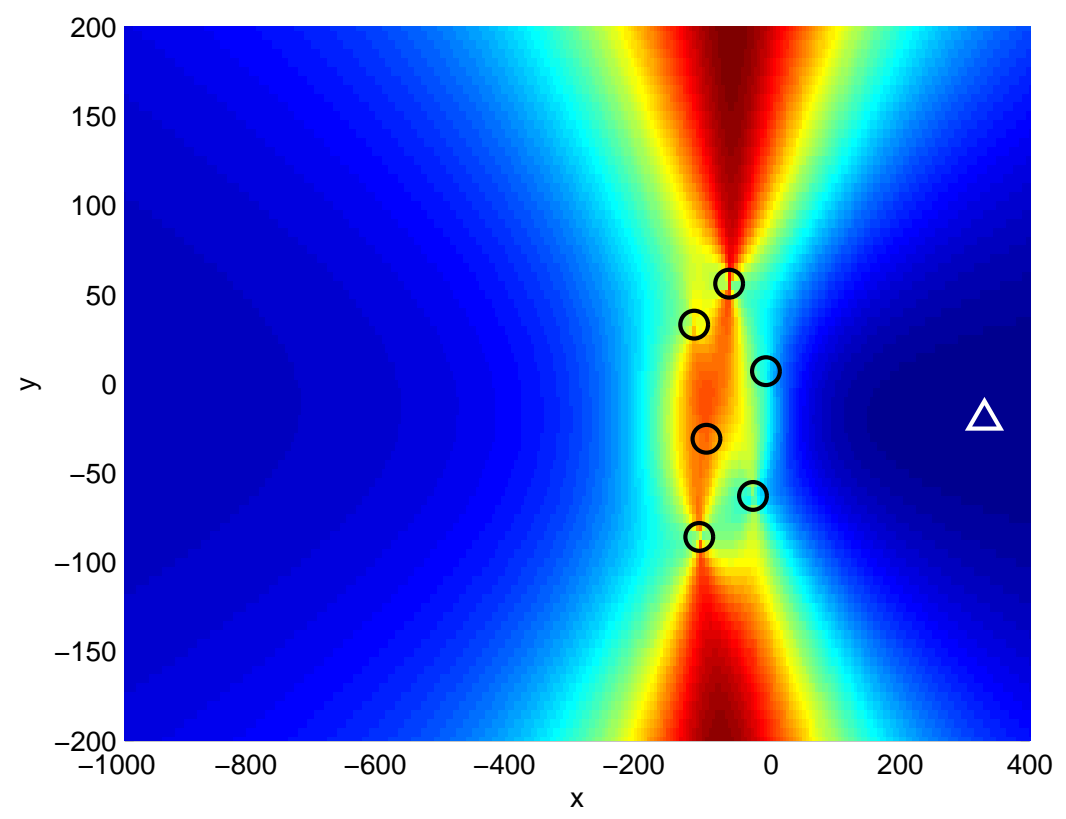

Figure 7.8: Theoretical source location estimate mse for 2-D localization with a calibration source position defined by the axes using TOA measurements, darker (bluer) level represents smaller mse.

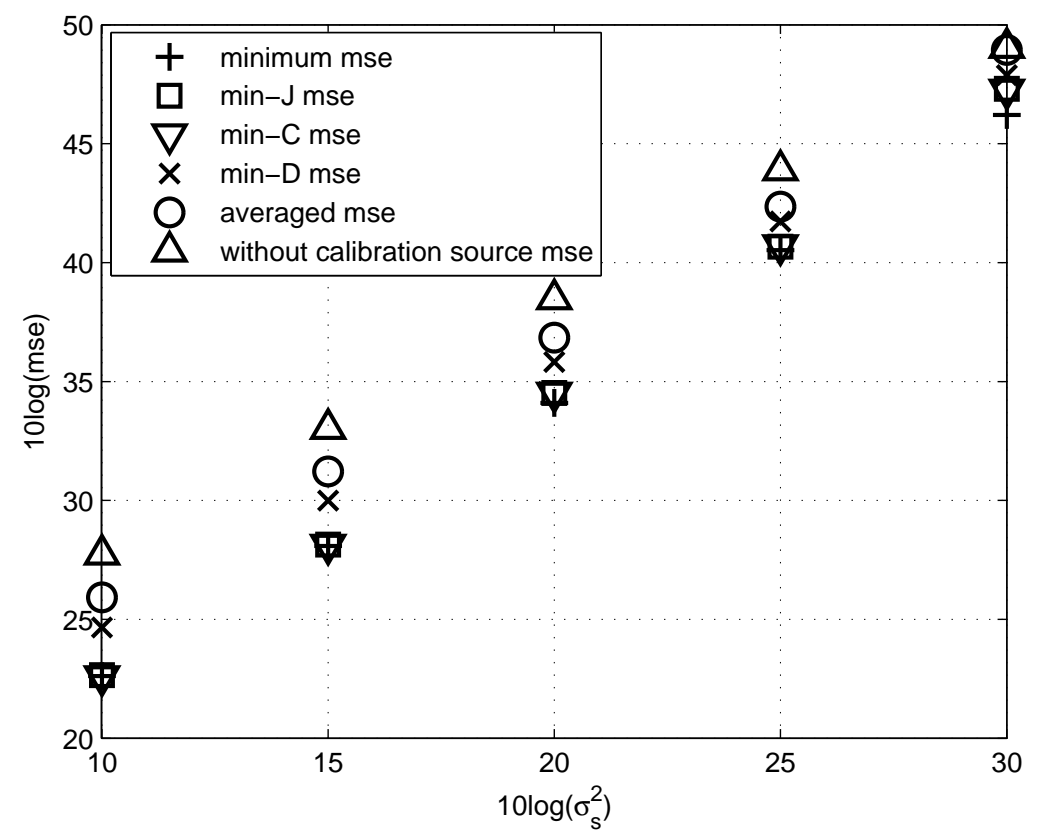

Figure 7.9: Source location estimate mse results from the 5 calibration sources using TOA measurements as $\sigma_{s}^{2}$ varies. 


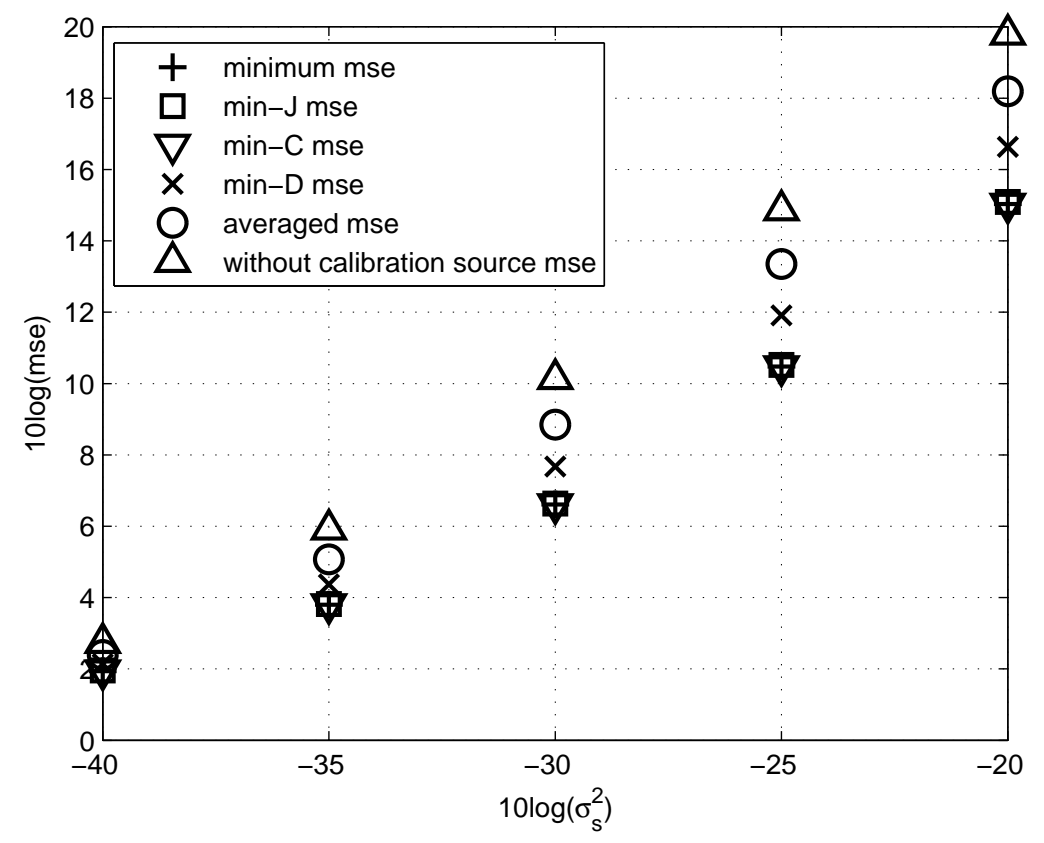

Figure 7.10: Source location estimate mse results from the 5 calibration sources using TDOA measurements as $\sigma_{s}^{2}$ varies.

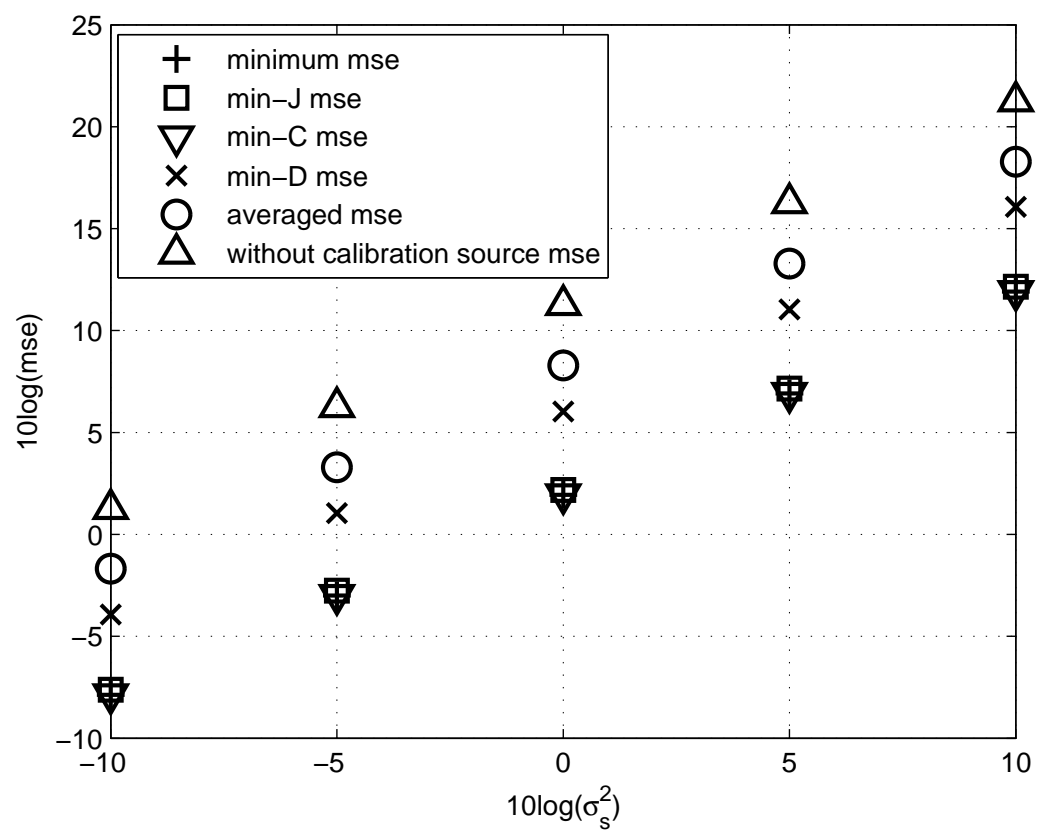

Figure 7.11: Source location estimate mse results from the 5 calibration sources using AOA measurements as $\sigma_{s}^{2}$ varies. 


\section{Chapter 8}

\section{Summary and Future Work}

In this chapter we summarize the research works that have been presented in this thesis. We shall also discuss some possible research topics we intend to conduct in the future.

\subsection{Research Summary}

The nonlinear estimation problem has been known as a challenging yet very important problem in the field of digital signal processing. The implicit relation between the measurement data and the unknown parameters to be estimated makes the nonlinear estimation problem hard to solve. Based on the Taylor-series expansion, a ML estimator can be developed to solve the nonlinear estimation problem via iterations. However, the estimate accuracy can be highly dependent on how the estimator is initialized. Poor initialization would lead to local minima instead of global minima which we are really interested in. The research presented in this thesis aim to implement digital signal processing techniques to the nonlinear estimation problem in order 
to provide a computational effective, asymtotically efficient and closed-form solution.

We consider two kinds of nonlinear estimation problems here: the circle and ellipse fittings, and the source localization. Both of them are classic nonlinear estimation problems and continue to attract research interests in recent years.

For the circle and ellipse fittings, we first examined two widely used circle estimation methods in Chapter 2: the ML estimator and the FLS estimator. Based on their cost functions, we derived iterative solutions for both methods using the Taylorseries expansion. After the two solutions are obtained, we further compared their cost functions and analytically showed that the FLS solution approximates the ML solution if the noise power is much less than the circle radius square. Otherwise, the ML solution will have better performance than the FLS one.

The ML estimator we developed in Chapter 2 has an iterative solution based on the Taylor-series expansion. However, it might suffer from the initialization problem where if the initial solution guess is not close enough to the true one, the final solution will converge to a local minimum instead of a global one. In order to handle the initialization issue, in Chapter 3 we proposed a new implementation of the ML estimator using the SDR and SDP techniques. The SDR and SDP techniques are well-known techniques for solving the optimization problem. The major advantage of using the SDP technique is that an optimum global convergence solution can be guaranteed. In order to solve the nonlinear circle fitting problem using the SDP method, we first reformulated the ML cost function and its constraints. Then we applied the SDR technique to relax the matrix rank constraint and translated the minimization of the ML cost function from a nonconvex problem to an approximate but convex one. Eventually, a SDP solver was used to estimate the circle parameters. 
The two ML solutions we derived in Chapter 2 and 3 are both for the fitting of a single circle. However, the fitting of coupled objects, such as concentric circles and concentric ellipses, is also a very common and important problem in practice. We proposed two estimators in Chapter 4 for the fittings of concentric circles and concentric ellipses. The asymptotically efficient estimators we developed are based on two digital signal processing techniques: the weighted equation error formulation and the nonlinear parameter transformation. As a result, they can provide explicit solutions, do not require iterations and do not suffer from the initialization problem. We also developed the KCR bounds for the concentric circle and concentric ellipse parameters under Gaussian noise as a benchmark to evaluate the performances of the proposed estimators. In the end of the chapter, we applied the the concentric ellipse estimator to a main application of ellipse fitting in practice: iris recognition. Real eye images were used for the fitting and the results verified the good performance of the proposed estimator.

The iterative solution from Chapter 2 requires a good initial solution guess while the SDP one in Chapter 3 can guarantee an optimum global convergence solution but with a dramatic increase in computation. The proposed concentric circle and concentric ellipse fitting methods in Chapter 4 can be reduced back to the fittings of a single circle and a single ellipse in straightforward manners. For the fitting of a single circle, the proposed estimator can provide a closed-form solution to avoid the iterations and good initial solution guess requirement as in the ML estimator. Also it has much less computation load comparing with the SDP solution. More importantly, the proposed estimators can reach the KCR bounds accuracy even for the anisotropic noise. 
The localization of a source using measurements from a collection of sensors is another important nonlinear estimation problem we considered in this thesis. Many studies have been conducted to show that the accuracy of a source location estimate could be degraded significantly due to the sensor position uncertainties when the TDOA measurements are used. Our work in Chapter 5 dealt with the source localization problem based on the TOA measurements. We first derived the CRLB of a source location when sensor position errors are present and compared it with the MSE of a source location when ignoring the sensor position errors. Via the comparison, the estimation performance loss due to the sensor position uncertainties was shown analytically. A closed-form solution that accounts for the sensor position errors was then proposed. In such a way, we were able to not only theoretically analyze the degradation in accuracy of the source location estimate, but also provide an explicit solution in the presence of sensor position errors. The proposed efficient estimator was shown via simulations to reach the CRLB performance when the noise level is small.

One can improve the sensor positions by using one or multiple sources, often called calibration sources or anchors, that are at known locations. However, deploying a calibration source could be costly. In Chapter 6 we have developed an algebraic solution that jointly estimates the positions of multiple sources and sensors. The proposed method is able to achieve the CRLB performance for both the source and the sensor locations. The refined sensor positions can improve the localization of newly appeared sources subsequently. The good performance of the proposed estimator is shown analytically and supported by simulations. Compared to the sequential estimation-refinement technique, the proposed estimator provides better performance 
in sensor position estimates at higher noise level.

The CRLB and MSE studies presented in Chapter 5 are for the TOA measurement only. In Chapter 7 we extended the CRLB and MSE studies for the TDOA and AOA cases and presented the analysis in a general form for the three measurements. Through the analysis and the comparison results, we were able to show that in the presence of sensor position errors, there are situations exist where taking into account the sensor position errors when estimating the source location will not improve the estimation accuracy. Under these situations, the CRLB when the sensor position errors are present is equal to the MSE where the sensor position errors are ignored but in fact exist. We also provided more details on what these situations would be for the TOA, TDOA and AOA measurements respectively. We have also shown that in the presence of sensor position errors, a calibration source with exactly known position can be used to improve the localization performance further. The amount of improvement of the source location estimate accuracy because of the calibration source would be highly depended on the calibration source position. We investigated where would be the optimum position to place the calibration source so that the source location estimate accuracy would be improved maximally in the presence of sensor position uncertainties. We also showed that placing a calibration emitter near the unknown source may not be a good strategy and a suboptimum criterion to allocate the calibration emitter is proposed. The suboptimum criterion yields a better calibration position than the closest to the unknown source criterion. 


\subsection{Future Research Work}

In Chapter 3 we applied the SDR and SDP techniques on the fitting of a single circle. The resulted estimator can guarantee an optimum global convergence solution, which is a major advantage over other iterative circle fitting methods that might suffer from the initialization problem. One possibility of our future research is that we can implement the SDR and SDP techniques to the fitting of a single ellipse, which is more difficult to deal with comparing to the circle fitting problem. To the best of our knowledge, not much work has been done regarding to this aspect. After that, we can also extend the SDP based single circle/ellipse fitting method to the fitting of concentric circles/ellipses. Our hope is that the resulted concentric circle/ellipse estimator can provide better noise resistance comparing to the one we presented in Chapter 4 .

The CRLB and MSE studies as well as the optimum calibration placement investigation we accomplished in Chapter 7 are all regarding to the stationary source and sensors. When there are relative motions between the sensors and the source, we known from [13] that the FDOA measurements can be combined with the TDOA measurements to jointly locate the source. As a result, we can consider extending the CRLB and MSE studies further to include this TDOA and FDOA source localization problem where there are errors in both the sensor positions and velocities. Our objective will still be seeking whether situation exists that even taking into account the uncertainties in the sensor positions and velocities when estimating the source location would not help to improve the estimation performance. Also, the optimum calibration placement investigation can be conducted for the dynamic source and sensors case. In this case both the calibration TDOA and calibration FDOA measurements 
will be used to determine the optimum position of the calibration source. 


\section{Bibliography}

[1] S.D. Connell and A.K. Jain, "Template-based online character recognition," Pattern Recognit., vol. 34, pp. 1-14, 2001.

[2] M. Shpitalni and H. Lipson, "Classification of sketch strokes and corner detection using conic sections and adaptive clustering," Trans. ASME, J. Mech. Des., vol. 119, pp. 131-135, Mar. 1997.

[3] A.E. Yahya and M.J. Nordin, "A new technique for iris localization in iris recognition systems," Information Technol. J., vol. 7, pp. 924-929, 2008.

[4] P. Núẽz, R. Vázquez-Martin, A. Bandera, and F. Sandoval, "An algorithm for fitting 2-D data on the circle: applications to mobile robotics," IEEE Signal Process. Lett., vol. 15, pp. 127-130, 2008.

[5] D.T. Lin and C.M. Yang, "Real-time eye detection using face circle fitting and dark-pixel filtering," in Proc. IEEE Int. Conf. Multimedia and Expo (ICME), Taipei, Jun. 2004, pp. 1167-1170.

[6] S. M. Kay, Fundamentals of Statistical Signal Processing: Estimation Theory. Prentice Hall, NJ, 1993. 
[7] Y.T. Chan, B.H. Lee and S.M. Thomas, "Approximate Maximum Likelihood Estimation of Circle Parameters," Journal of Optimization Theory and Applications, Vol. 125, No. 3, pp. 723-734, June 2005.

[8] Y.E. Nesterov, "Semidefinite relaxation and nonconvex quadratic optimization," Optim. Methods Softw., vol. 9, pp. 140-160, 1998.

[9] P. Benko, G. Kós, T. Várady, L. Andor, and R. Martin, "Constrained fitting in reverse engineering," Comput.-Aided Geom. Des., vol. 19, 2002.

[10] P. O'Leary, M. Harker, and P. Zsombor-Murray, "Direct and least square fitting of coupled geometric objects for metric vision," IEE Proc.-Vis. Image Signal Process., vol. 152, pp. 687-694, Dec. 2005.

[11] Y.T. Chan, H. Y. C. Hang and P. C. Ching, "Exact and approximate maximum likelihood localization algorithms," IEEE Trans. Veh. Technol., vol. 55, pp. 1016, Jan. 2006.

[12] A.H. Sayed, A. Tarighat and N. Khajehnouri, "Network-based wireless location: challenges faced in developing techniques for accurate wireless location information," IEEE Signal Processing Mag., vol. 22, pp. 24-40, Jul. 2005.

[13] K.C. Ho, X. Lu and L. Kovavisaruch, "Source localization using TDOA and FDOA measurements in the presence of receiver location errors: analysis and solution," IEEE Trans. Signal Process., vol. 55, pp. 684-696, Feb. 2007.

[14] J. Prieto, S. Mazuelas, A. Bahillo, P. Fernandez, R. M. Lorenzo, and E. J. Abril, "Adaptive data fusion for wireless localization in harsh environments," IEEE Trans. Signal Process., vol. 60, pp. 1585-1596, Apr. 2012. 
[15] K.W.K. Lui, W.-K. Ma, H.C. So, and F.K.W. Chan, "Semi-definite programming algorithms for sensor network node localization with uncertainties in anchor positions and/or propogation speed," IEEE Trans. Signal Processing, vol. 57, pp. 752-763, Feb. 2009.

[16] S. Srirangarajan, A. H. Tewfik and Z.-Q. Luo, "Distributed sensor network localization with inaccurate anchor positions and noisy distance information," in Proc. Int. Conf. Acoust. Speech, Signal Process., vol. 3, pp. 521-524, Apr. 2007.

[17] J. Zheng and Y.-C. Wu, "Robust joint localization and time synchronization in wireless sensor networks with bounded anchor uncertainties," in Proc. Int. Conf. Acoust. Speech, Signal Process., pp. 2793-2796, Apr. 2009.

[18] Y. Rockah and P. M. Schultheiss, "Array shape calibration using source in unknown location, Part I: Far-field source," IEEE Trans. Acoust., Speech, Signal Process., vol. ASSP-35, pp. 286-299, Mar. 1987.

[19] M. Crocco, A. Del Bue, M. Bustreo, and V. Murino, "A closed form solution to the microphone position self-calibration problem," in Proc. Int. Conf. Acoust. Speech, Signal Process., ICASSP-2012, Kyoto, Japan, Mar. 2012, pp. 2597 2600.

[20] M. Sun and K.C. Ho, "Refining inaccurate sensor positions using target at unknown location," Elsevier Int. J. Signal Processing, vol. 92, pp. 2097-2104, Sep. 2012.

[21] M. Sun, L. Yang, and K.C. Ho, "Efficient joint source and sensor localization in closed-form," IEEE Signal Process. Letters, vol. 19, pp. 399 - 402, Jul. 2012. 
[22] K. C. Ho and L. Yang, "On the use of a calibration emitter for source localization in the presence of sensor position uncertainty," IEEE Trans. Signal Process., vol. 56, pp. 5758 - 5772, Dec. 2008.

[23] I. Kåsa, "A circle fitting procedure and its error analysis," IEEE Trans. Instrum. Meas., vol. 25, pp. 8-14, Mar. 1976.

[24] D. Umbach and K.N. Jones, "A few methods for fitting circles to data," IEEE Trans. Instrum. Meas., vol. 52, pp. 1881-1885, Dec. 2003.

[25] V. Pratt, "Direct least-squares fitting of algebraic surfaces," Comp. Graph., vol. 21, pp. 145-152, July 1987.

[26] N. Chernov and C. Lesort, "Least squares fitting of circles," J. Math. Imag. Vis., vol. 23, pp. 239-252, Nov. 2005.

[27] E.E. Zelniker and I.V.L. Clarkson, "A statistical analysis of the Delogne-Kåsa method for fitting circles," Digit. Signal Process., vol. 16, pp. 498-522, Sept. 2006.

[28] N. Chernov and C. Lesort, "Statistical efficiency of curve fitting algorithms," Comp. Stat. Data Anal., vol. 47, pp. 713-728, Nov. 2004.

[29] E.E. Zelniker, B.C. Appleton, and I.V.L. Clarkson, "Optimal circle fitting via branch and bound," in Proc. IEEE Int. Conf. Acous., Speech, Signal Process. (ICASSP), Philadelphia, PA, USA, Mar. 2005, pp. 709-712.

[30] N. Chernov and P. N. Sapirstein, "Fitting circles to data with correlated noise," Comp. Stat. Data Anal., vol. 52, pp. 5328-5337, Aug. 2008. 
[31] G. Taubin, "Estimation of planar curves, surfaces, and nonplanar space curves defined by implicit equations with applications to edge and range image segmentation," IEEE Trans. Pattern Anal. Machine Intell., vol. 13, pp. 1115-1138, Nov. 1991.

[32] P.L. Rosin, "A note on the least squares fitting of ellipse," Pattern Recognit. Lett., vol. 14, pp. 799-808, Oct. 1993.

[33] A. Fitzgibbon, M. Pilu, and R.B. Fisher, "Direct least square fitting of ellipses," IEEE Trans. Pattern Anal. Machine Intell., vol. 21, pp. 476-480, May 1999.

[34] W. Chojnacki, M.J. Brooks, A. van den Hengel, and D. Gawley, "On the fitting of surfaces to data with covariances," IEEE Trans. Pattern Anal. Machine Intell., vol. 22, pp. 1294-1303, Nov. 2000.

[35] Y. Leedan and P. Meer, "Heteroscedastic regression in computer vision: problems with bilinear constraint," Int. J. Computer Vision, vol. 37, pp. 127-150, Jun. 2000 .

[36] B. Matei and P. Meer, "A general method for Errors-in-Variables problems in computer vision," in Proc. IEEE Int. Conf. Comput. Vision Pattern Recognit. (CVPR), Hilton Head Island, SC, USA, Jun. 2000, pp. 18-25.

[37] K. Kanatani, "Renormalization for unbiased estimation," in Proc. 4th Int. Conf. Comput. Vision, Berlin, Germany, May 1993, pp. 599-606.

[38] J.-S. Kim, H.-W. Kim, and I.-S. Kweon, "A camera calibration method using concentric circles of vision applications," in Proc. Asian Conf. Comput. Vision, Melbourne, Australia, 2002, pp. 512-520. 
[39] S.D. Dampegama, "Determination of original size of the abhayagiriya stupa," in 21st Asian Conf. on Remote Sens., Taipei, 2000.

[40] J. Marot and S. Bourennane, "Subspace-based and DIRECT algorithms for distorted circular contour estimation," IEEE Trans. Image Process., vol. 16, pp. 2369-2378 Sept. 2007.

[41] L. Lovász, "On the Shannon capacity of a graph," IEEE Trans. Inform. Theory, vol. IT-25, pp. 1-7, 1979.

[42] M.X. Goemans and D.P. Williamson, "Improved approximation algorithms for maximum cut and statisfiability problem using semi-definite programming," $J$. ACM, vol. 42, pp. 1115-1145, Nov. 1995.

[43] Z.-Q. Luo, W.-K. Ma, A.M.-C. So, Y. Ye, and S. Zhang, "Semidefinite relaxation of quadratic optimization problems," IEEE Signal Process. Mag., vol. 27, pp.2034, May 2010.

[44] K.W. Cheung, W.-K. Ma, and H.C. So, "Accurate approximation algorithm for TOA-based maximum likelihood mobile location using semidefinite programming" in Proc. IEEE Int. Conf. Acous., Speech, Signal Process. (ICASSP), Montreal, Canada, Mar. 2004, pp. 145-148.

[45] J. Sun, R. Freund and T. Magnanti, 15.094J Systems Optimization: Models and Computation (SMA 5223), Spring 2004. (Massachusetts Institute of Technology: MIT OpenCourseWare), http://ocw.mit.edu.

[46] N. Liu, Z. Xu, and B. M. Sadler, "Geolocation performance with biased range measurements," IEEE Trans. Signal Process., vol. 60, pp. 2315-2329, May 2012. 
[47] J. Shen and A. F. Molisch, "Indirect path detection based on wireless propagation measurements," IEEE Trans. Wireless Commun., vol. 11, pp. 4482-4493, Dec. 2012.

[48] Y.M. Chen, J.H. Lee, and C.C. Yeh, "Two-dimensional angle-of-arrival estimation for uniform plannar array with sensor position errors," Proc. Inst. Elect. Eng.-Radar, Signal Process., vol. 140, pp. 37-42, Feb. 1993.

[49] S.M. Thomas and Y.T. Chan, "Cramer-Rao Lower Bounds for Estimation of a Circular Arc Center and Its Radius," Graghical Models and Image Processing, Vol. 57, pp. 527-532, Nov. 1995.

[50] J. Löfberg, "YALMIP: a toolbox for modeling and optimization in MATLAB," in Proc. Int. Symp. CACSD, pp. 284 - 289, Taipei, Taiwan, Sept. 2004.

[51] R.H. Tutuncu, K.C. Toh, and M.J. Todd, "Solving semidefinite-quadratic-linear programs using SDPT3," Mathematical Programming Ser. B, vol. 95, pp. 189 $217,2003$.

[52] P. Stoica, K. Sharman, Maximum likelihood methods for direction of arrival estimation, IEEE Trans. Acoust., Speech, Signal Process., ASSP-38 (1990) 11321143.

[53] P.J. Green, Iteratively reweighted least squares for maximum likelihood estimation, and some robust and resistant alternatives, J. Roy. Statist. Soc. Series B, 46 (1984) 149-192.

[54] K. Kanatani, "Cramér-Rao lower bounds for curve fitting," Graph. Models Image Process., vol. 60, pp. 93-99, Mar. 1998. 
[55] K. Kanatani, Statistical optimization for geometric fitting: theoretical accuracy bound and higher order error analysis, Int. J. Comput. Vis. 80 (2008) 167-188.

[56] CASIA Iris Image Database, http://biometrics.idealtest.org/.

[57] J. Canny, "A computational approach to edge detection," IEEE Trans. Pattern Anal. Machine Intell., vol. 8, pp. 679-698, Nov. 1986.

[58] J.K. Pillai, V.M. Patel, R. Chellappa, and N.K. Ratha, "Secure and robust iris recognition using random projections and sparse representations," IEEE Trans. Pattern Anal. Machine Intell., vol. 33, pp. 1877-1893, Sept. 2011.

[59] A.W.-K. Kong, "IrisCode decompression based on the dependence between its bit pairs," IEEE Trans. Pattern Anal. Machine Intell., vol. 34, pp. 506-520, Mar. 2012.

[60] M. Harker and P. O'Leary, Geometric Library for Metric Vision, Institute for Automation, University of Leoben, Austria, www.metricvision.org, 2005.

[61] W. H. Foy, "Position-location solution by Taylor-series estimation," IEEE Trans. Aerosp. Electron. Syst., vol. AES-12, pp. 187 - 194, Mar. 1976.

[62] L. Yang and K.C. Ho, "An approximately efficient TDOA localization algorithm in closed-form for locating multiple disjoint sources with erroneous sensor positions," IEEE Trans. Signal Process., vol. 57, pp. 4598 - 4615, Dec. 2009.

[63] H.W. Sorenson, Parameter Estimation: Principles and Problems. New York: Marcel Dekker, 1980. 
[64] J. Zheng and Y. C. Wu, "Joint time synchronization and localization of an unknown node in wireless sensor networks," IEEE Trans. Signal Process., vol. 58, pp. 1309-1320, Mar. 2010.

[65] Y. T. Chan and K. C. Ho, "A simple and efficient estimator for hyperbolic location," IEEE Trans. Signal Process., vol. 42, pp. 1905-1915, Aug. 1994.

[66] R. J. Barton and D. Rao, "Performance capabilities of long-range UWB-IR TDOA localization systems," EURASIP J. Advances Signal Process., vol. 2008, 2008.

[67] S. Coraluppi, "Multistatic sonar localization," IEEE J. Ocean. Eng., vol. 31, pp. 964-974, Oct. 2006.

[68] G. Shirazi, M. Shenouda, and L. Lampe, "Second order cone programming for sensor network localization with anchor position uncertainty," in Proc. Workshop on Positioning Navigation and Communication (WPNC), Apr. 2011, pp. 51-55.

[69] R. Bhatia, Positive Definite Matrices. Princeton Series in Applied Mathematics, 2007. 